\title{
Protocol for Stereoselective Construction of Highly Functionalized Dienyl Sulfonyl Fluoride Warheads
}

Zai-Wei Zhang ${ }^{\mathrm{a}}$, Shi-Meng Wang ${ }^{\mathrm{b}}$, Wan-Yin Fang ${ }^{\mathrm{a}}$, Ravindar Lekkala ${ }^{\mathrm{a}}$, and Hua-Li Qin ${ }^{\mathrm{a}, *}$

a School of Chemistry, Chemical Engineering and Life Science, Wuhan University of Technology, 205 Luoshi Road, Wuhan, 430070, China

b School of Life Science, Wuchang University of Technology, Wuhan, 430223, P.R. China

*E-mail: qinhuali@whut.edu.cn.

\section{Table of content}

$\begin{array}{ll}\text { 1. Optimization of the reaction conditions. } & \mathrm{S} 2\end{array}$

2. NMR spectra $\quad$ S6

$\begin{array}{lr}\text { 3. Data of Crystal Structure of } \mathbf{3 f a} & \text { S199 }\end{array}$ 


\section{Optimization of the reaction conditions.}

Table S1. Screening of the catalysts ${ }^{\mathrm{a}}$.

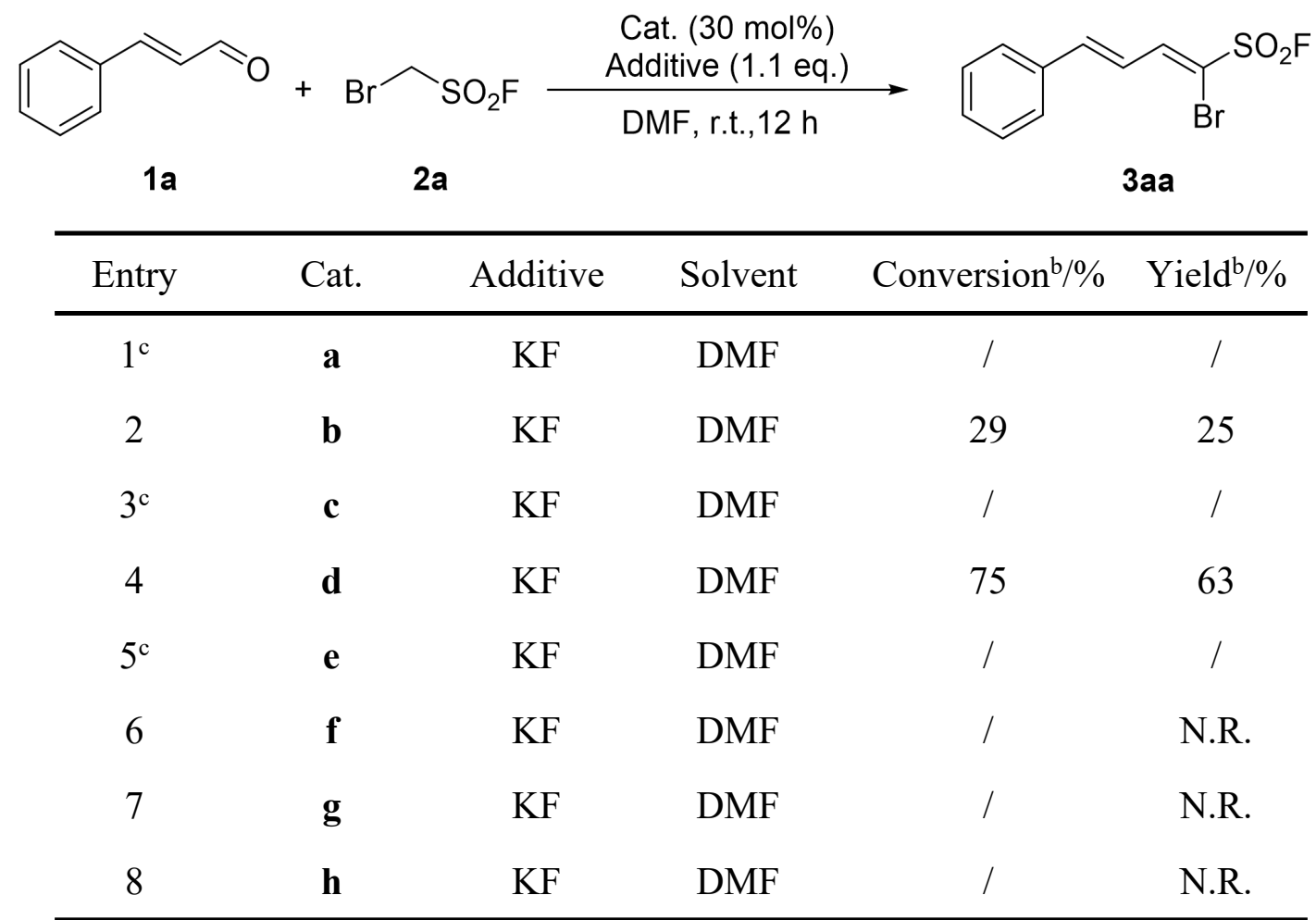

${ }^{\text {a }}$ Reaction conditions: cinnamaldehyde (1a, $\left.0.5 \mathrm{mmol}\right)$, additive (1.1 eq.) and catalyst (30 mol\%) were dissolved in DMF $(0.2 \mathrm{M}, 2.5 \mathrm{~mL})$ and the mixture was allowed to stir for 5 minutes before the subsequent addition of $\mathbf{2 a}$ (3.0 eq.). Then the stirring was lasted for $12 \mathrm{~h}$ at room temperature.

b The yield and conversion were determined by HPLC using 1a, 3aa as the external standards respectively $\left(t_{1 \mathrm{a}}=2.4 \mathrm{~min}, \lambda_{\max }=284.4 \mathrm{~nm} ; t_{3 \mathrm{aa}}=4.1 \mathrm{~min}, \lambda_{\max }=322.5 \mathrm{~nm}\right.$; acetonitrile $/$ water $=80: 20(\mathrm{v} / \mathrm{v}))$.

${ }^{\mathrm{c}}$ a mass of mixture was detected by TLC.

N.R. $=$ no reaction<smiles>C1COCCN1</smiles><smiles>C1CCNCC1</smiles>

a<smiles>CN1CCNCC1</smiles>

c<smiles>C1CCNC1</smiles>

d<smiles>O=C(O)C1CCCN1</smiles>

e<smiles>Nc1ccccc1</smiles><smiles>CC(C)(C)N</smiles><smiles>NC1CCCCC1</smiles>

h 
Table S2. Screening of the additive and solvent ${ }^{\mathrm{a}}$.

\begin{tabular}{|c|c|c|c|c|c|}
\hline $1 a$ & 2 & & & & 3aa \\
\hline Entry & Cat. & Additive & Solvent & Conversion $\mathrm{b} / \%$ & Yield $/ \%$ \\
\hline 1 & pyrrolidine & $\mathrm{KF}$ & $\mathrm{DMF}$ & 75 & 63 \\
\hline 2 & pyrrolidine & $\mathrm{KF}$ & DCM & 97 & 69 \\
\hline 3 & pyrrolidine & $\mathrm{KF}$ & THF & 93 & 88 \\
\hline 4 & pyrrolidine & $\mathrm{KF}$ & Dioxane & 75 & 64 \\
\hline 5 & pyrrolidine & $\mathrm{KF}$ & $\mathrm{CHCl}_{3}$ & 97 & 62 \\
\hline 6 & pyrrolidine & $\mathrm{KF}$ & Toluene & 54 & 29 \\
\hline 7 & pyrrolidine & $\mathrm{KF}$ & $\mathrm{DCE}$ & 94 & 60 \\
\hline 8 & pyrrolidine & $\mathrm{KF}$ & DMSO & 89 & 68 \\
\hline 9 & pyrrolidine & $\mathrm{KF}$ & Acetonitrile & 81 & 62 \\
\hline 10 & pyrrolidine & I & THF & 93 & 89 \\
\hline 11 & pyrrolidine & I & dry THF & 93 & 87 \\
\hline
\end{tabular}

a Reaction conditions: cinnamaldehyde (1a, $0.5 \mathrm{mmol})$, additive (1.1 eq.) and pyrrolidine $(30 \mathrm{~mol} \%)$ were dissolved in solvent $(0.2 \mathrm{M}, 2.5 \mathrm{~mL})$ and the mixture was allowed to stir for 5 minutes before the subsequent addition of $2 \mathbf{a}$ (3.0 eq.). Then the stirring was lasted for $12 \mathrm{~h}$ at room temperature.

$\mathrm{b}$ The yield and conversion were determined by HPLC using 1a, 3aa as the external standards respectively $\left(t_{1 \mathrm{a}}=2.4 \mathrm{~min}, \lambda_{\max }=284.4 \mathrm{~nm} ; t_{3 \mathrm{aa}}=4.1 \mathrm{~min}, \lambda_{\max }=322.5 \mathrm{~nm}\right.$; acetonitrile $/$ water $=80: 20(\mathrm{v} / \mathrm{v}))$. 
Table S3. Screening of the catalyst loadinga

\begin{tabular}{|c|c|c|c|}
\hline $1 a$ & $2 a$ & & 3aa \\
\hline Entry & $\mathbf{X}(\mathrm{mol} \%)$ & Conversion $\mathrm{b} / \%$ & Yield $/ \%$ \\
\hline 1 & 30 & 93 & 89 \\
\hline 2 & 50 & 99 & 94 \\
\hline 3 & 60 & 100 & 95 \\
\hline 3 & 70 & 100 & 94 \\
\hline 4 & 100 & 100 & 88 \\
\hline 5 & 120 & 100 & 87 \\
\hline 6 & 150 & 100 & 83 \\
\hline
\end{tabular}

${ }^{a}$ Reaction conditions: cinnamaldehyde (1a, $\left.0.5 \mathrm{mmol}\right)$ and pyrrolidine (X mol\%) were dissolved in THF $(0.2 \mathrm{M}, 2.5 \mathrm{~mL})$ and the mixture was allowed to stir for 5 minutes before the subsequent addition of $\mathbf{2 a}$ (3.0 eq.). Then the stirring was lasted for 20 minutes at room temperature.

${ }^{\mathrm{b}}$ The yield and conversion were determined by HPLC using 1a, 3aa as the external standards respectively $\left(t_{1 \mathrm{a}}=2.4 \mathrm{~min}, \lambda_{\max }=284.4 \mathrm{~nm} ; t_{3 \mathrm{aa}}=4.1 \mathrm{~min}, \lambda_{\max }=322.5 \mathrm{~nm}\right.$; acetonitrile $/$ water $=80: 20(\mathrm{v} / \mathrm{v}))$. 
Table S4. Screening of the bromomethanesulfonyl fluoride loading ${ }^{\mathrm{a}}$.

\begin{tabular}{|c|c|c|c|}
\hline $1 a$ & $2 a$ & & 3aa \\
\hline Entry & $\mathbf{2 a}$ (X equiv.) & Conversion $/ \%$ & Yield $^{\mathrm{b}} \%$ \\
\hline 1 & 1.2 & 93 & 74 \\
\hline 2 & 1.5 & 100 & 78 \\
\hline 3 & 2.0 & 100 & 82 \\
\hline 4 & 3.0 & 100 & 95 \\
\hline 5 & 3.5 & 100 & 95 \\
\hline
\end{tabular}

a Reaction conditions: cinnamaldehyde (1a, $0.5 \mathrm{mmol})$ and pyrrolidine (60 mol $\%)$ were dissolved in THF $(0.2 \mathrm{M}, 2.5 \mathrm{~mL})$ and the mixture was allowed to stir for 5 minutes before the subsequent addition of 2a (X eq.). Then the stirring was lasted for 20 minutes at room temperature.

$\mathrm{b}$ The yield and conversion were determined by HPLC using 1a, 3aa as the external standards respectively $\left(t_{1 \mathrm{a}}=2.4 \mathrm{~min}, \lambda_{\max }=284.4 \mathrm{~nm} ; t_{3 \mathbf{a a}}=4.1 \mathrm{~min}, \lambda_{\max }=322.5 \mathrm{~nm}\right.$; acetonitrile $/$ water $=80: 20(\mathrm{v} / \mathrm{v}))$.

Table S5. Screening of the concentration ${ }^{\mathrm{a}}$.

\begin{tabular}{|c|c|c|c|}
\hline $1 a$ & $2 a$ & & 3 aa \\
\hline Entry & Concentration (X I & Conversion $/ \%$ & Yield $/ \%$ \\
\hline 1 & 0.125 & 100 & 93 \\
\hline 2 & 0.2 & 100 & 95 \\
\hline 3 & 0.25 & 100 & 94 \\
\hline 4 & 0.3 & 100 & 93 \\
\hline
\end{tabular}

a Reaction conditions: cinnamaldehyde (1a, $0.5 \mathrm{mmol})$ and pyrrolidine (60 mol $\%)$ were dissolved in THF (X M) and the mixture was allowed to stir for 5 minutes before the subsequent addition of $2 \mathbf{a}$ ( 3 eq.). Then the stirring was lasted for 20 minutes at room temperature.

b The yield and conversion were determined by HPLC using 1a, 3aa as the external standards respectively $\left(t_{1 \mathbf{a}}=2.4 \mathrm{~min}, \lambda_{\max }=284.4 \mathrm{~nm} ; t_{3 \mathbf{a a}}=4.1 \mathrm{~min}, \lambda_{\max }=322.5 \mathrm{~nm}\right.$; acetonitrile $/$ water $=80: 20(\mathrm{v} / \mathrm{v}))$. 
2.NMR spectra.

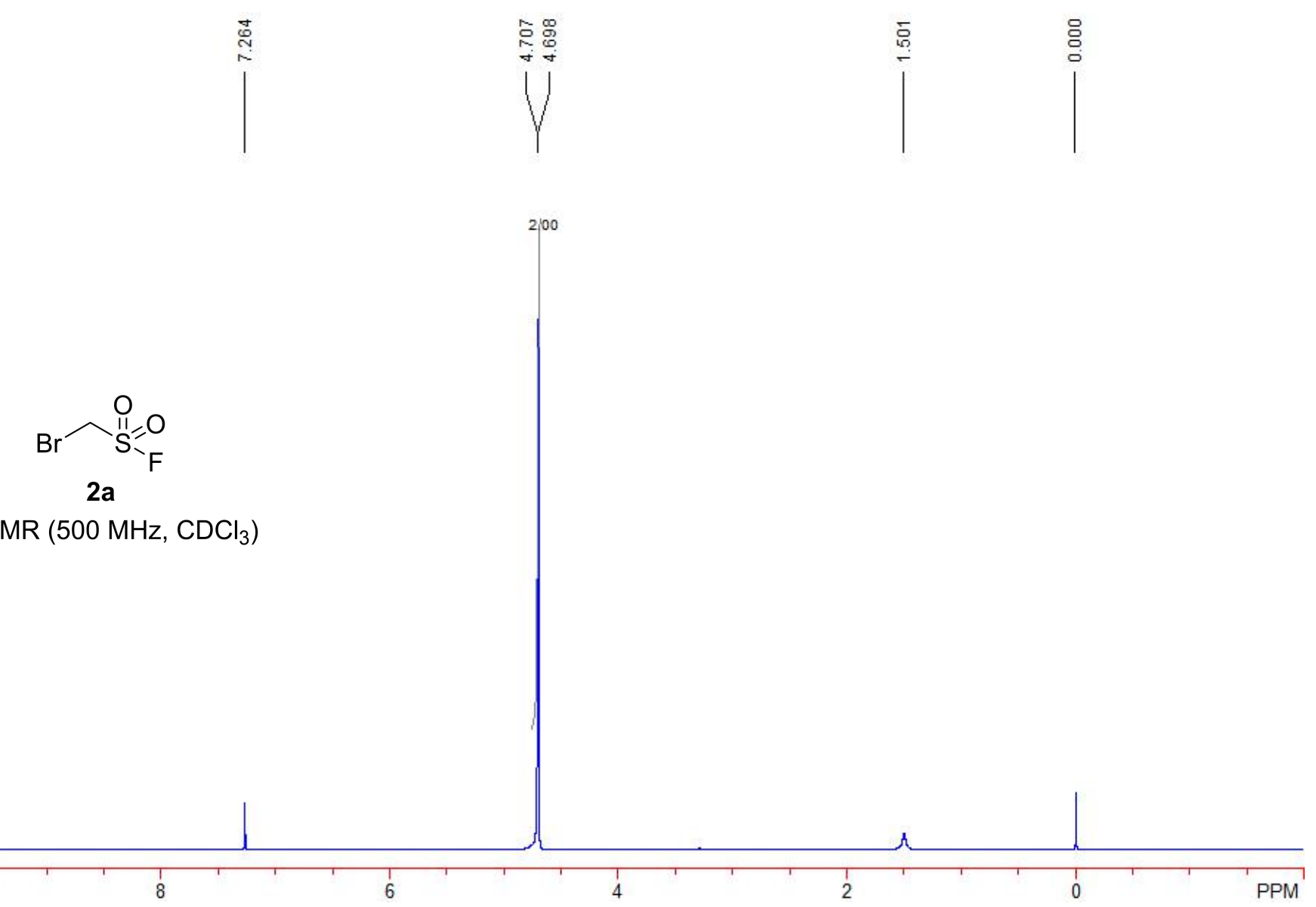


yj

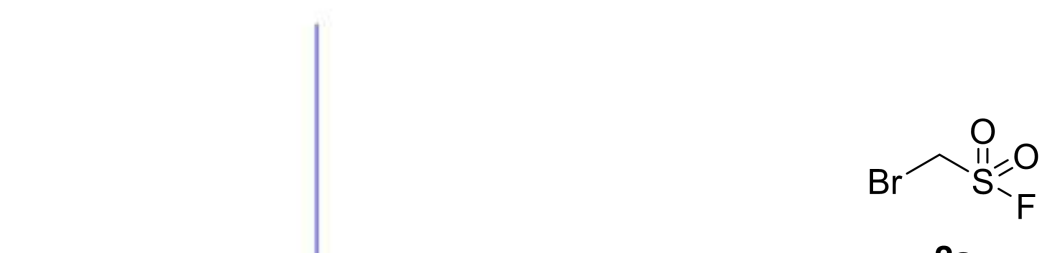

${ }^{19} \mathrm{~F} \mathrm{NMR}\left(471 \mathrm{MHz}, \mathrm{CDCl}_{3}\right)$ 


$$
\mathrm{Br} \sim \stackrel{\mathrm{O}}{\mathrm{I}}=\mathrm{O}
$$

$2 a$

${ }^{13} \mathrm{C}$ NMR (126 MHz, $\left.\mathrm{CDCl}_{3}\right)$

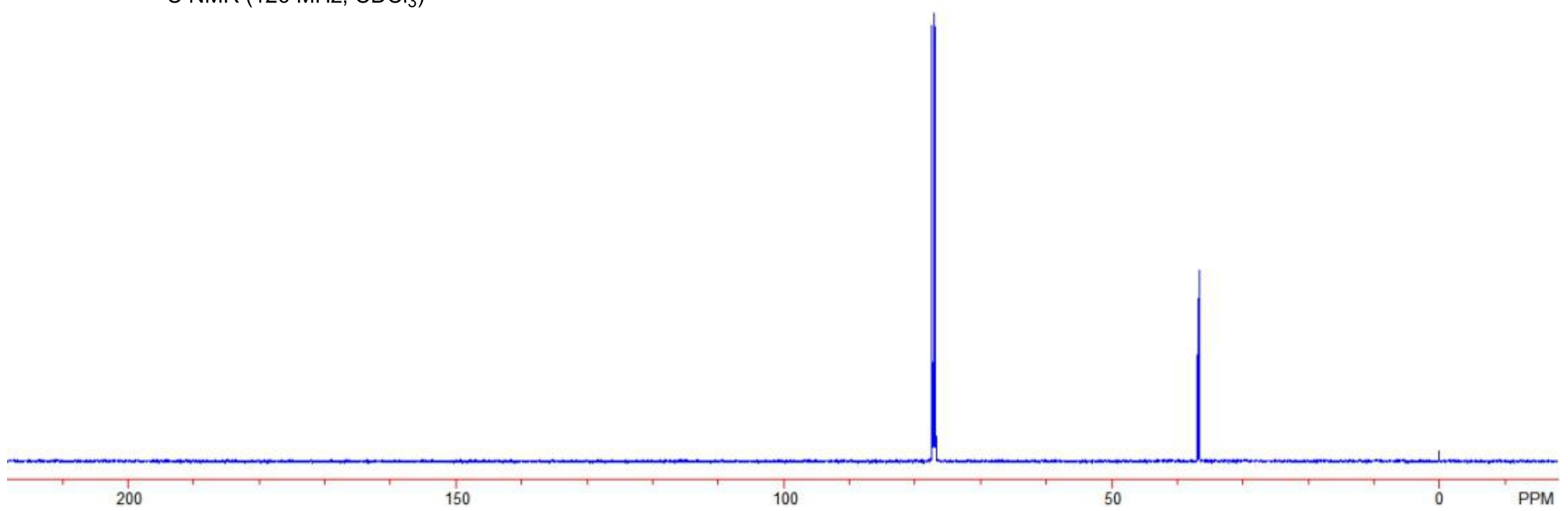




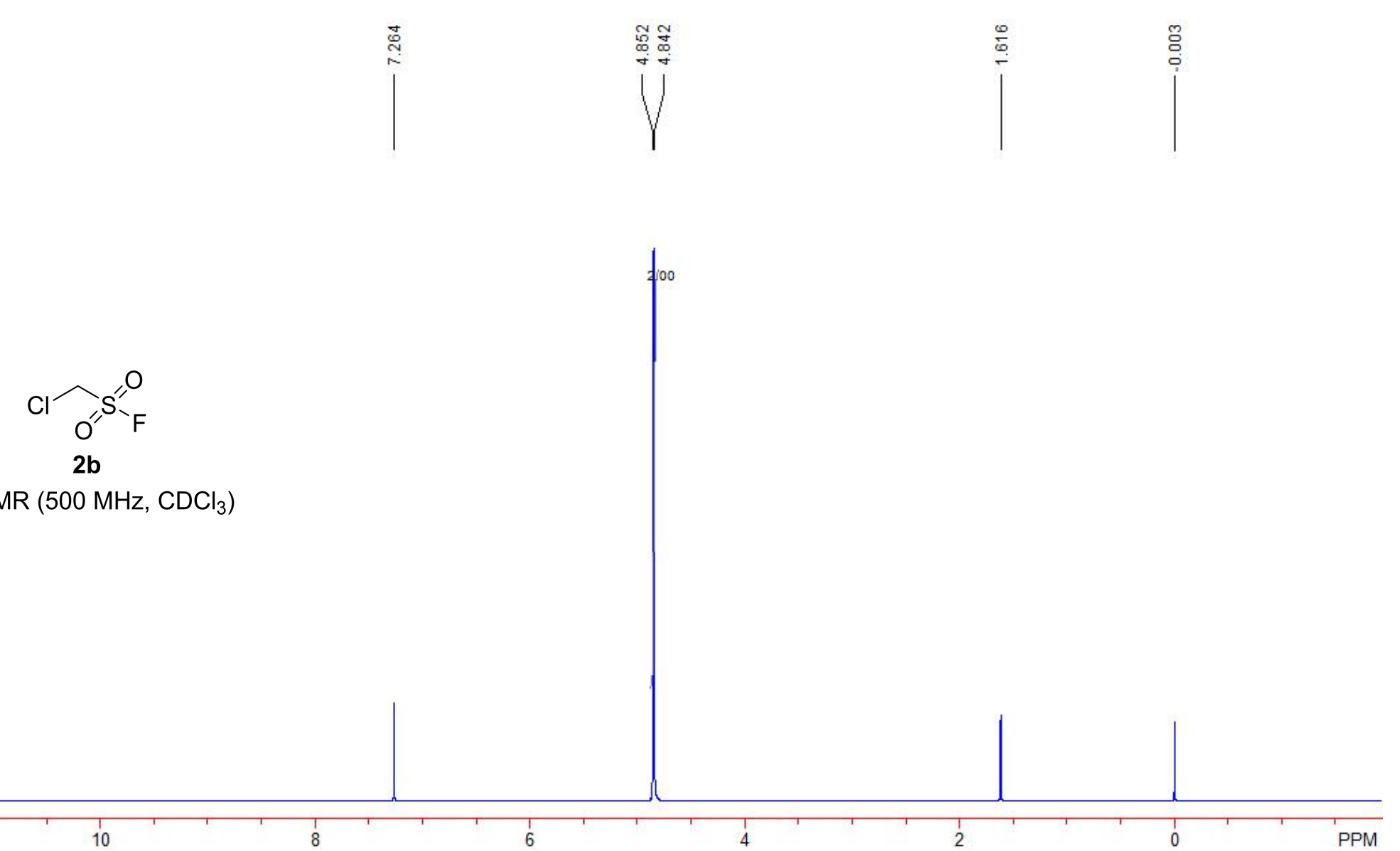




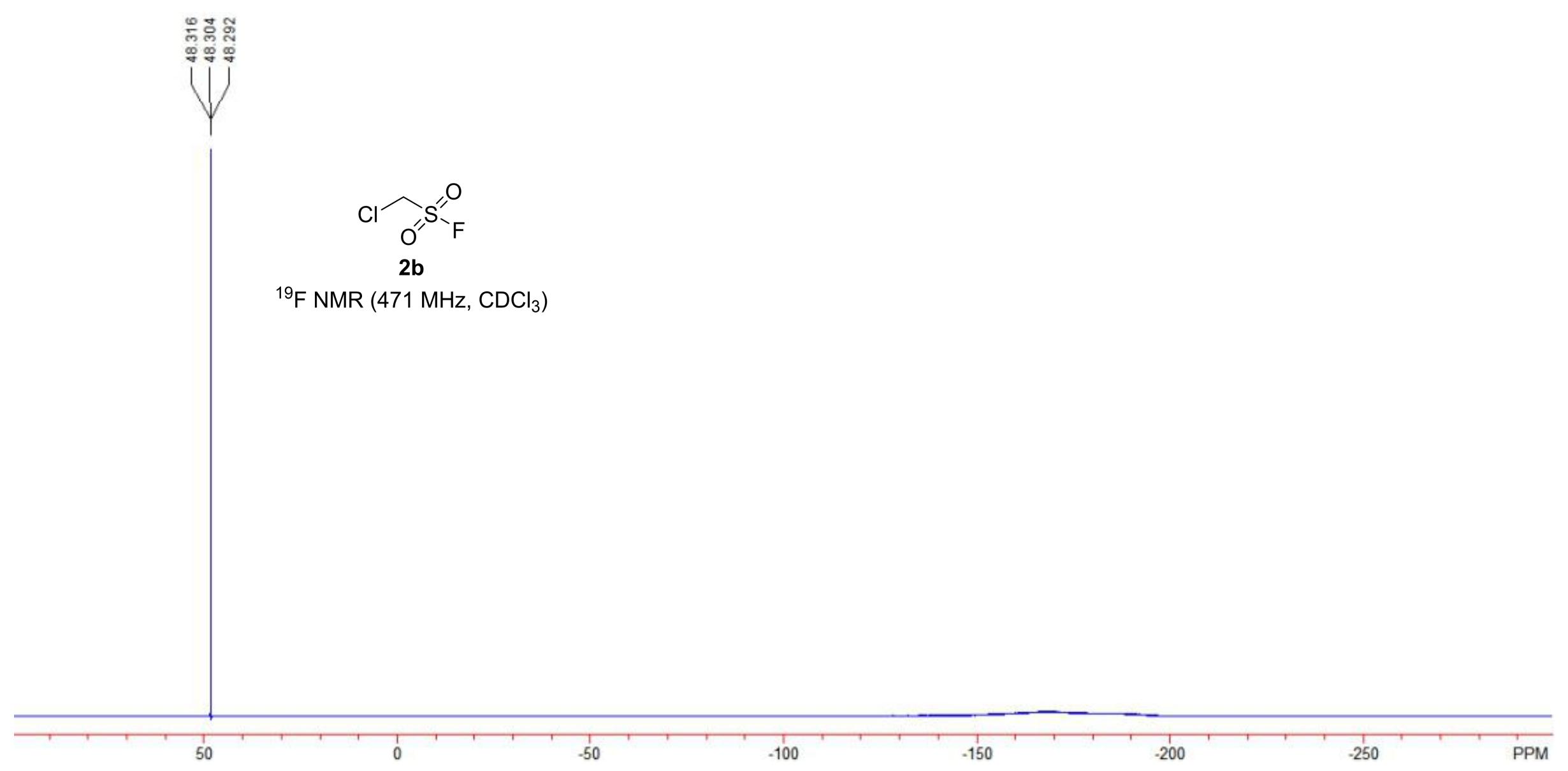




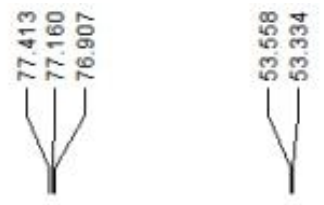

$$
\mathrm{Cl}_{\mathrm{O}^{\prime \prime}}^{\text {s" }} \text {. }
$$

${ }^{13} \mathrm{C}$ NMR $\left(126 \mathrm{MHz}, \mathrm{CDCl}_{3}\right.$ )

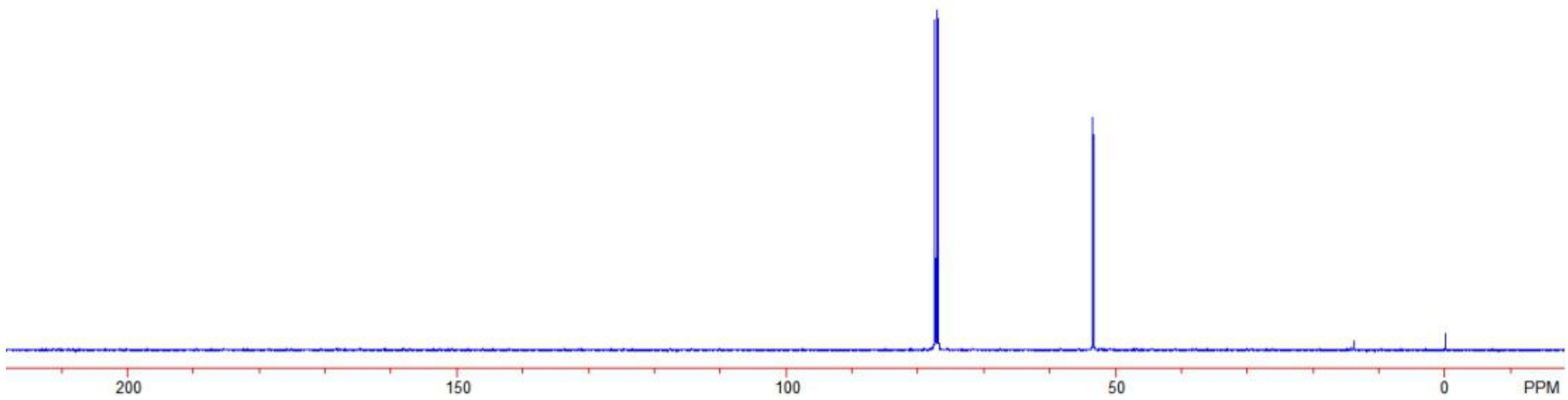




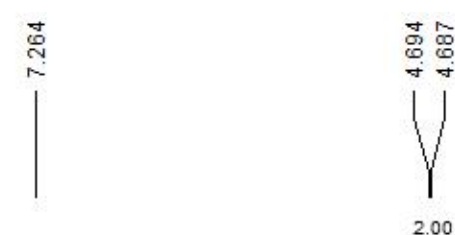

$$
{ }^{1} \mathrm{HNMR}\left(500 \mathrm{MHz}, \mathrm{CDCl}_{3}\right)
$$

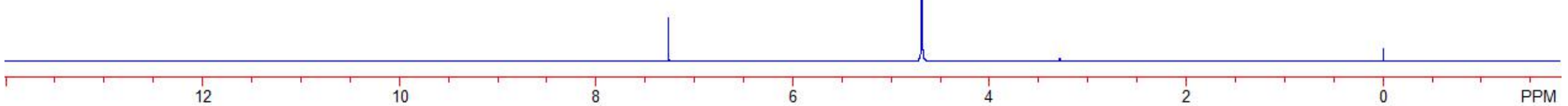


1

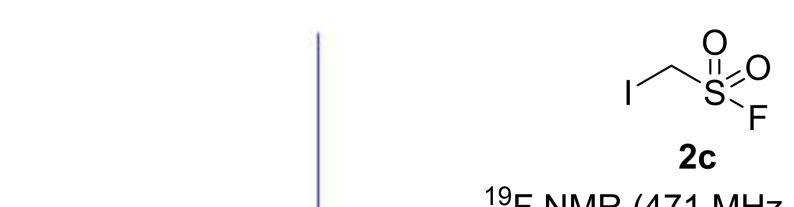

${ }^{19} \mathrm{~F} \mathrm{NMR}\left(471 \mathrm{MHz}, \mathrm{CDCl}_{3}\right)$ 


$$
\sim_{2 c}^{O 11}=0
$$

${ }^{13} \mathrm{C}$ NMR $\left(126 \mathrm{MHz}, \mathrm{CDCl}_{3}\right)$

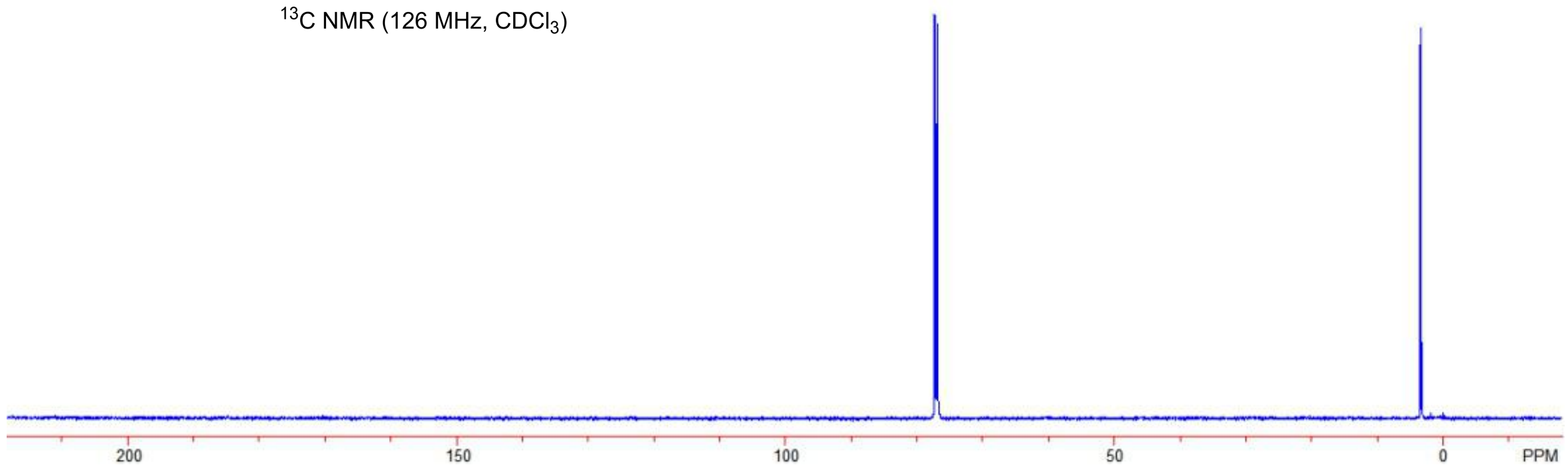



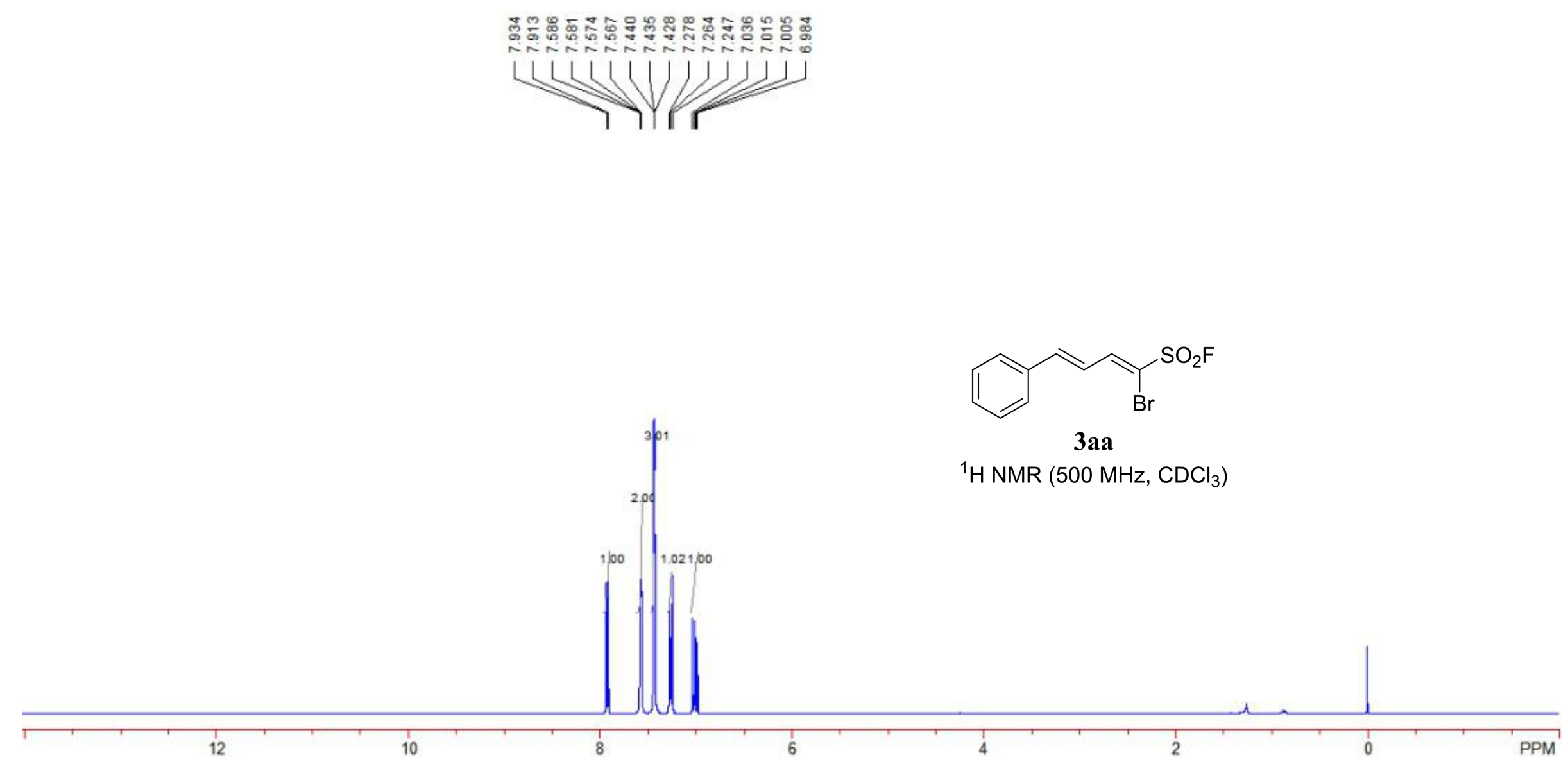


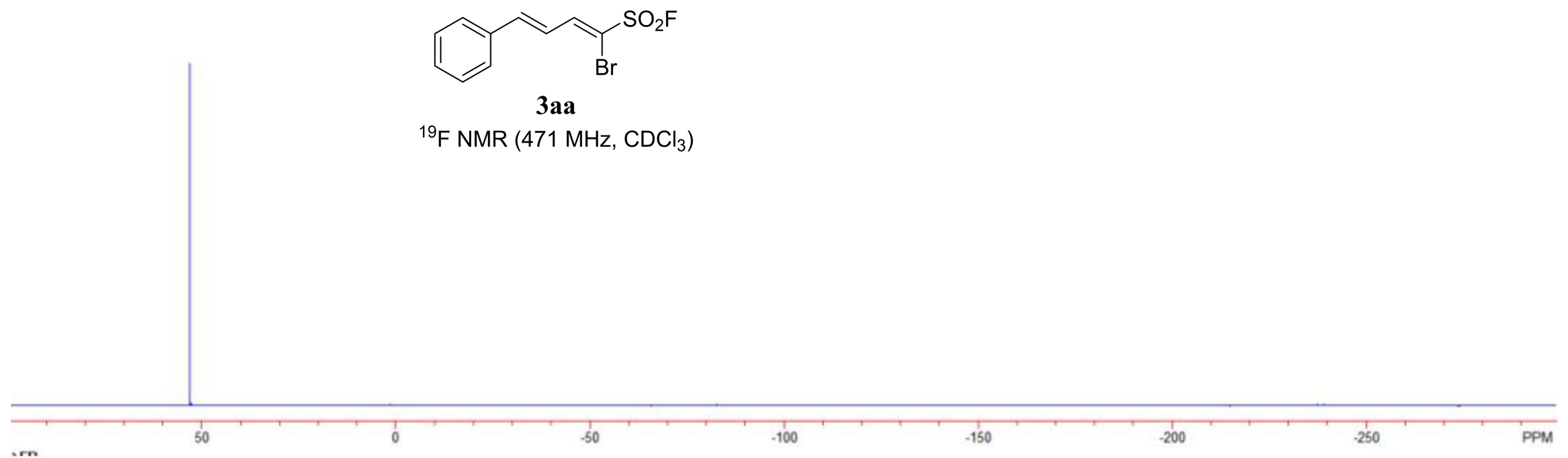




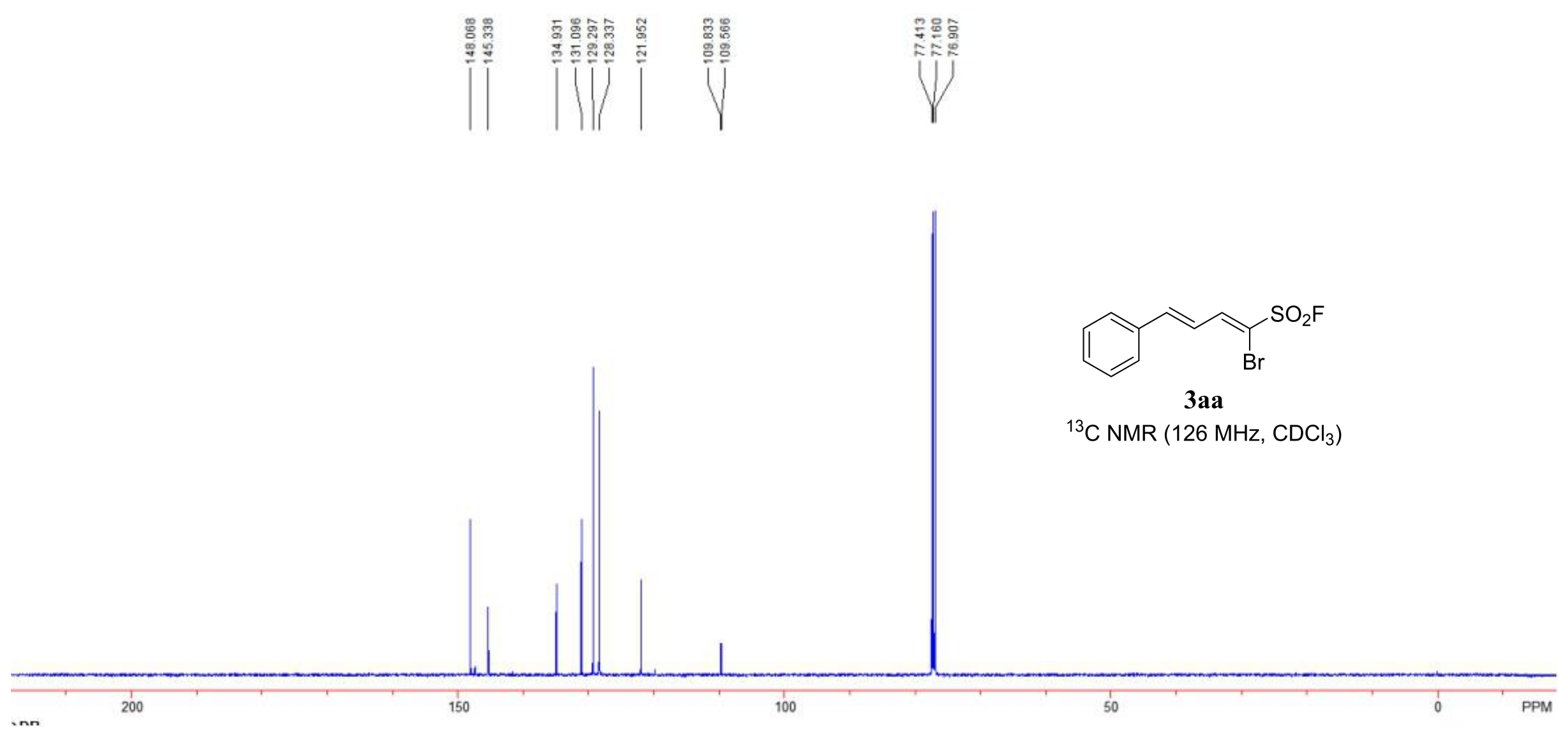




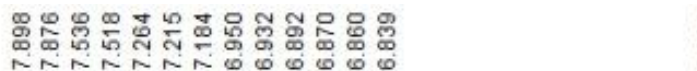

uMvini

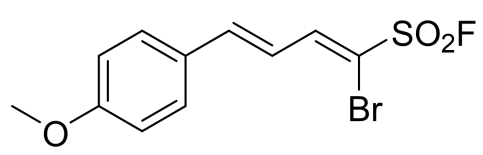

3 ba

${ }^{1} \mathrm{H} \mathrm{NMR}\left(500 \mathrm{MHz}, \mathrm{CDCl}_{3}\right)$

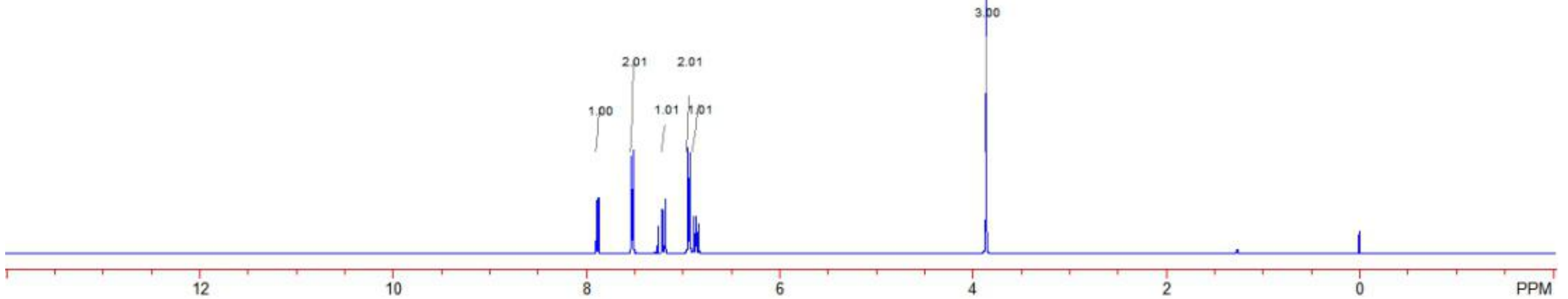




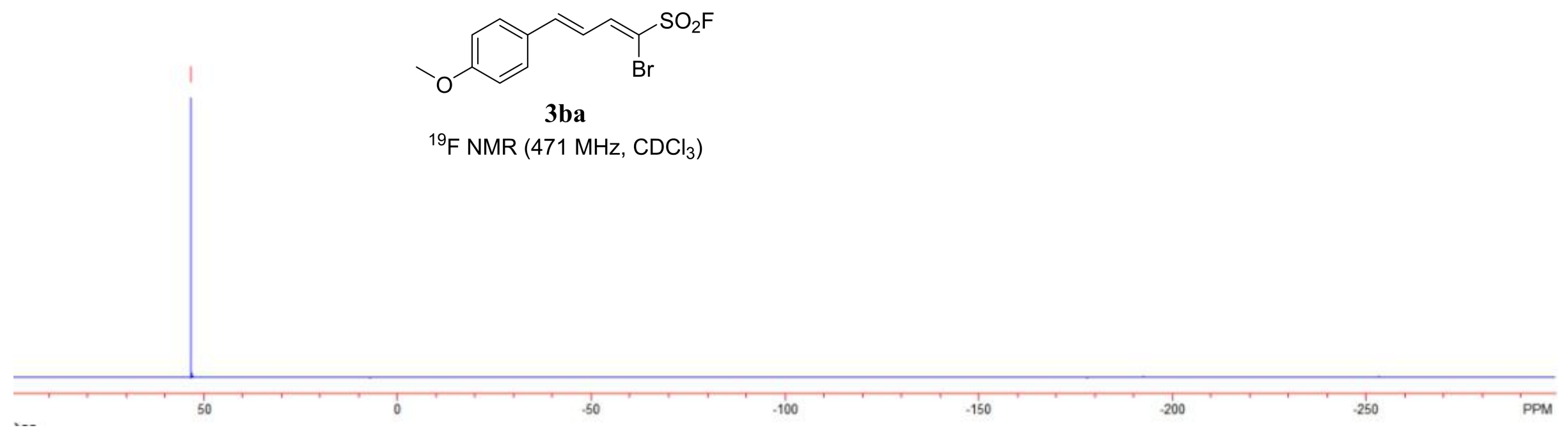



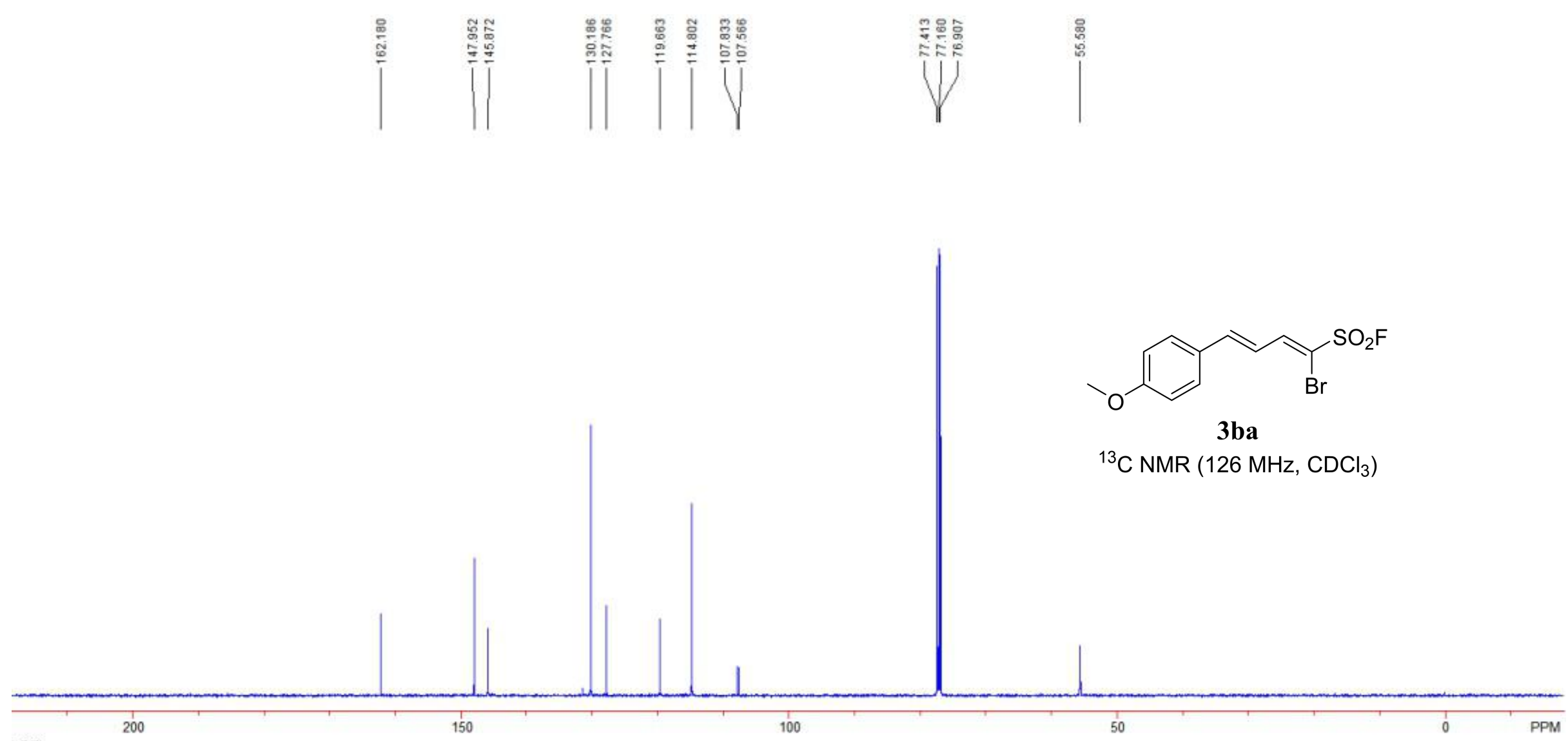


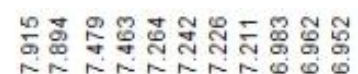

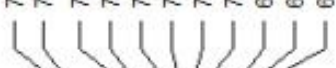

I I
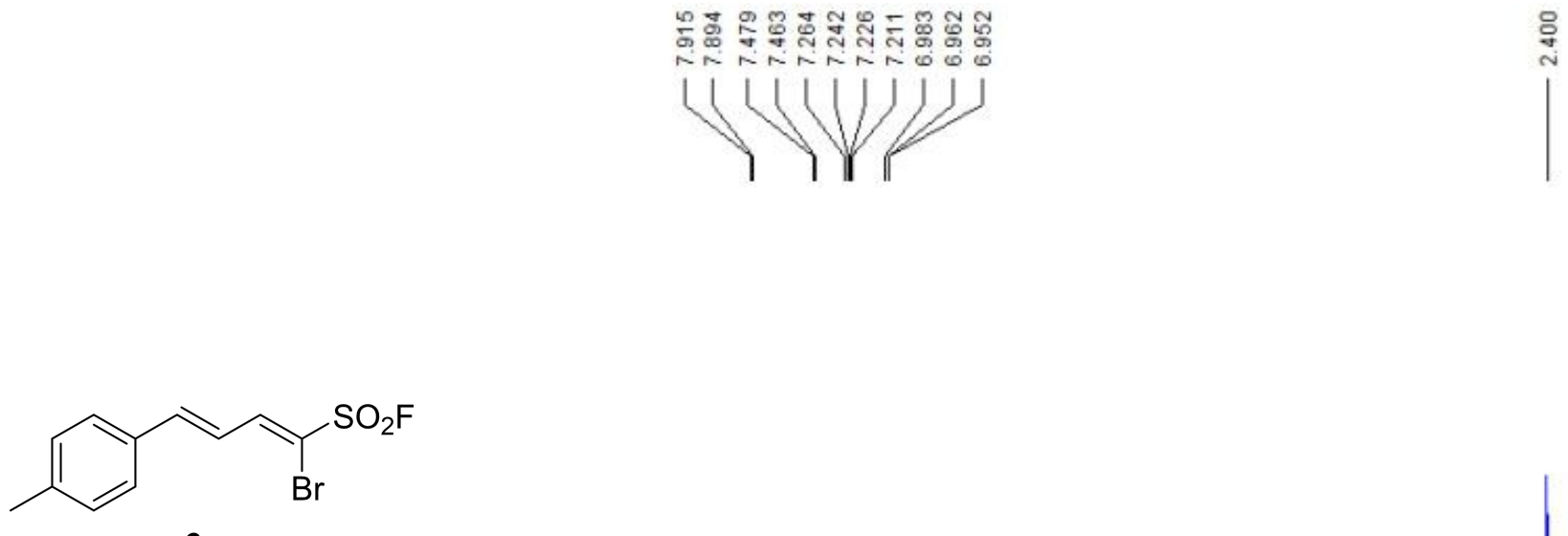

$3 \mathrm{ca}$

${ }^{1} \mathrm{H}$ NMR $\left(500 \mathrm{MHz}, \mathrm{CDCl}_{3}\right)$

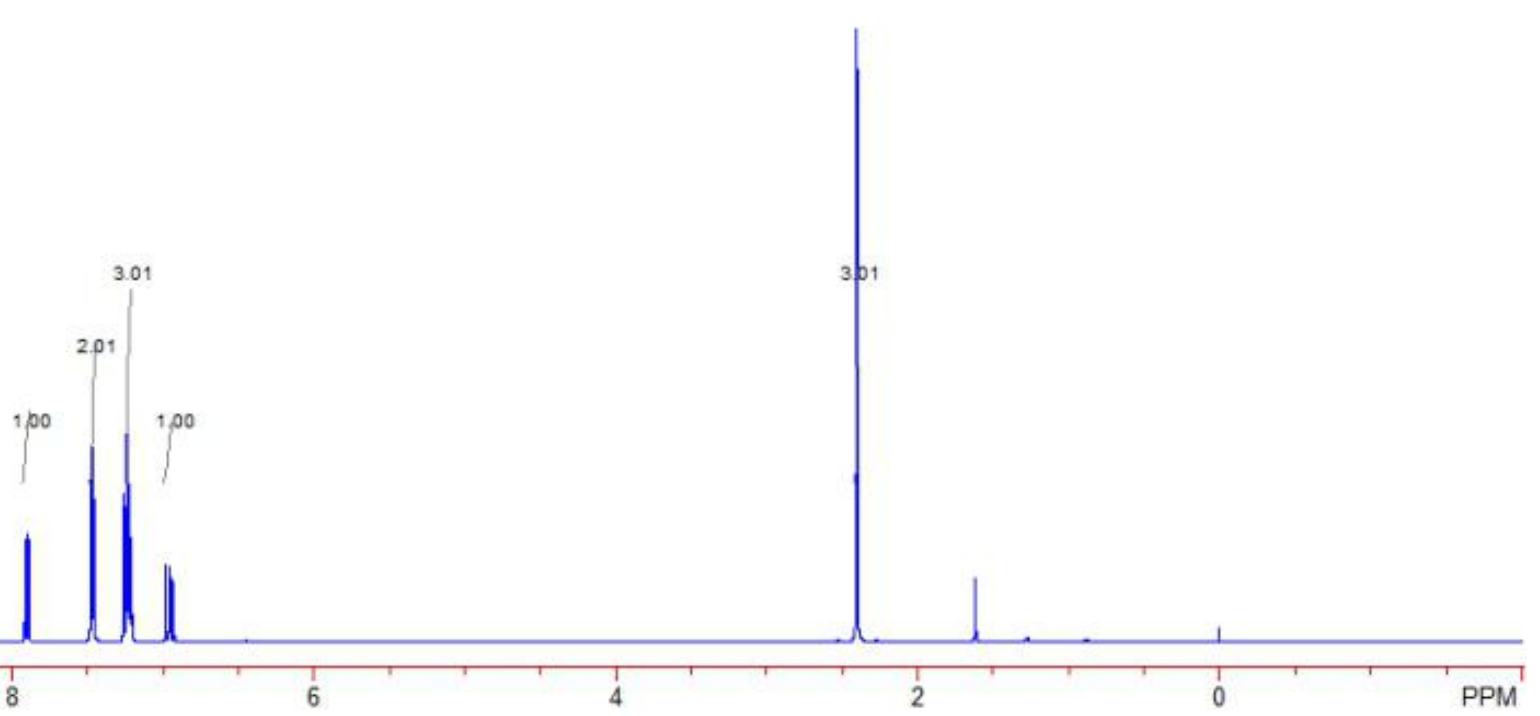




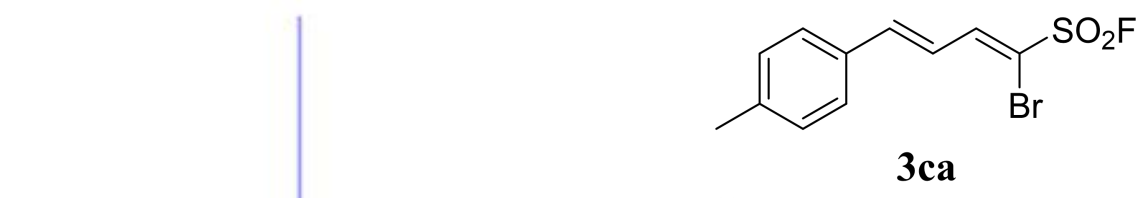

${ }^{19} \mathrm{~F}$ NMR (471 MHz, $\mathrm{CDCl}_{3}$ )

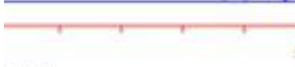

50

.50 

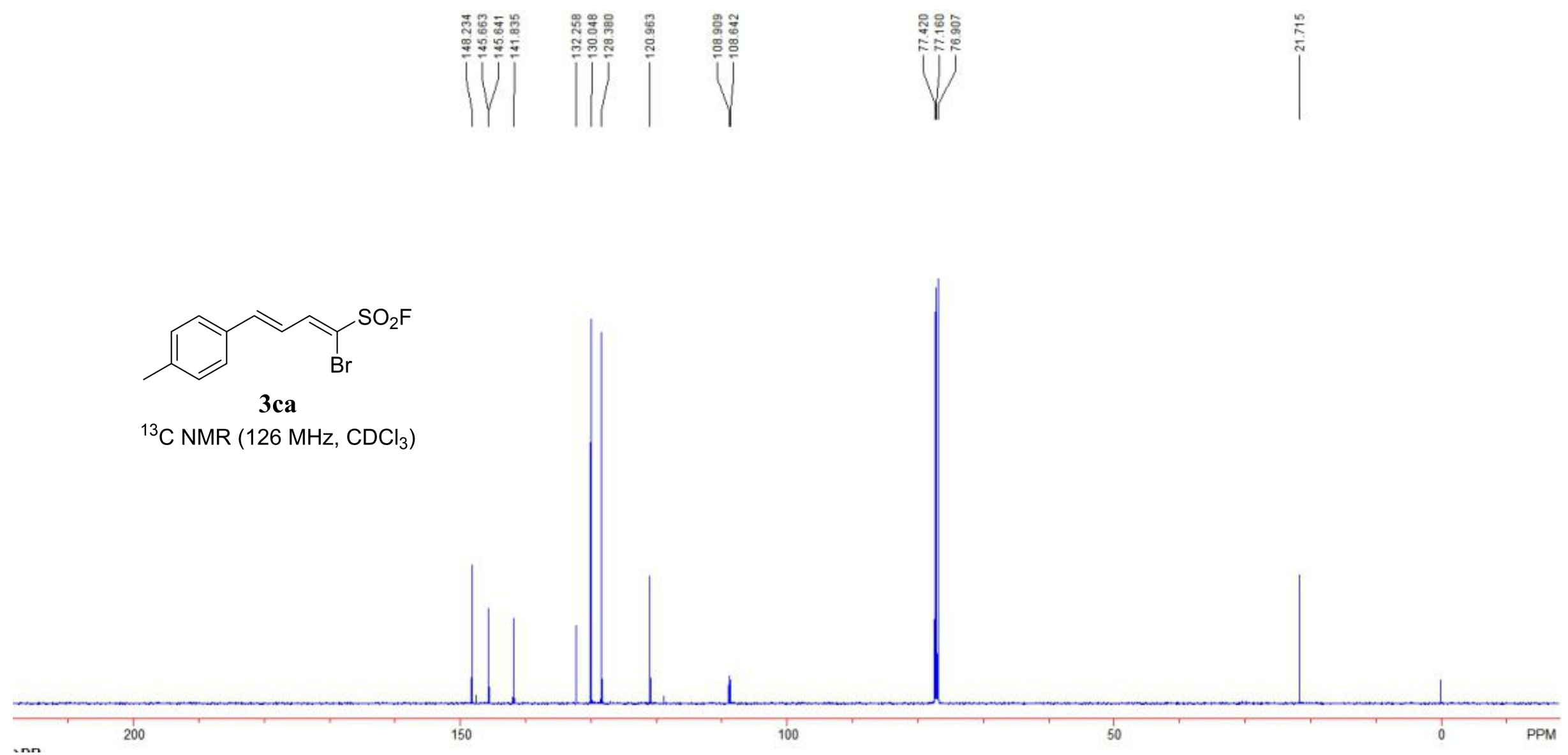


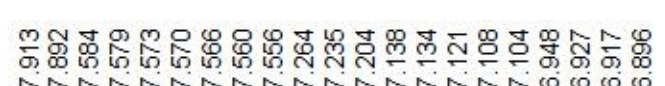

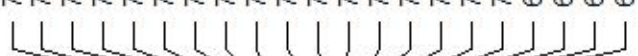

I $1 / 1$

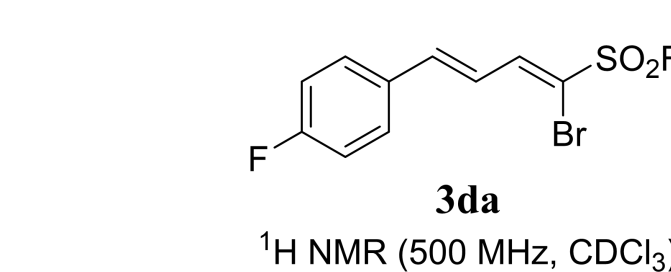

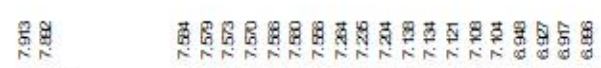

$\Rightarrow \quad 4$ का

${ }^{1} \mathrm{H} \mathrm{NMR}\left(500 \mathrm{MHz}, \mathrm{CDCl}_{3}\right)$
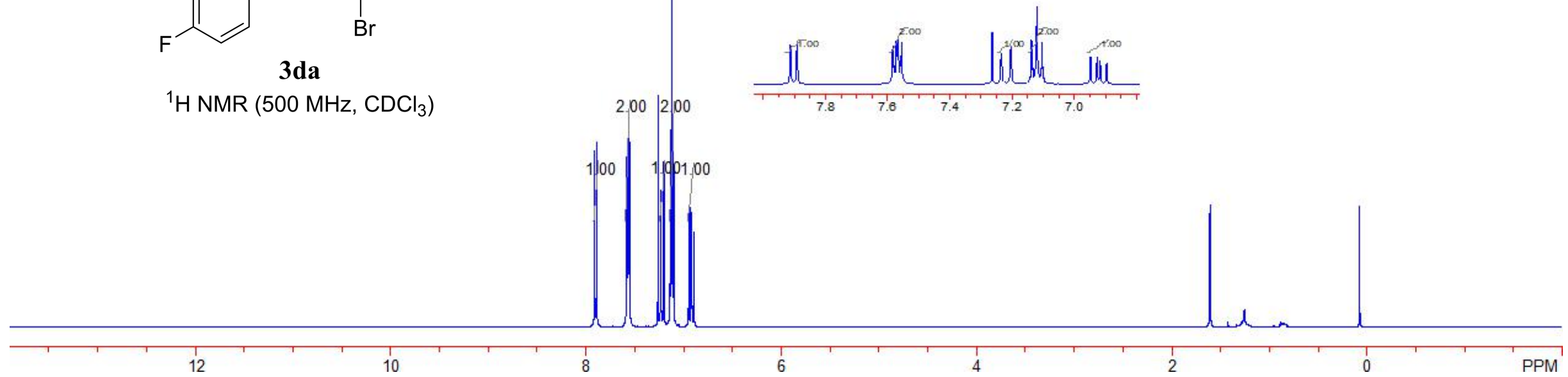
作

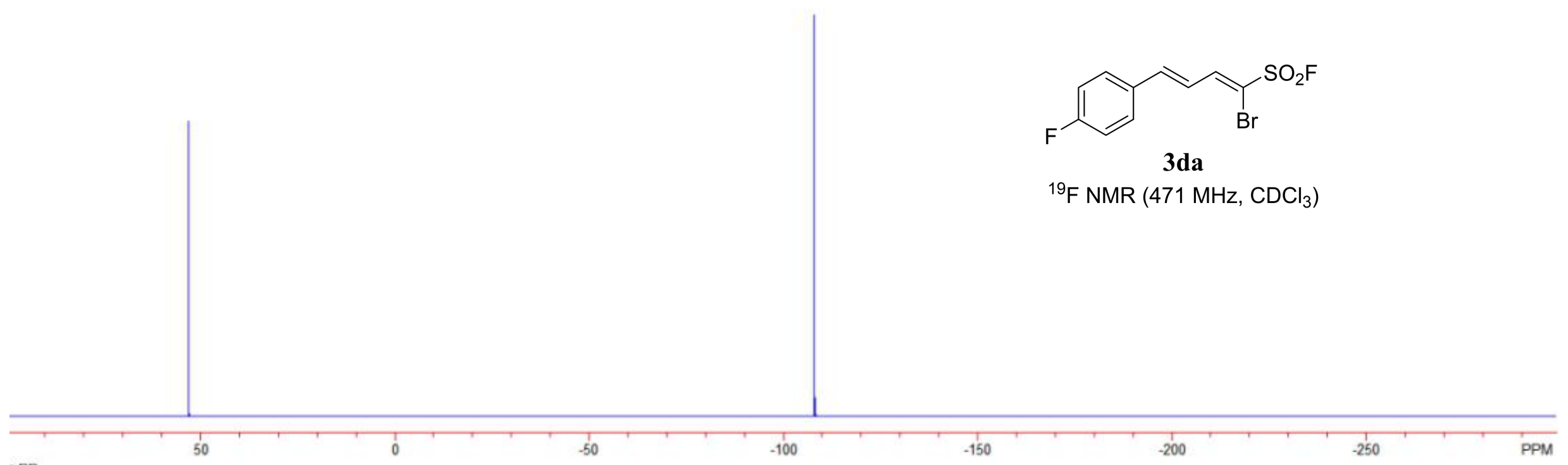




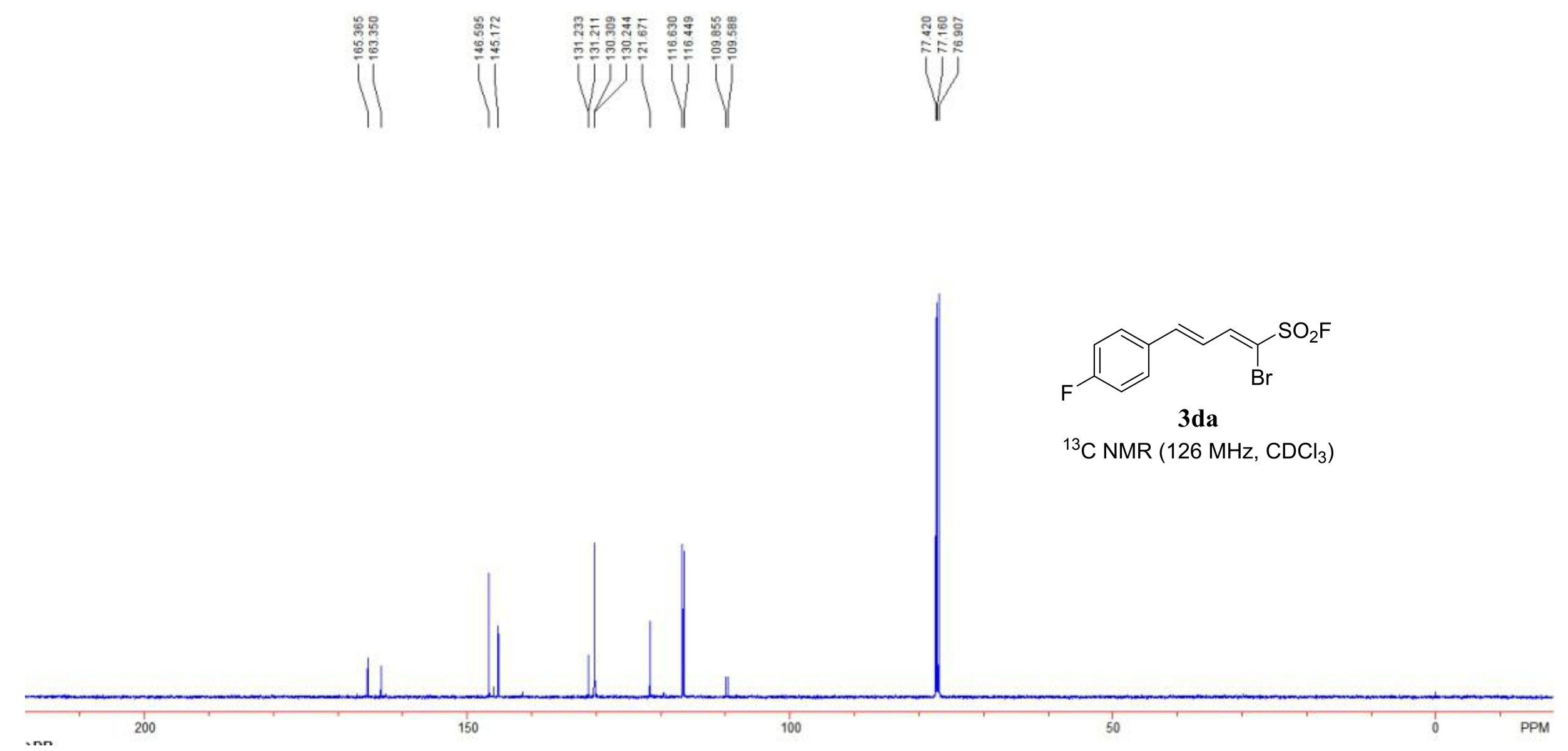



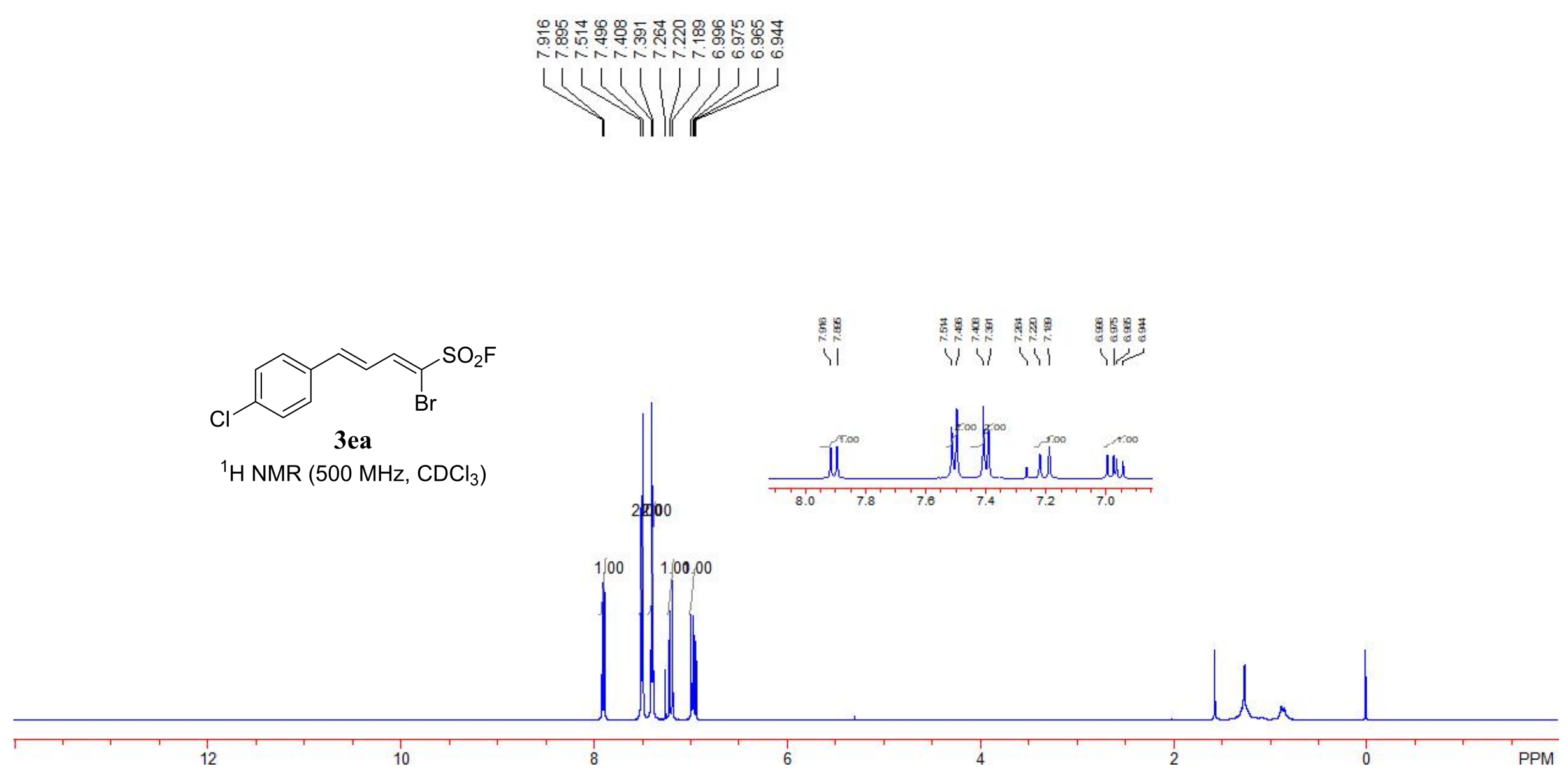


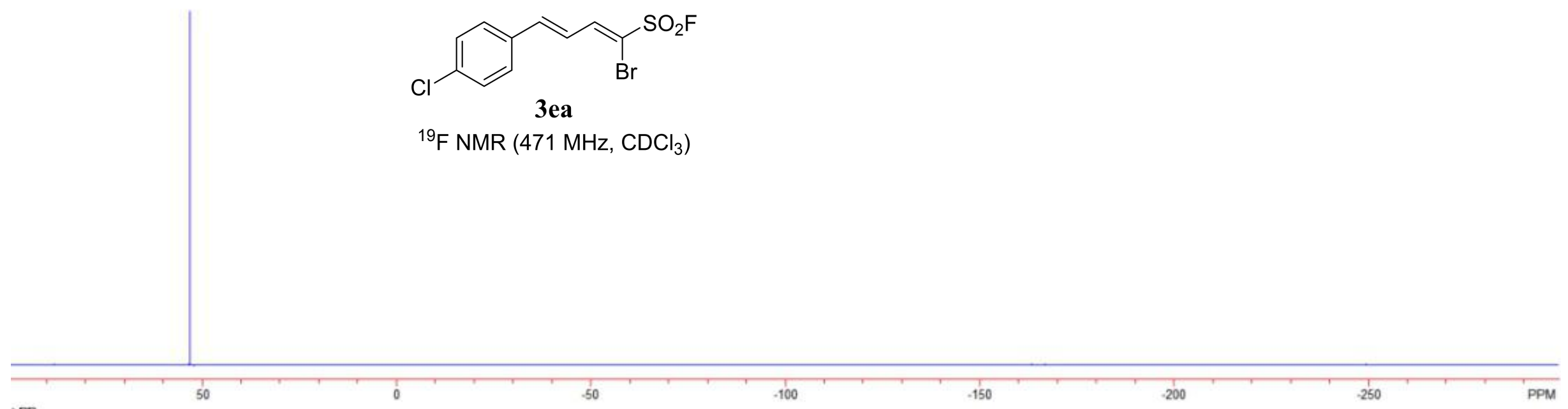



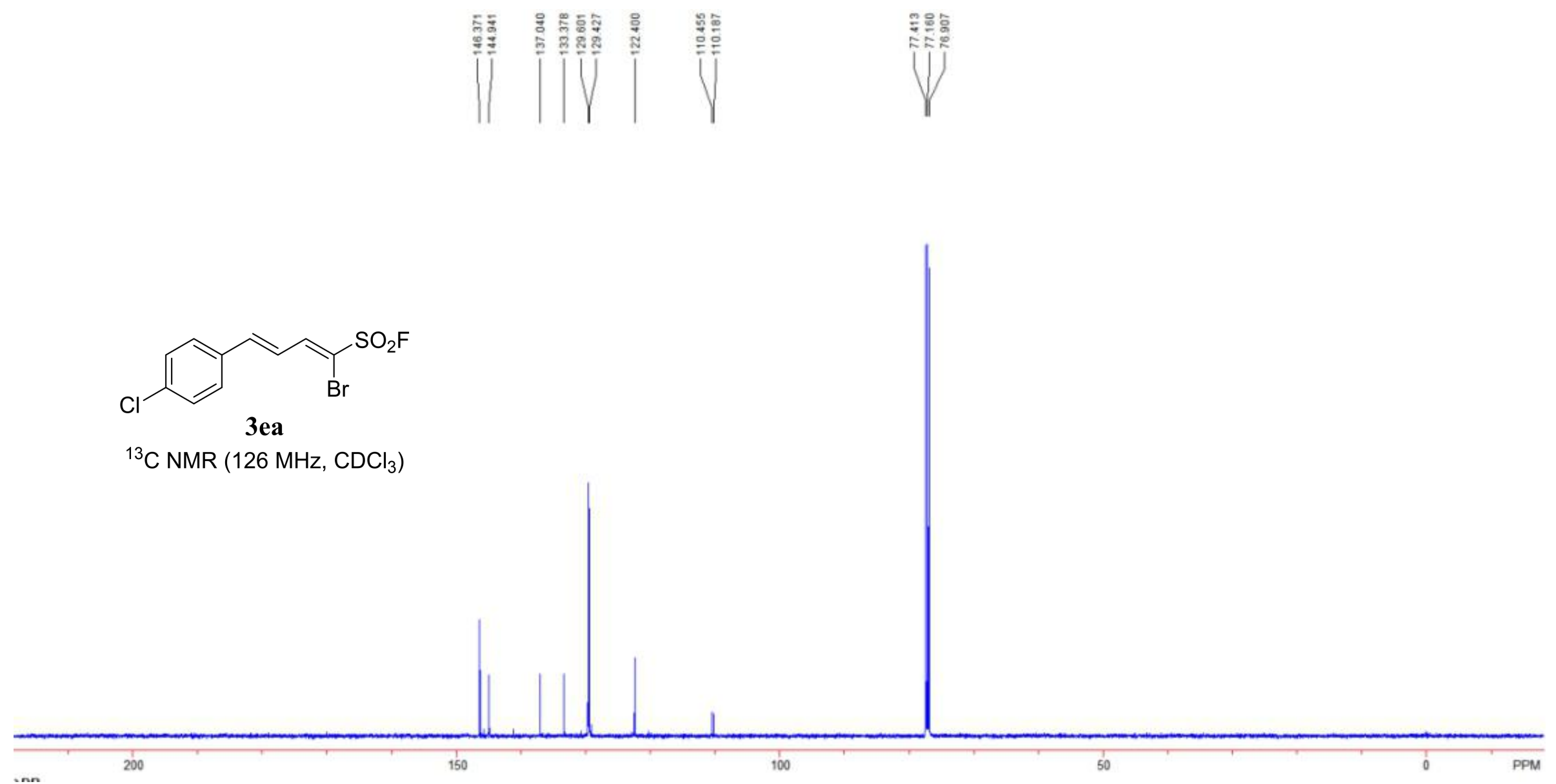


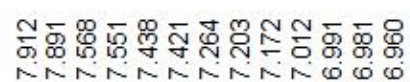

uMU

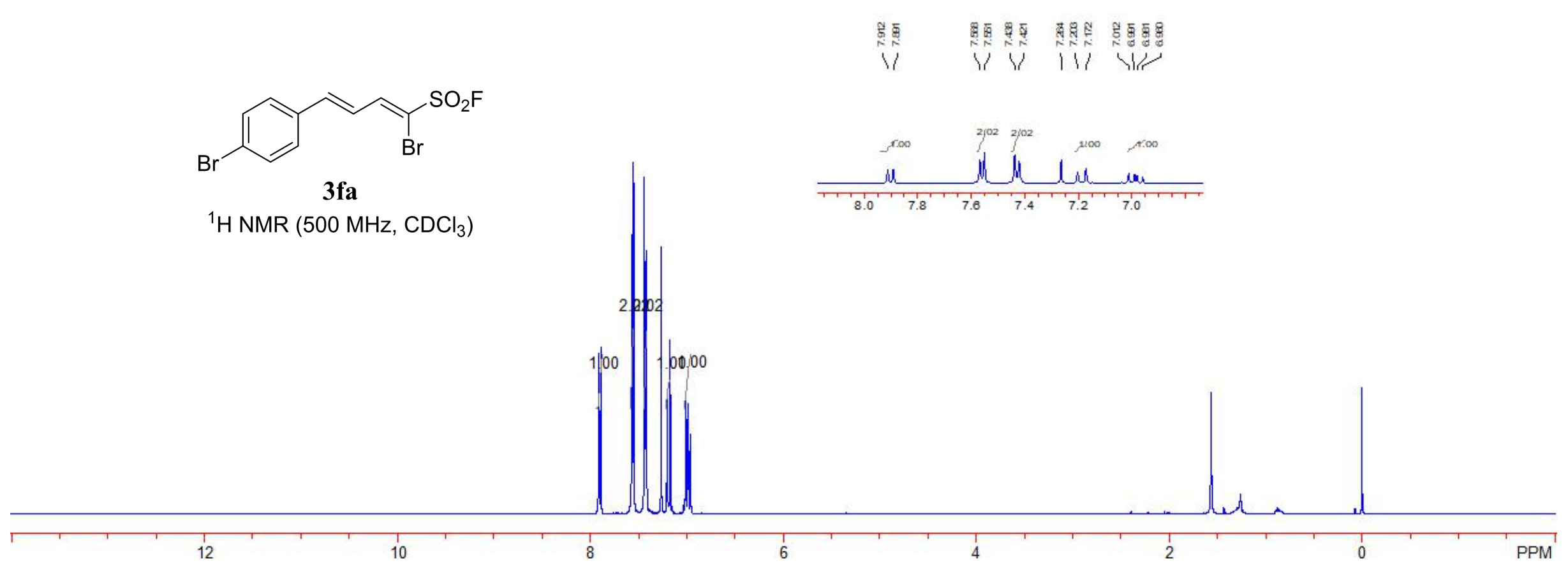




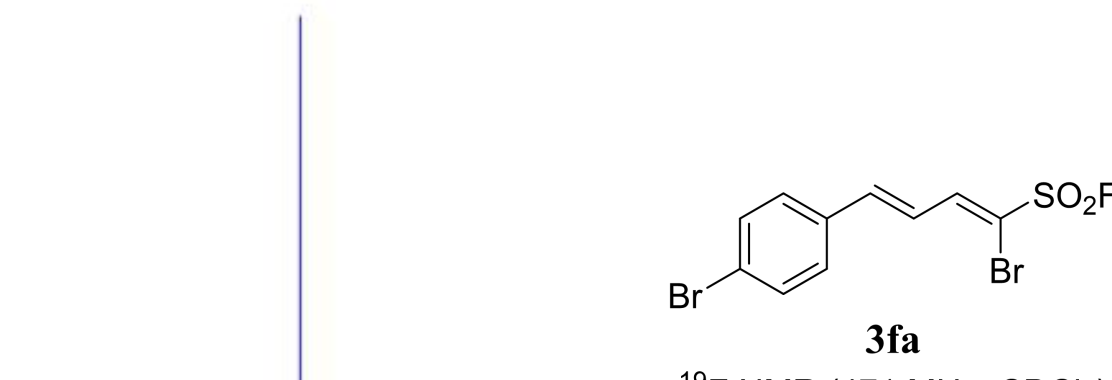

${ }^{19} \mathrm{~F} \mathrm{NMR}\left(471 \mathrm{MHz}, \mathrm{CDCl}_{3}\right)$ 


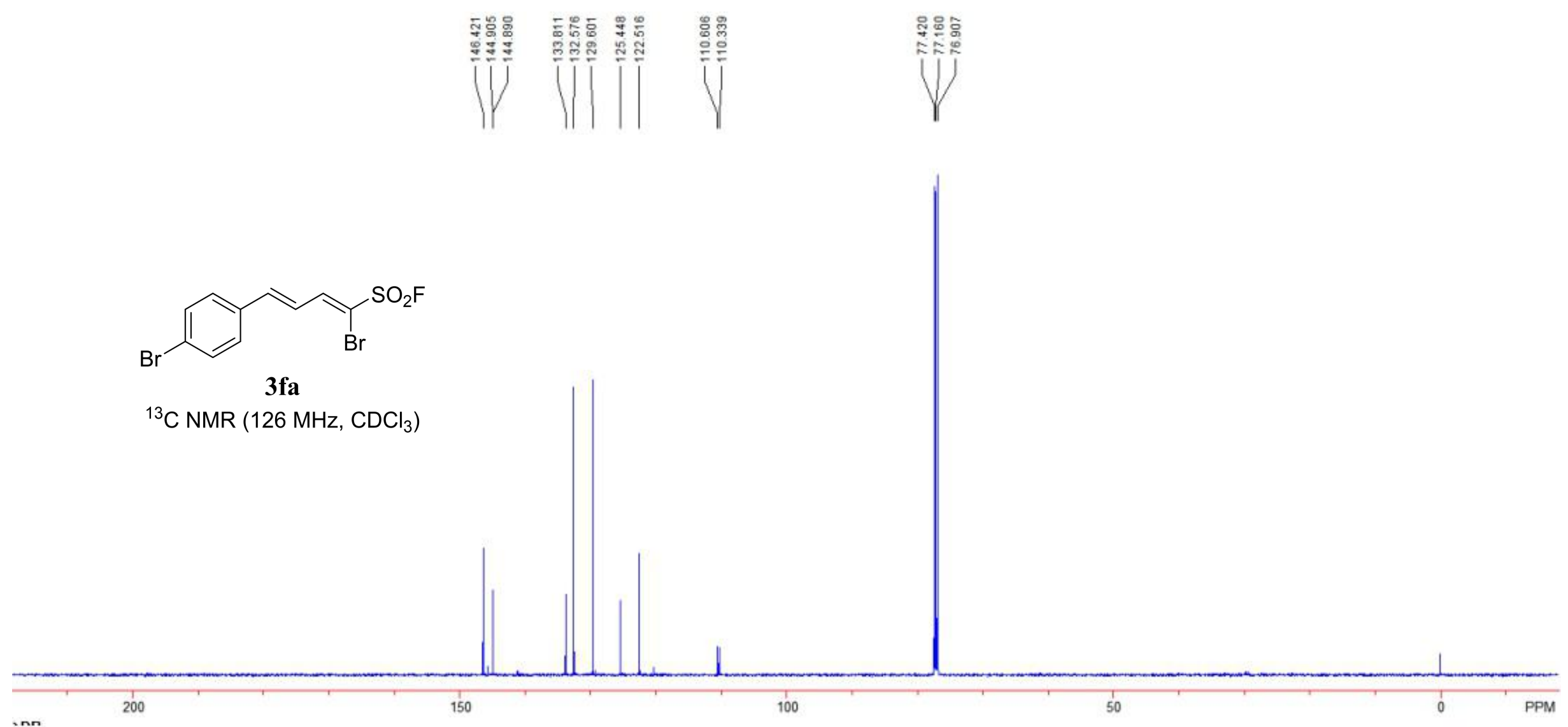




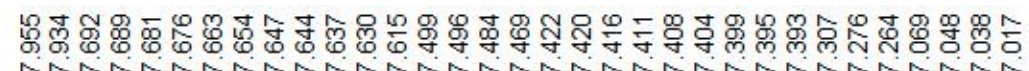

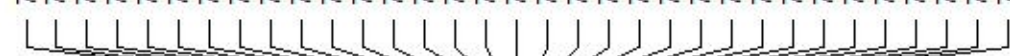

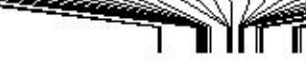

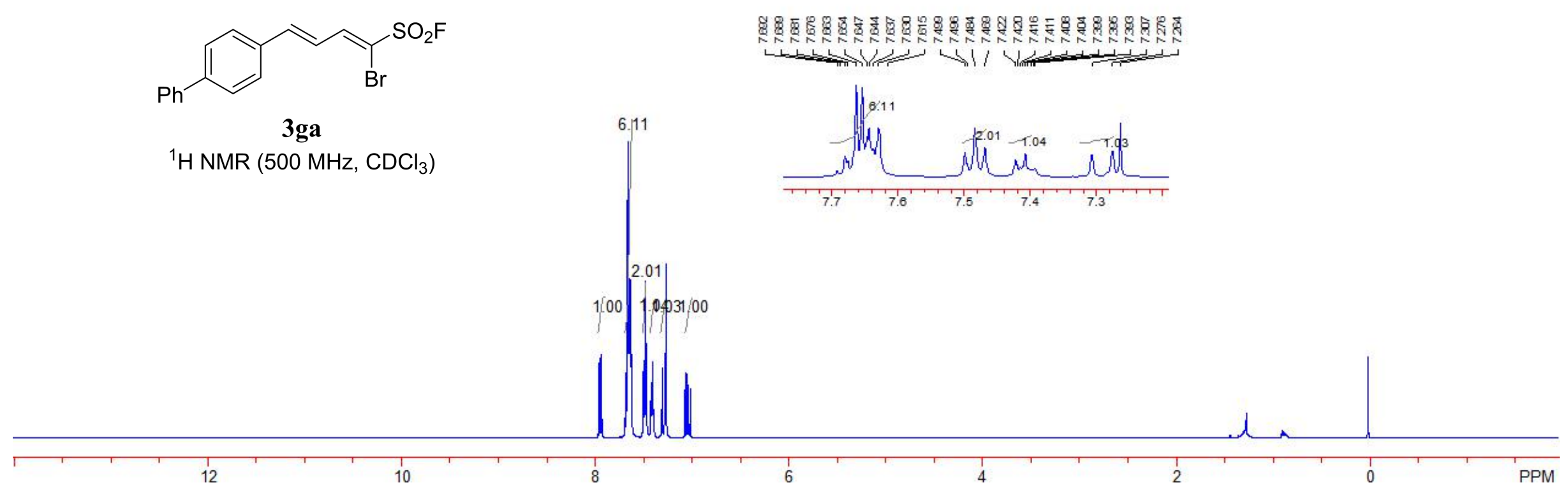




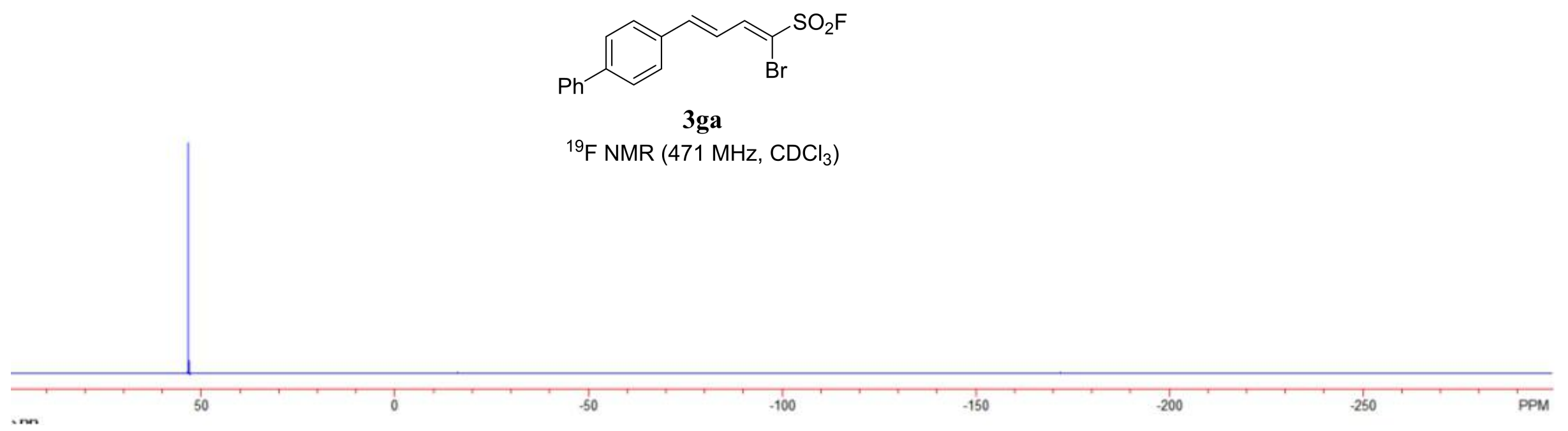




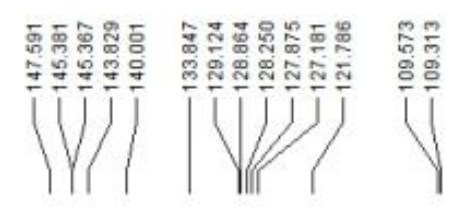

V

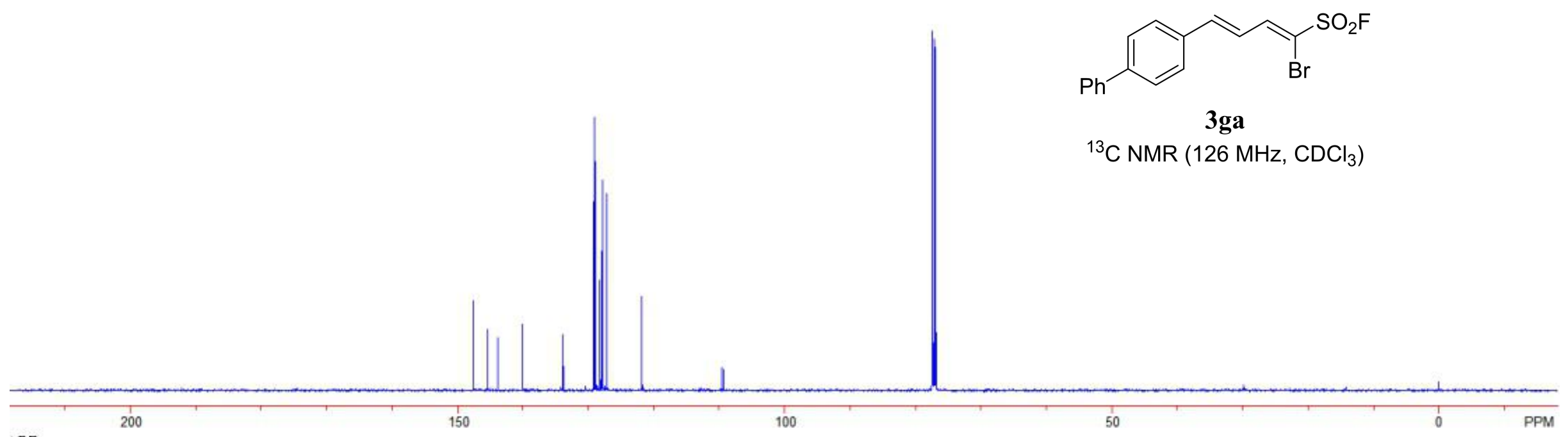




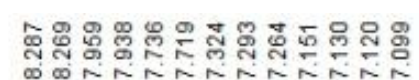

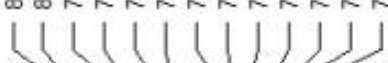

가간

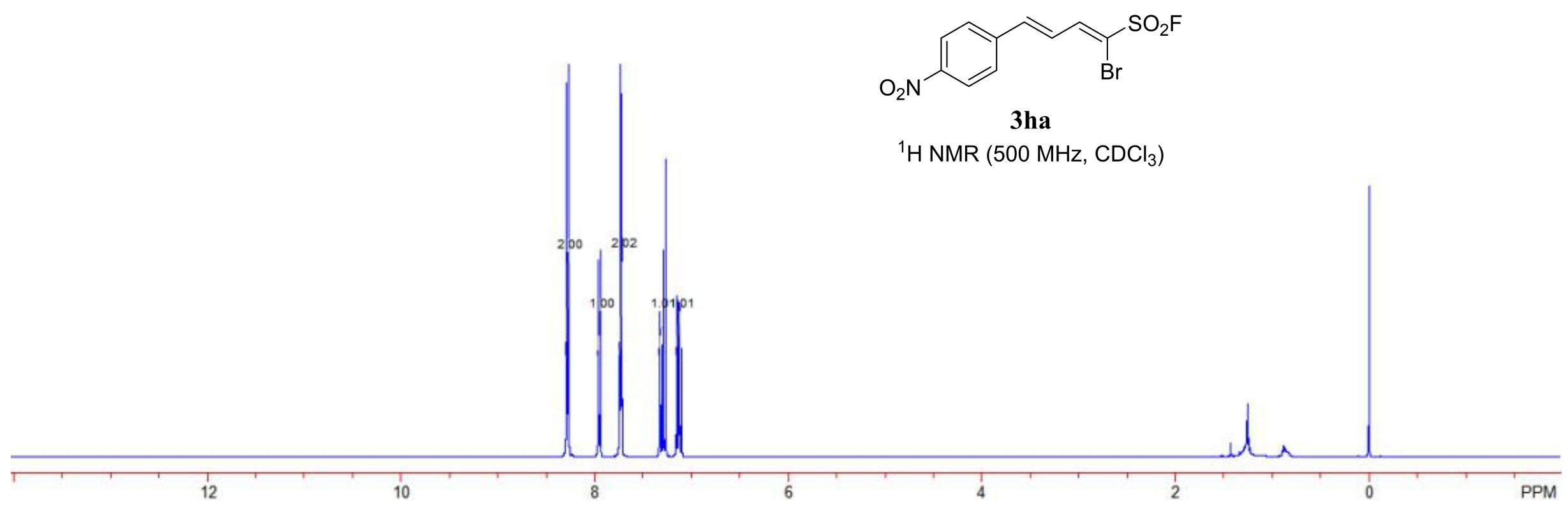



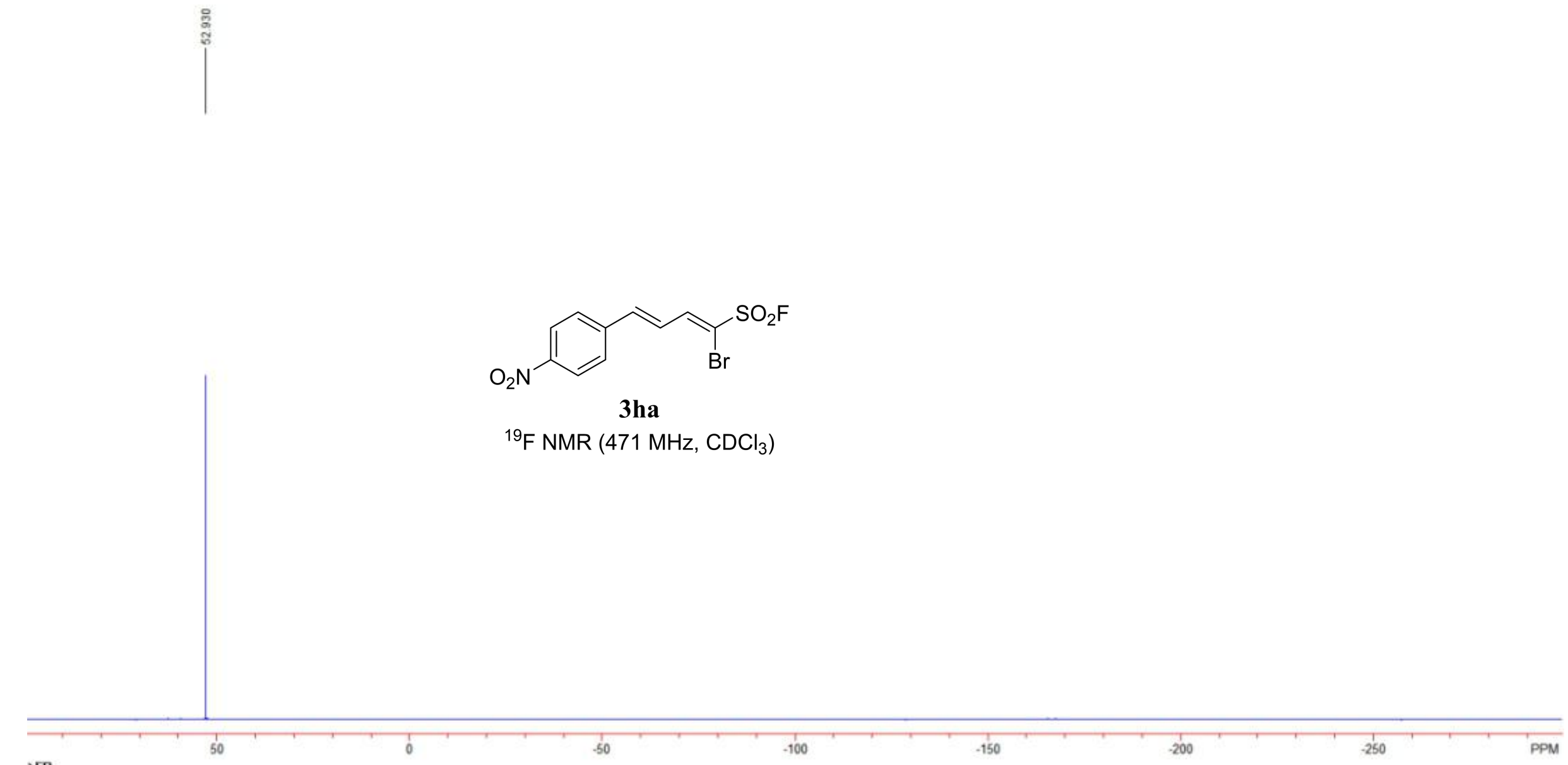

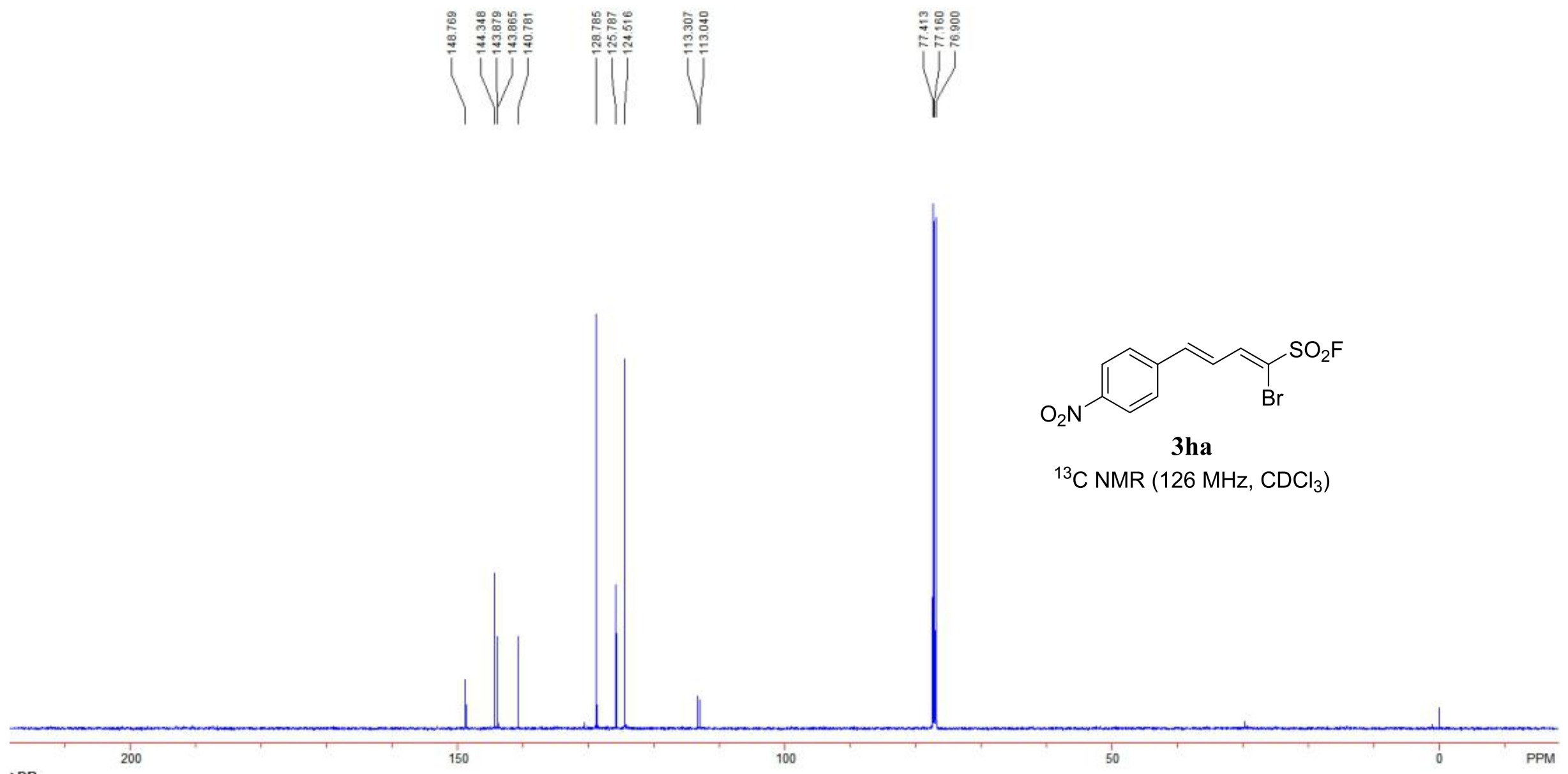


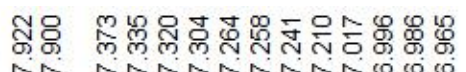

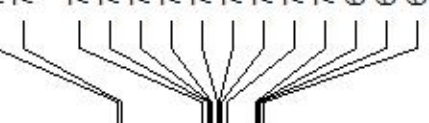

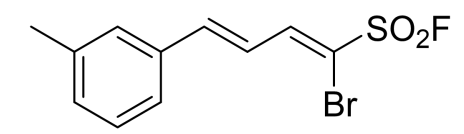

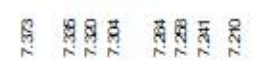

I $|1| \mathrm{Vl}$

喜照照照

$3 \mathbf{i a}$

${ }^{1} \mathrm{H}$ NMR $\left(500 \mathrm{MHz}, \mathrm{CDCl}_{3}\right)$

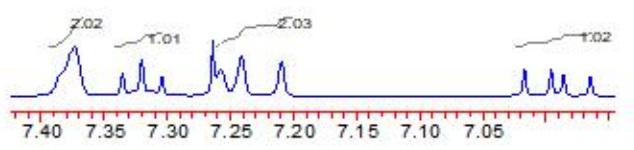

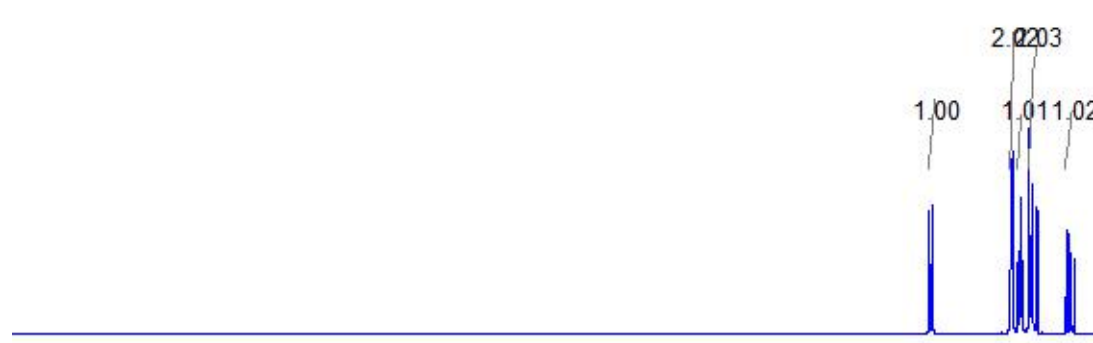




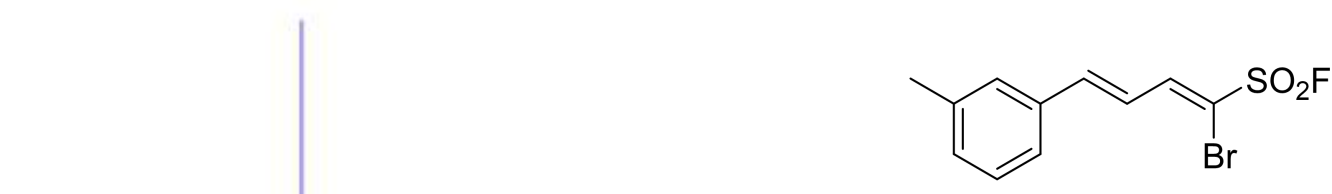

3ia

${ }^{19} \mathrm{~F}$ NMR (471 MHz, $\mathrm{CDCl}_{3}$ )

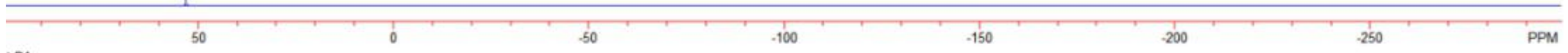



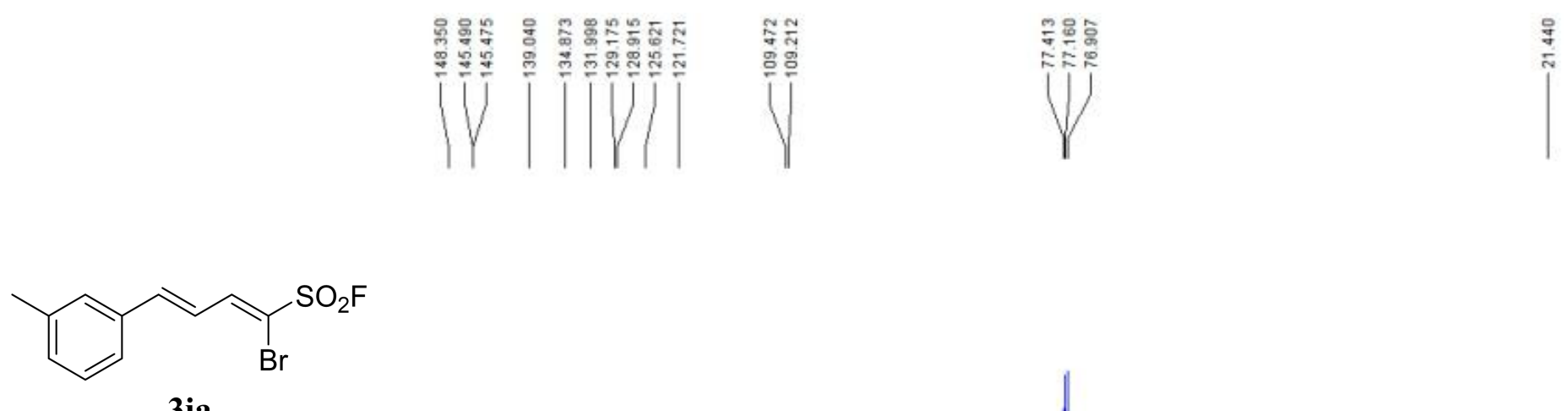

${ }^{13} \mathrm{C}$ NMR (126 MHz, $\mathrm{CDCl}_{3}$ )

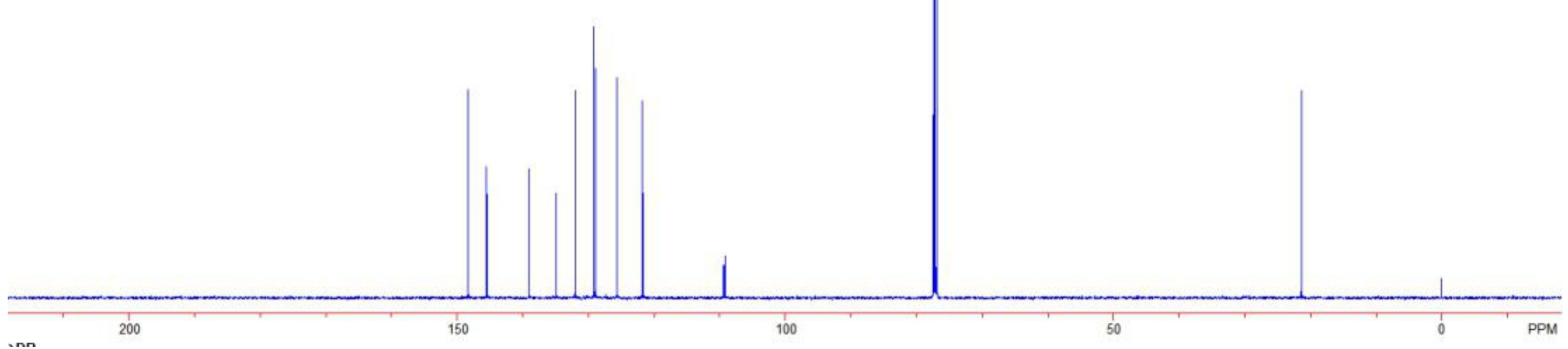




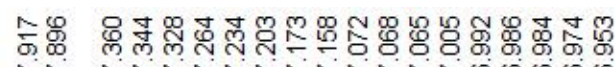

们 Lin

LLUMUUUJ JUJ

$\operatorname{lom}_{7}$ Tाl

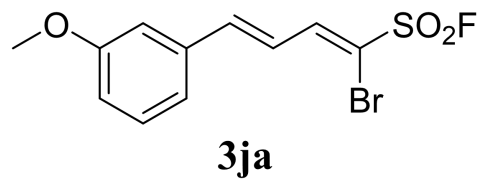

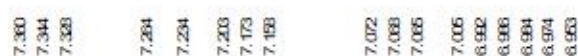

iा

${ }^{1} \mathrm{H}$ NMR (500 MHz, $\left.\mathrm{CDCl}_{3}\right)$

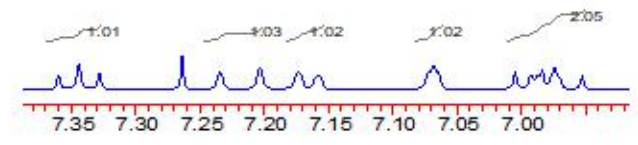

ili

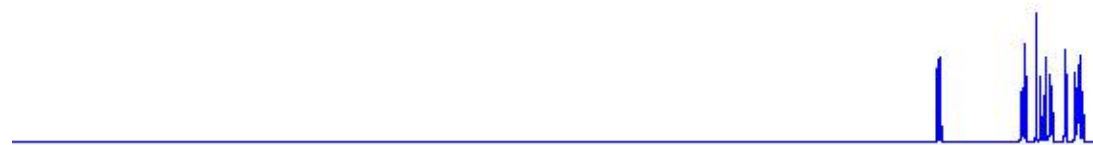




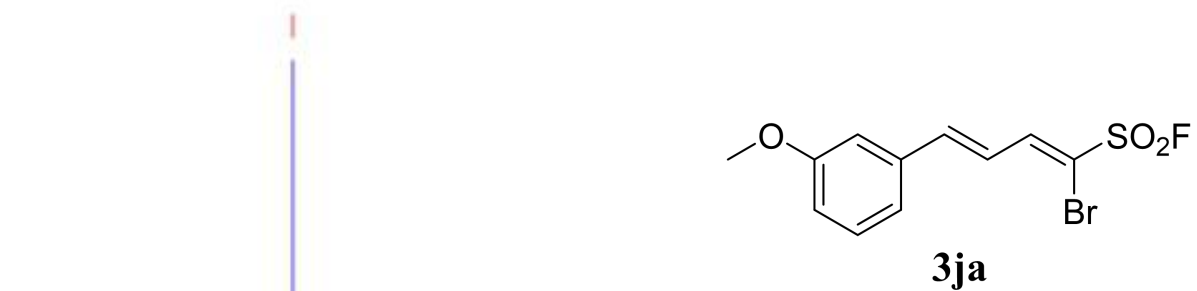

${ }^{19} \mathrm{~F} \mathrm{NMR}\left(471 \mathrm{MHz}, \mathrm{CDCl}_{3}\right)$ 


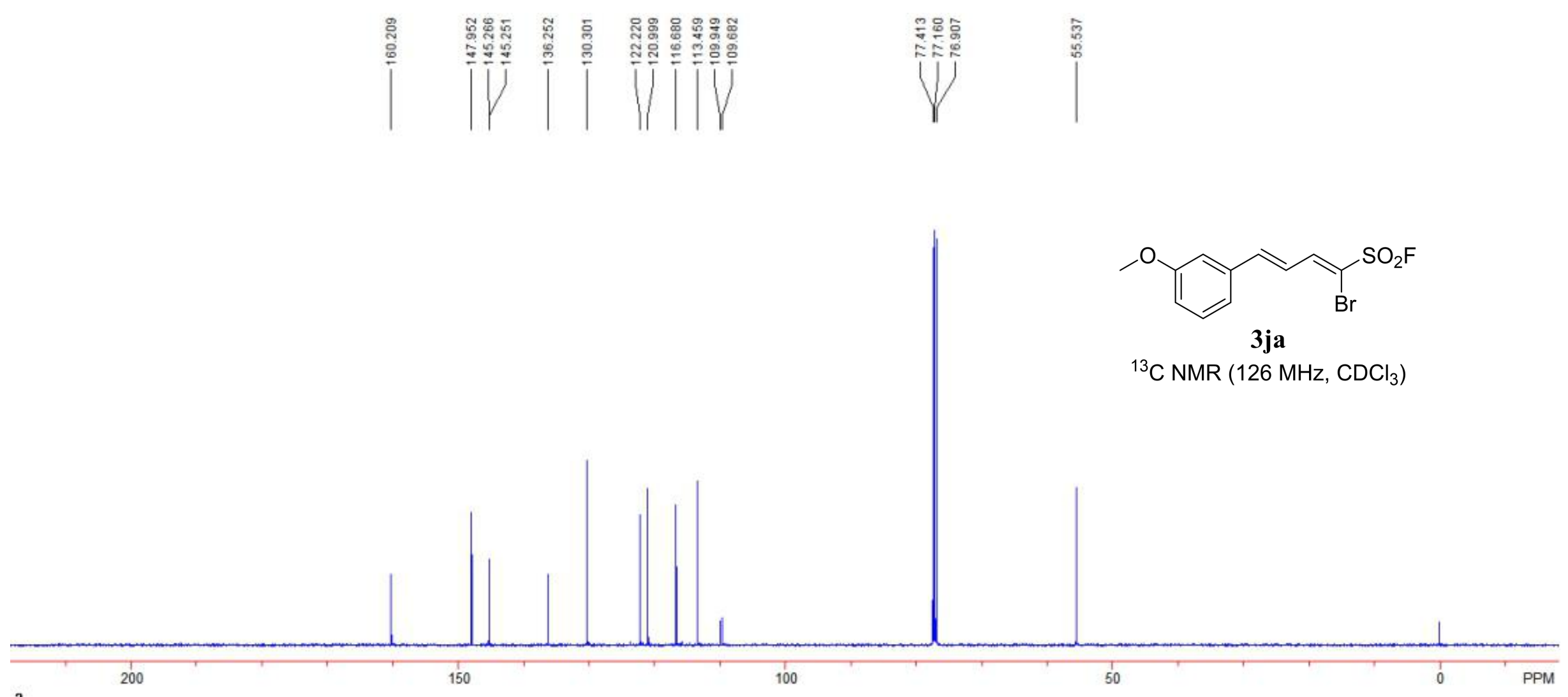




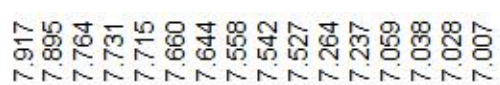

11111

$+171$

\section{me}

(1)

$\overbrace{\mathrm{Br}}^{\mathrm{SO}_{2} \mathrm{~F}}$

$3 \mathbf{k a}$

${ }^{1} \mathrm{H}$ NMR (500 MHz, $\mathrm{CDCl}_{3}$ )
1. $\operatorname{agn} 211.01 .01$

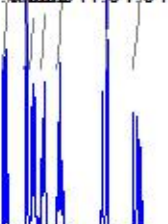




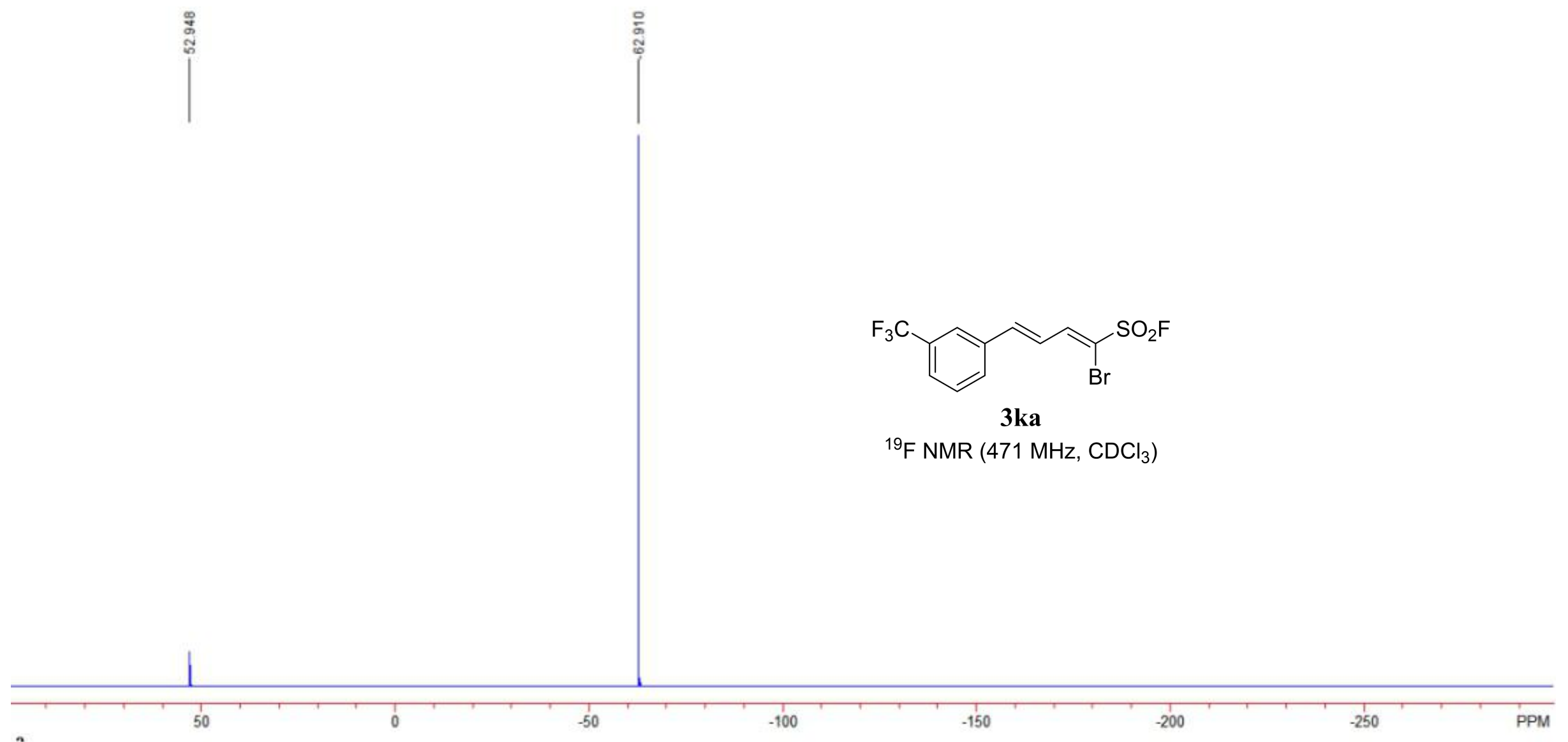




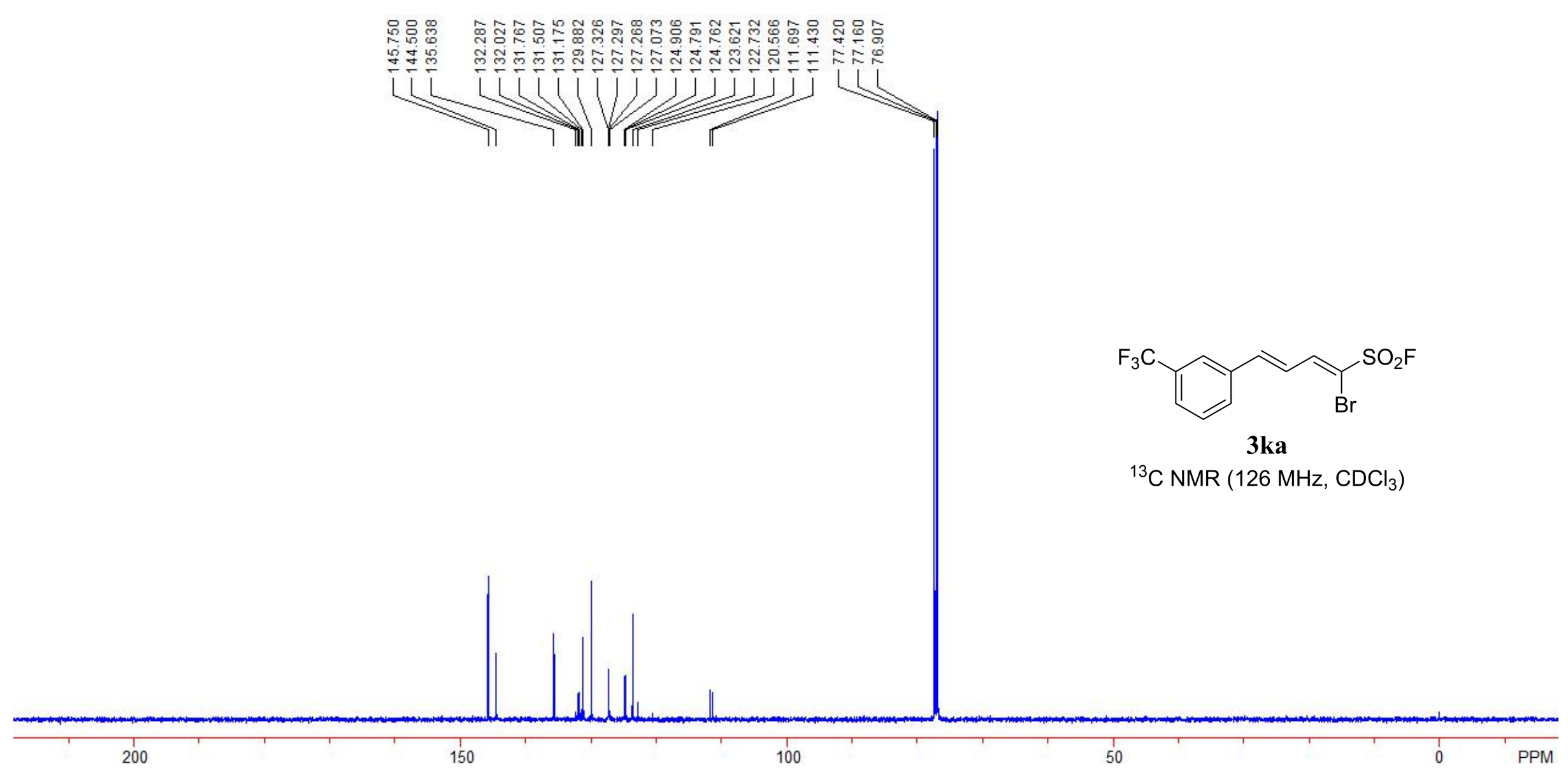




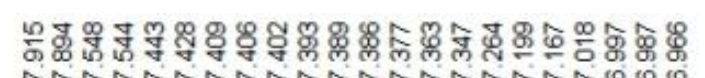

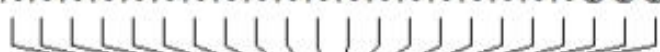

( 7 - 1111

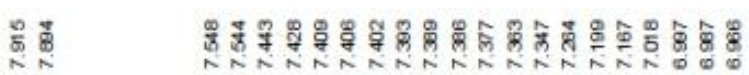

tron

$\mathrm{Crr}_{\mathrm{Br}}^{\mathrm{SO}_{2} \mathrm{~F}}$

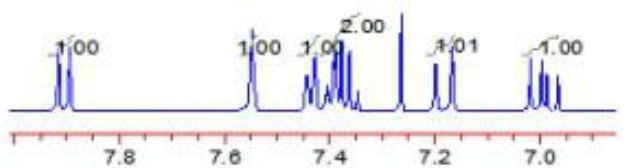

3la

${ }^{1} \mathrm{H} \mathrm{NMR}\left(500 \mathrm{MHz}, \mathrm{CDCl}_{3}\right)$

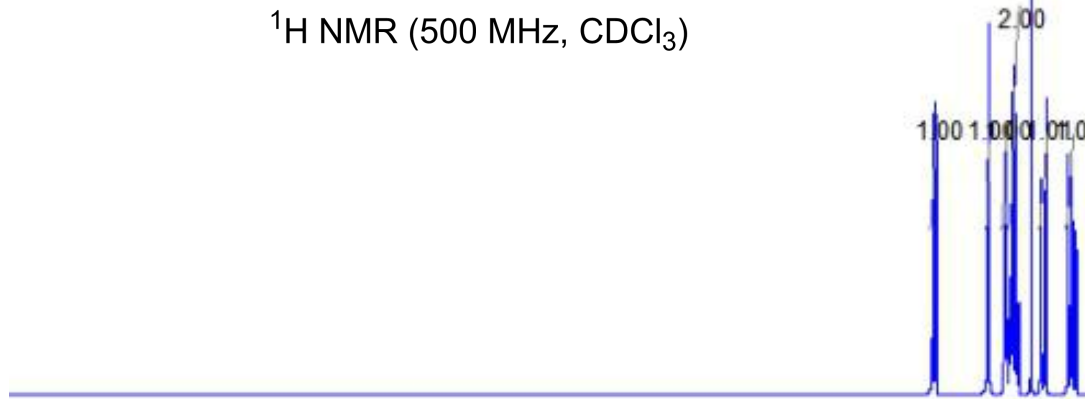

12

10

2

PPM 


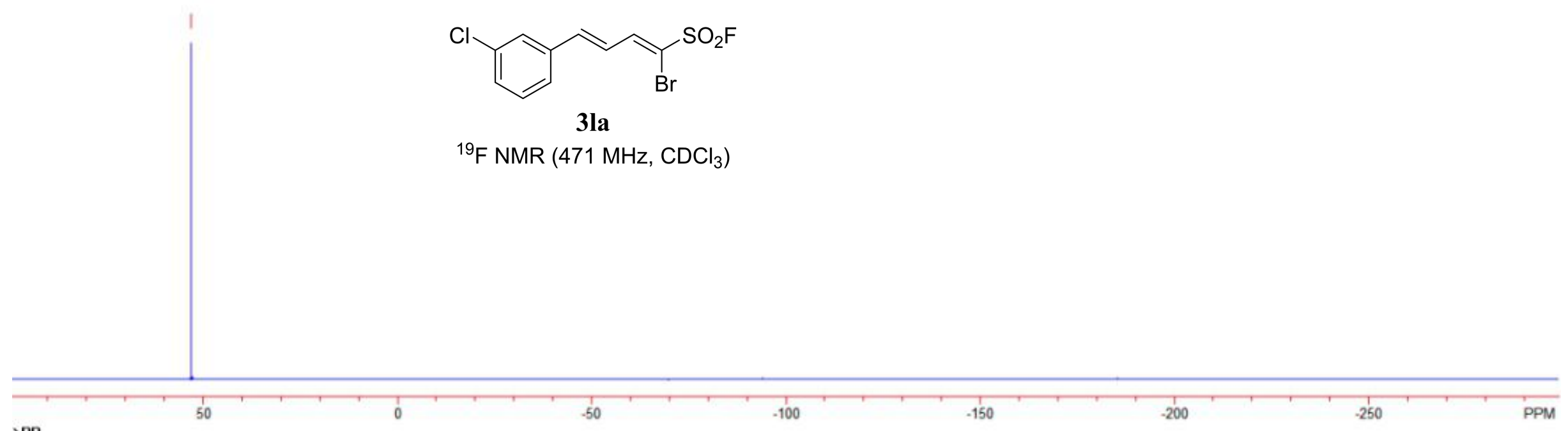




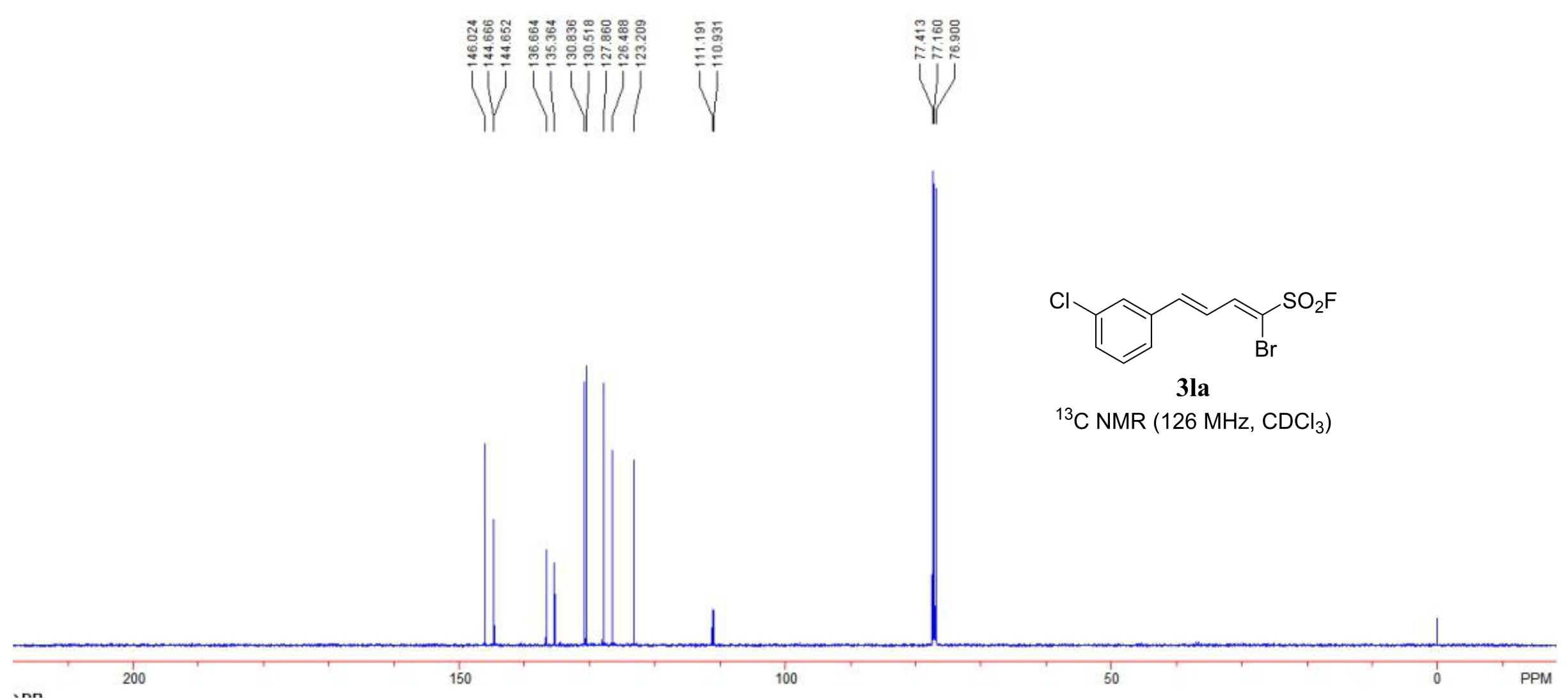




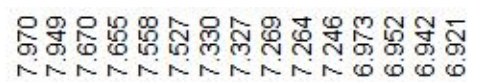

1)

I Ii Vir
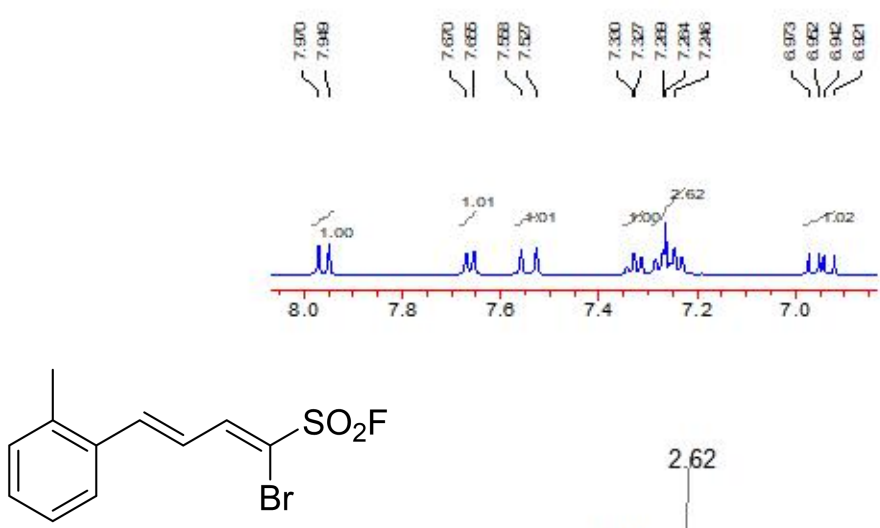

$3 \mathrm{ma}$

${ }^{1} \mathrm{H}$ NMR $\left(500 \mathrm{MHz}, \mathrm{CDCl}_{3}\right)$

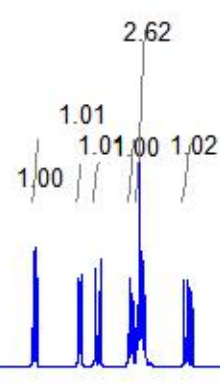

12

10

6

PPM 


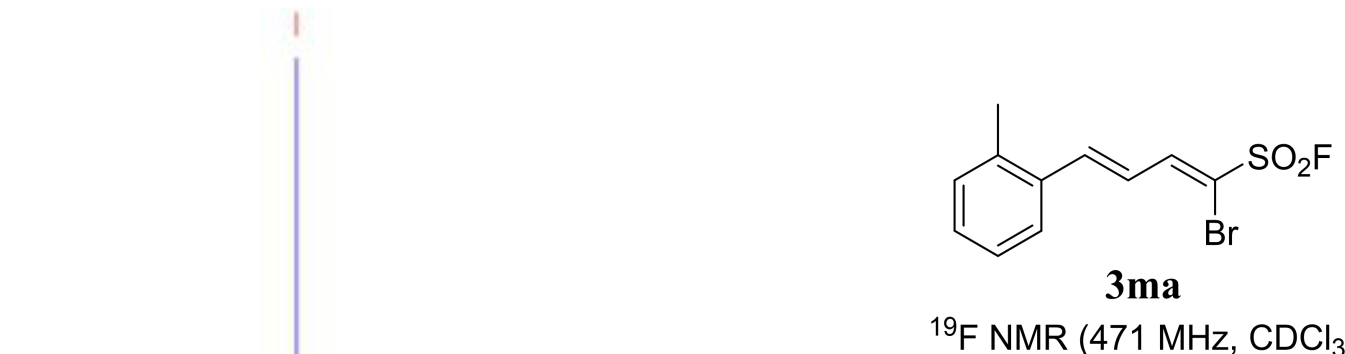

${ }^{19} \mathrm{~F} \mathrm{NMR}\left(471 \mathrm{MHz}, \mathrm{CDCl}_{3}\right)$ 

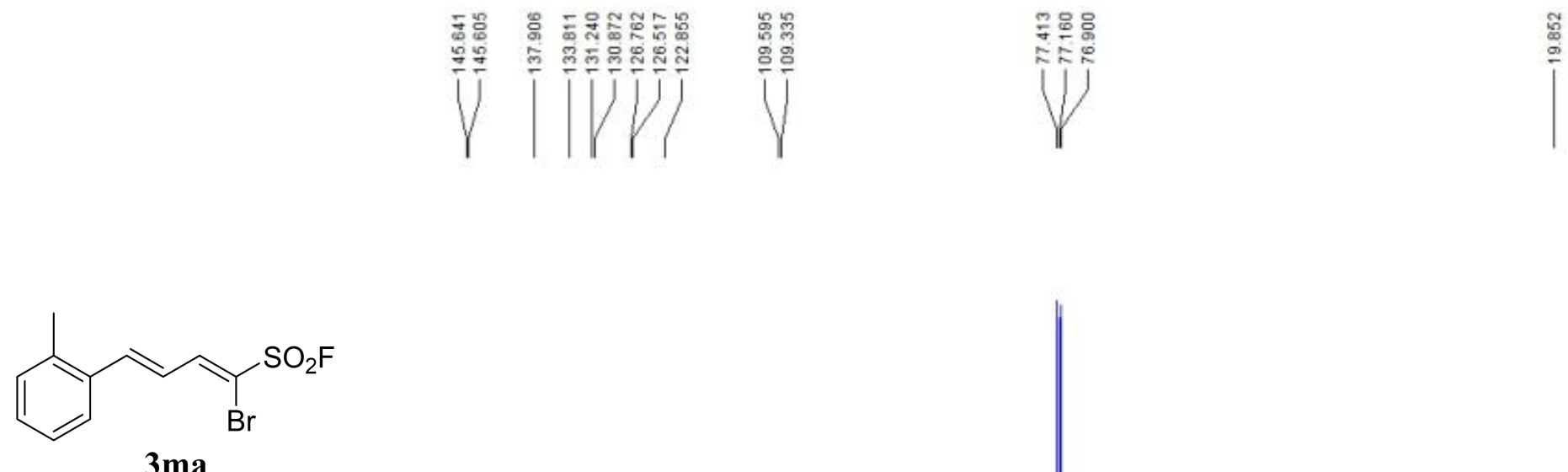

${ }^{13} \mathrm{C}$ NMR (126 MHz, $\mathrm{CDCl}_{3}$ )

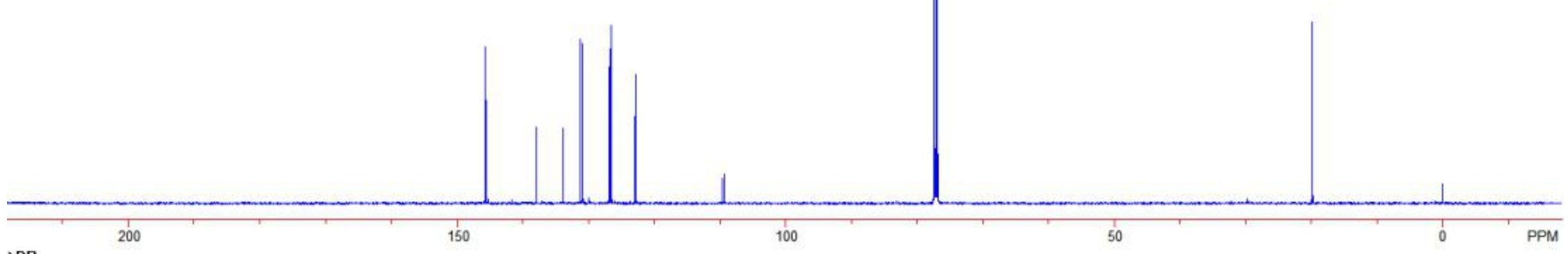




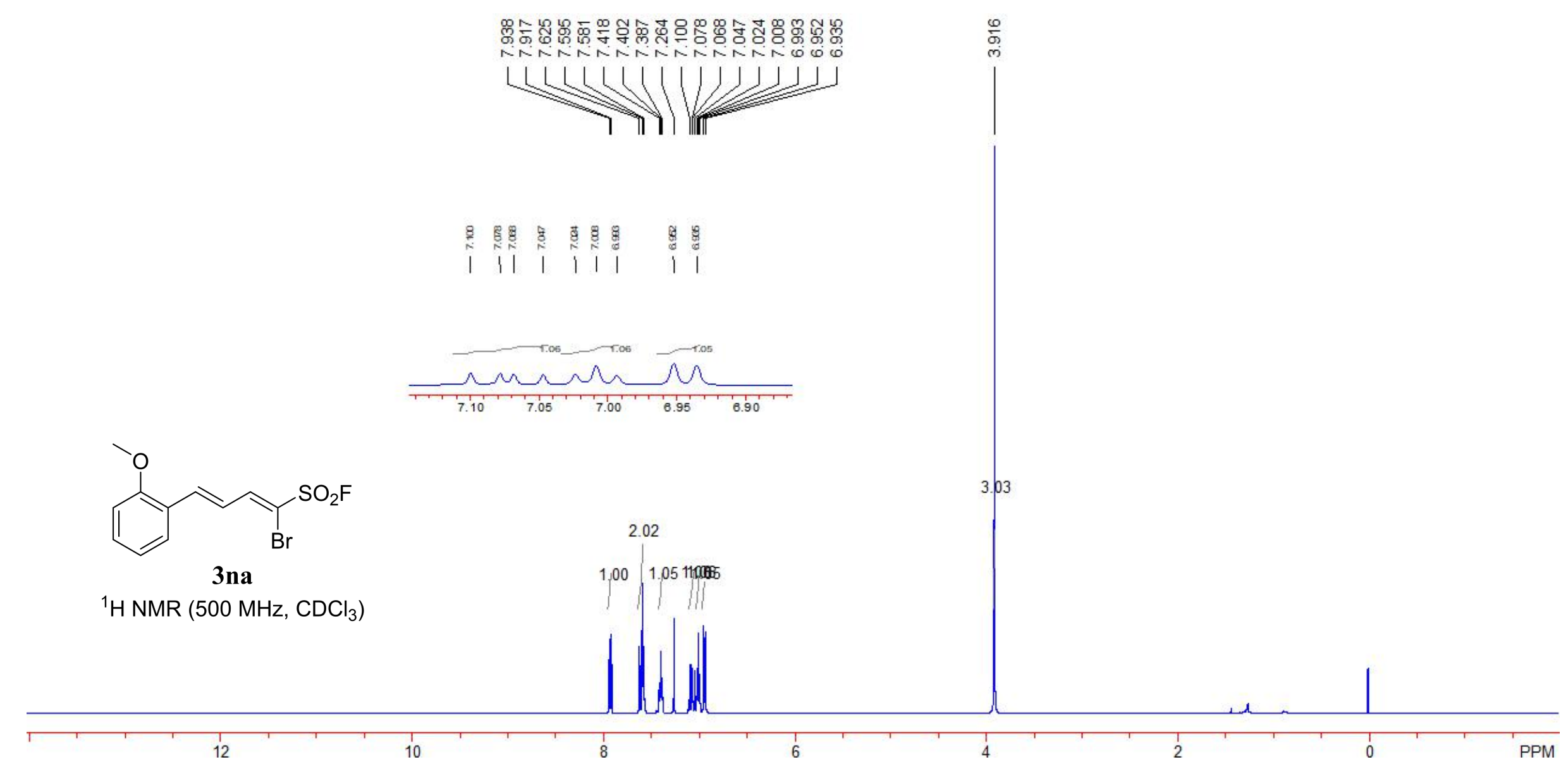



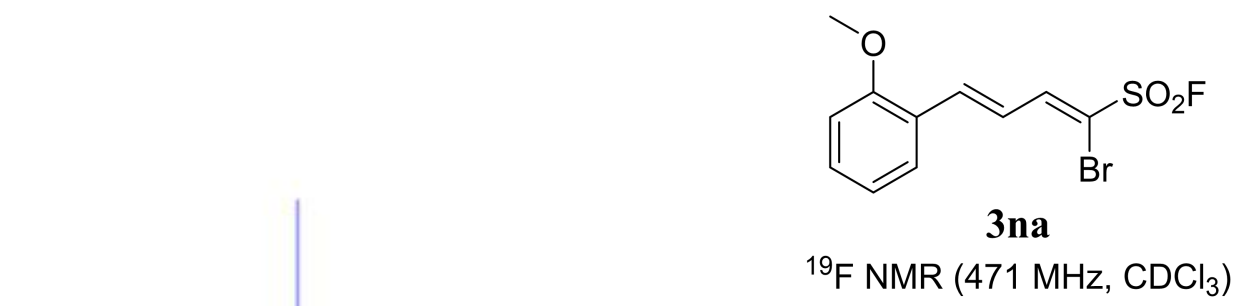

${ }^{19} \mathrm{~F} \mathrm{NMR}\left(471 \mathrm{MHz}, \mathrm{CDCl}_{3}\right)$ 

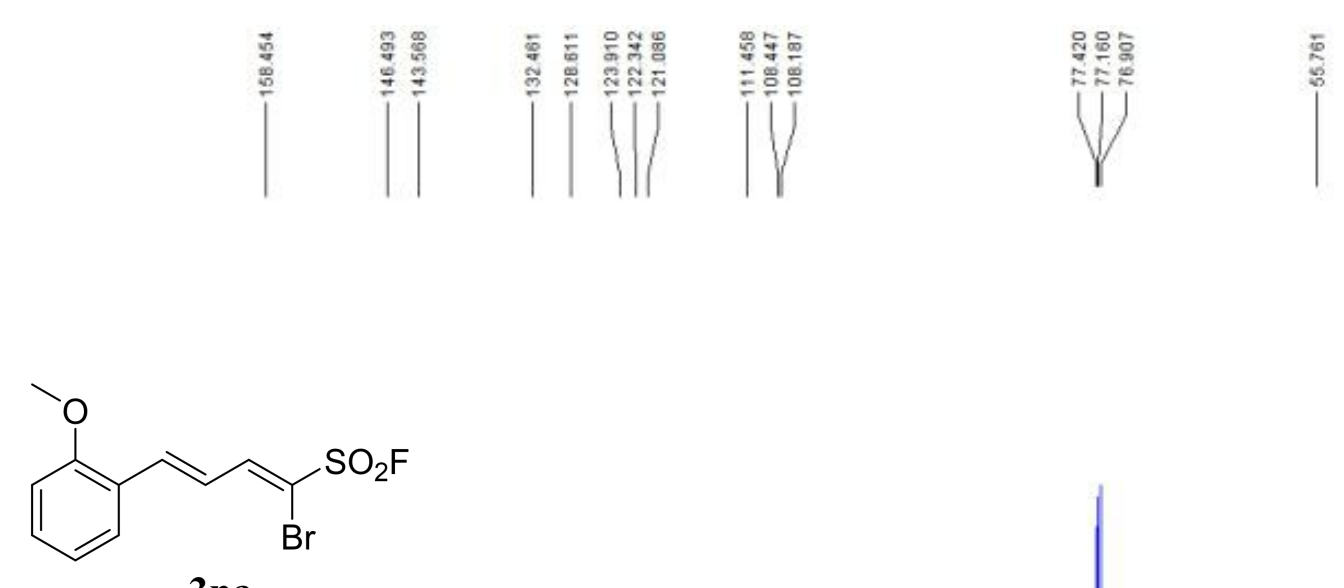

3na

${ }^{13} \mathrm{C}$ NMR (126 MHz, $\mathrm{CDCl}_{3}$ )

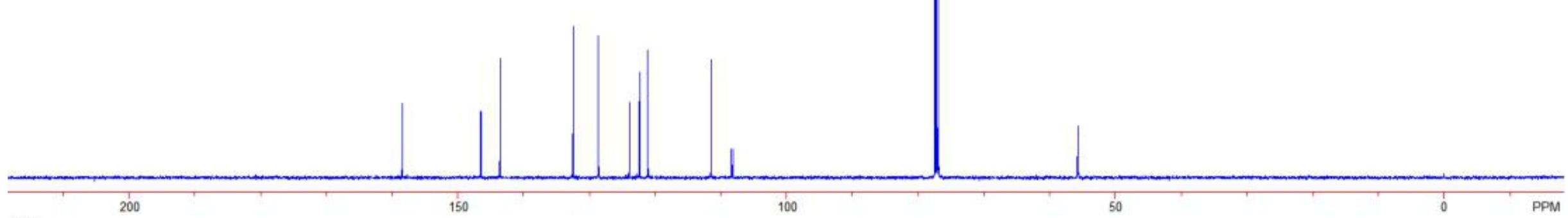



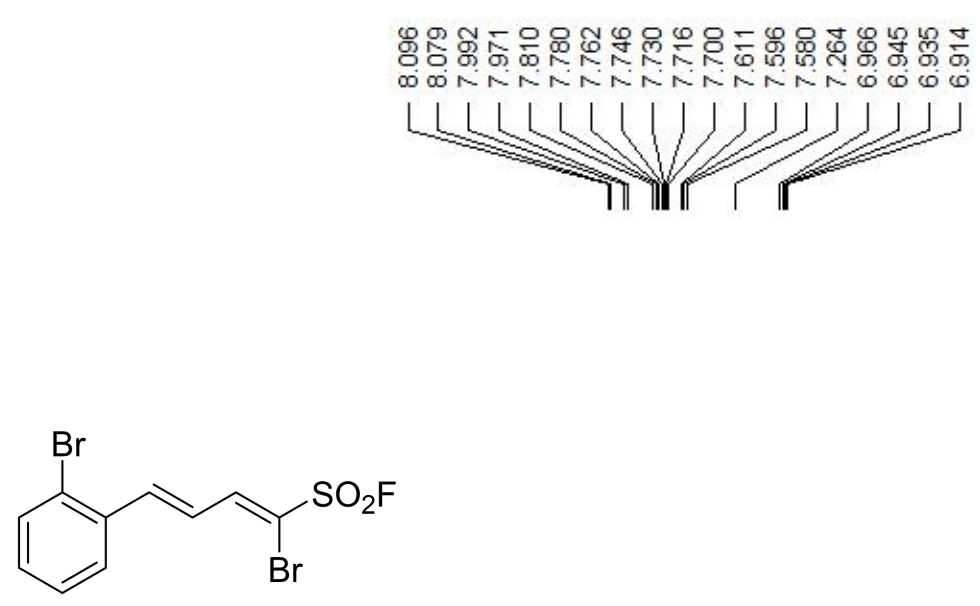

3oa

${ }^{1} \mathrm{H}$ NMR $\left(500 \mathrm{MHz}, \mathrm{CDCl}_{3}\right)$

3.04

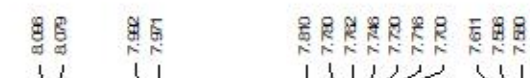

$1 / 11 \quad 1111 / 4511$
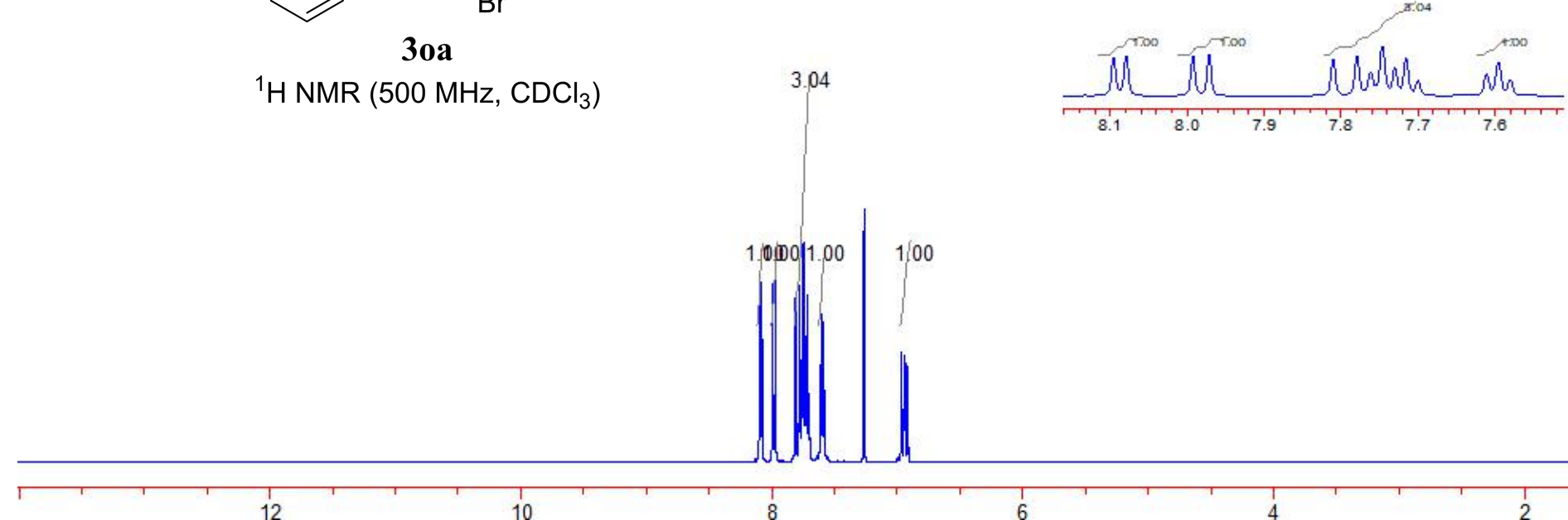

12

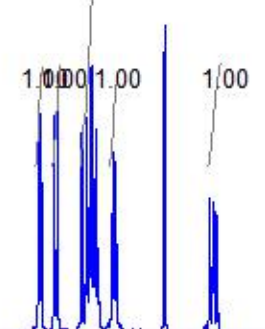

PPM 

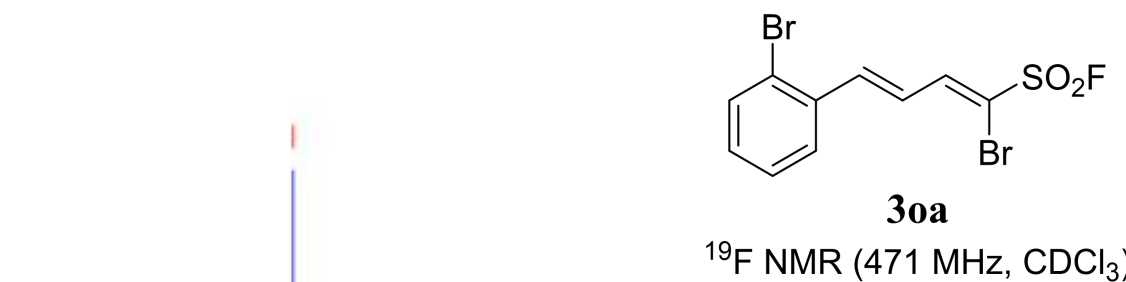

${ }^{19} \mathrm{~F}$ NMR $\left(471 \mathrm{MHz}, \mathrm{CDCl}_{3}\right)$

... 50 


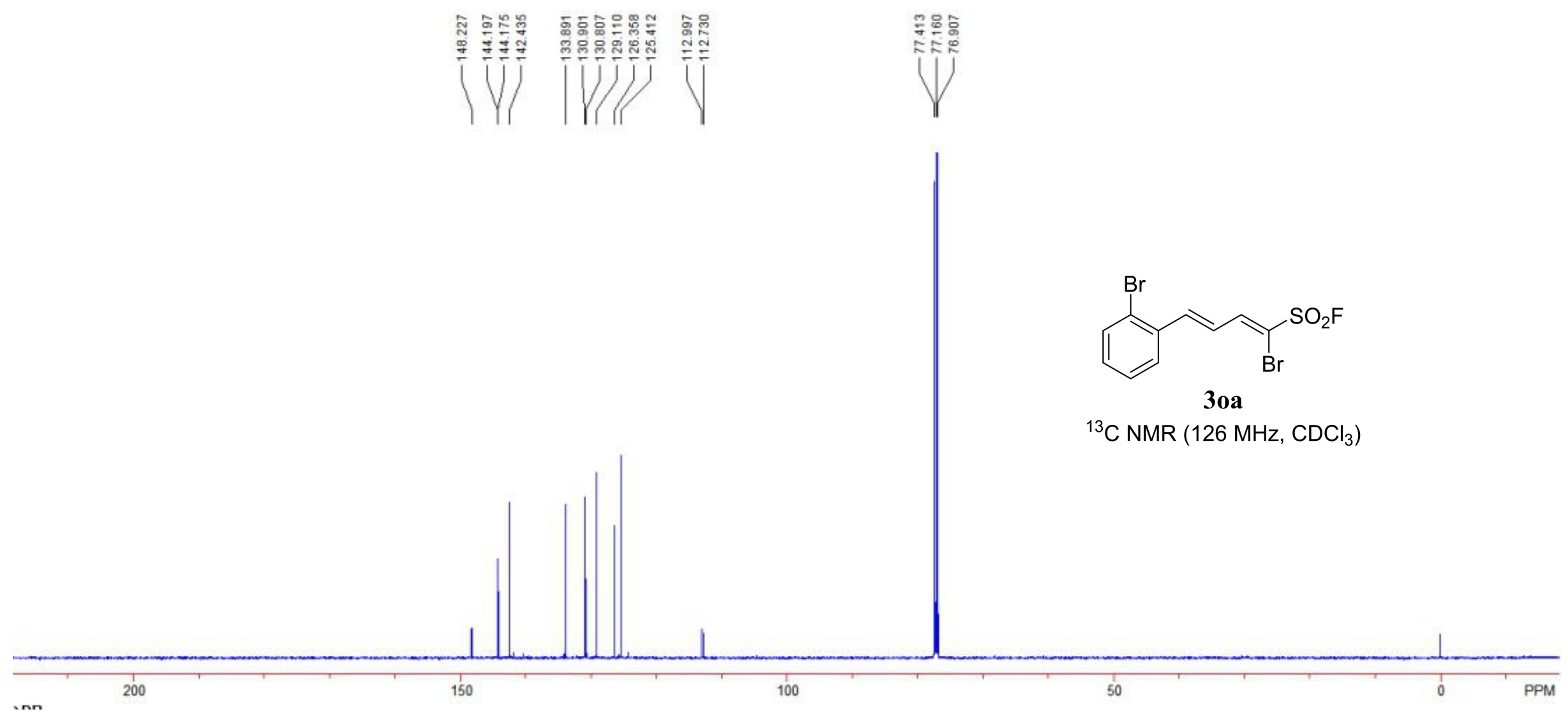




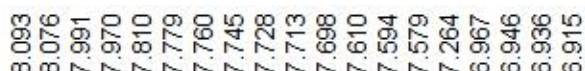 \\ Lu LU U U J J U J

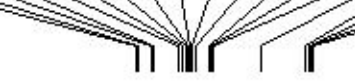

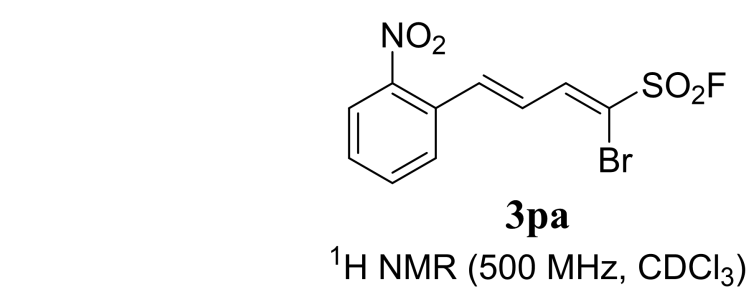

${ }^{1} \mathrm{H}$ NMR $\left(500 \mathrm{MHz}, \mathrm{CDCl}_{3}\right)$

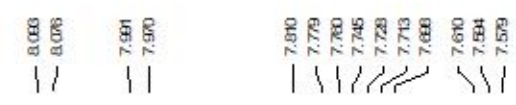
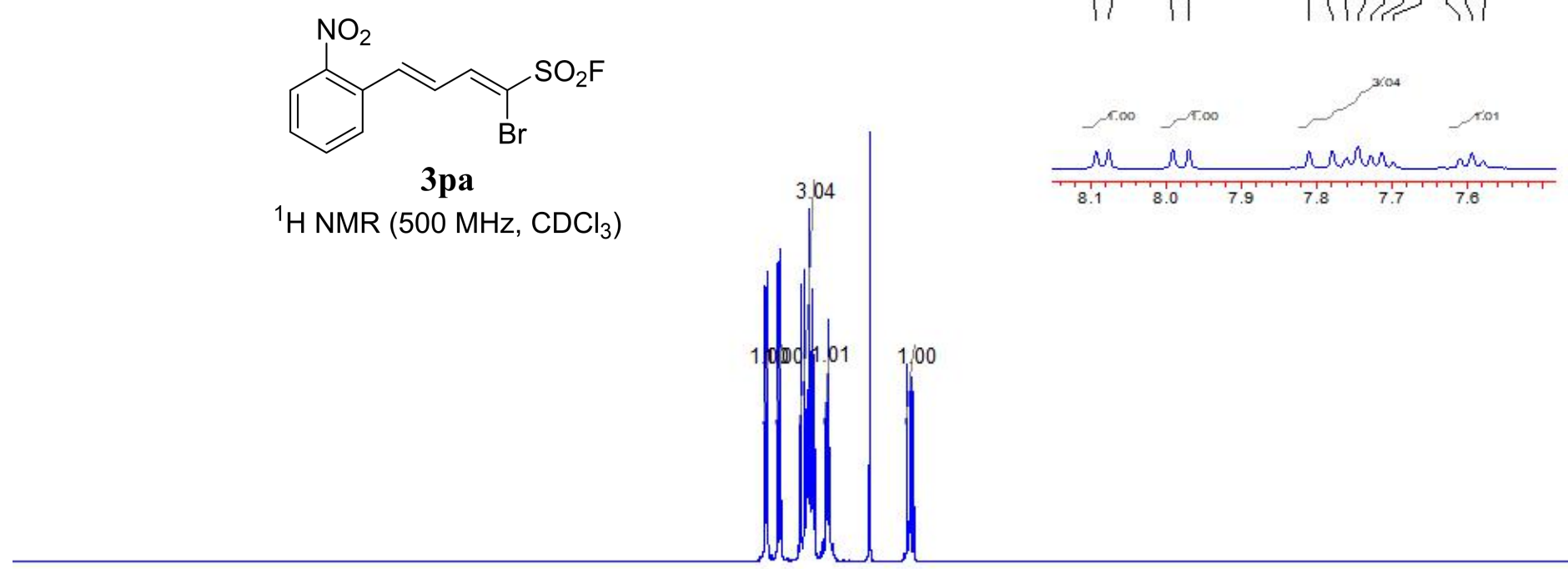


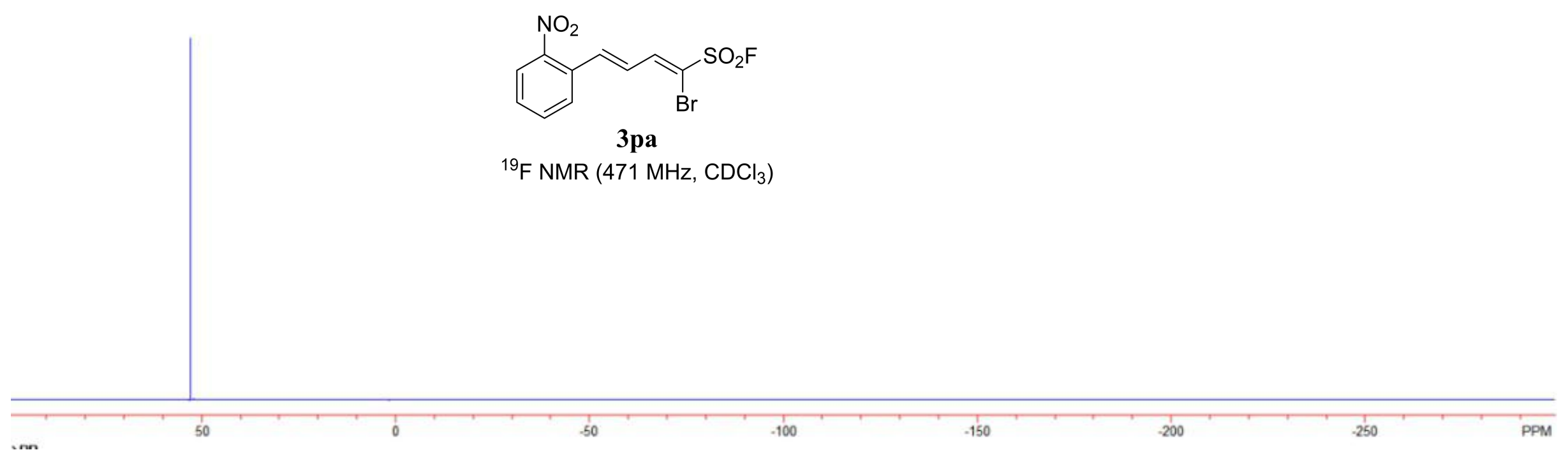




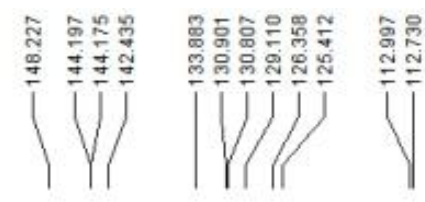

$\mathrm{NO}_{2}$

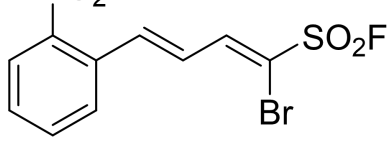

3 pa

${ }^{13} \mathrm{C} \mathrm{NMR} \mathrm{(126} \mathrm{MHz,} \mathrm{CDCl}_{3}$ )

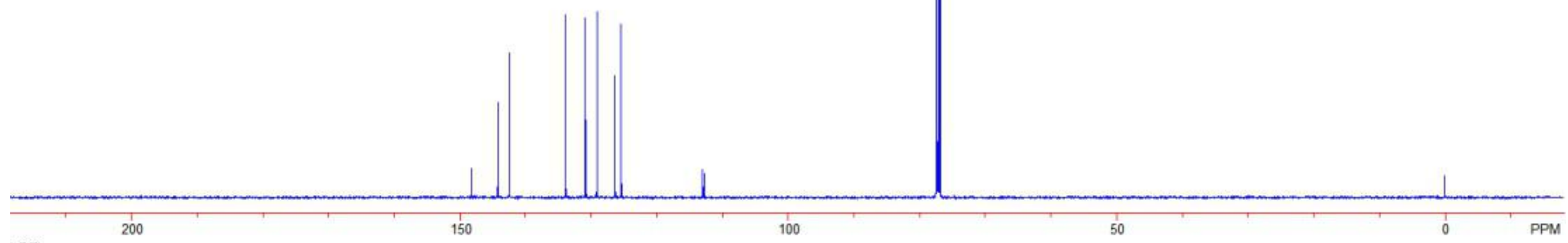



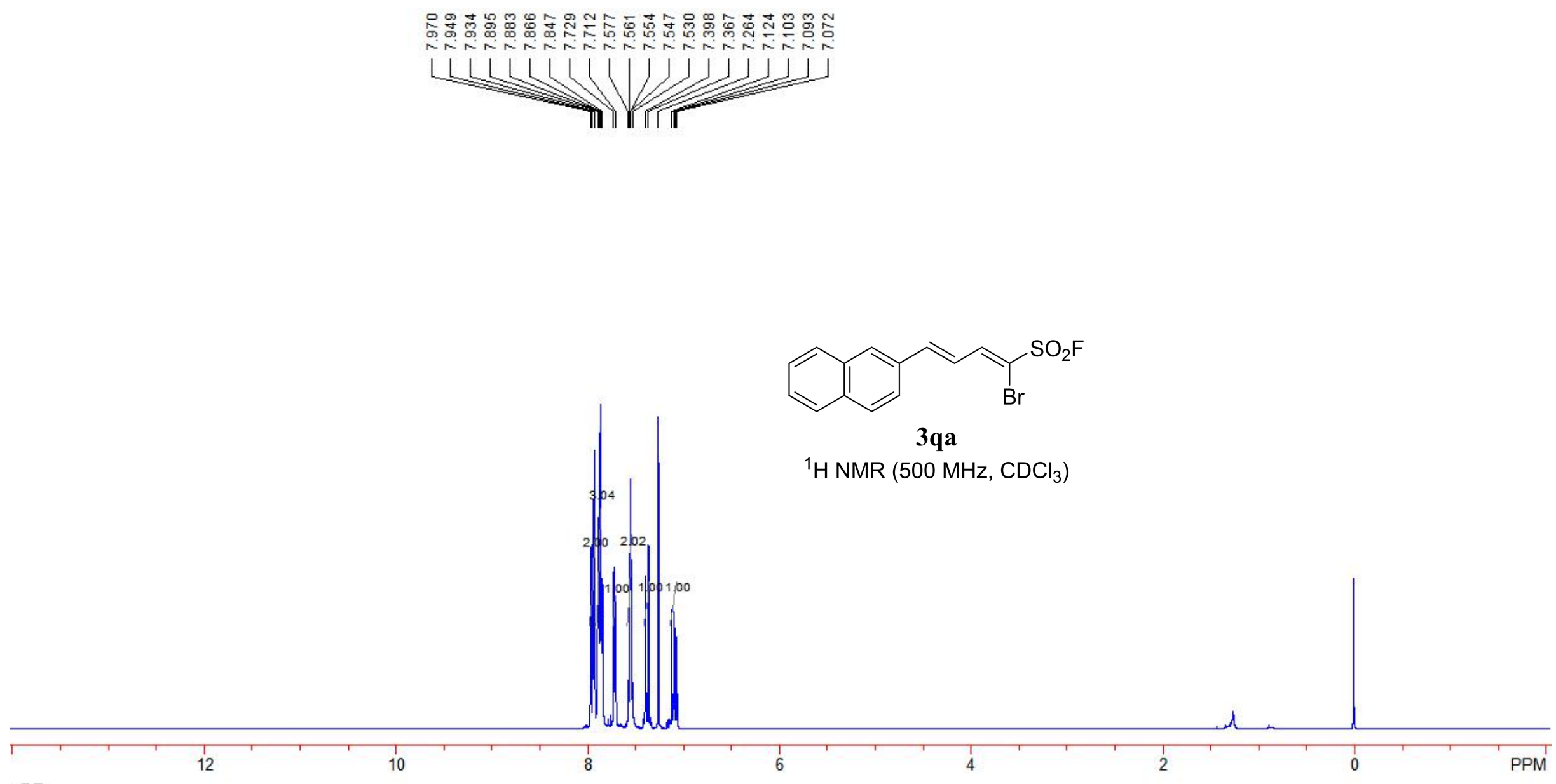


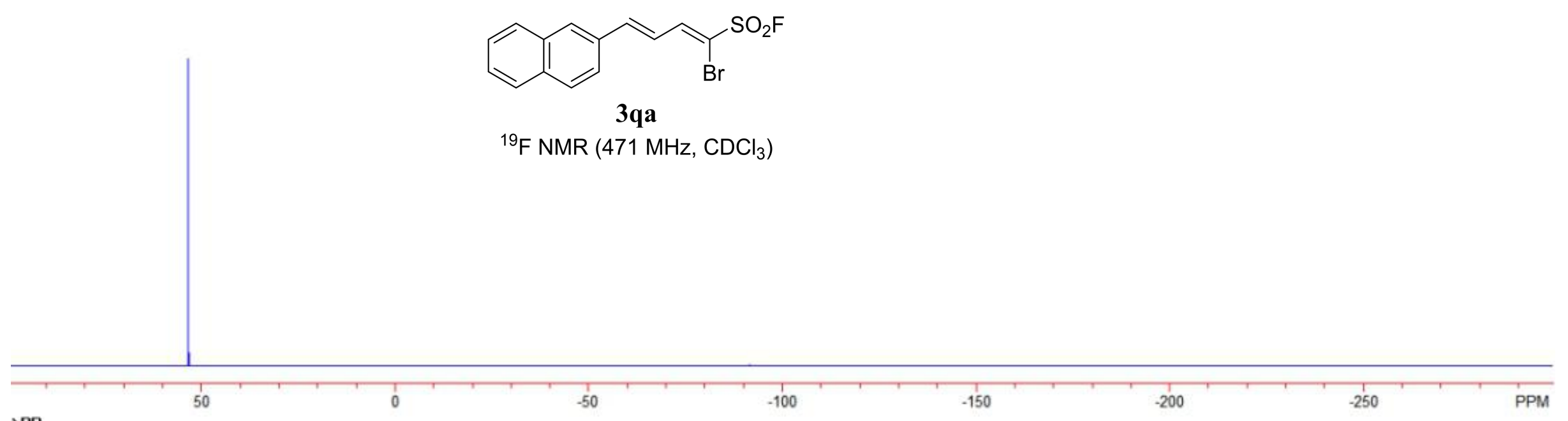




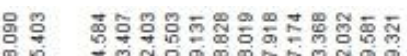

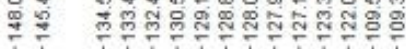

(U)

舟

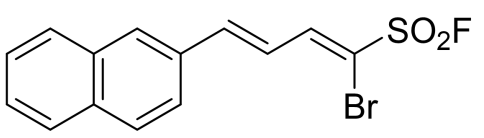

$3 q a$

${ }^{13} \mathrm{C}$ NMR (126 MHz, $\left.\mathrm{CDCl}_{3}\right)$

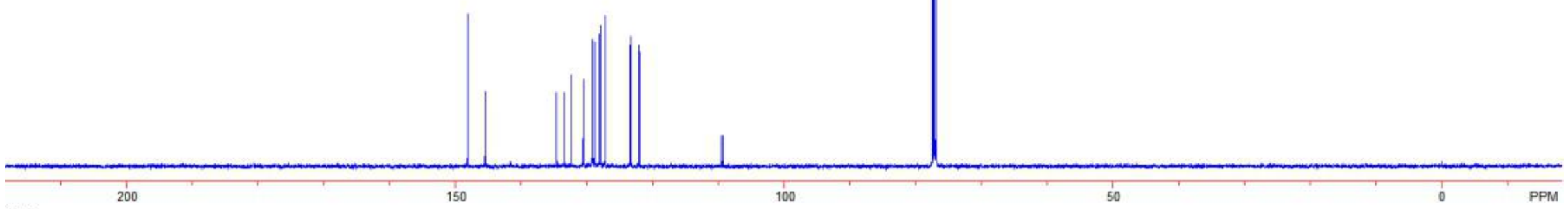




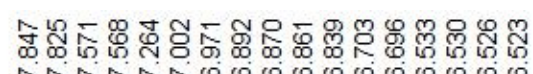

NANAN

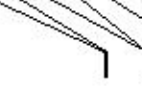
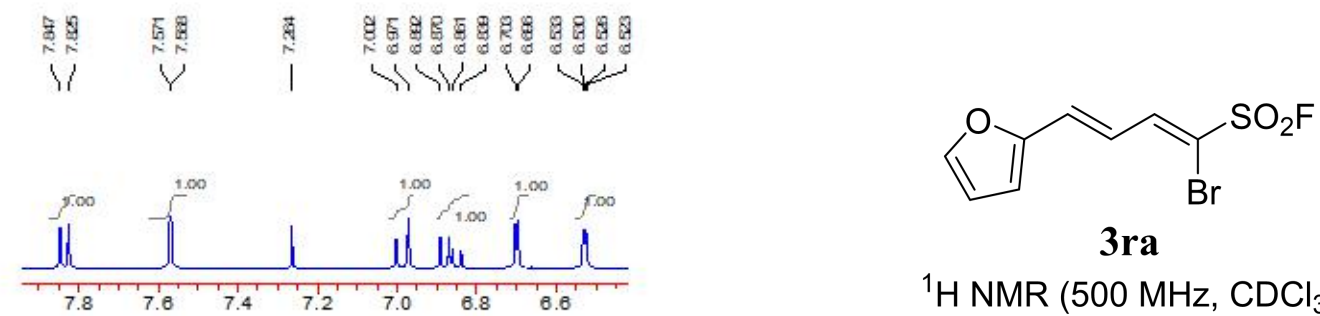

${ }^{1} \mathrm{H}$ NMR $\left(500 \mathrm{MHz}, \mathrm{CDCl}_{3}\right)$

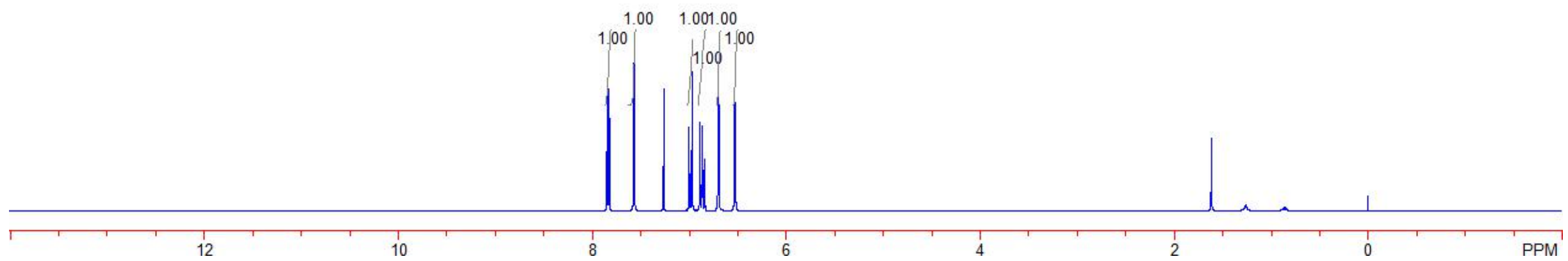




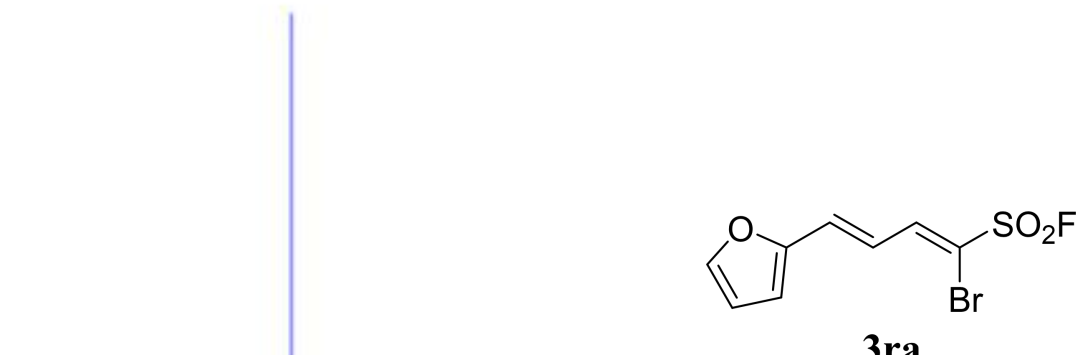

${ }^{19} \mathrm{~F} \mathrm{NMR}\left(471 \mathrm{MHz}, \mathrm{CDCl}_{3}\right)$

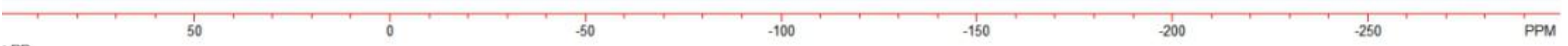



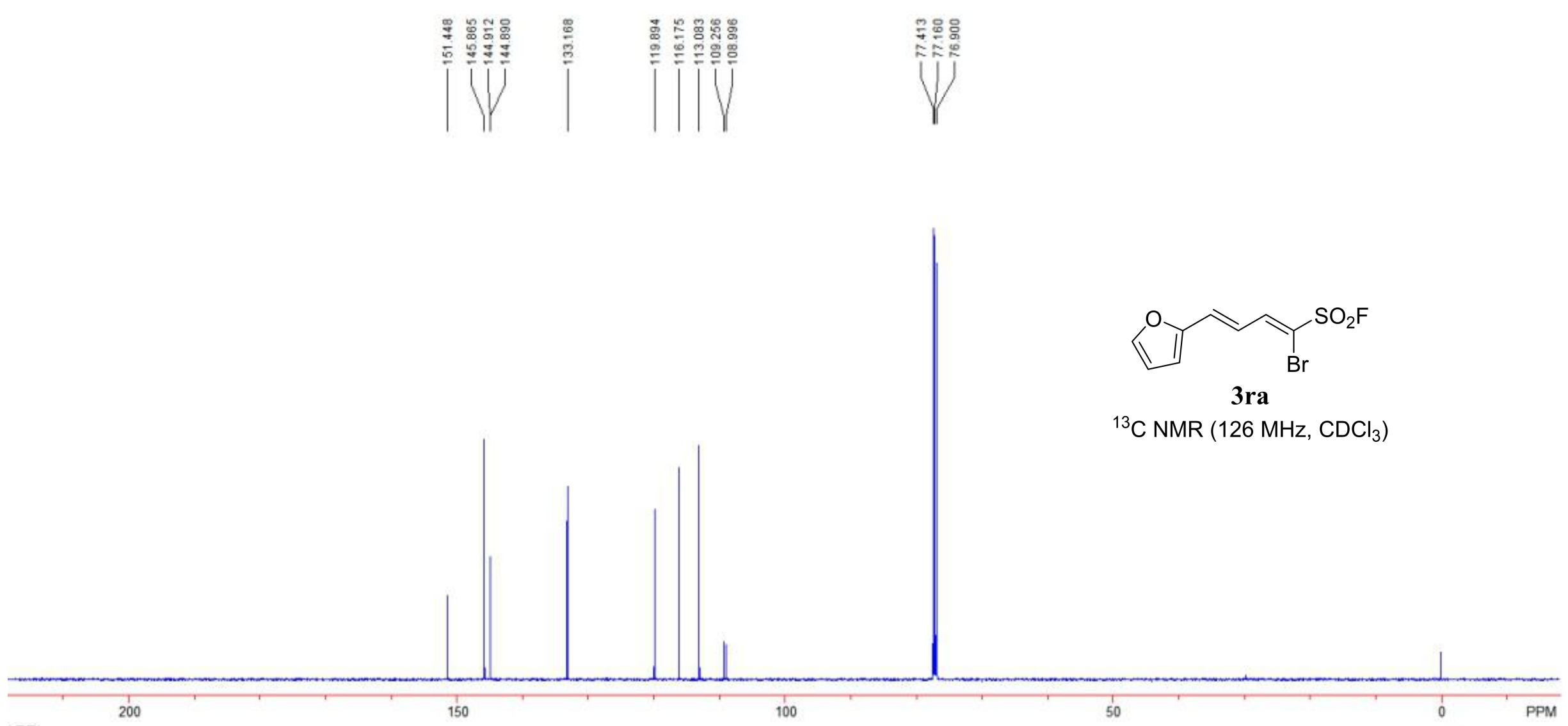


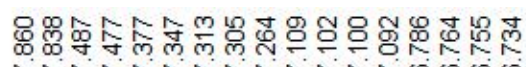

UMW HIIIU

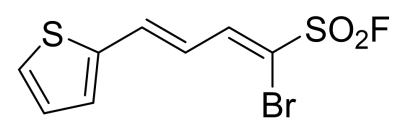

3sa

${ }^{1} \mathrm{H} \mathrm{NMR}\left(500 \mathrm{MHz}, \mathrm{CDCl}_{3}\right)$

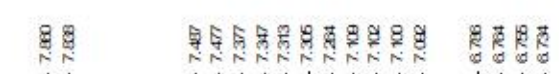

पे प्राजण पर

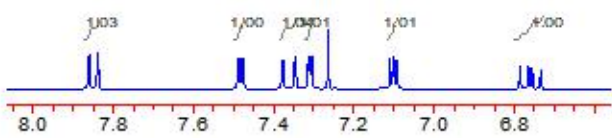

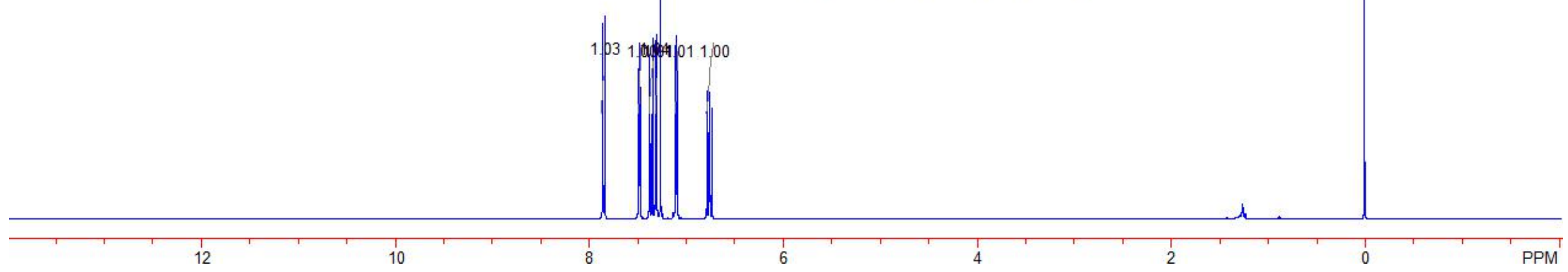




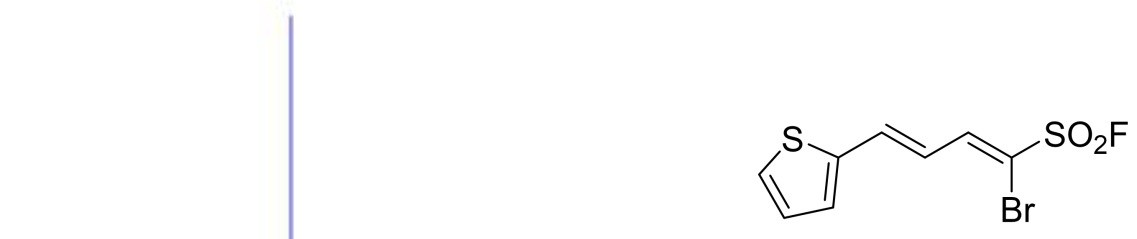

3sa

${ }^{19} \mathrm{~F}$ NMR $\left(471 \mathrm{MHz}, \mathrm{CDCl}_{3}\right)$

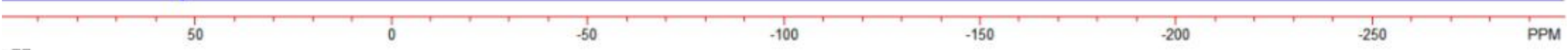



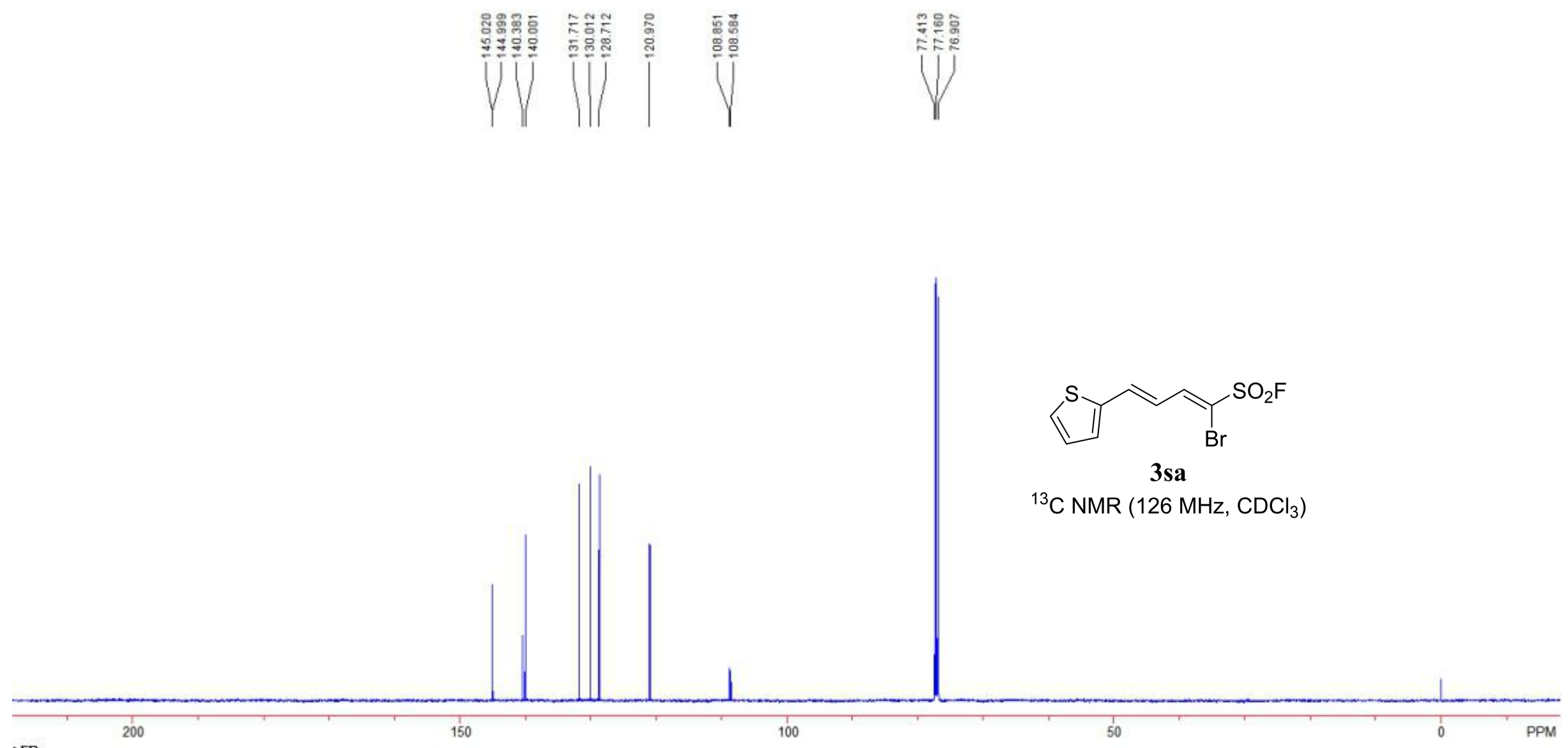

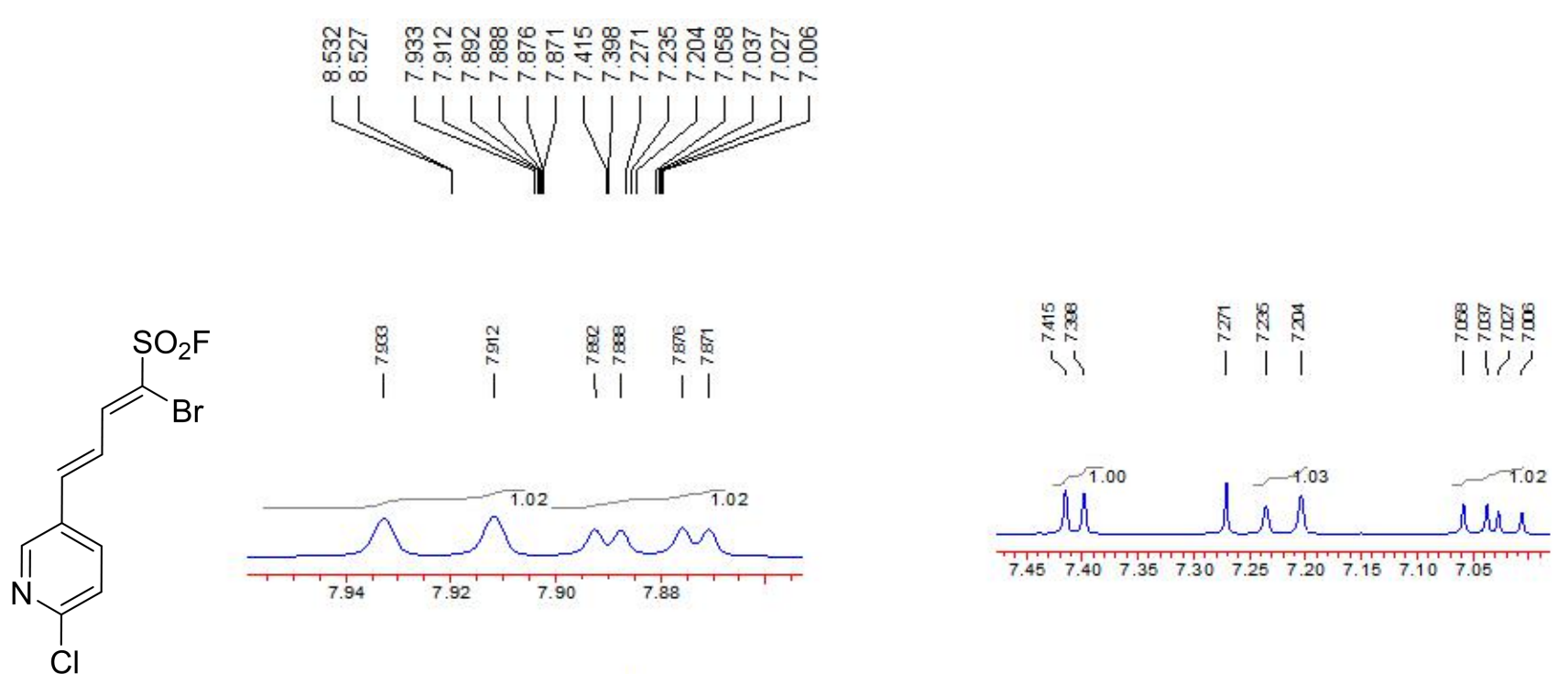

3ta

${ }^{1} \mathrm{H} \mathrm{NMR}\left(500 \mathrm{MHz}, \mathrm{CDCl}_{3}\right)$

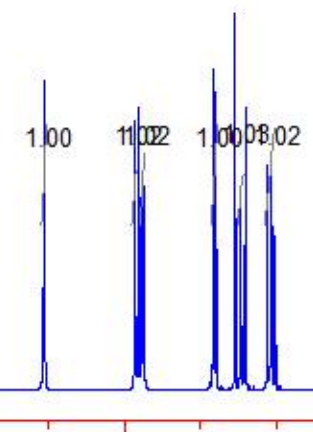

10

8

6

PPM 


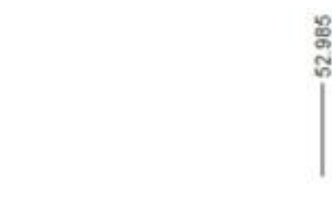

స్요

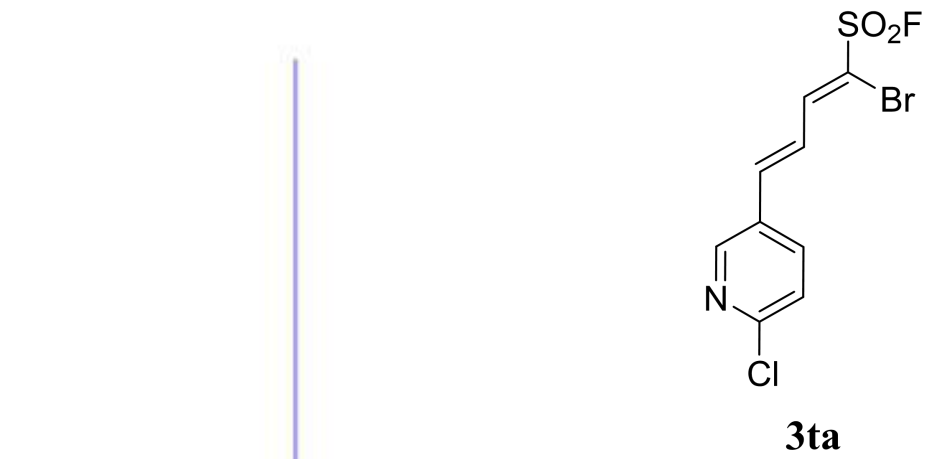

${ }^{19} \mathrm{~F}$ NMR $\left(471 \mathrm{MHz}, \mathrm{CDCl}_{3}\right)$

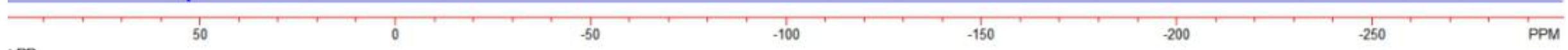

s no 


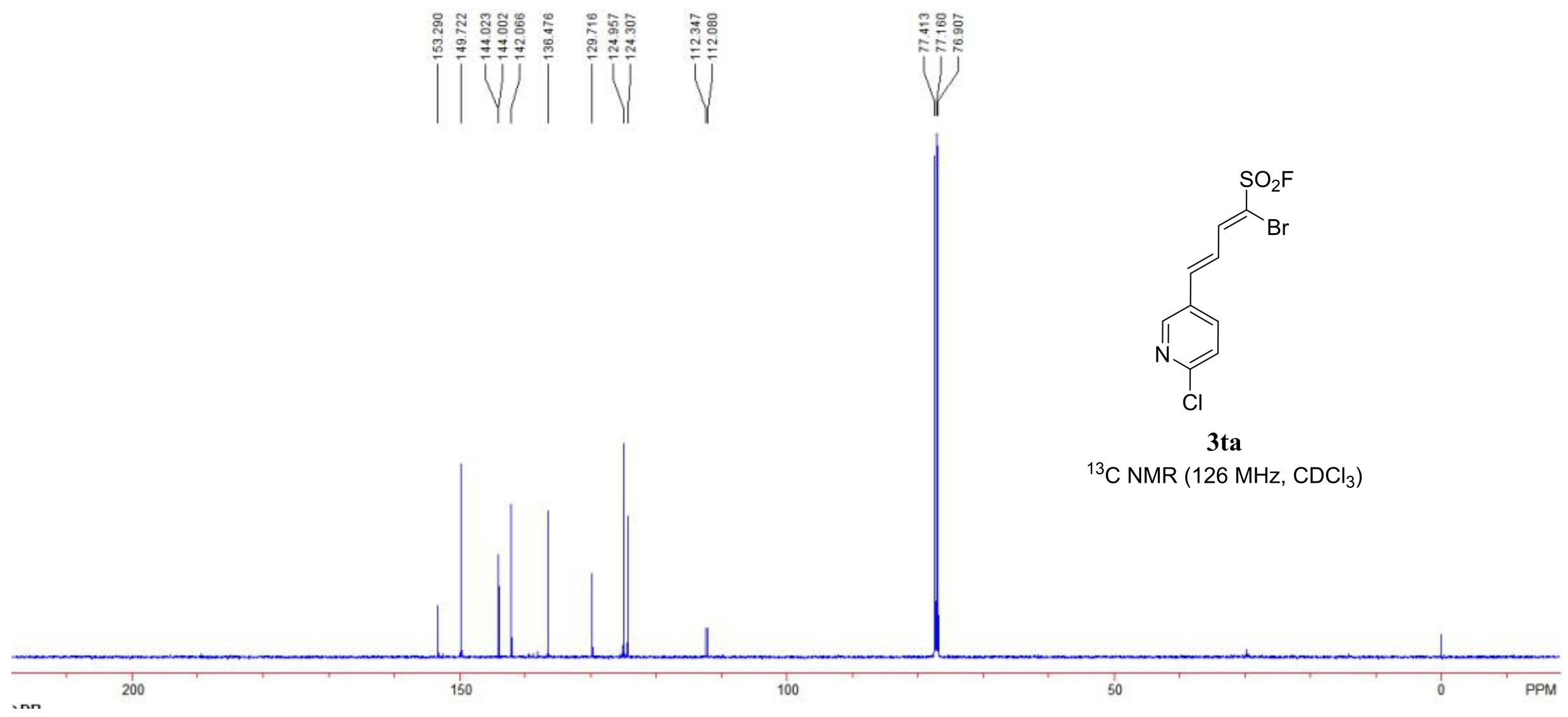




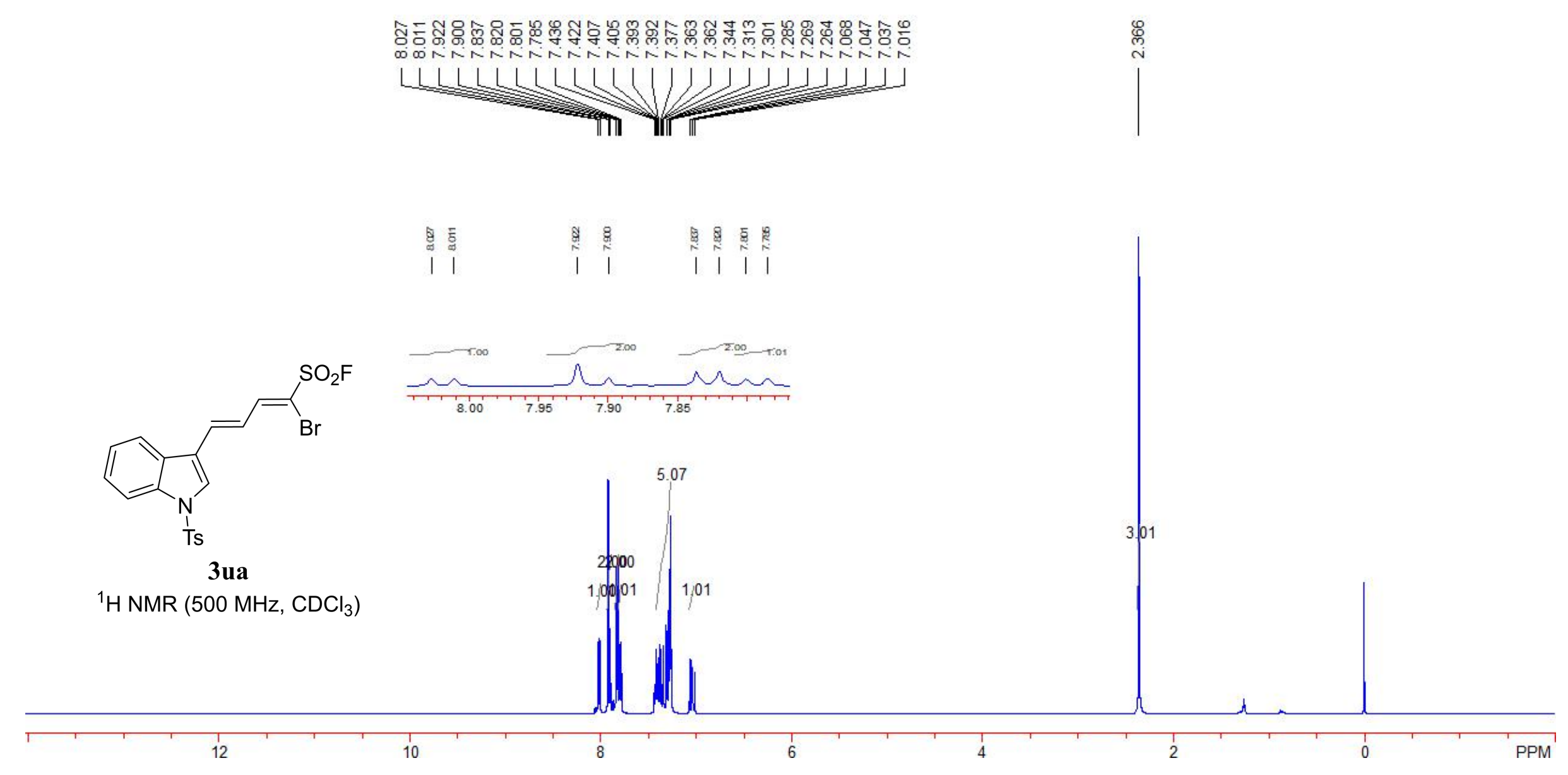




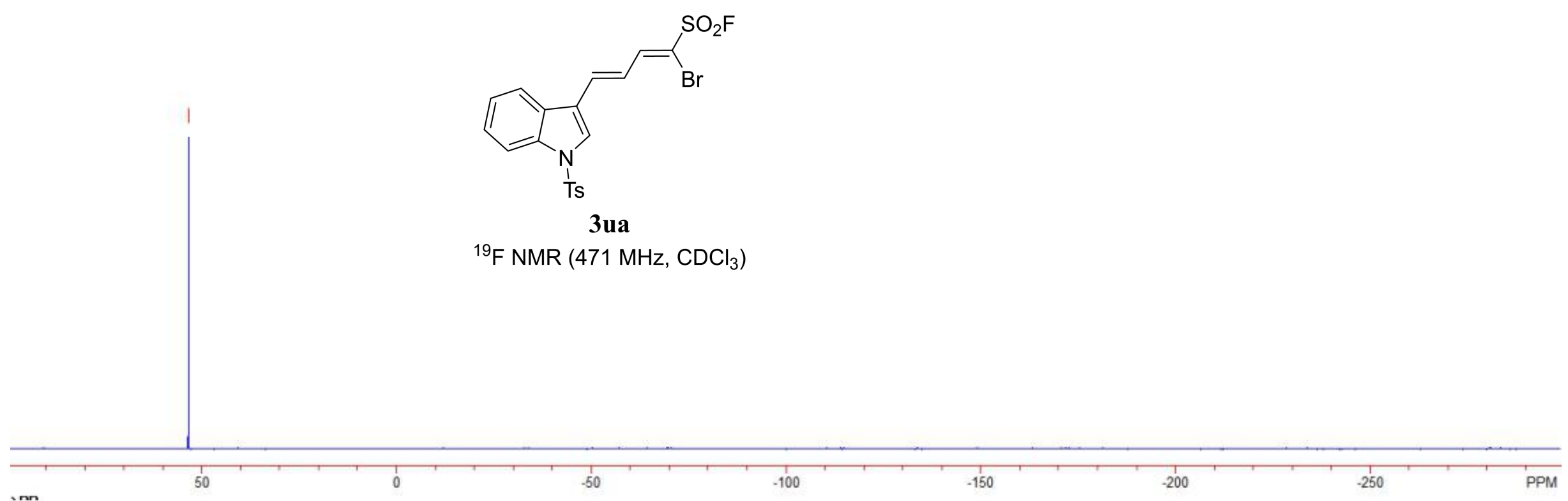



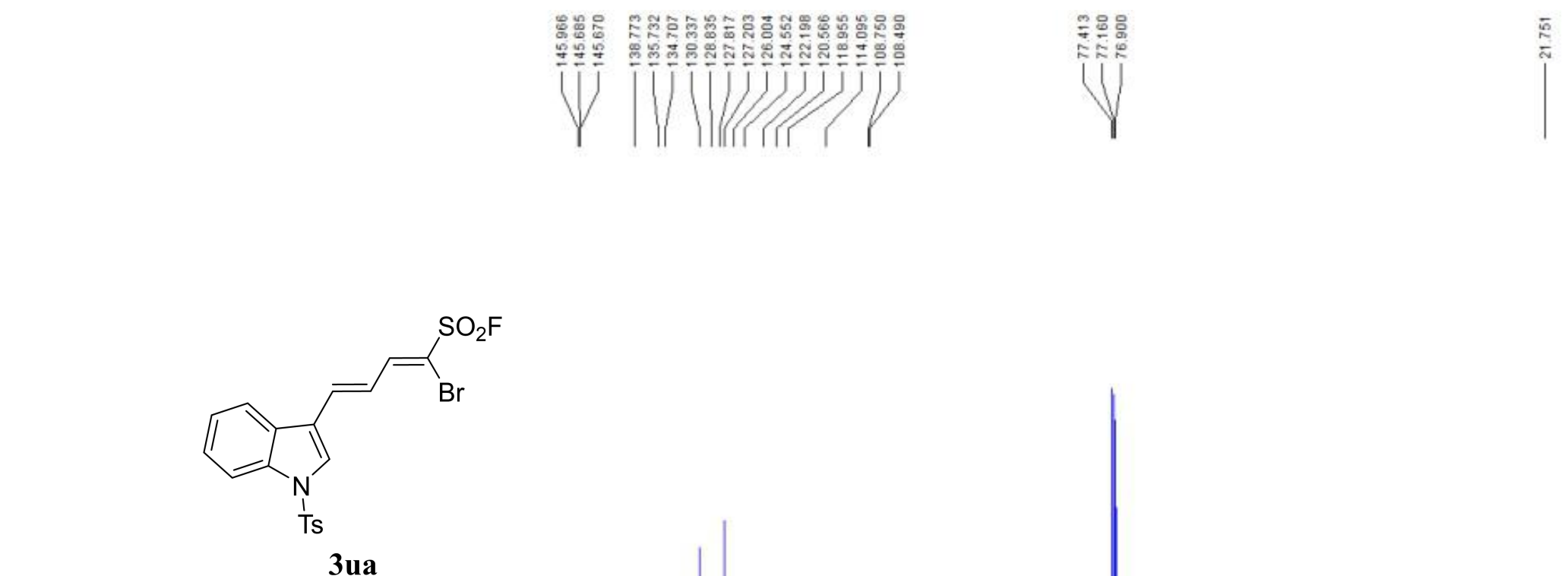

${ }^{13} \mathrm{C}$ NMR (126 MHz, $\mathrm{CDCl}_{3}$ )

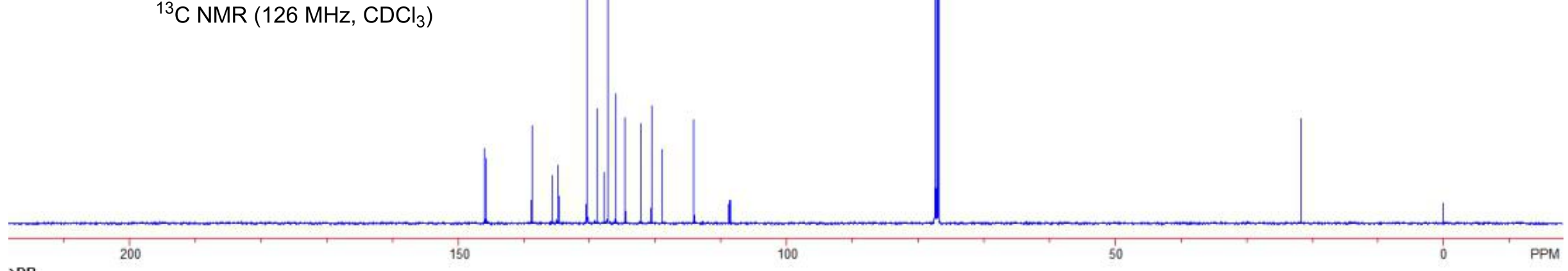



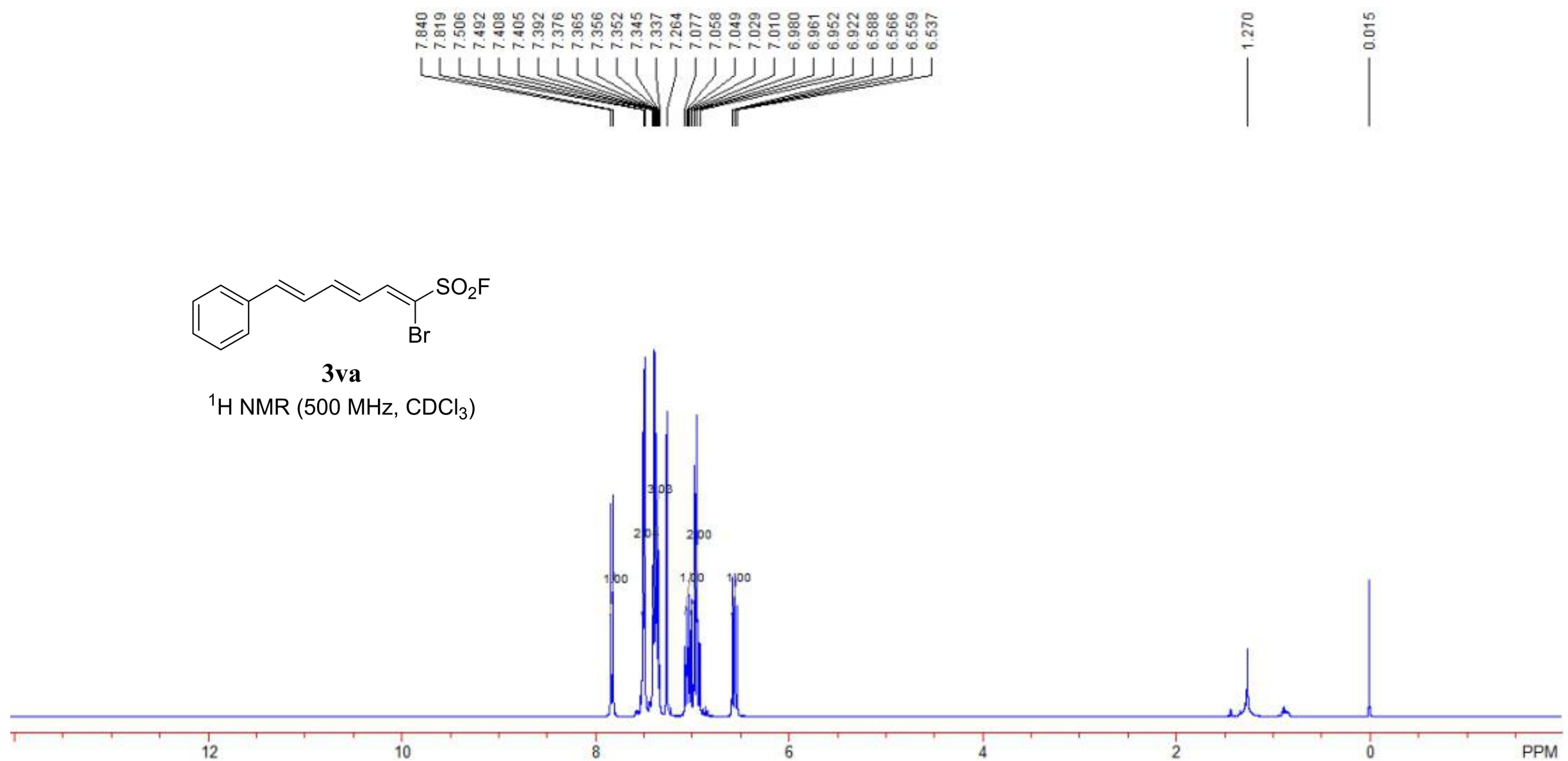


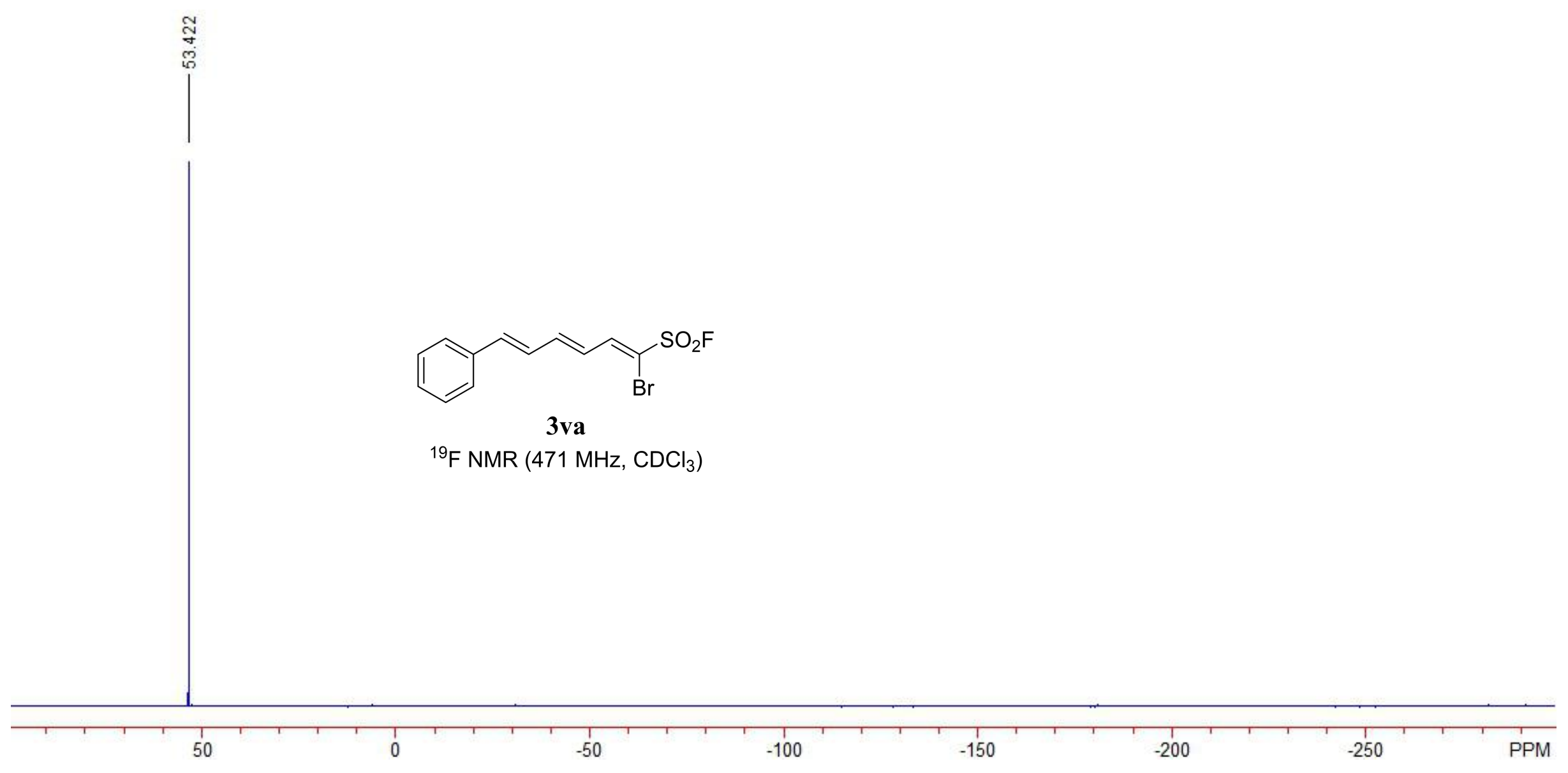




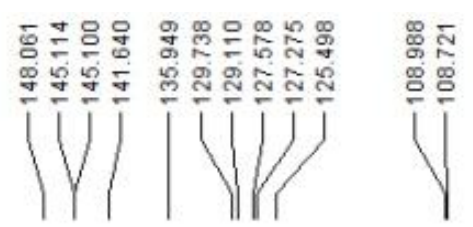

궁으.

KE.

V

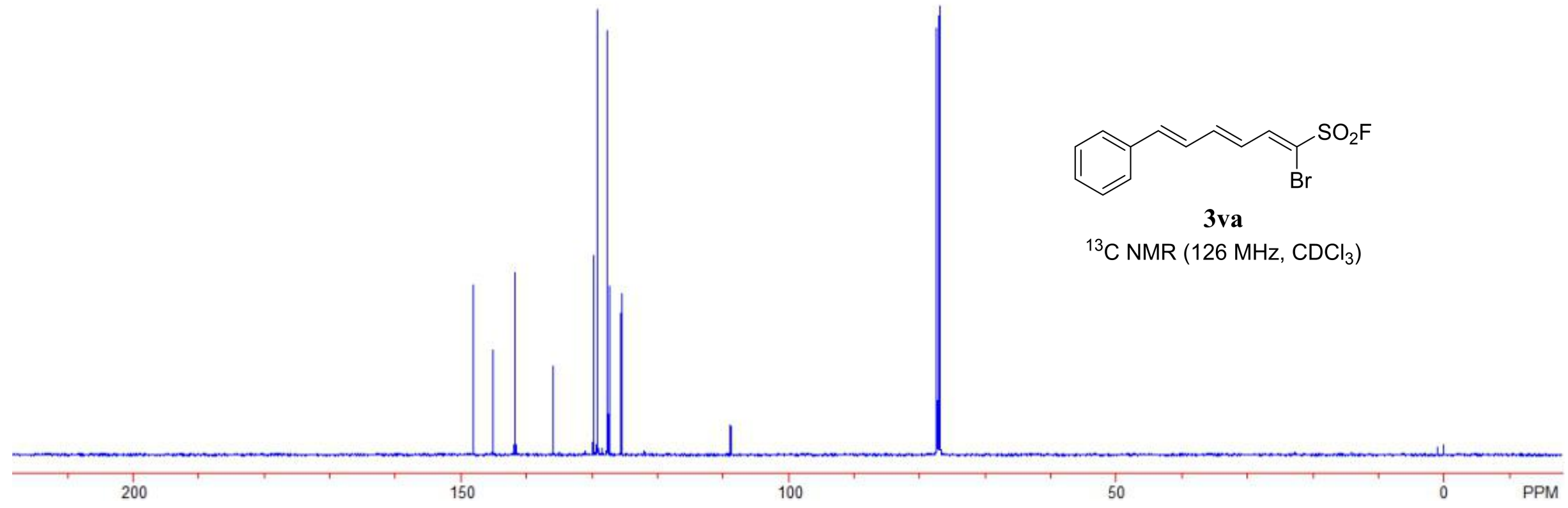




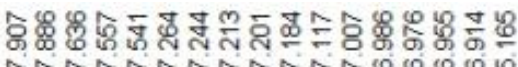

WW
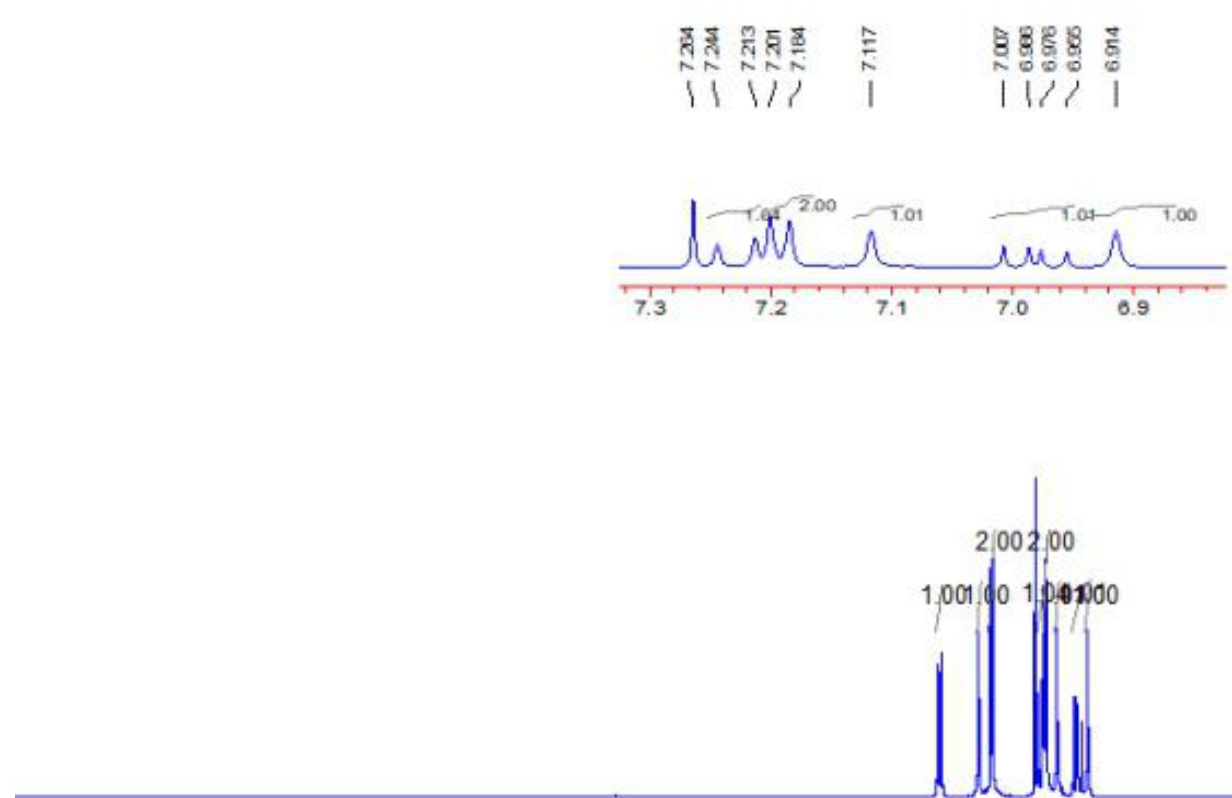

10

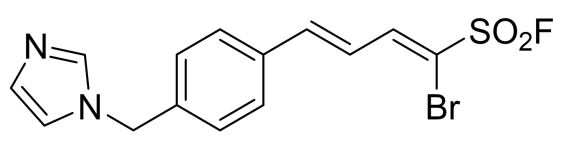

3 wa

${ }^{1} \mathrm{H}$ NMR $\left(500 \mathrm{MHz}, \mathrm{CDCl}_{3}\right)$ 
|

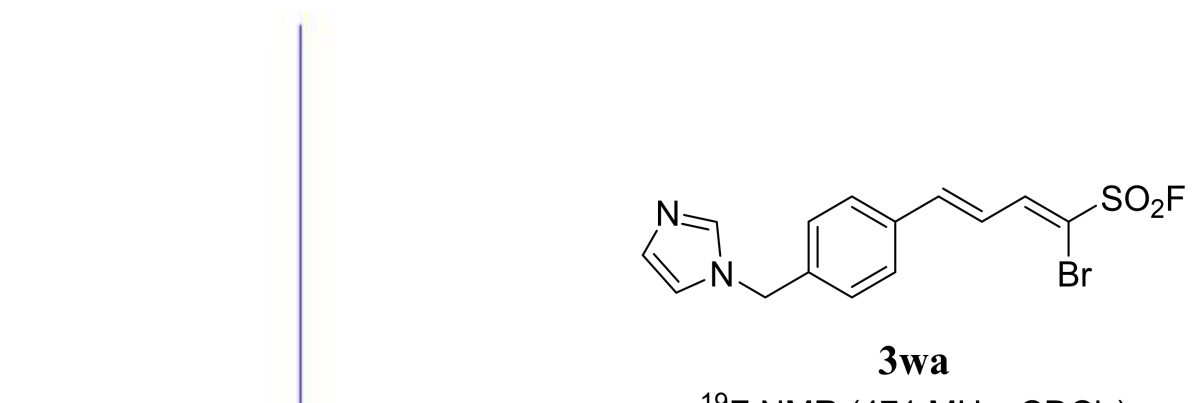

${ }^{19} \mathrm{~F} \mathrm{NMR}\left(471 \mathrm{MHz}, \mathrm{CDCl}_{3}\right)$ 

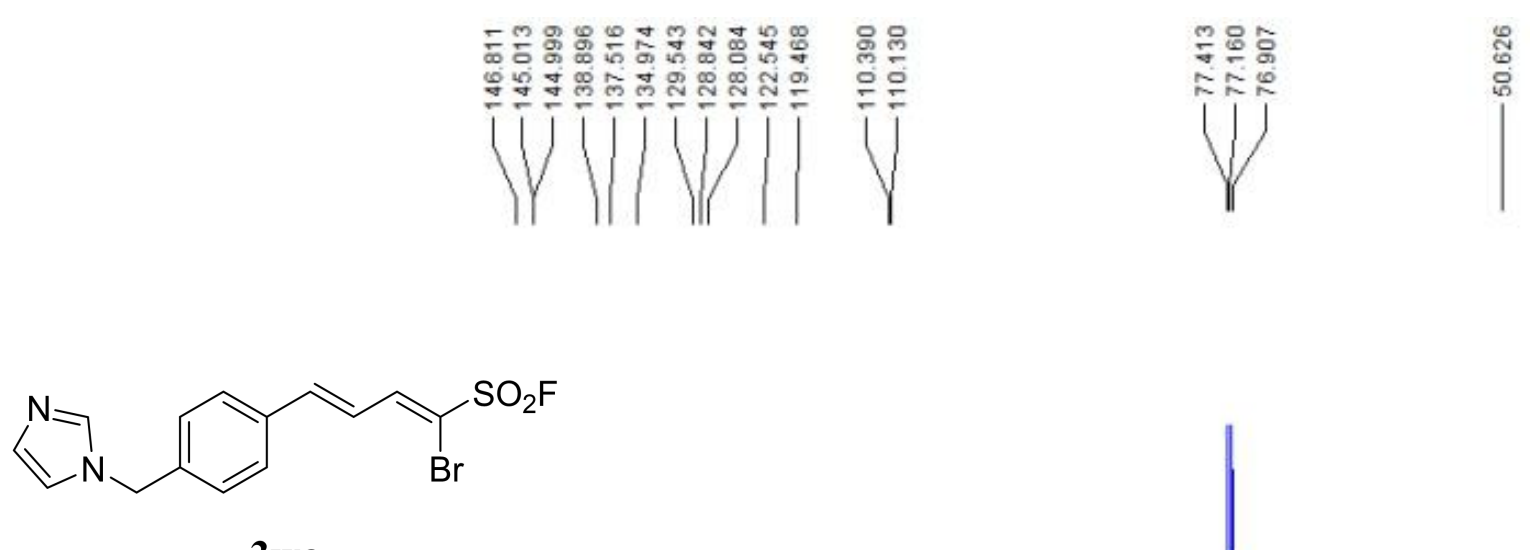

3wa

$\left.{ }^{13} \mathrm{C} \mathrm{NMR} \mathrm{(126} \mathrm{MHz,} \mathrm{CDCl}_{3}\right)$

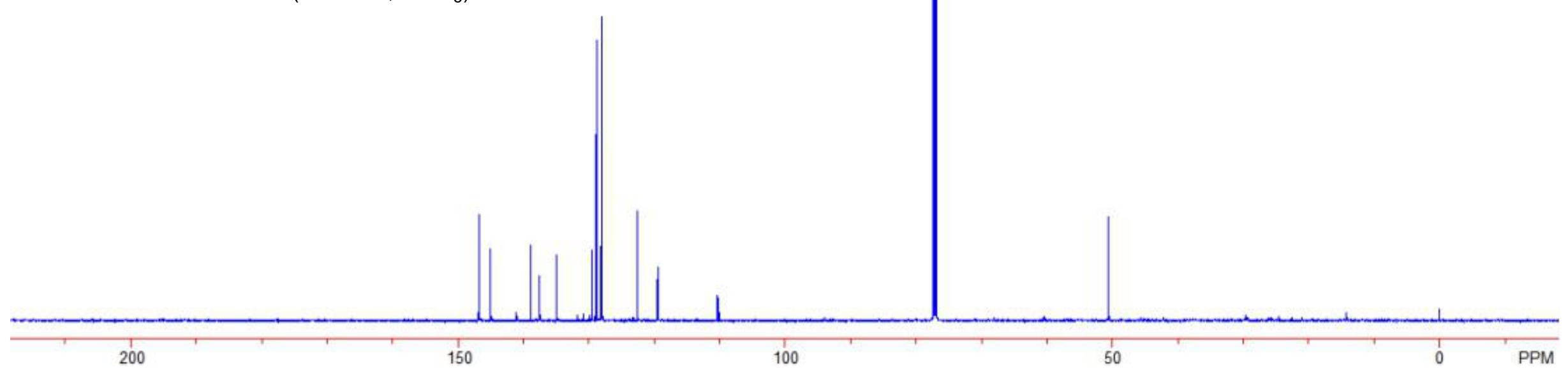



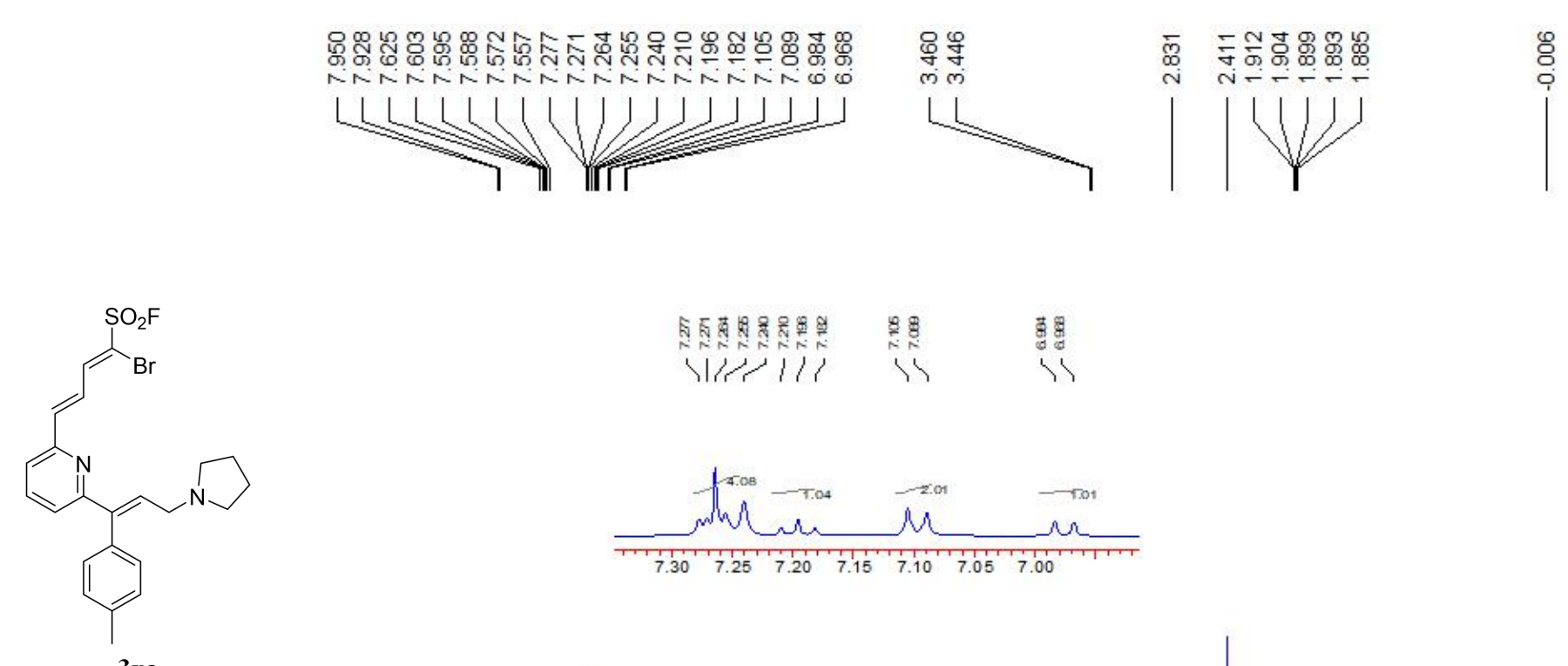

${ }^{1} \mathrm{H}$ NMR $\left(500 \mathrm{MHz}, \mathrm{CDCl}_{3}\right)$

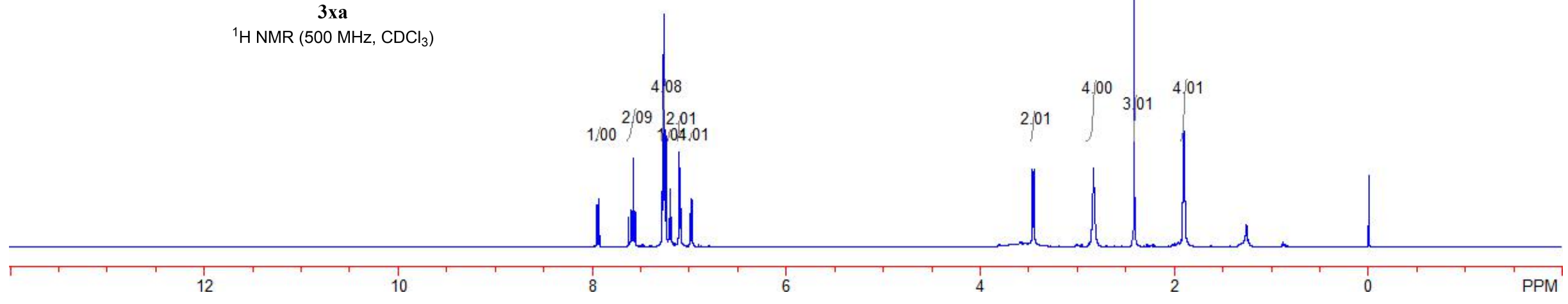




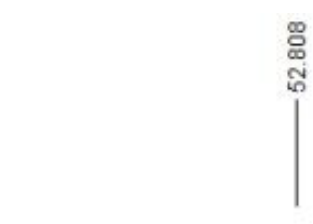

|

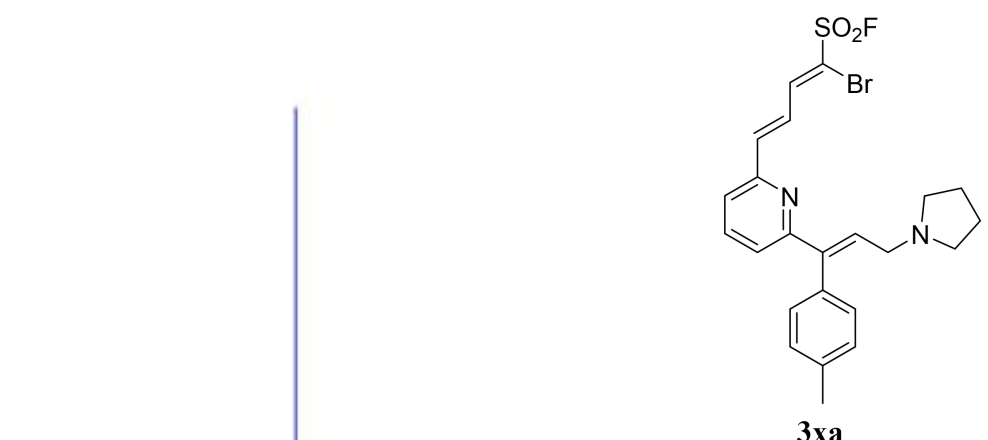

${ }^{19} \mathrm{~F} \mathrm{NMR}\left(471 \mathrm{MHz}, \mathrm{CDCl}_{3}\right)$

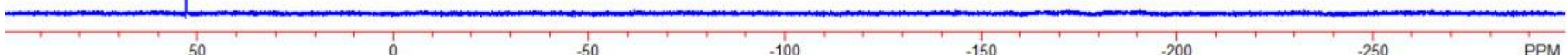



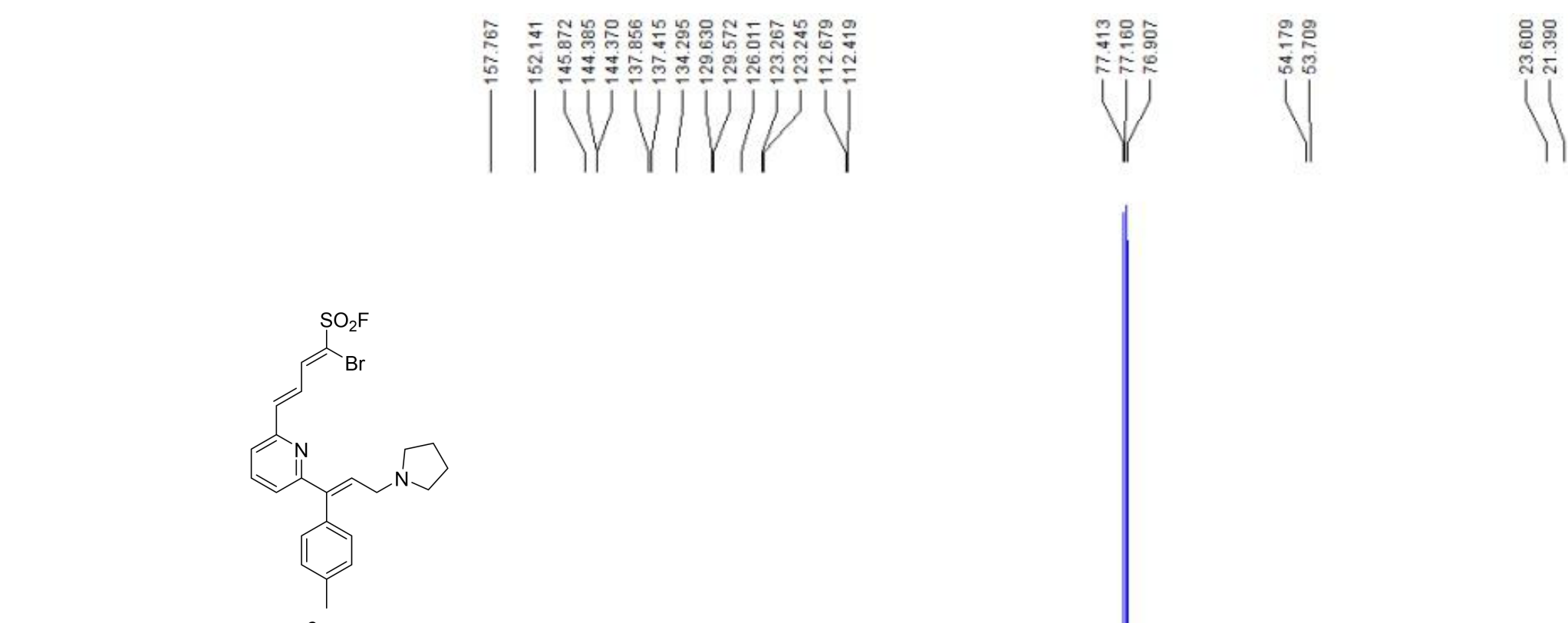

${ }^{13} \mathrm{C}$ NMR $\left(126 \mathrm{MHz}, \mathrm{CDCl}_{3}\right)$

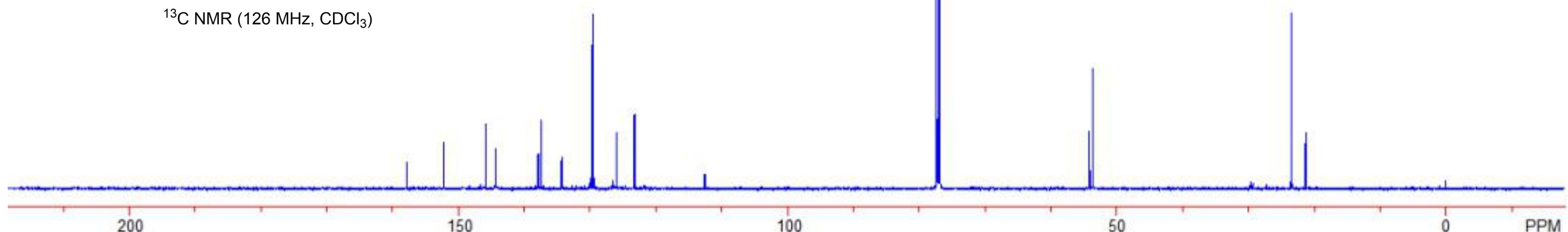




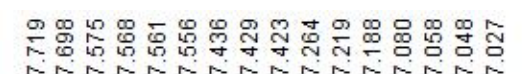

uiviujjjjjjJ

Nitir

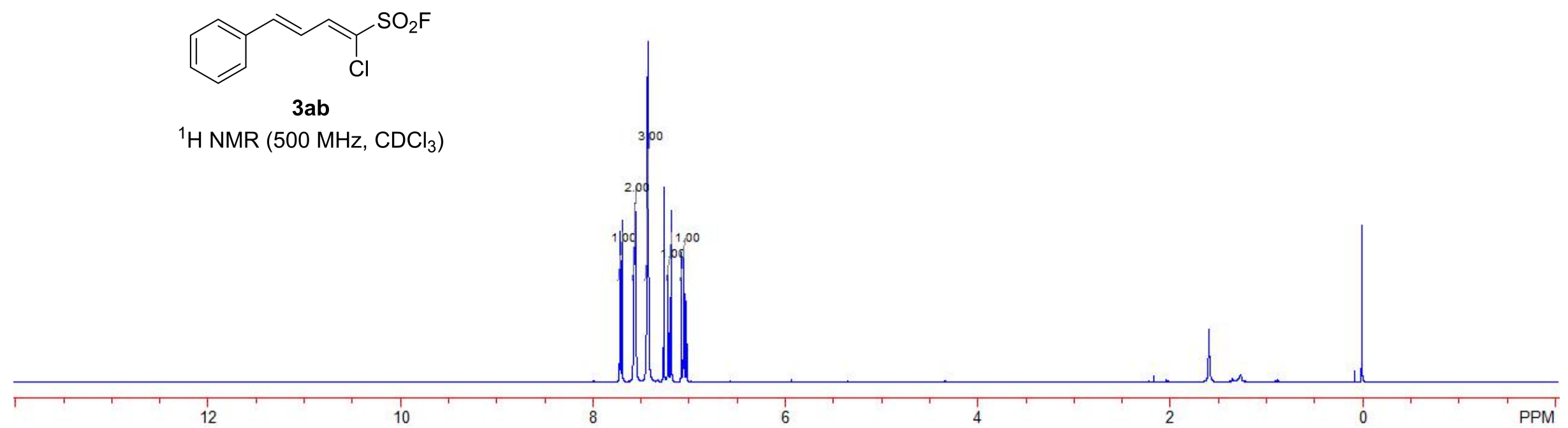




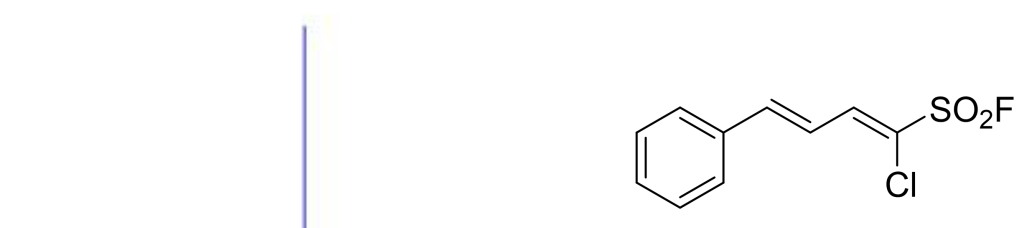

$3 a b$

${ }^{19} \mathrm{~F}$ NMR $\left(471 \mathrm{MHz}, \mathrm{CDCl}_{3}\right)$ 


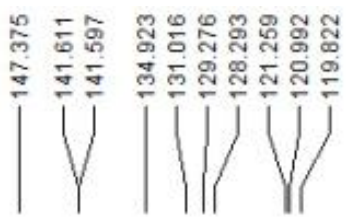

욱훙

주

W

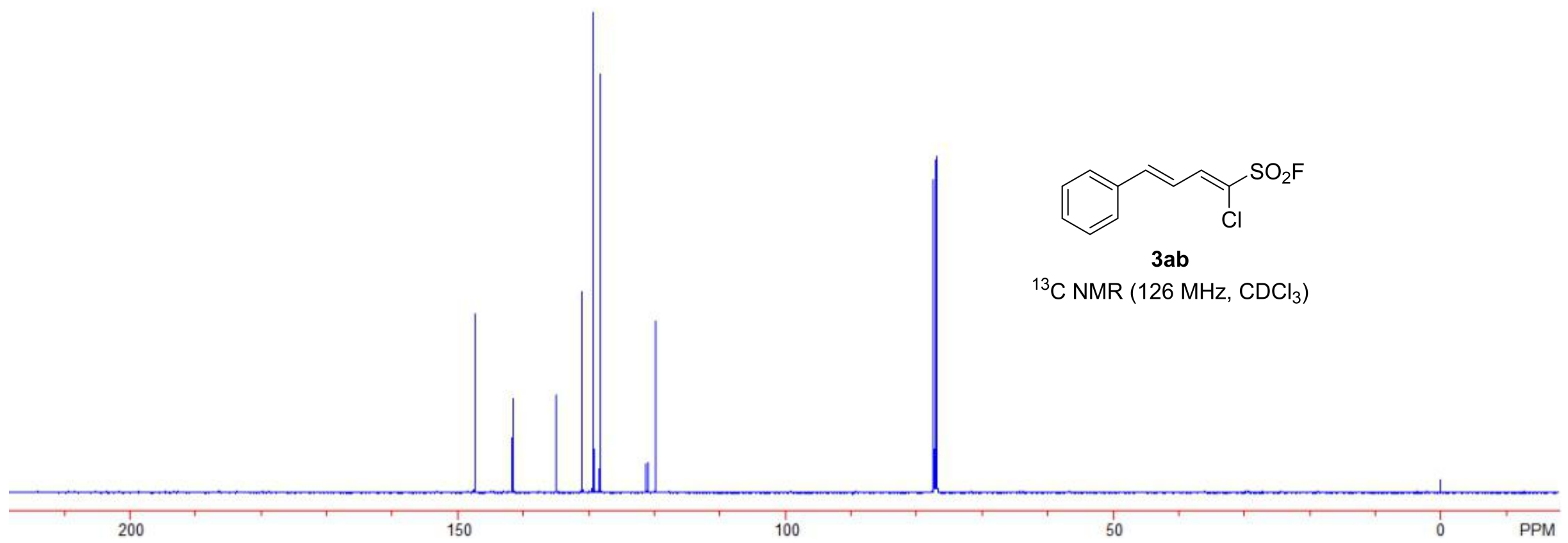




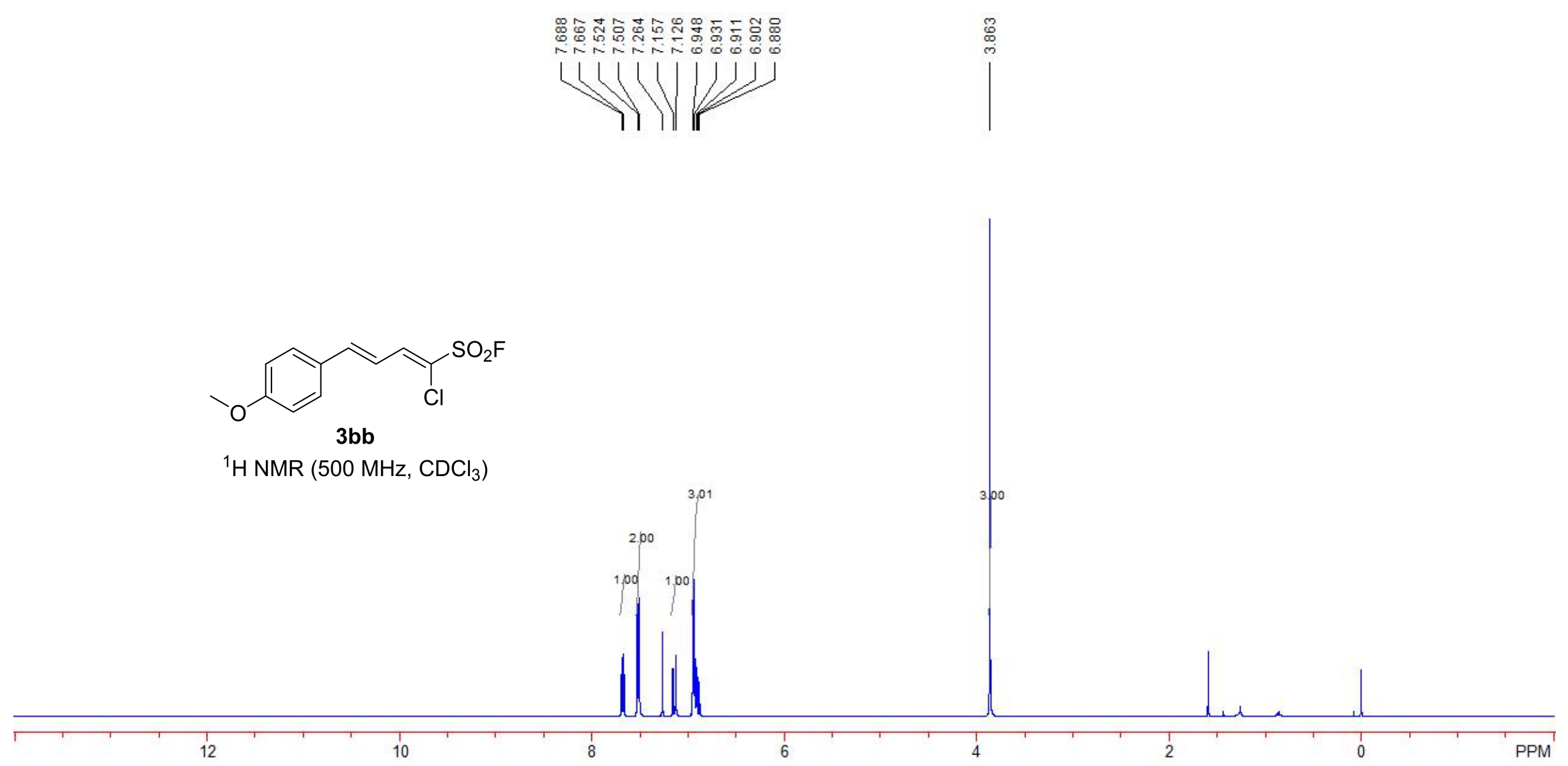


|

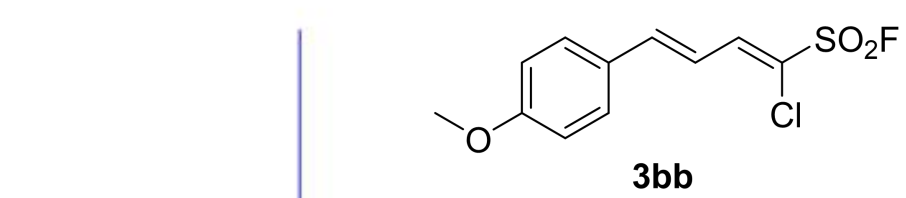

${ }^{19} \mathrm{~F} \mathrm{NMR}\left(471 \mathrm{MHz}, \mathrm{CDCl}_{3}\right)$ 

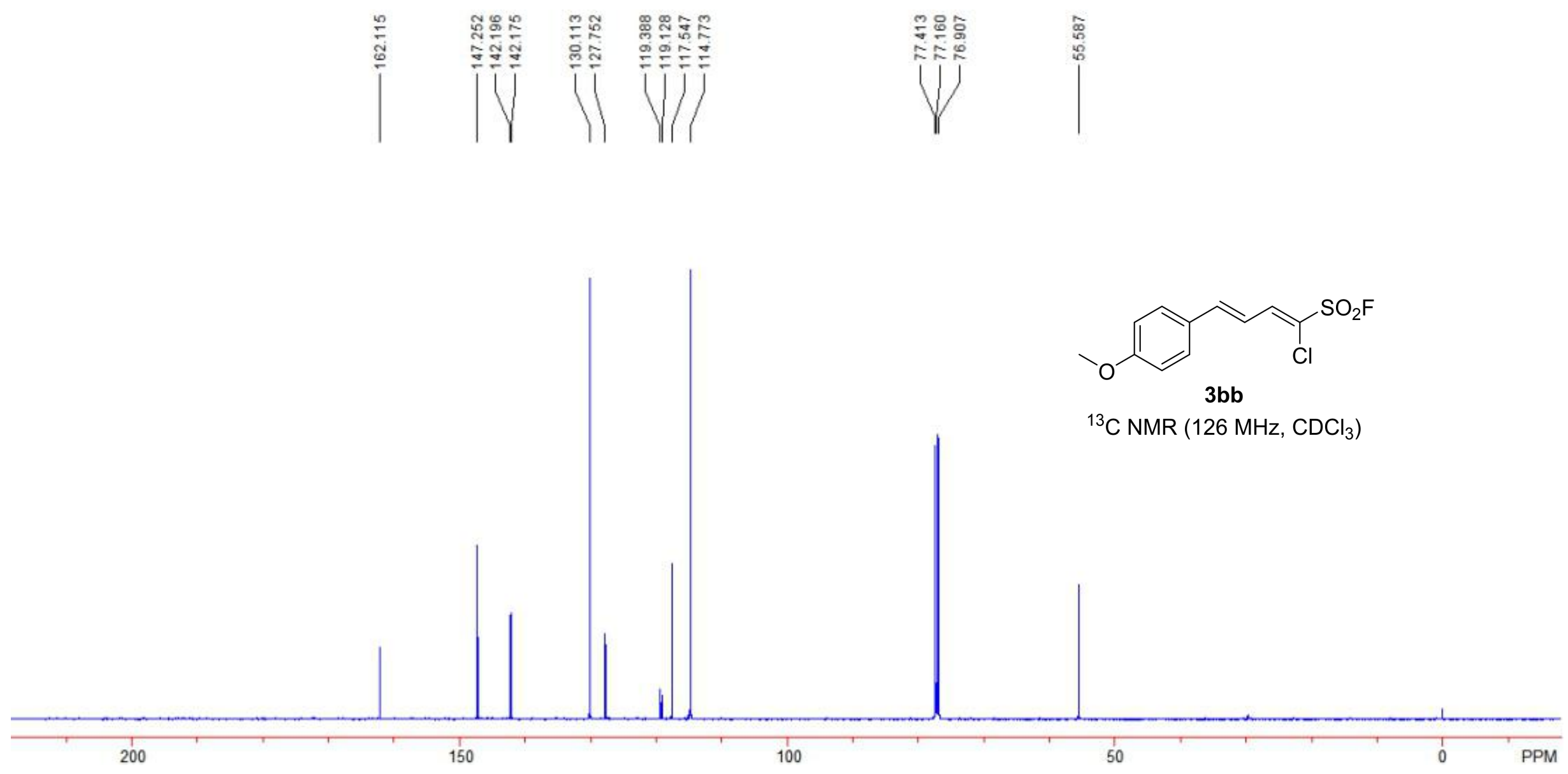


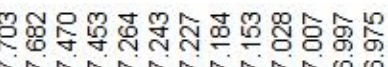

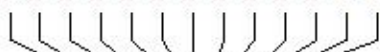

$71 /$ r
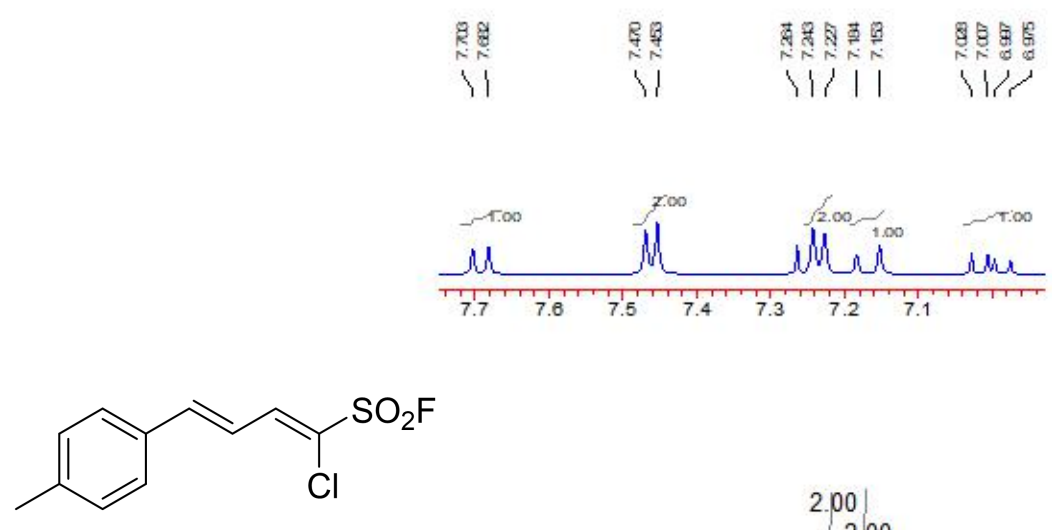

$3 \mathrm{cb}$

${ }^{1} \mathrm{H} \mathrm{NMR}\left(500 \mathrm{MHz}, \mathrm{CDCl}_{3}\right)$

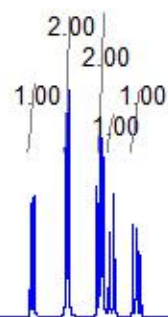

PPM 


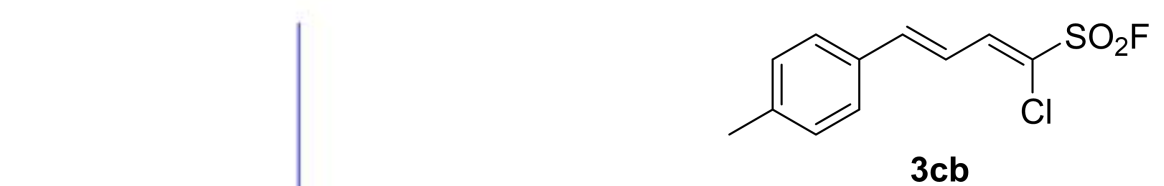

${ }^{19} \mathrm{~F}$ NMR $\left(471 \mathrm{MHz}, \mathrm{CDCl}_{3}\right)$ 

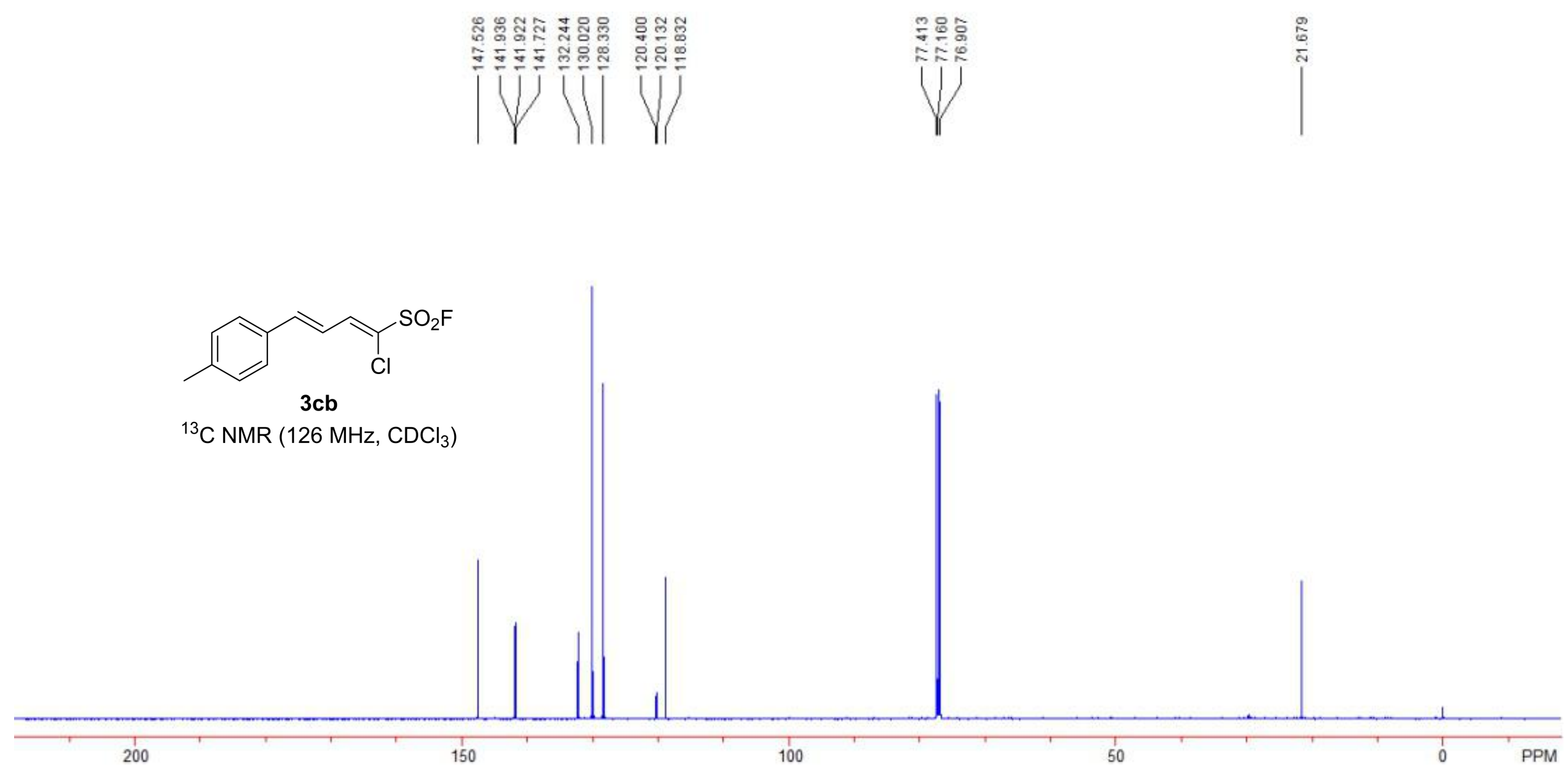


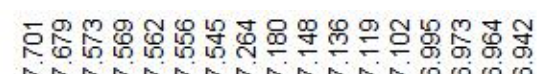

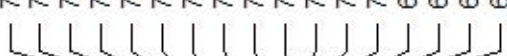

का 1
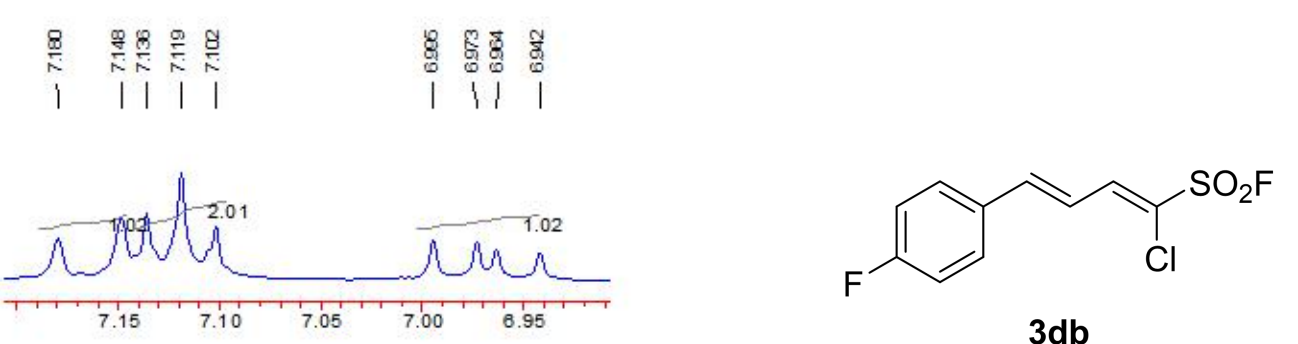

$3 \mathrm{db}$

${ }^{1} \mathrm{H}$ NMR $\left(500 \mathrm{MHz}, \mathrm{CDCl}_{3}\right)$

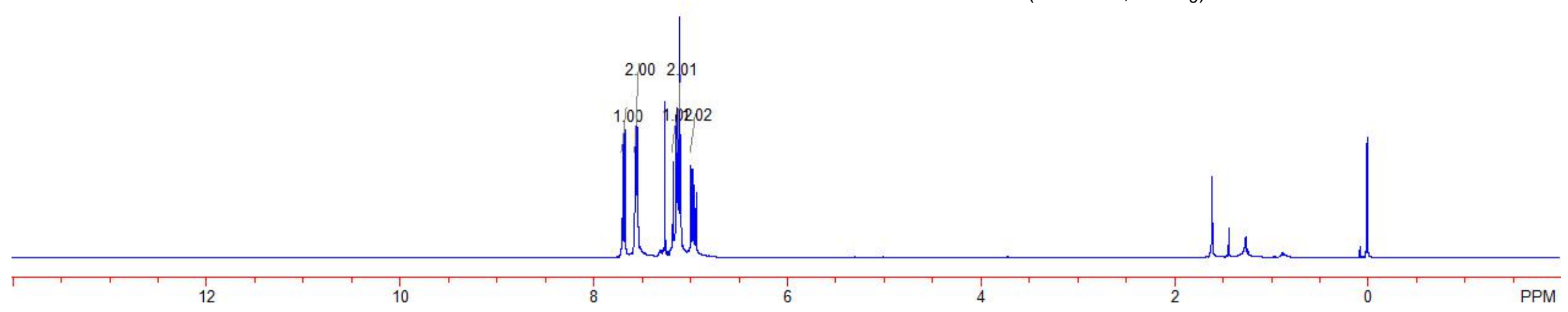



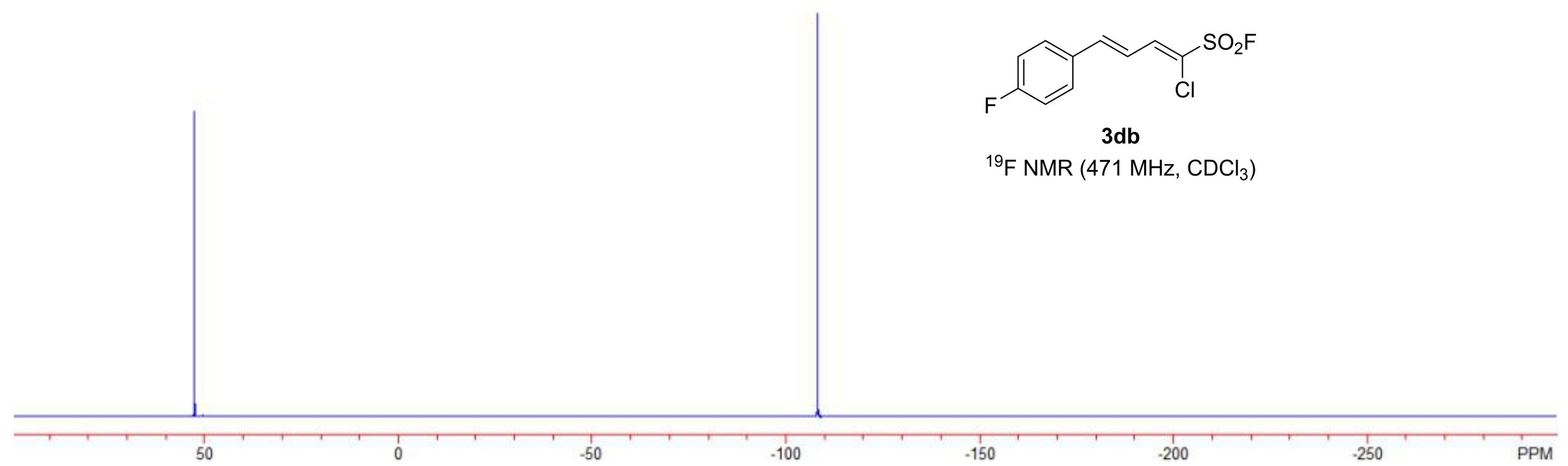


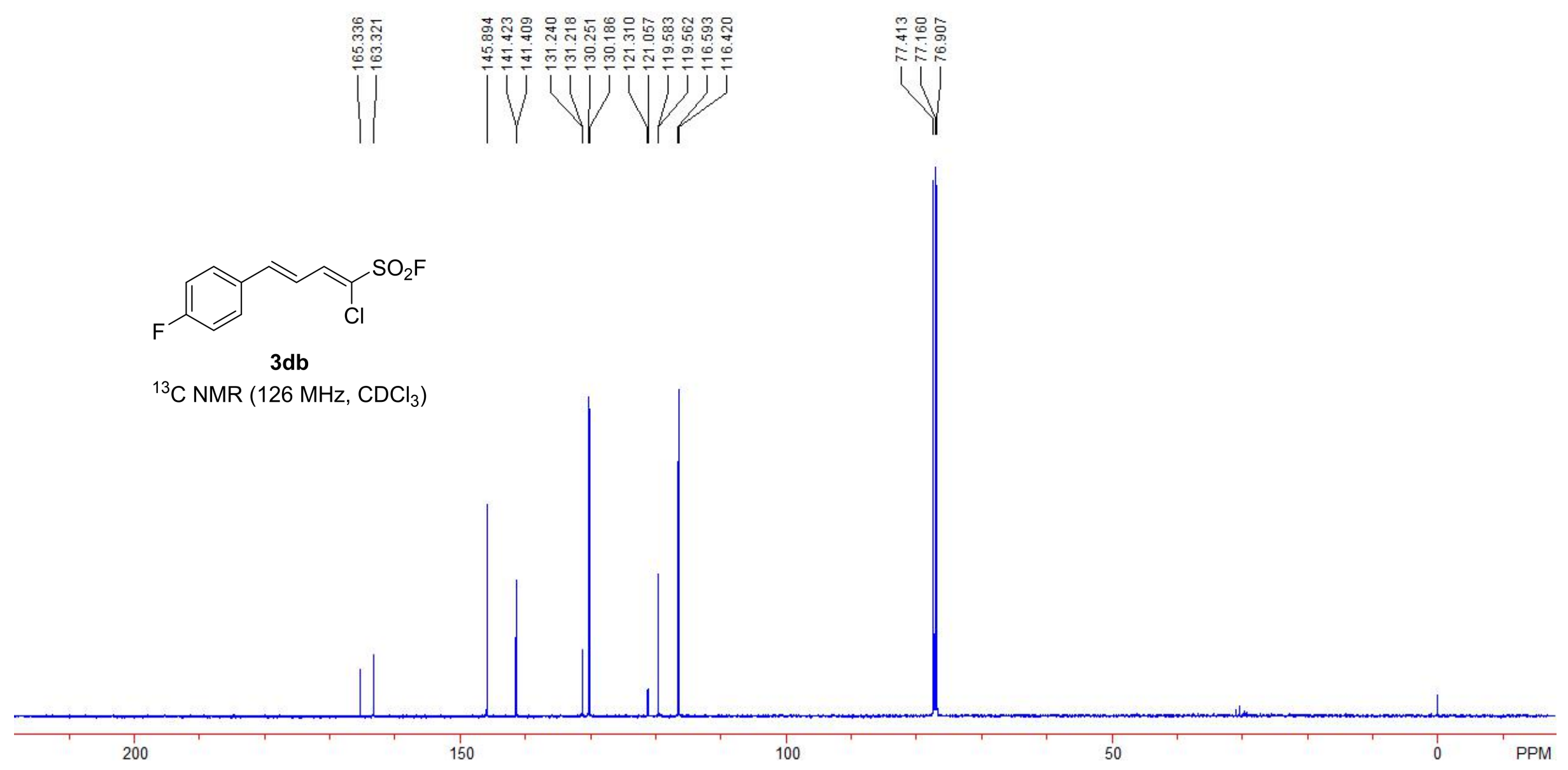




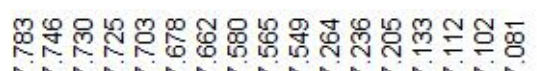

$\lfloor\mid\lfloor|1| 1|1| 1 \mid\rfloor\rfloor\rfloor$
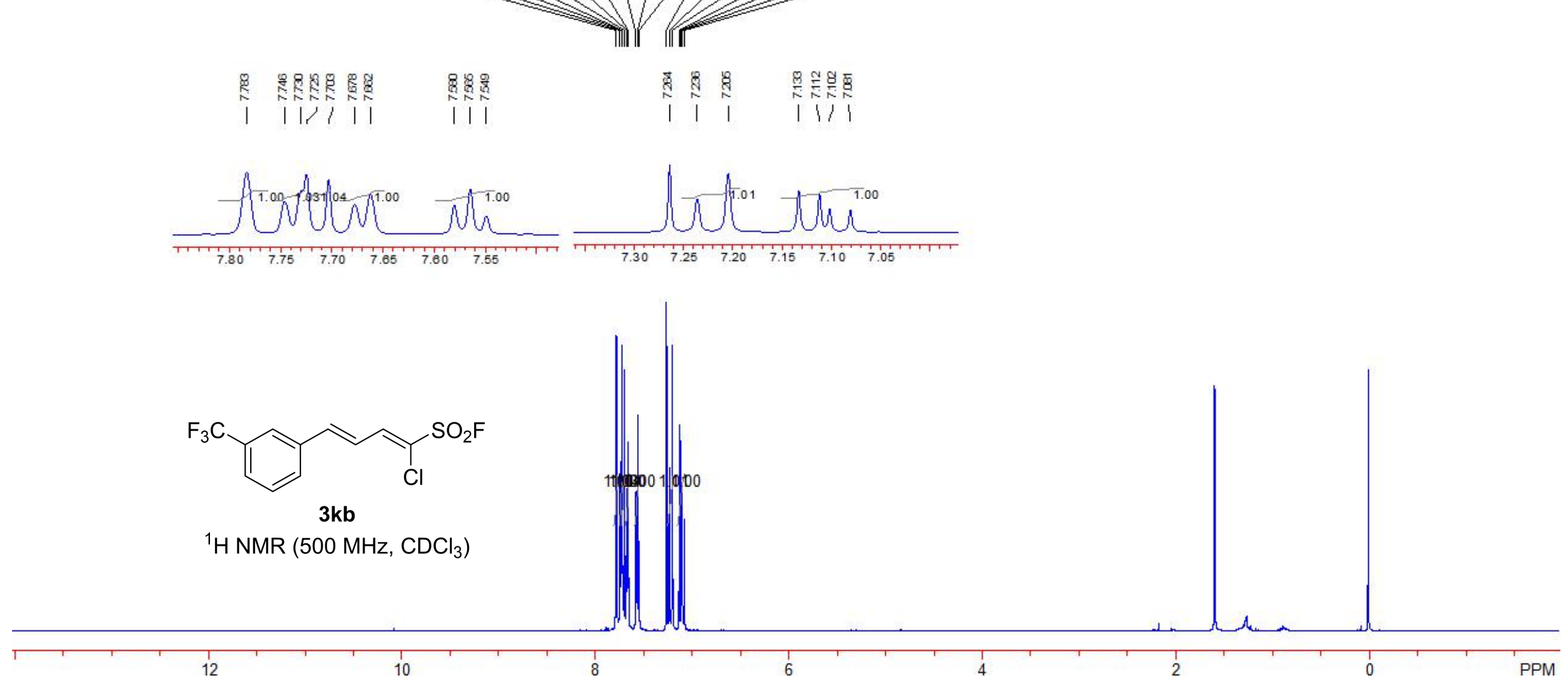

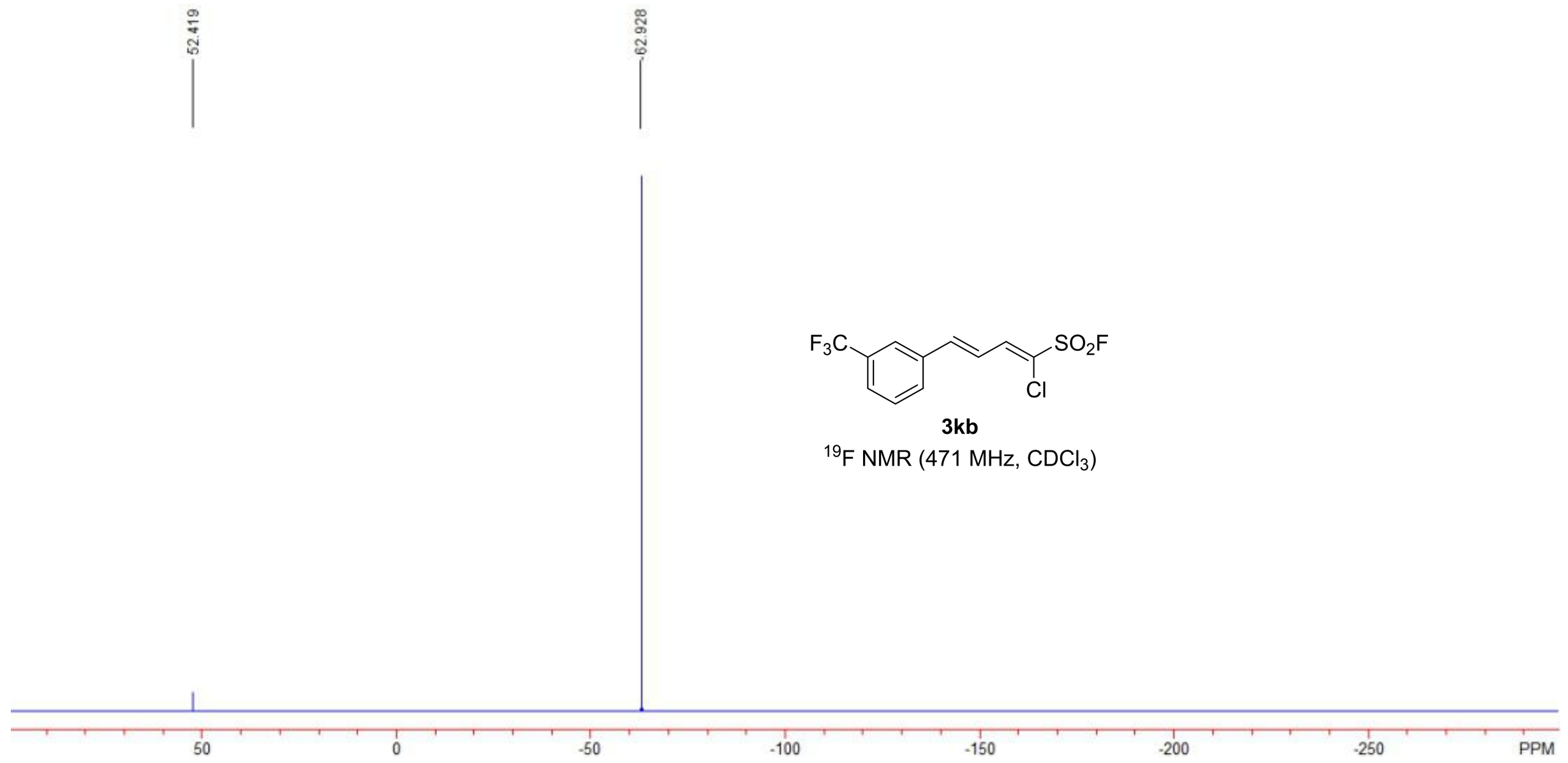

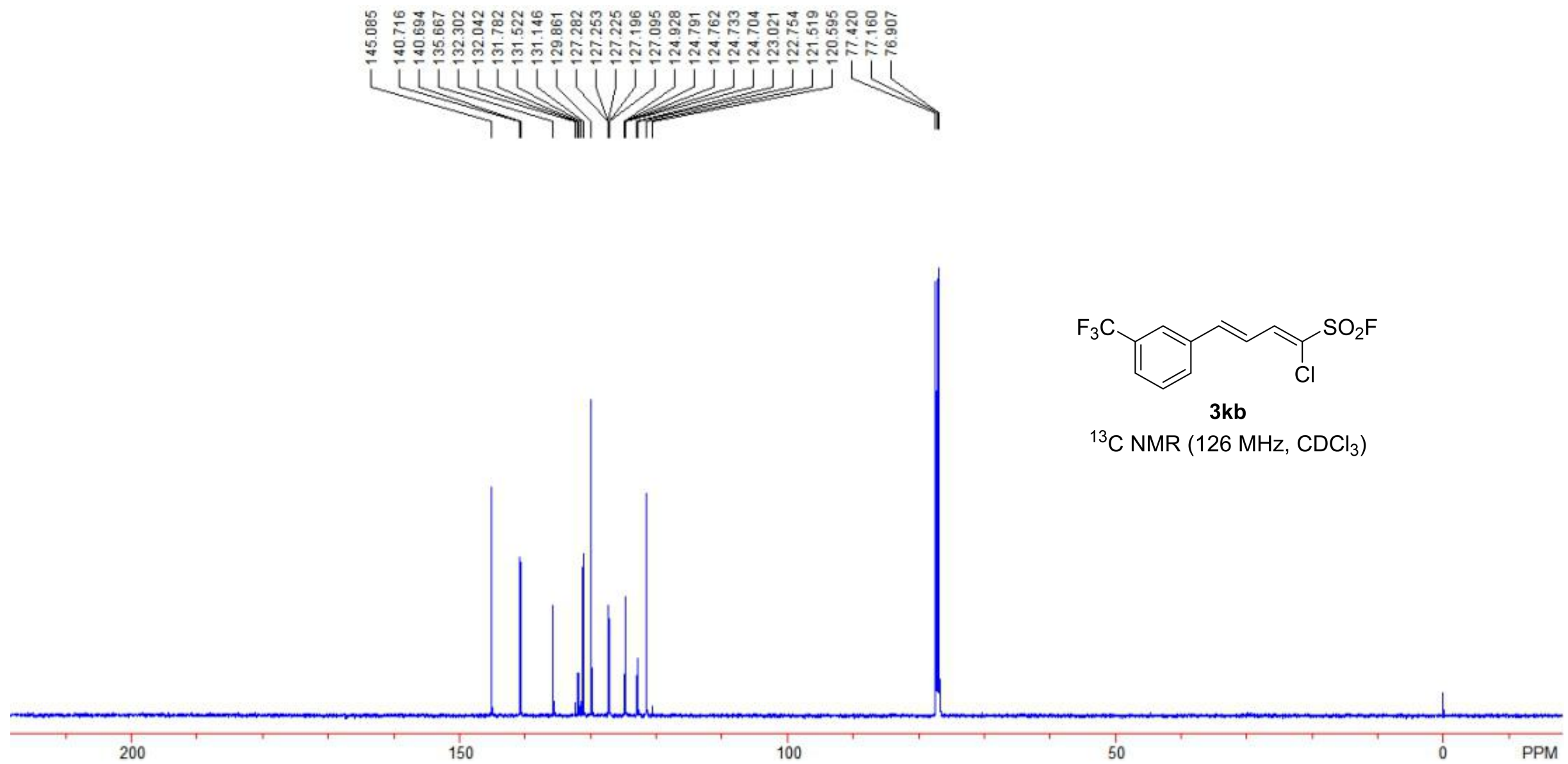

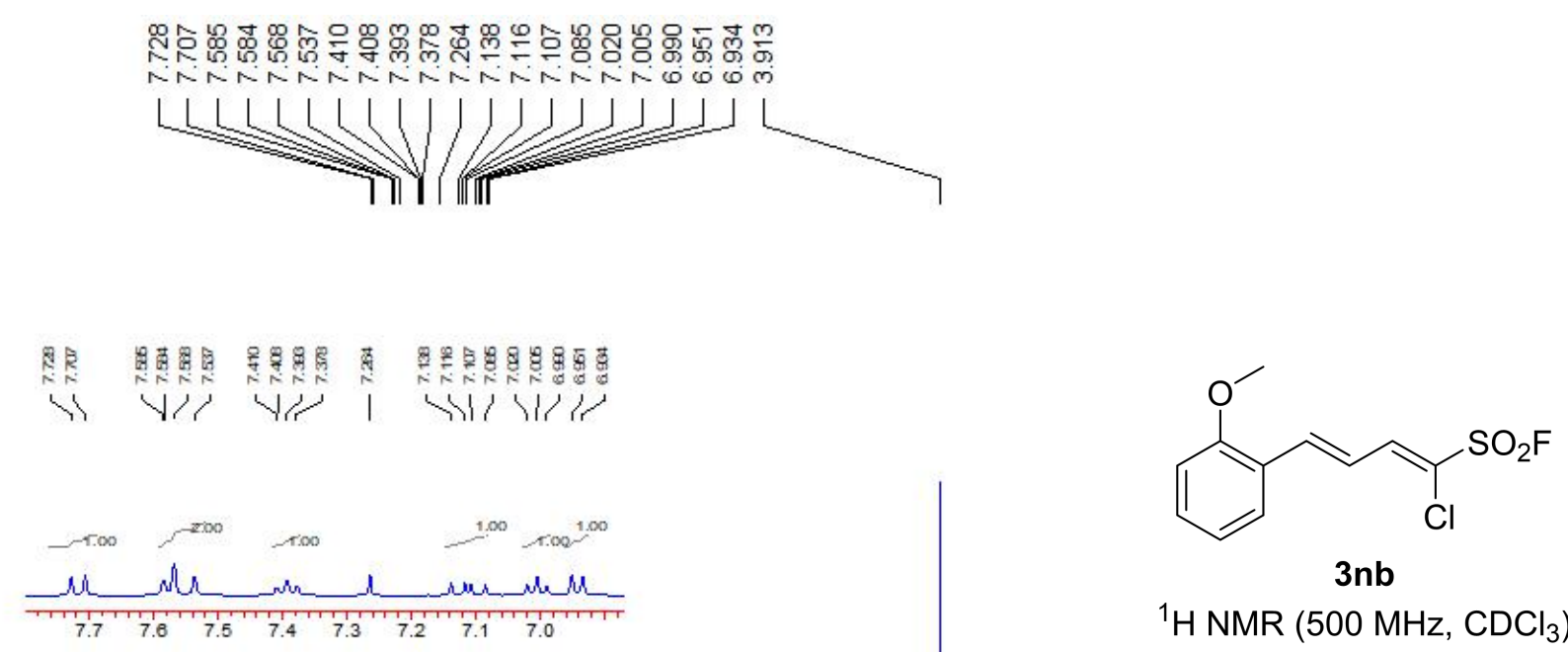

$3 n b$

${ }^{1} \mathrm{H}$ NMR (500 MHz, $\mathrm{CDCl}_{3}$ )

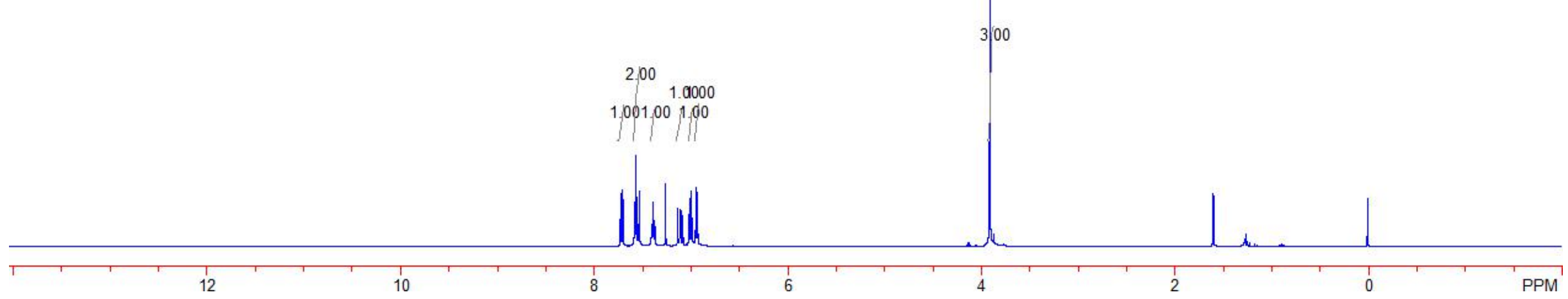


|

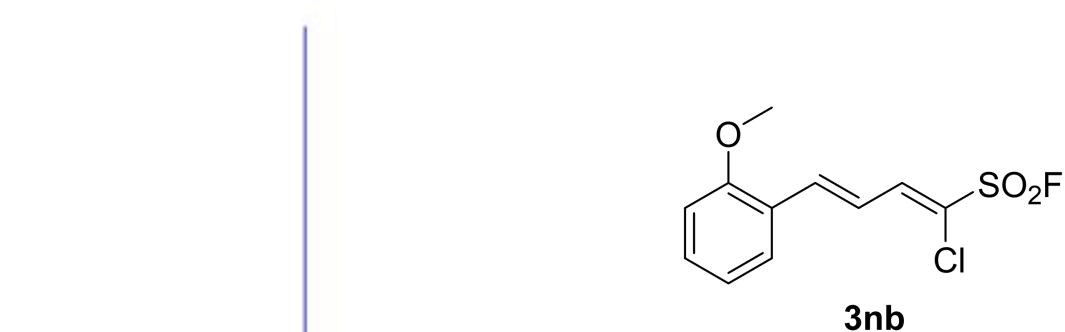

${ }^{19} \mathrm{~F} \mathrm{NMR}\left(471 \mathrm{MHz}, \mathrm{CDCl}_{3}\right)$ 

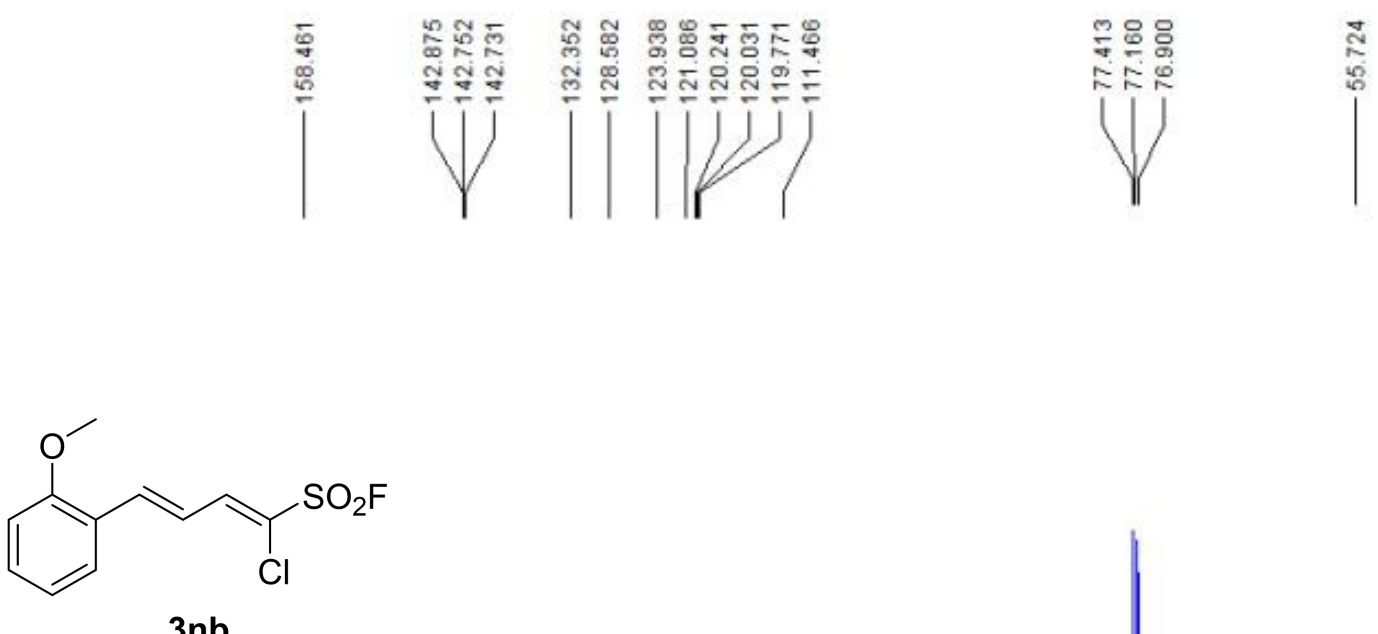

3nb

${ }^{13} \mathrm{C}$ NMR $\left(126 \mathrm{MHz}, \mathrm{CDCl}_{3}\right)$

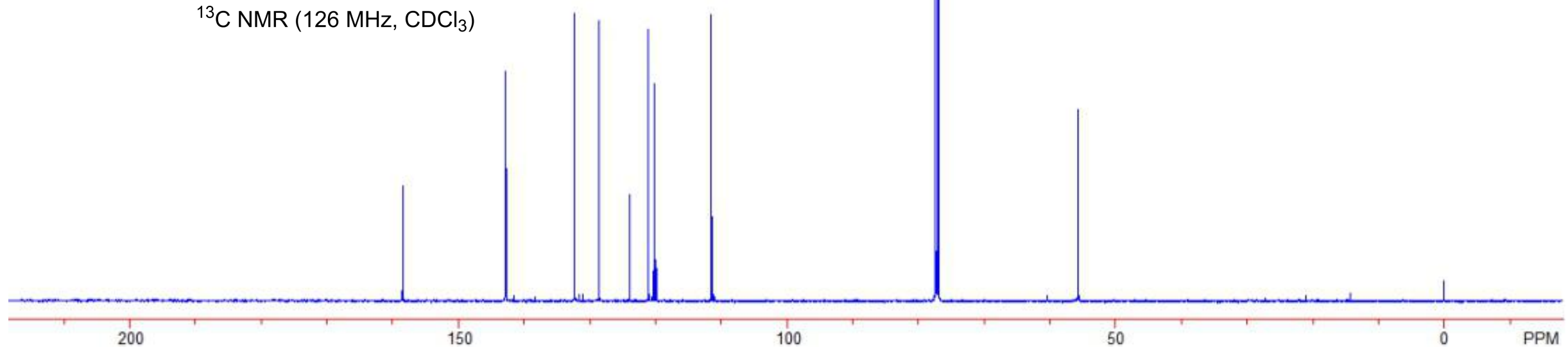



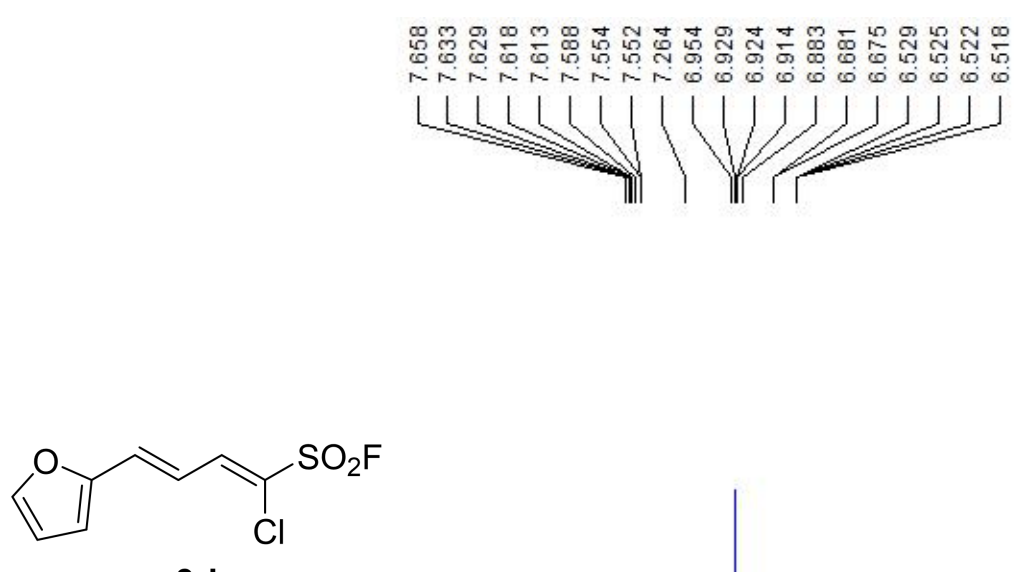

3 rb

${ }^{1} \mathrm{H} \mathrm{NMR}\left(500 \mathrm{MHz}, \mathrm{CDCl}_{3}\right)$

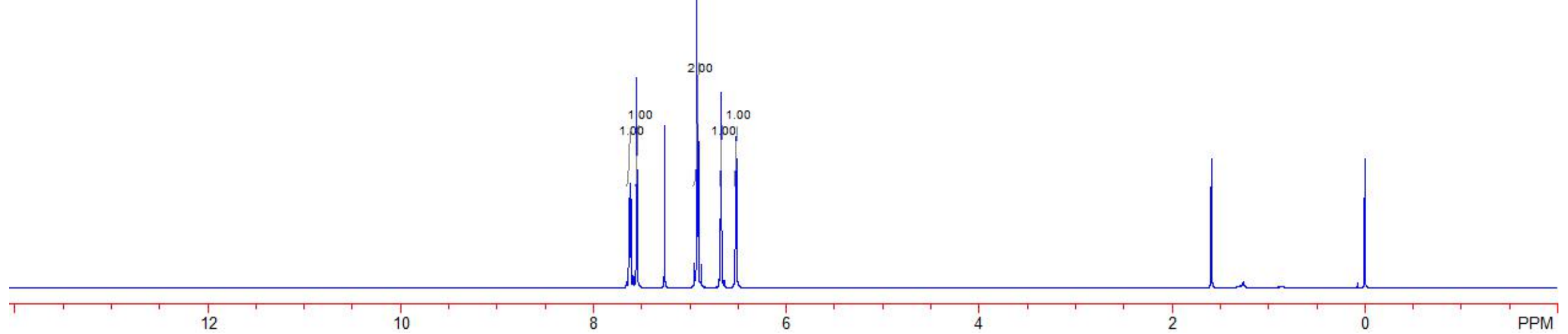




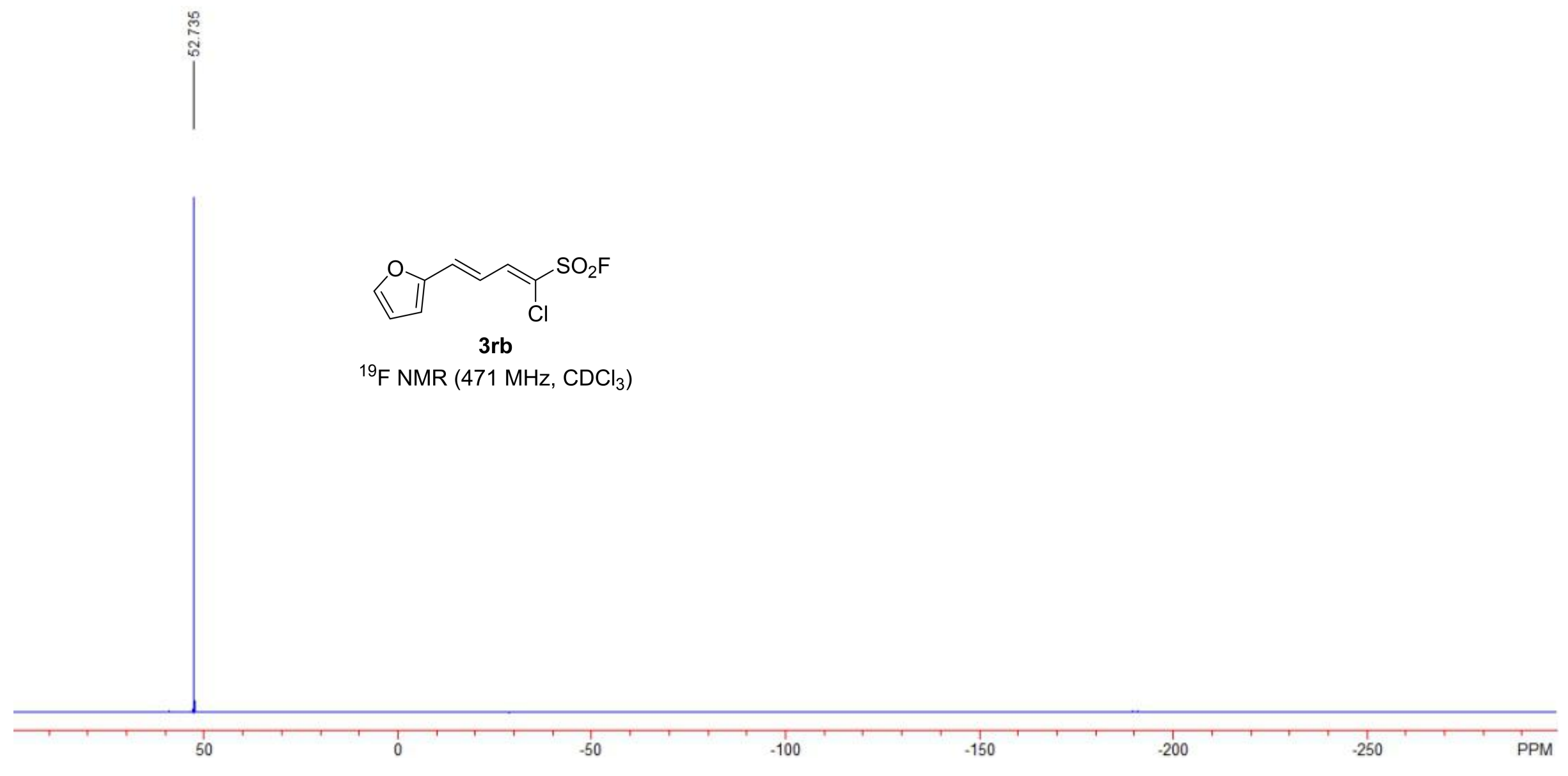




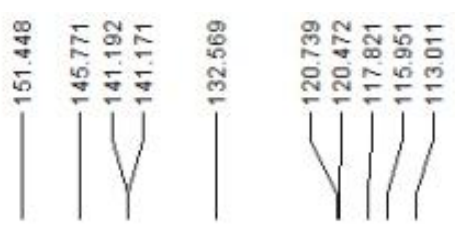

옹요

중

V

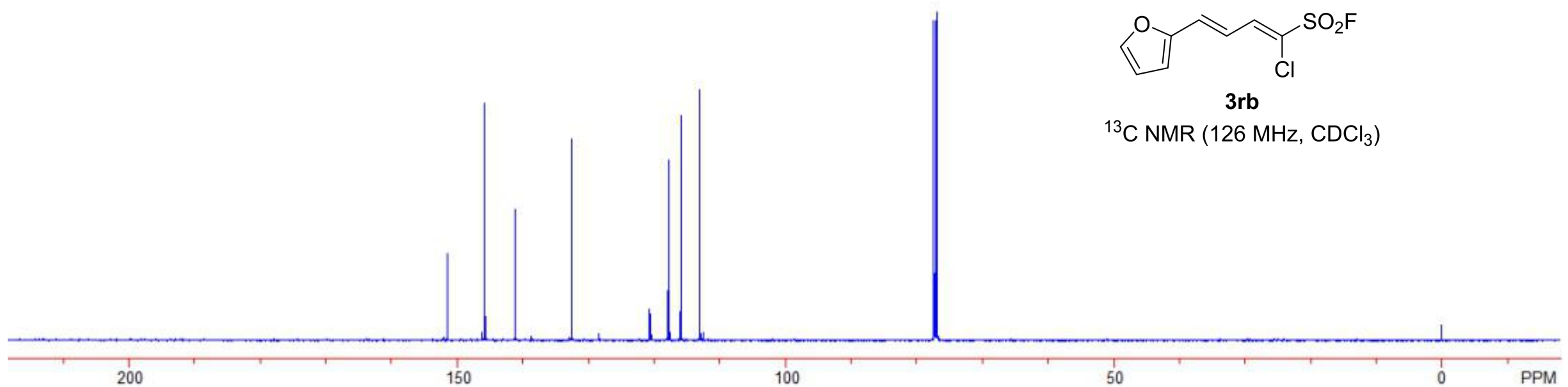




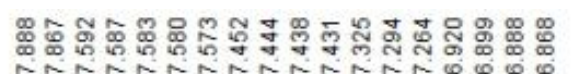

प्रा

D Ilif

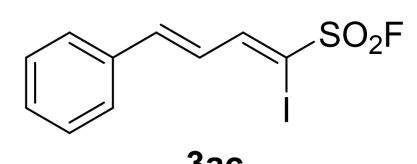

3ac

${ }^{1} \mathrm{H}$ NMR $\left(500 \mathrm{MHz}, \mathrm{CDCl}_{3}\right.$ )

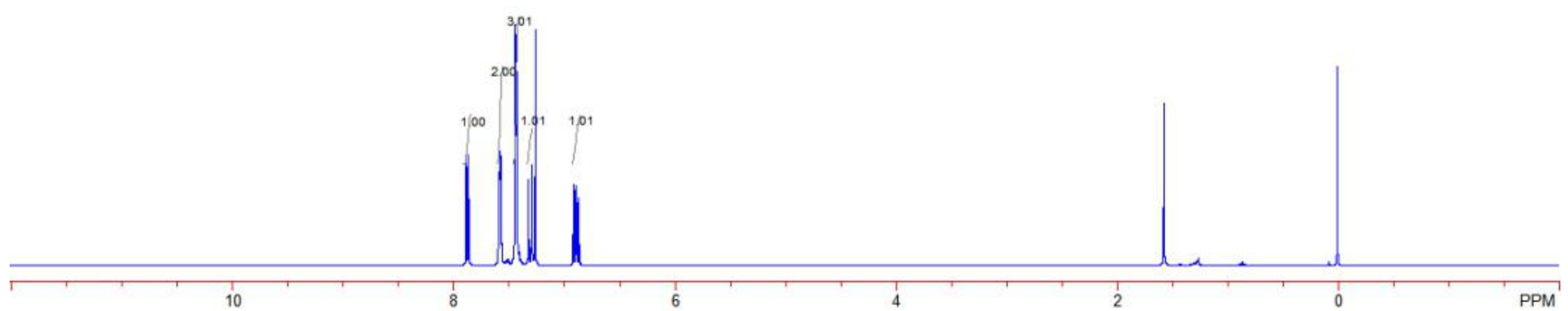



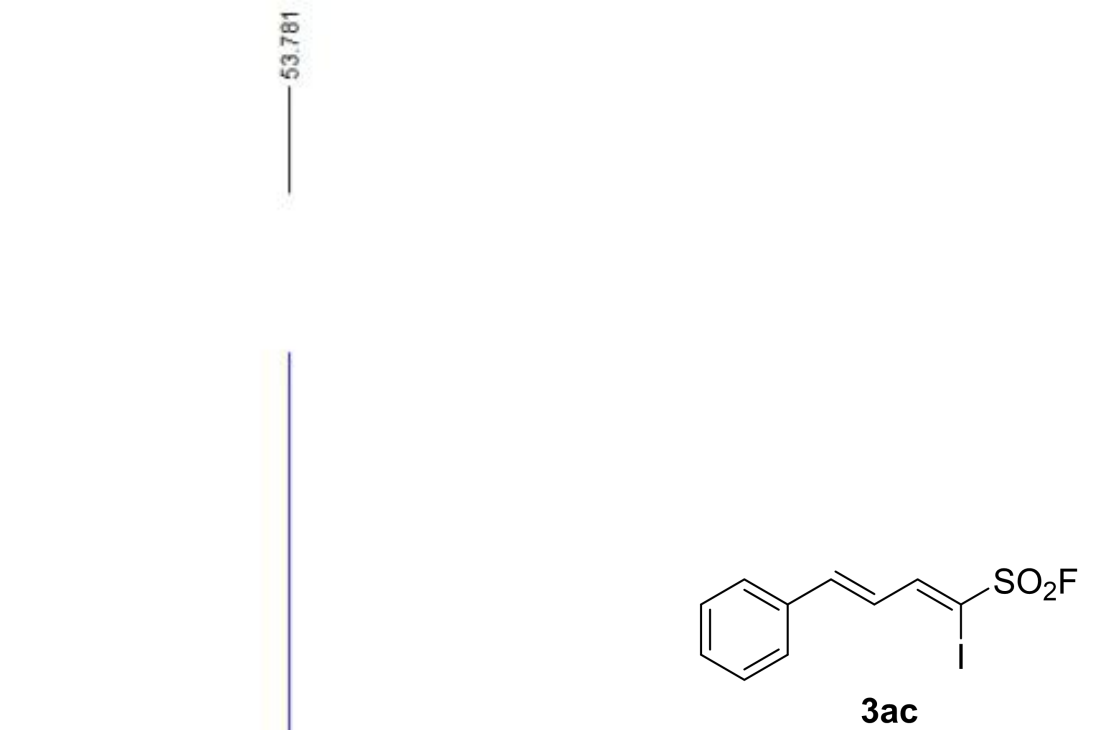

${ }^{19} \mathrm{~F} \mathrm{NMR}\left(471 \mathrm{MHz}, \mathrm{CDCl}_{3}\right)$ 


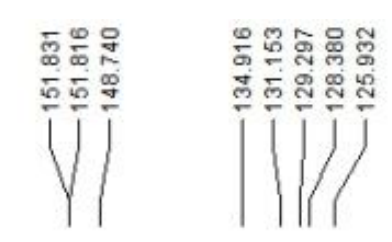

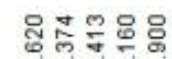

吅

UV

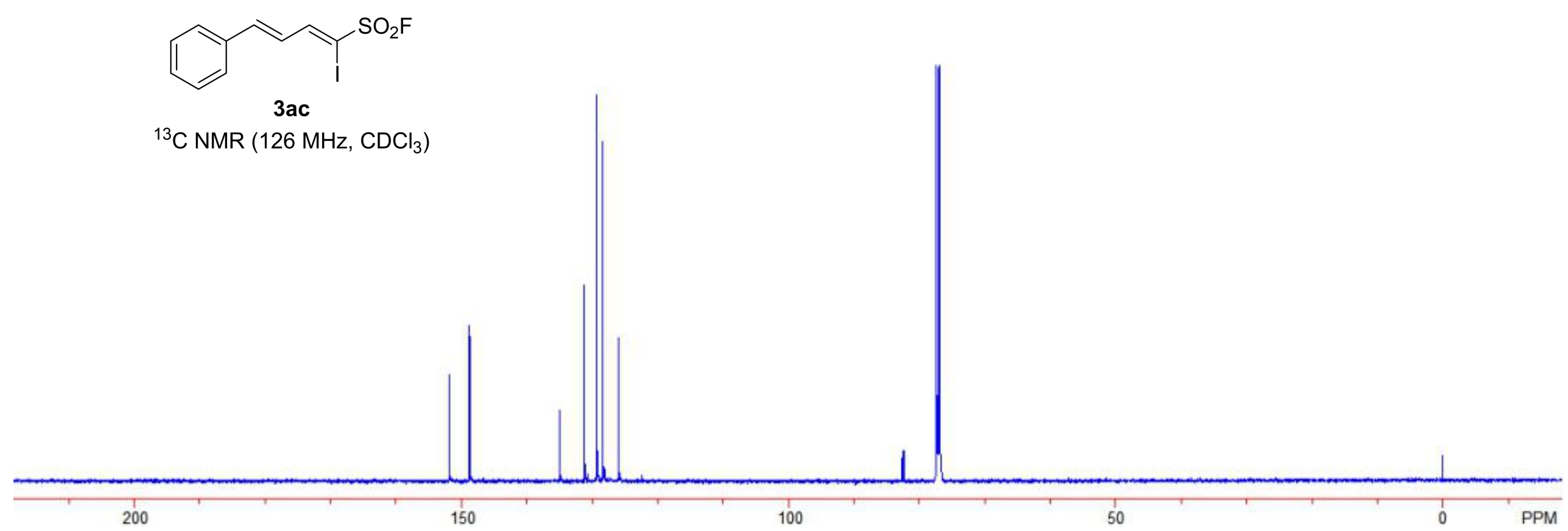



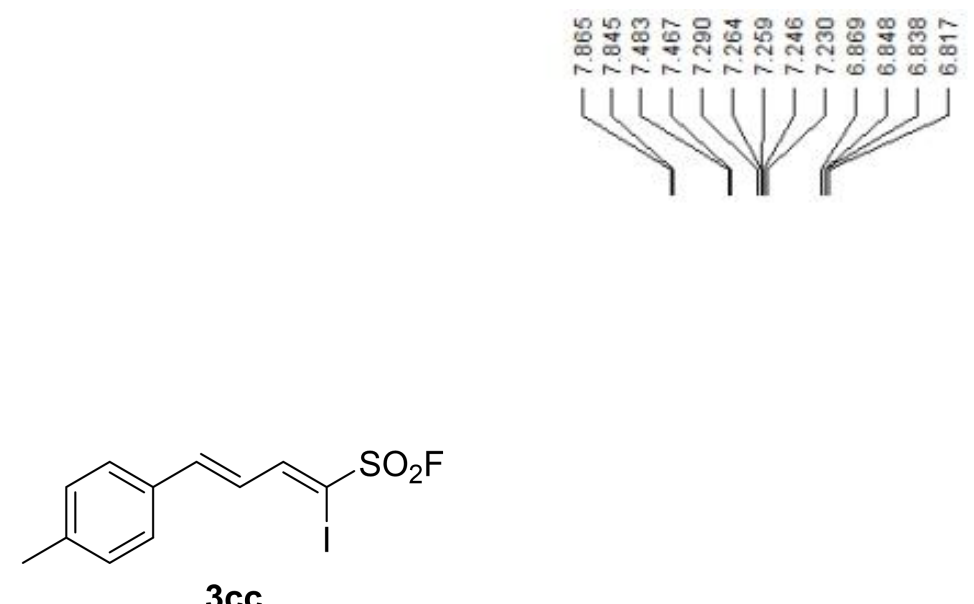

${ }^{1} \mathrm{H}$ NMR $\left(500 \mathrm{MHz}, \mathrm{CDCl}_{3}\right)$

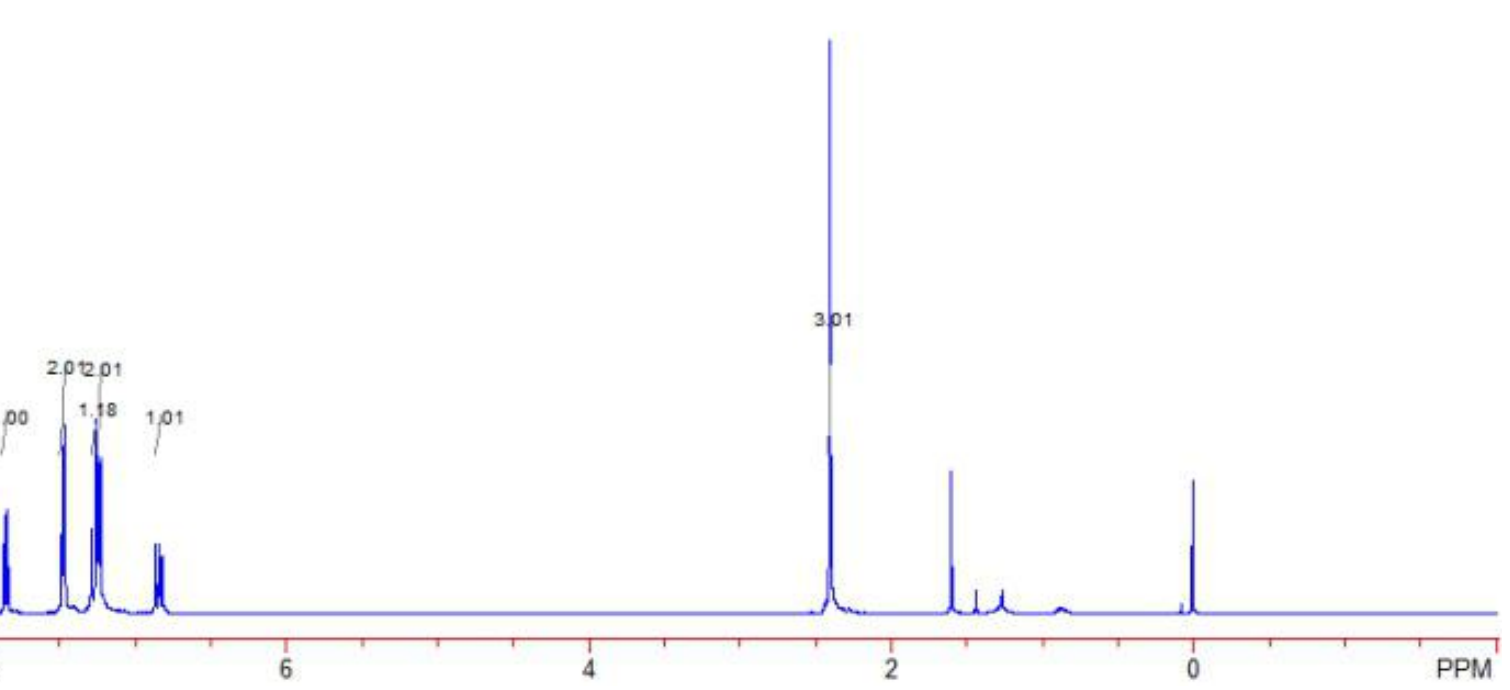




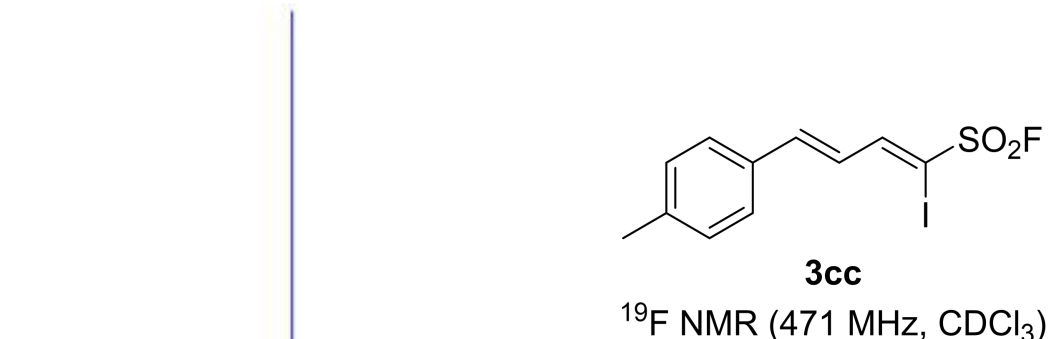

${ }^{19} \mathrm{~F} \mathrm{NMR}\left(471 \mathrm{MHz}, \mathrm{CDCl}_{3}\right)$ 


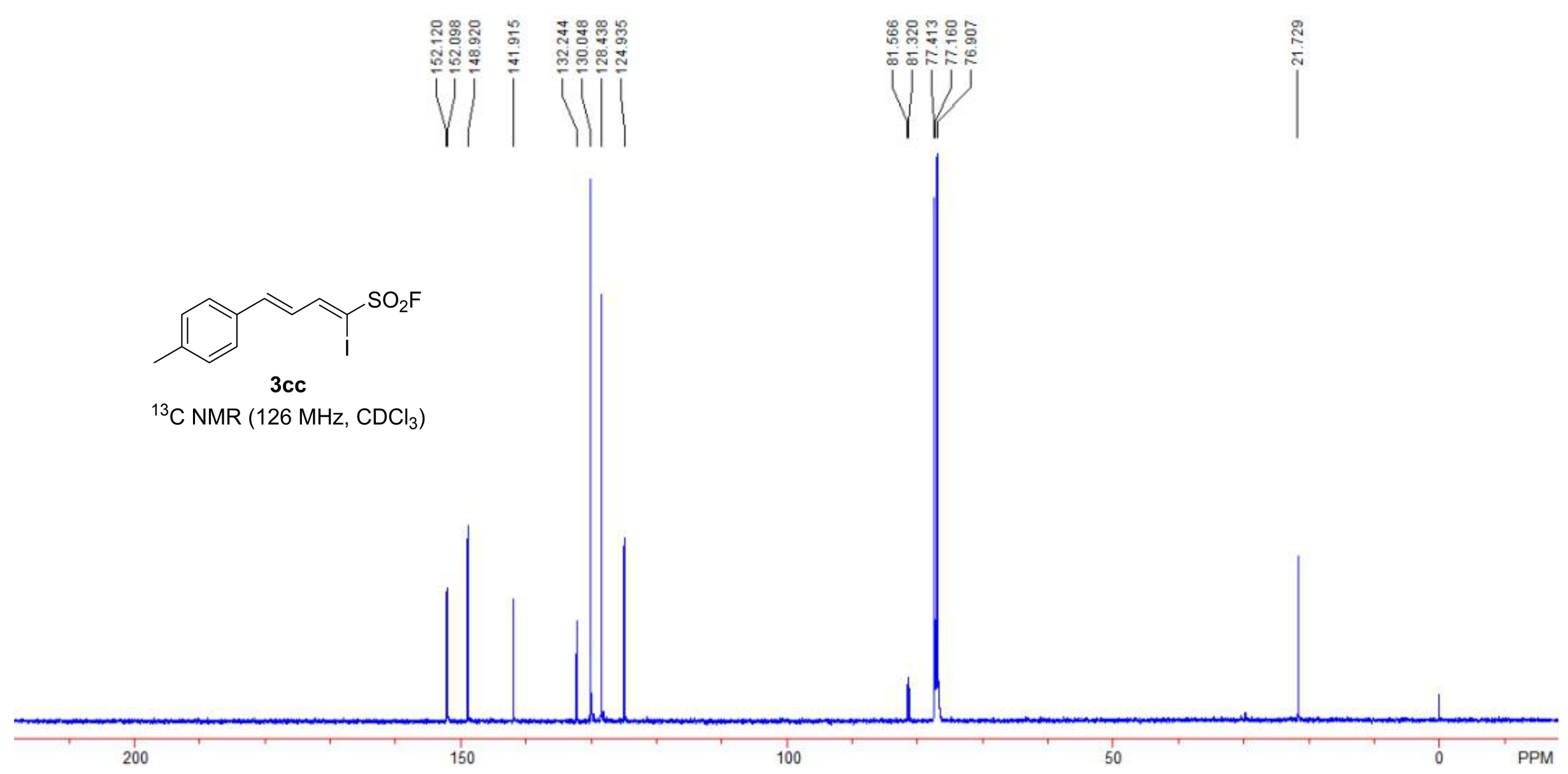




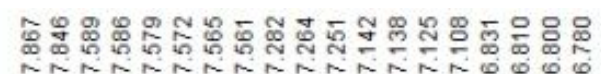 \\ LMUUUNय \\ $\rightarrow$ II}

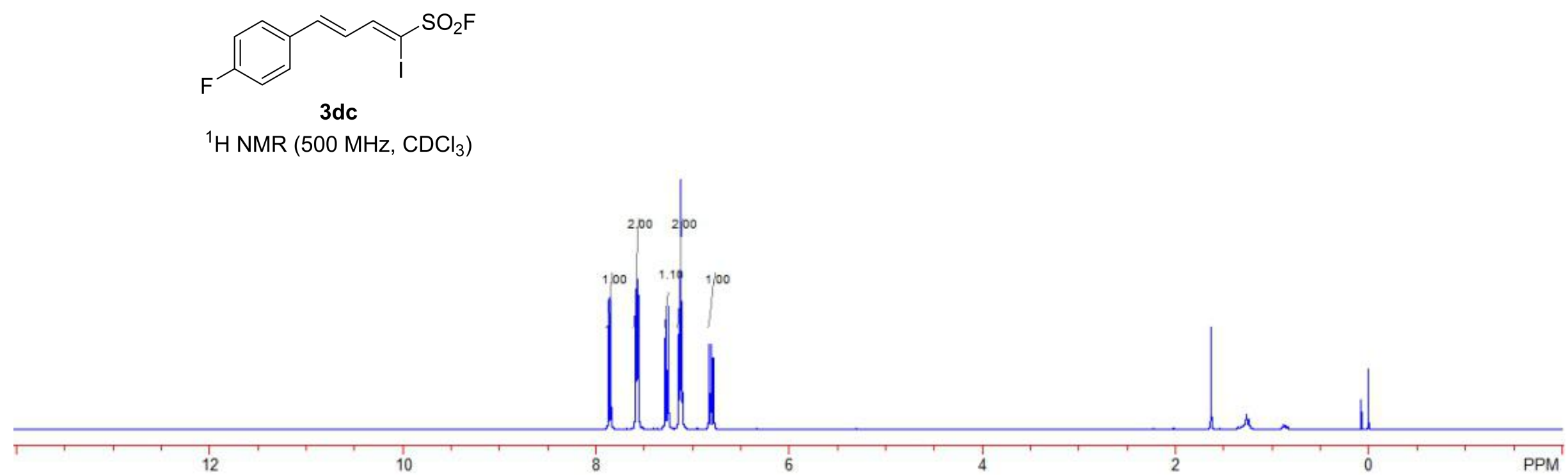




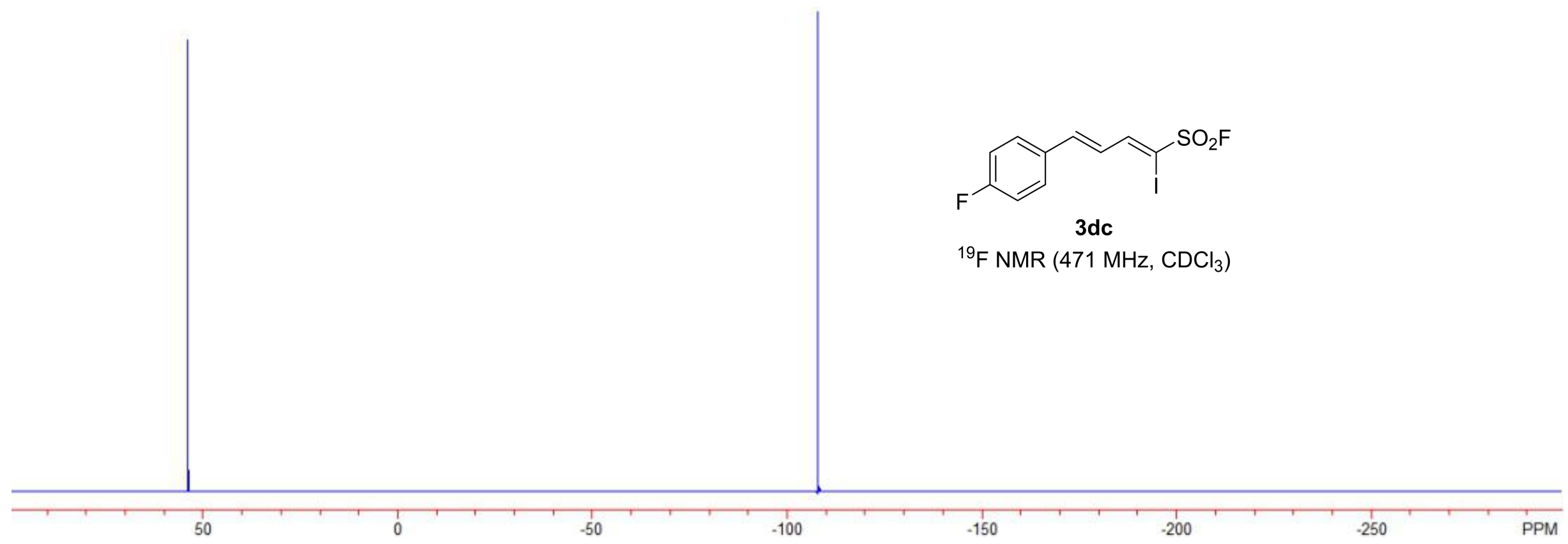




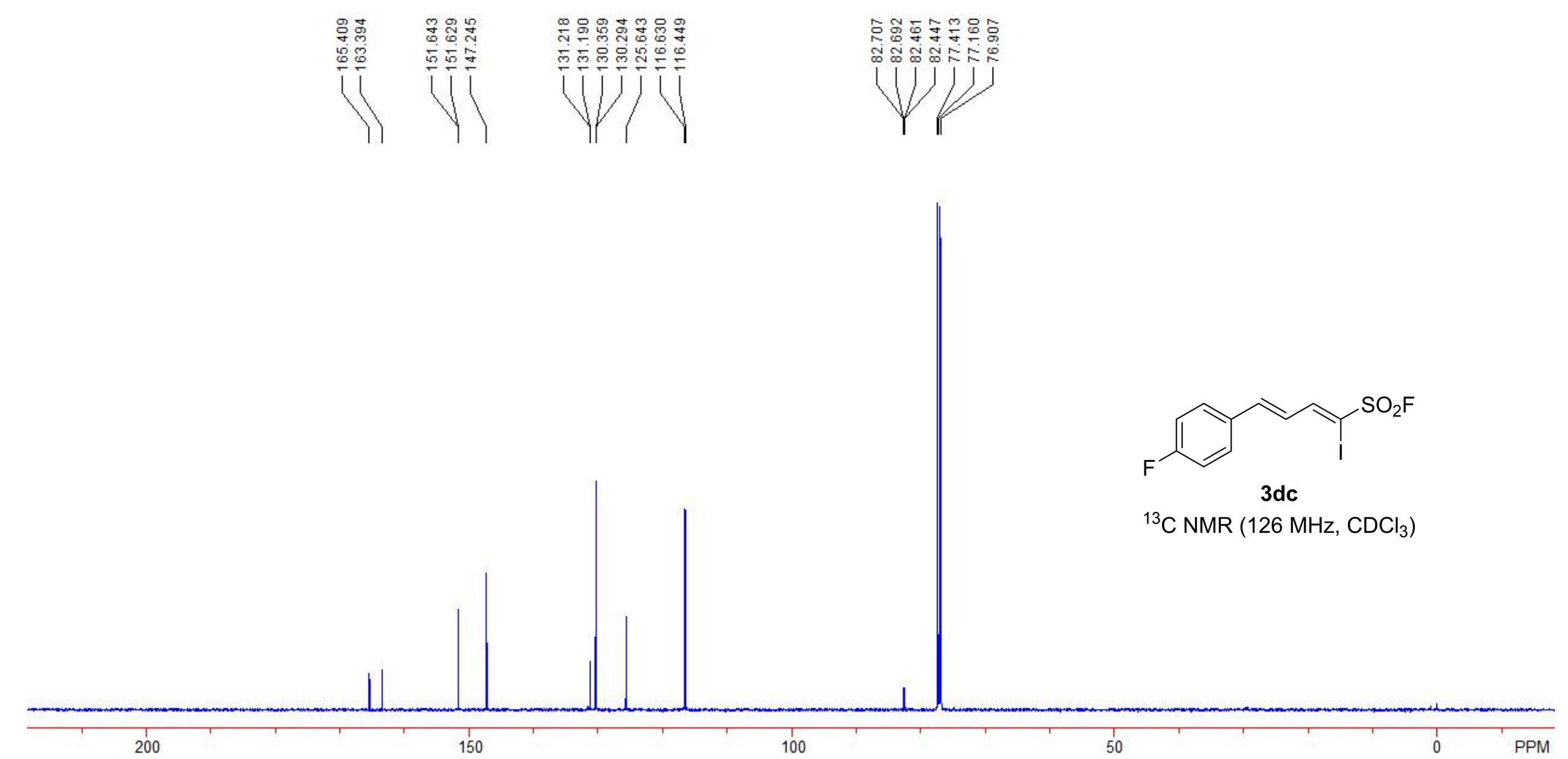




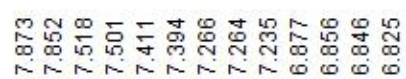

यU1111011

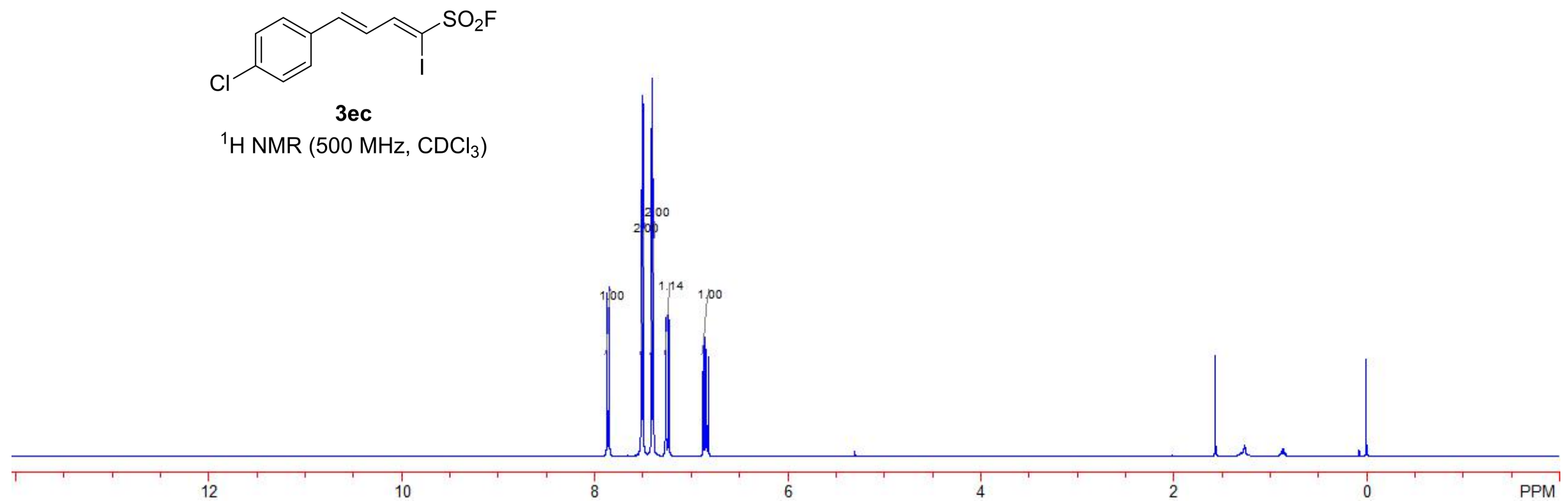




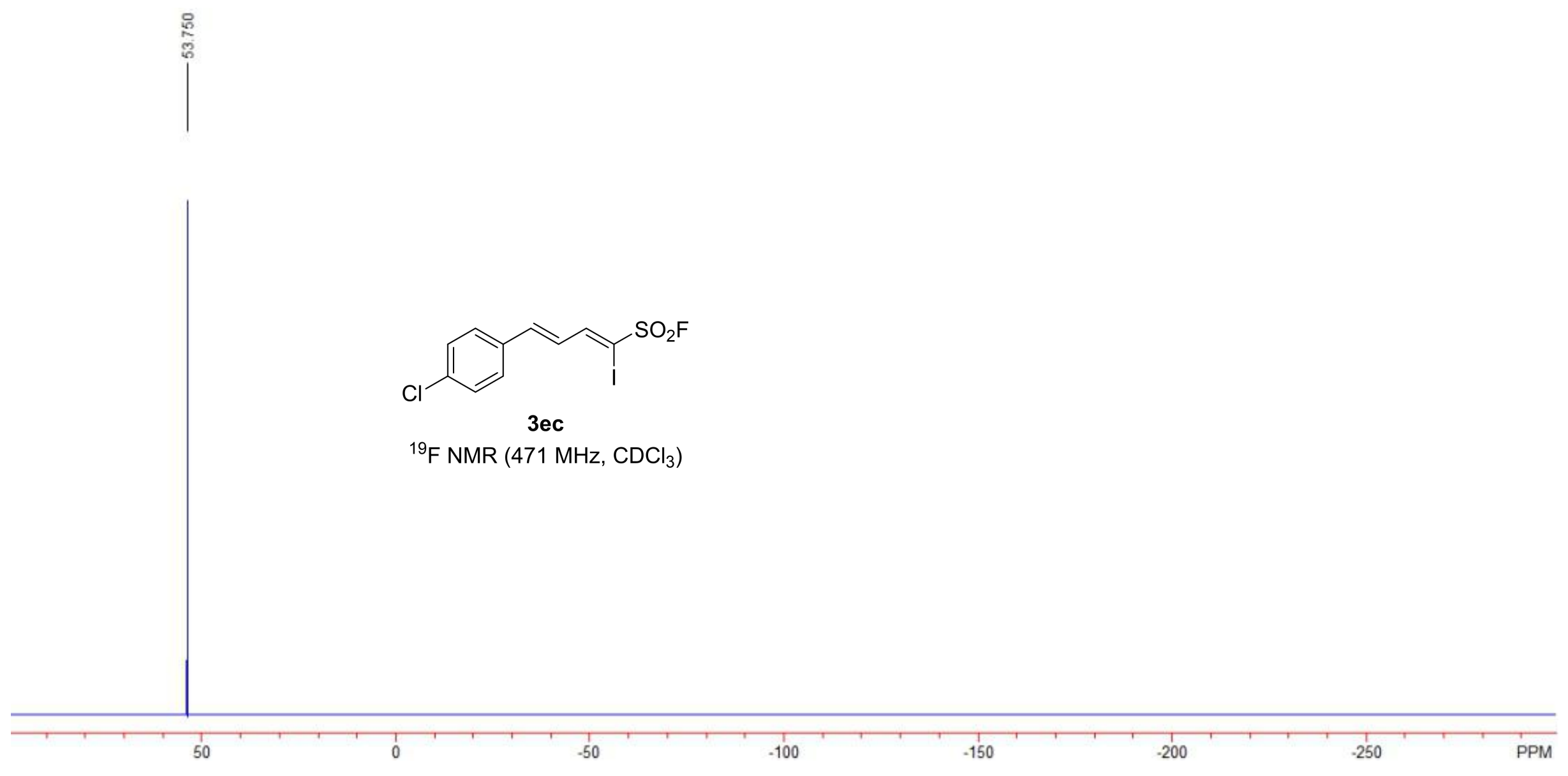



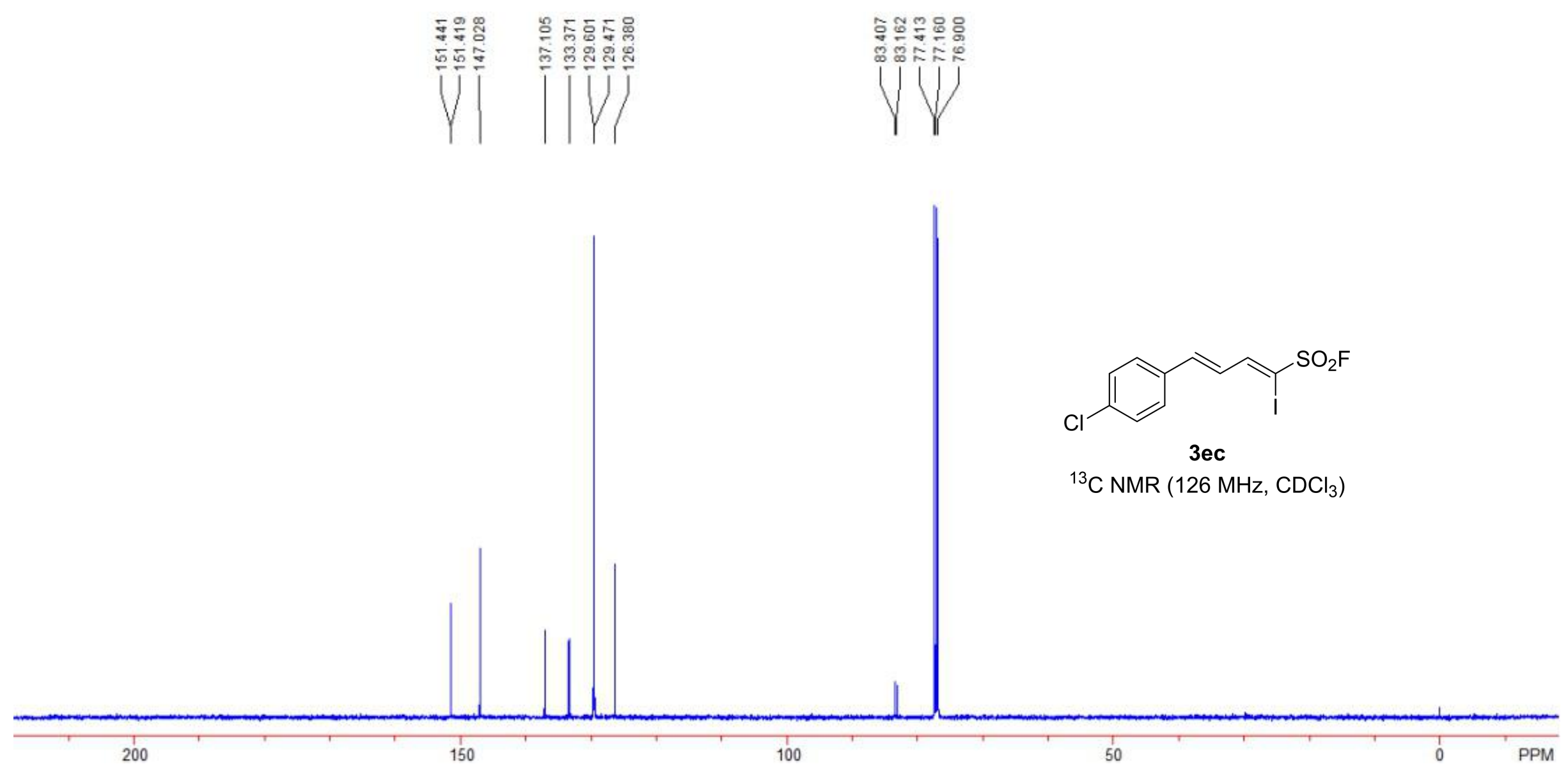


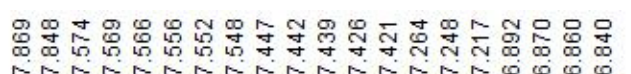

$L L L L L L(1) J J\rfloor J\rfloor J\rfloor$

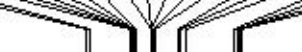

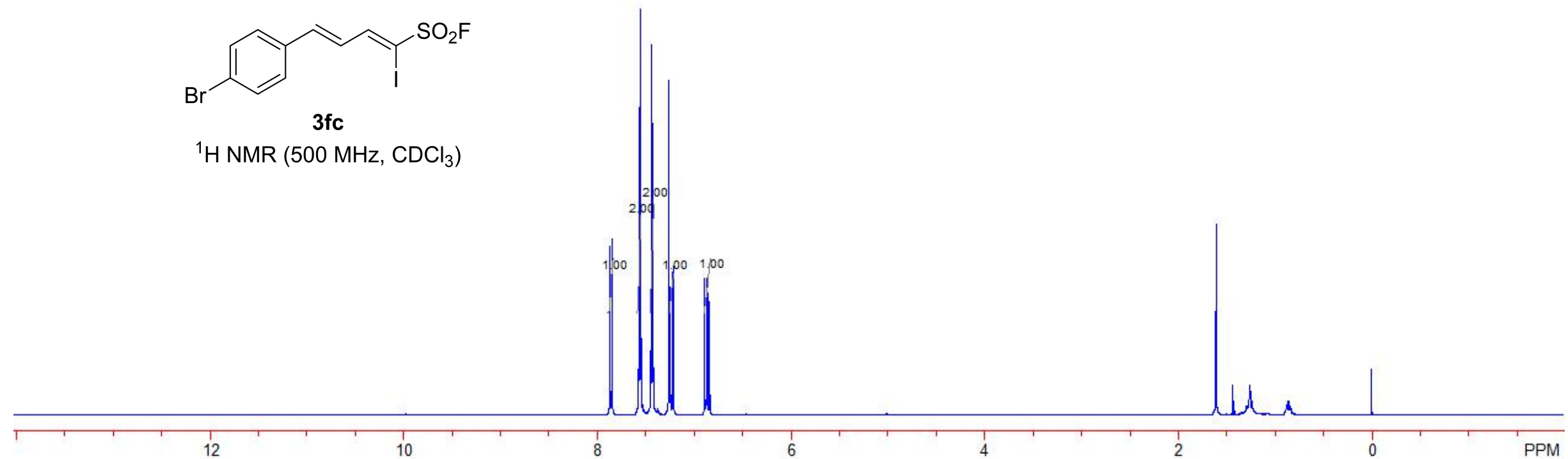




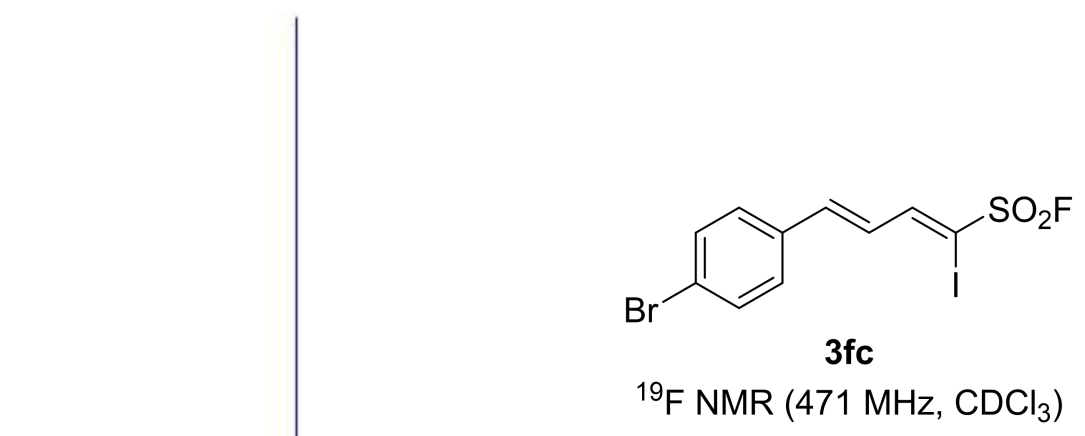




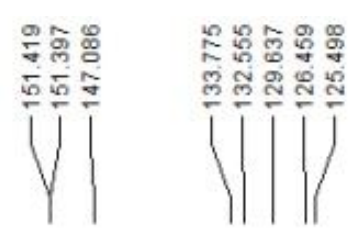

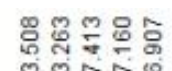

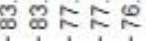

V

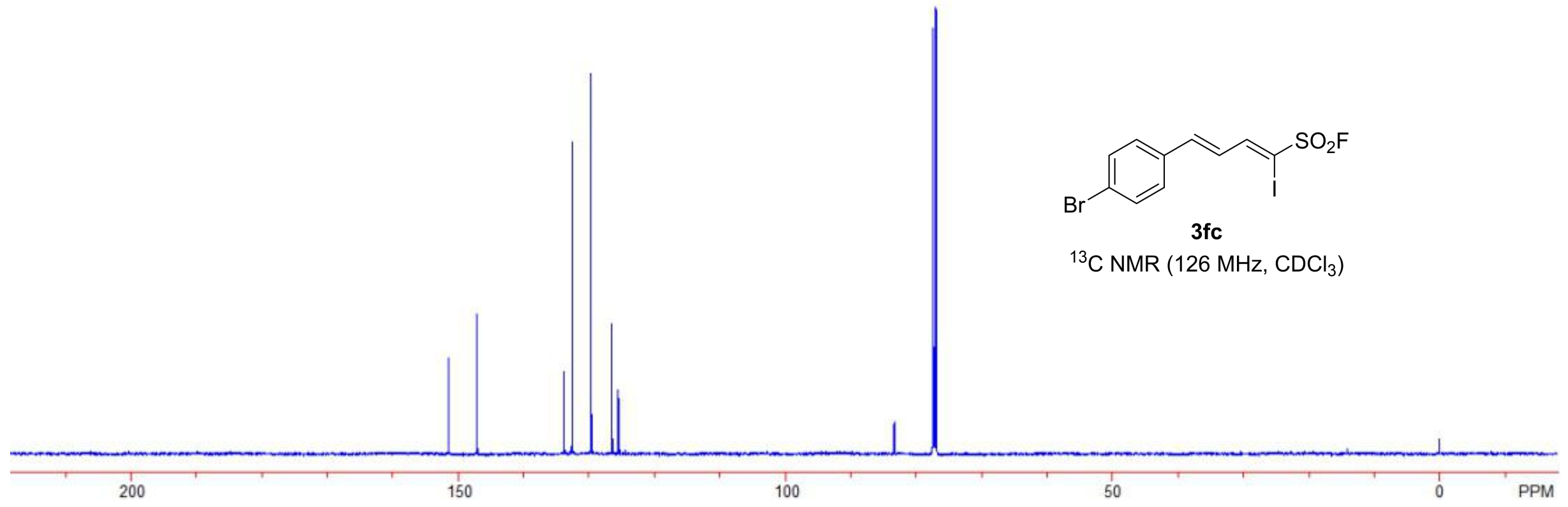




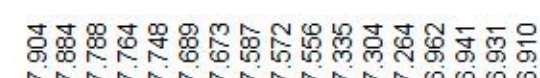

ULUUUJ J J J

nitil II

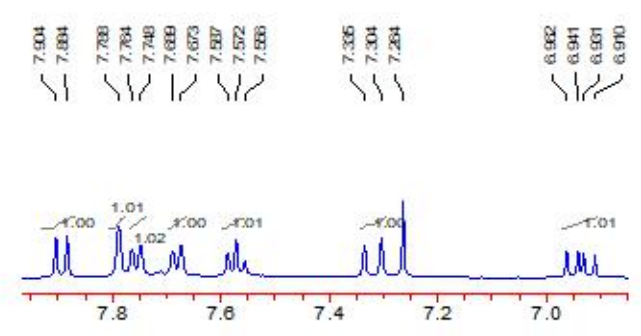

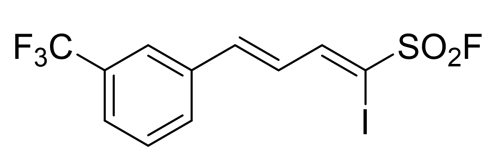

$3 \mathrm{kc}$

${ }^{1} \mathrm{H} \mathrm{NMR}\left(500 \mathrm{MHz}, \mathrm{CDCl}_{3}\right)$

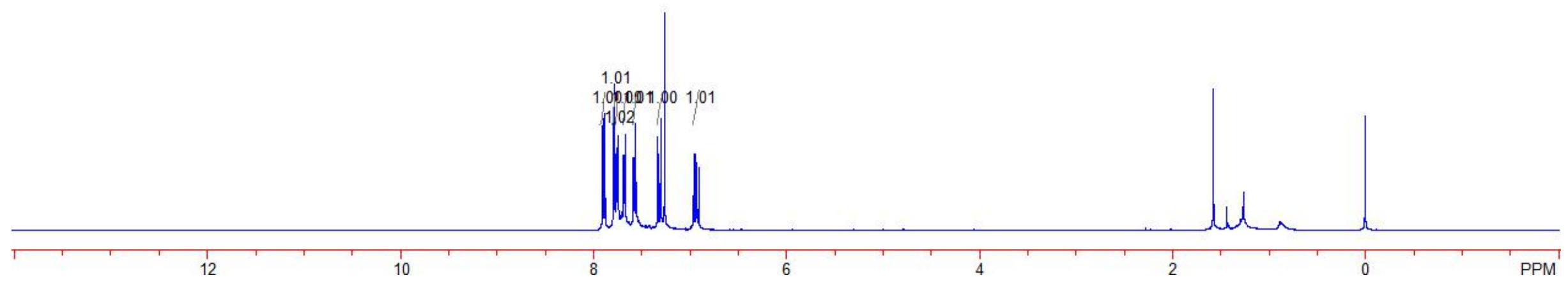




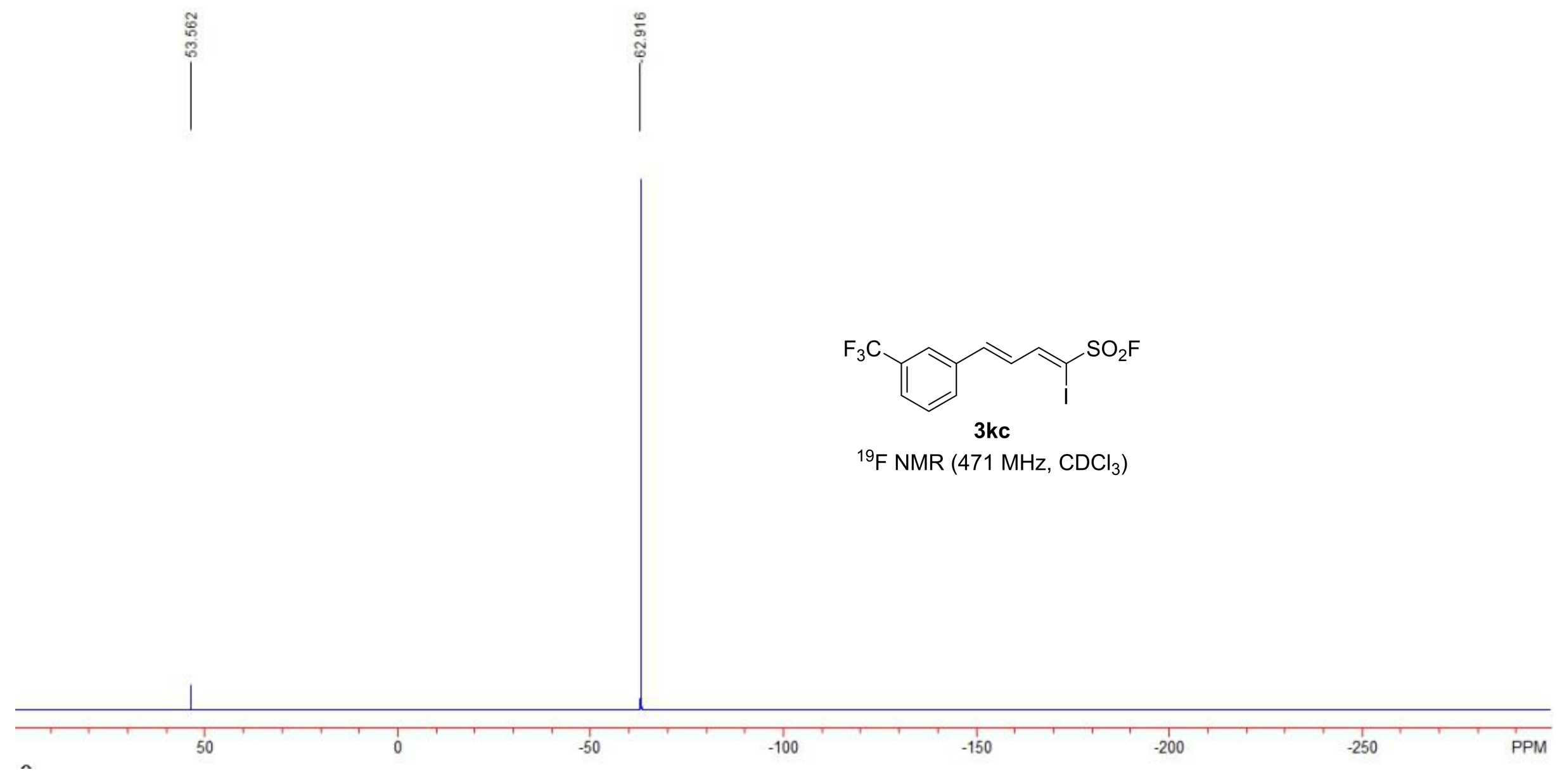




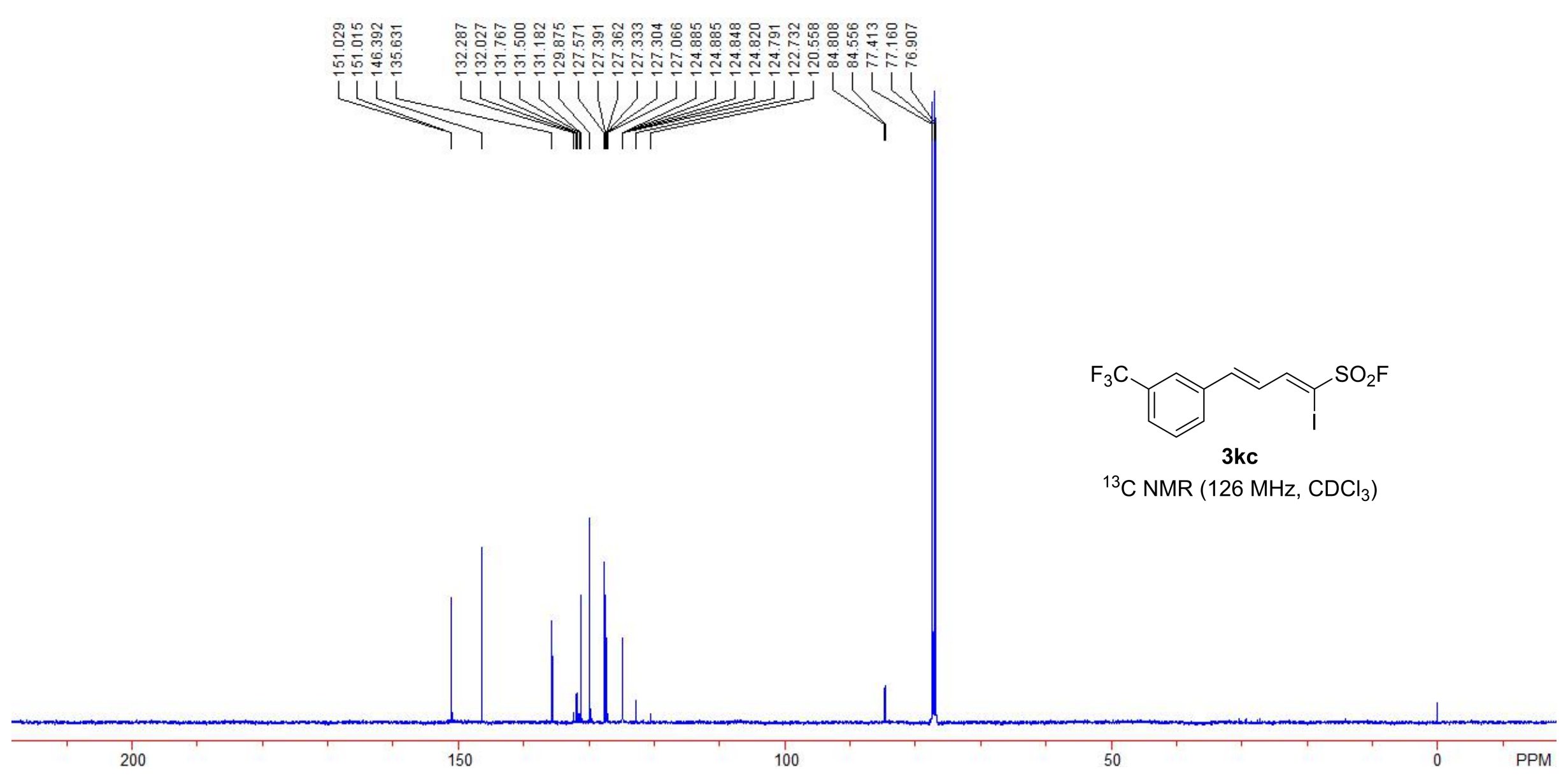




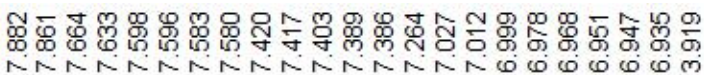

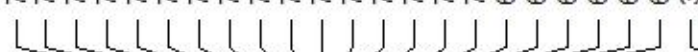
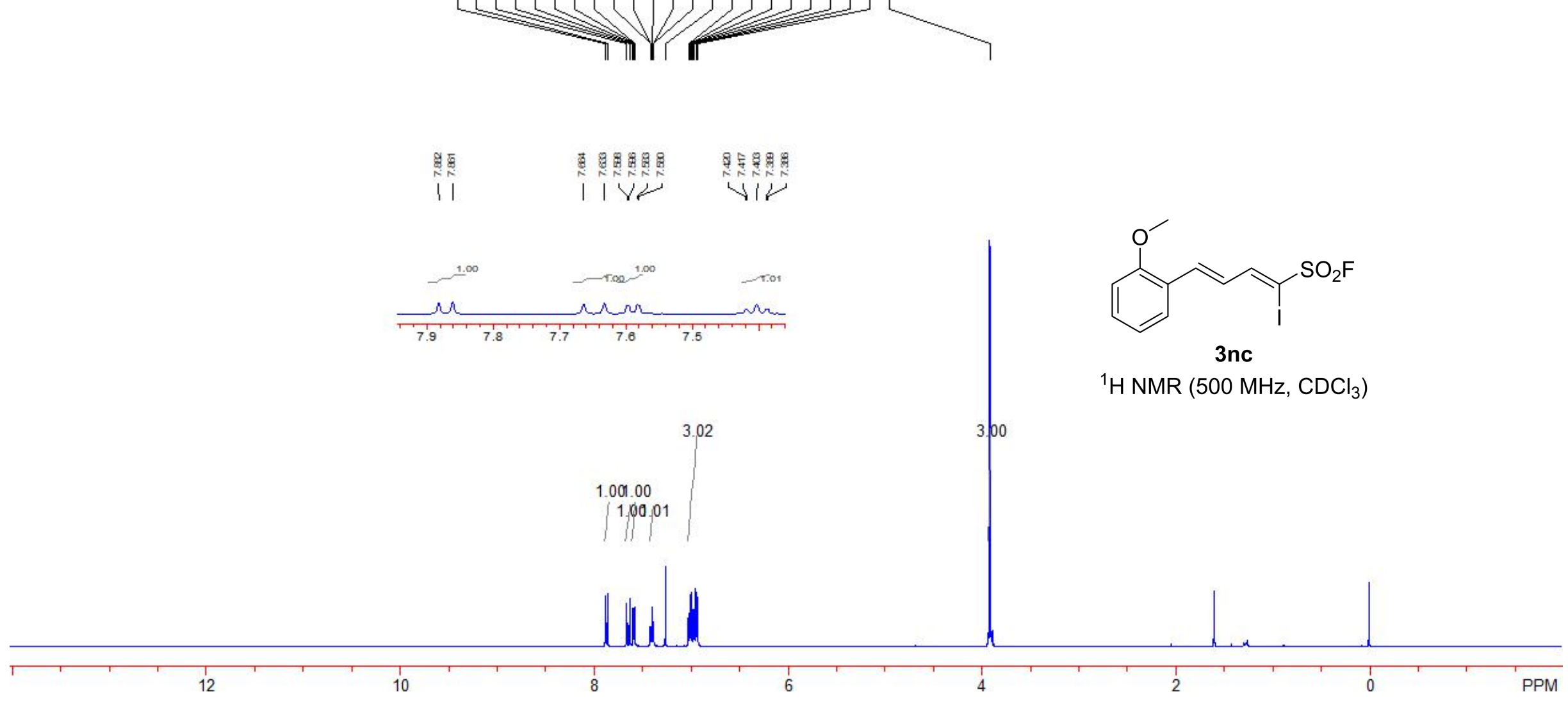


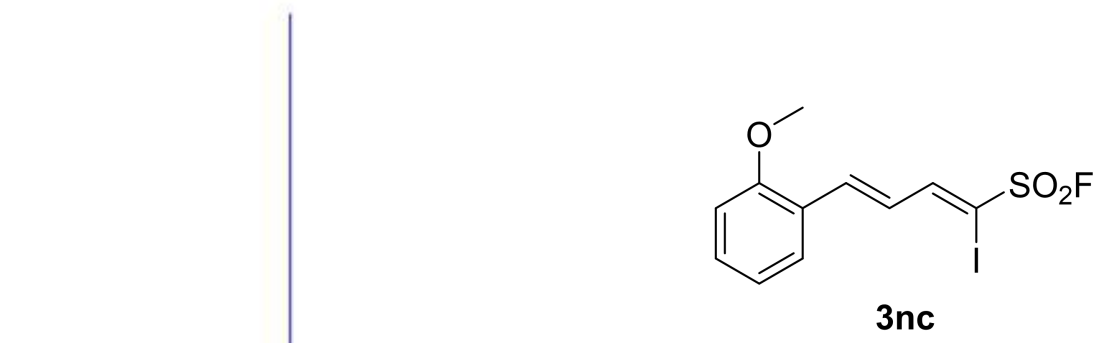

${ }^{19} \mathrm{~F}$ NMR $\left(471 \mathrm{MHz}, \mathrm{CDCl}_{3}\right)$ 

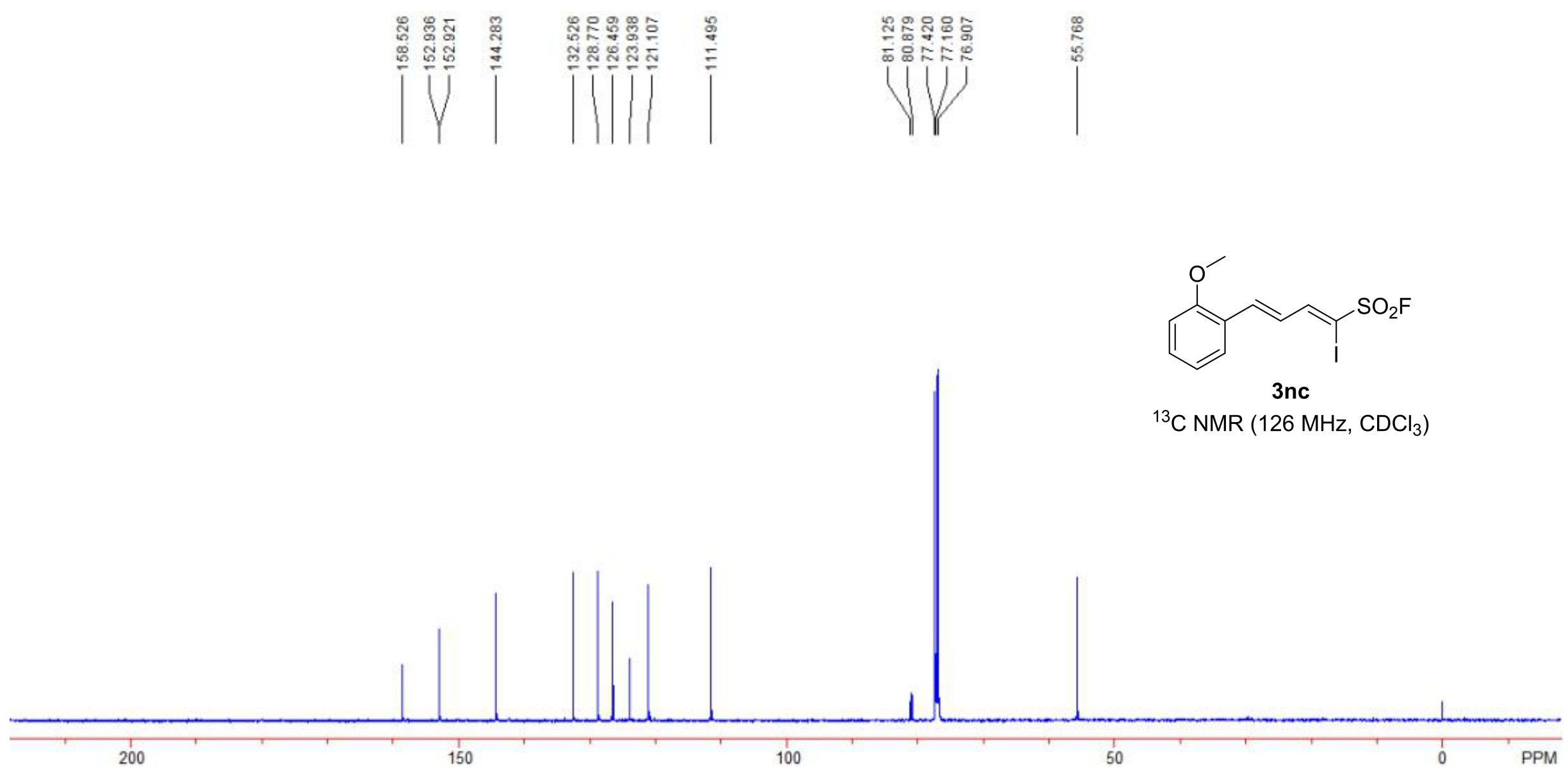

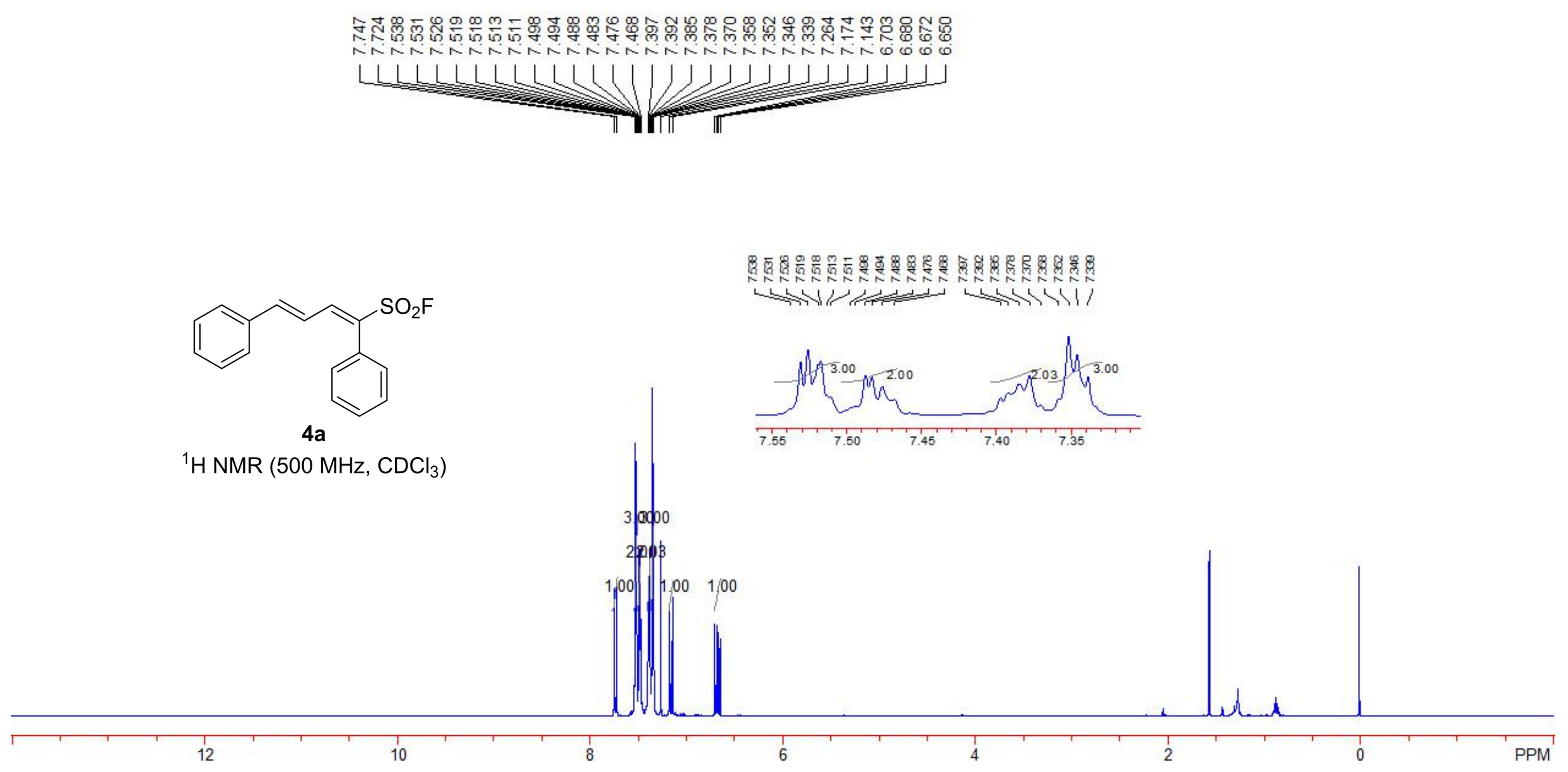

S129 


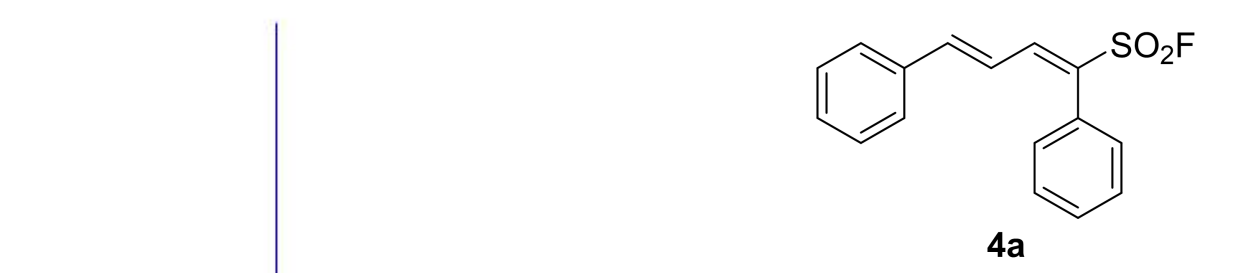

${ }^{19} \mathrm{~F}$ NMR $\left(471 \mathrm{MHz}, \mathrm{CDCl}_{3}\right)$ 


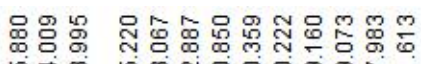

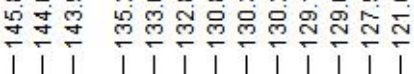

M N

举吕管

$\therefore \pi$

U

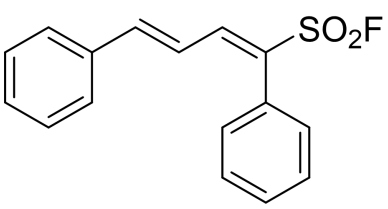

4a

${ }^{13} \mathrm{C}$ NMR $\left(126 \mathrm{MHz}, \mathrm{CDCl}_{3}\right)$ 

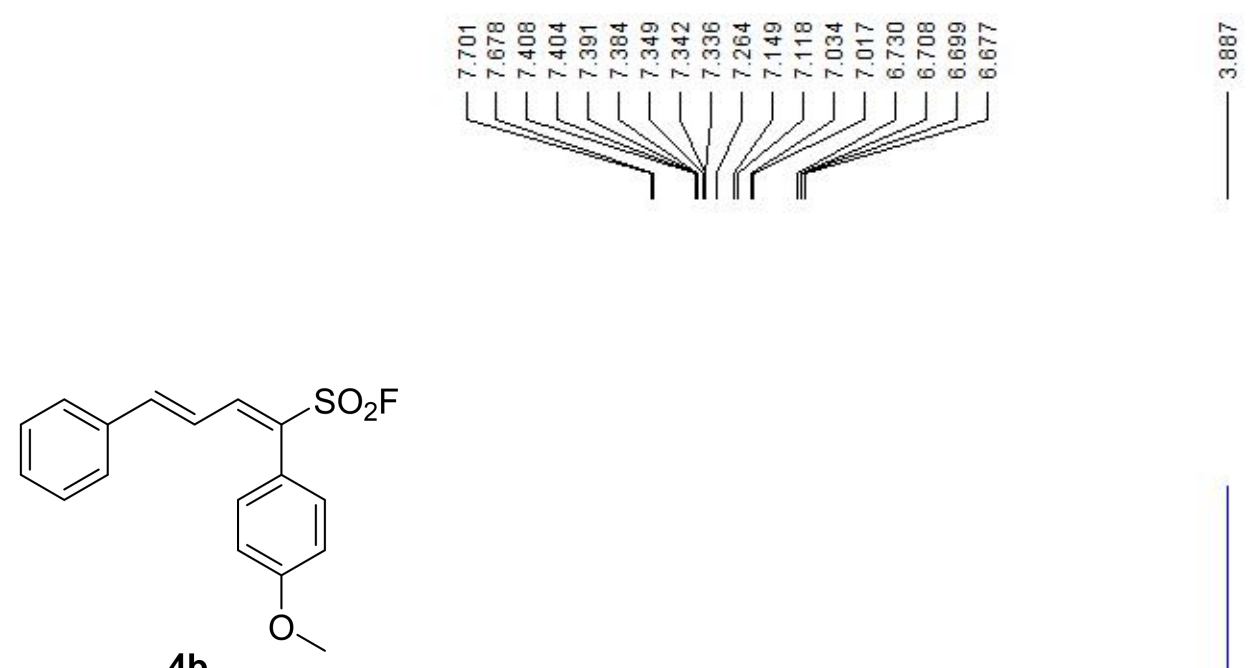

${ }^{1} \mathrm{H}$ NMR $\left(500 \mathrm{MHz}, \mathrm{CDCl}_{3}\right)$

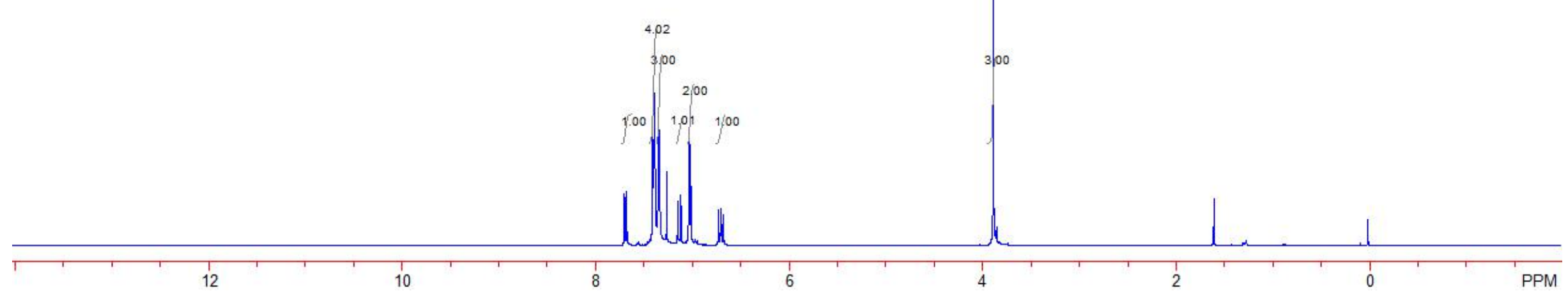



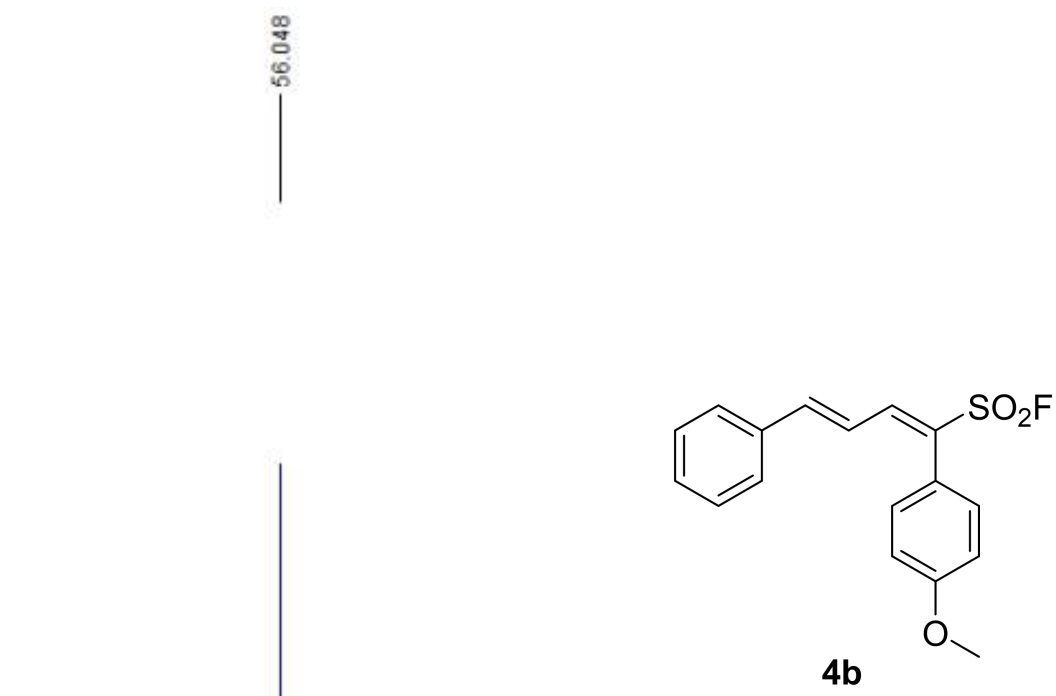

${ }^{19} \mathrm{~F}$ NMR $\left(471 \mathrm{MHz}, \mathrm{CDCl}_{3}\right)$

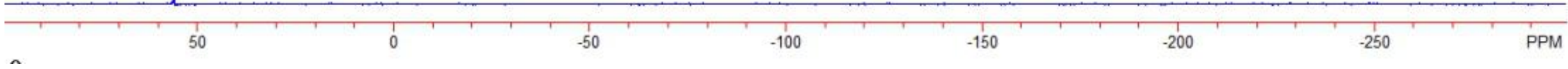



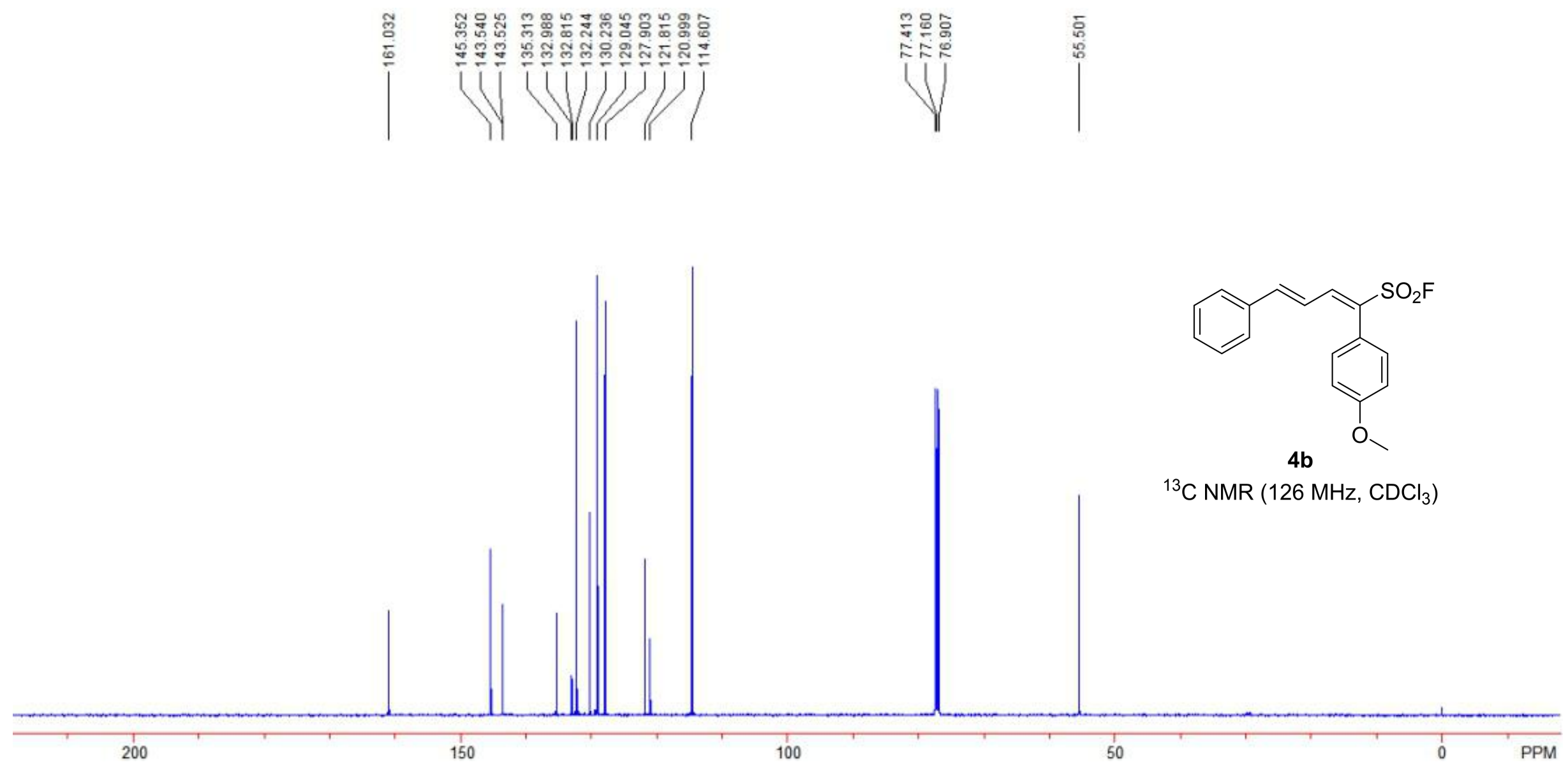

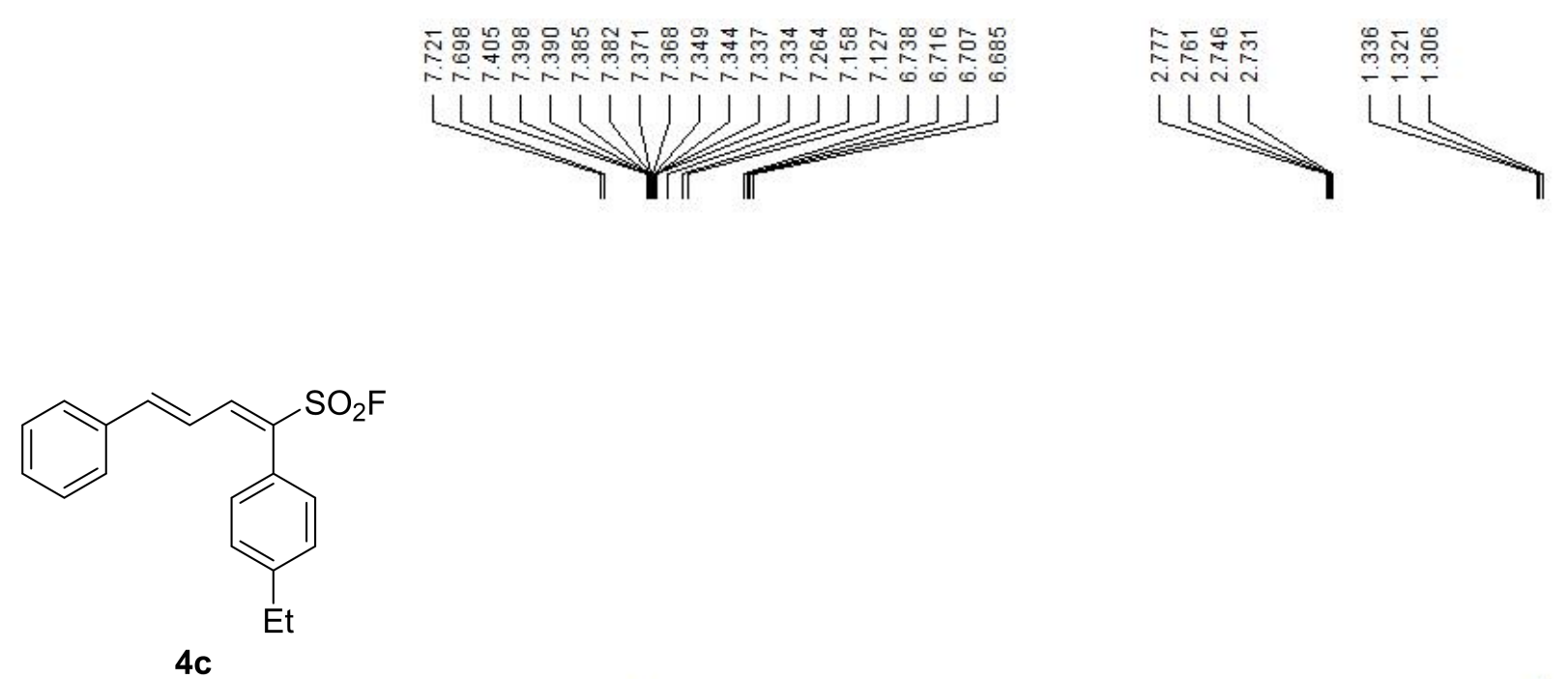

${ }^{1} \mathrm{H}$ NMR $\left(500 \mathrm{MHz}, \mathrm{CDCl}_{3}\right)$

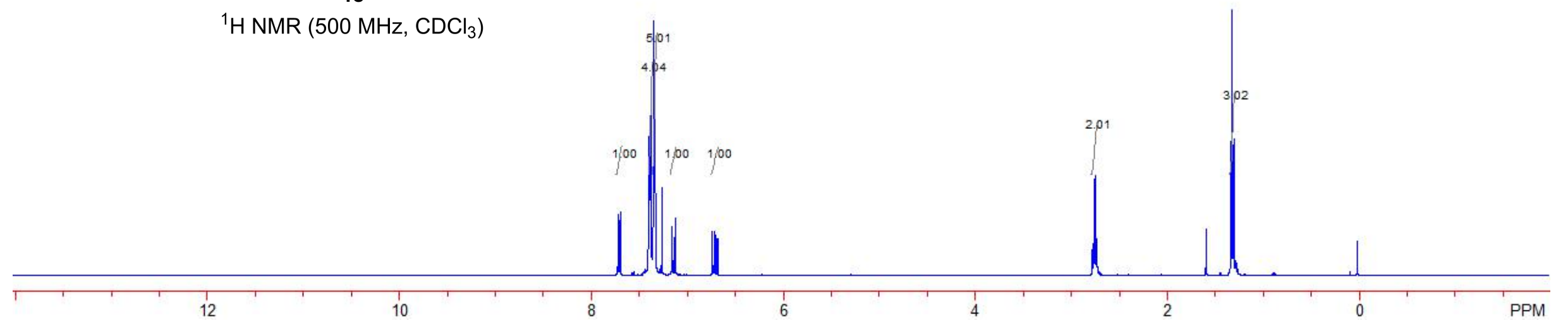




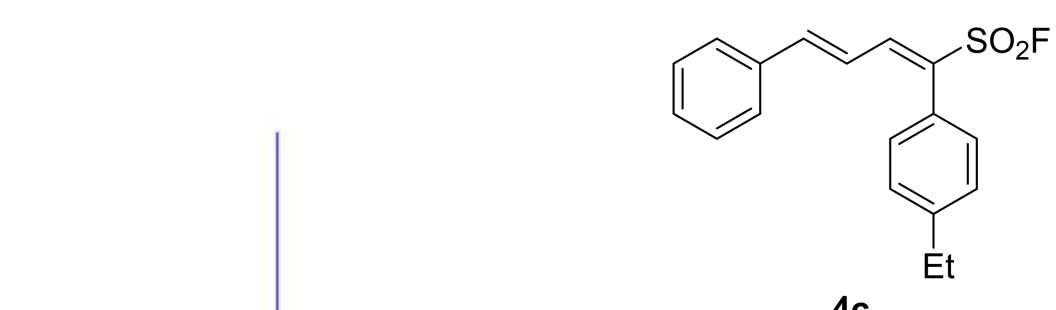

${ }^{19} \mathrm{~F}$ NMR $\left(471 \mathrm{MHz}, \mathrm{CDCl}_{3}\right)$

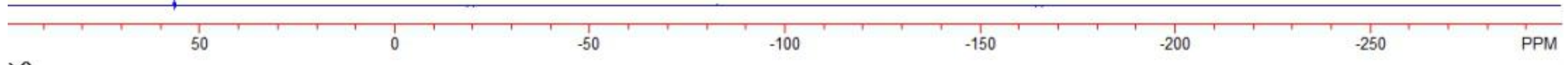




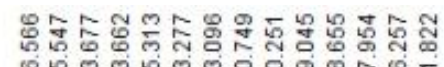

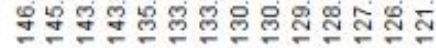
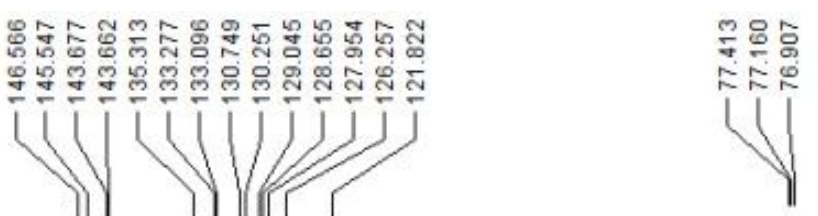

$\left.\right|^{\substack{0 \\ \infty}}$

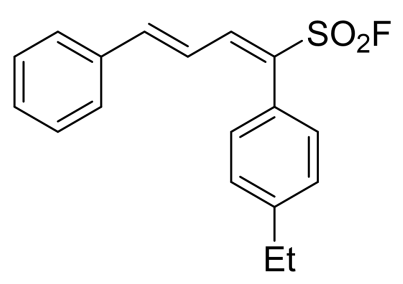

4c

${ }^{13} \mathrm{C}$ NMR $\left(126 \mathrm{MHz}, \mathrm{CDCl}_{3}\right)$ 


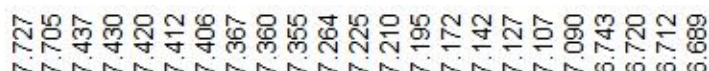 \\ LLLLLUUU|JJJJJJ」J \\ ] $7 \pi_{\pi}$}
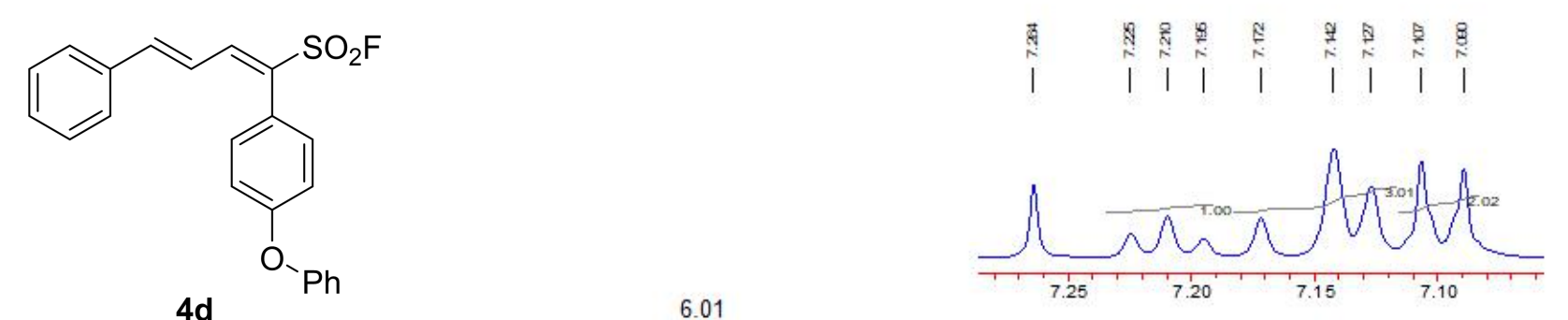

${ }^{1} \mathrm{H}$ NMR $\left(500 \mathrm{MHz}, \mathrm{CDCl}_{3}\right)$

6.01

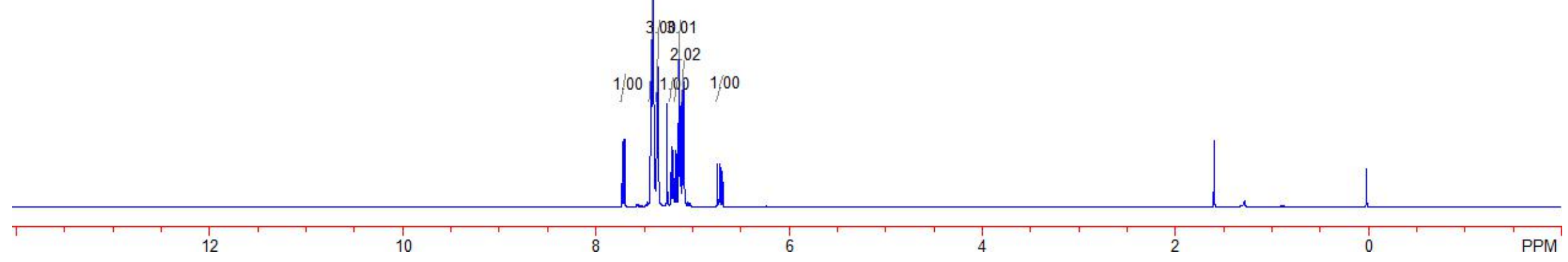




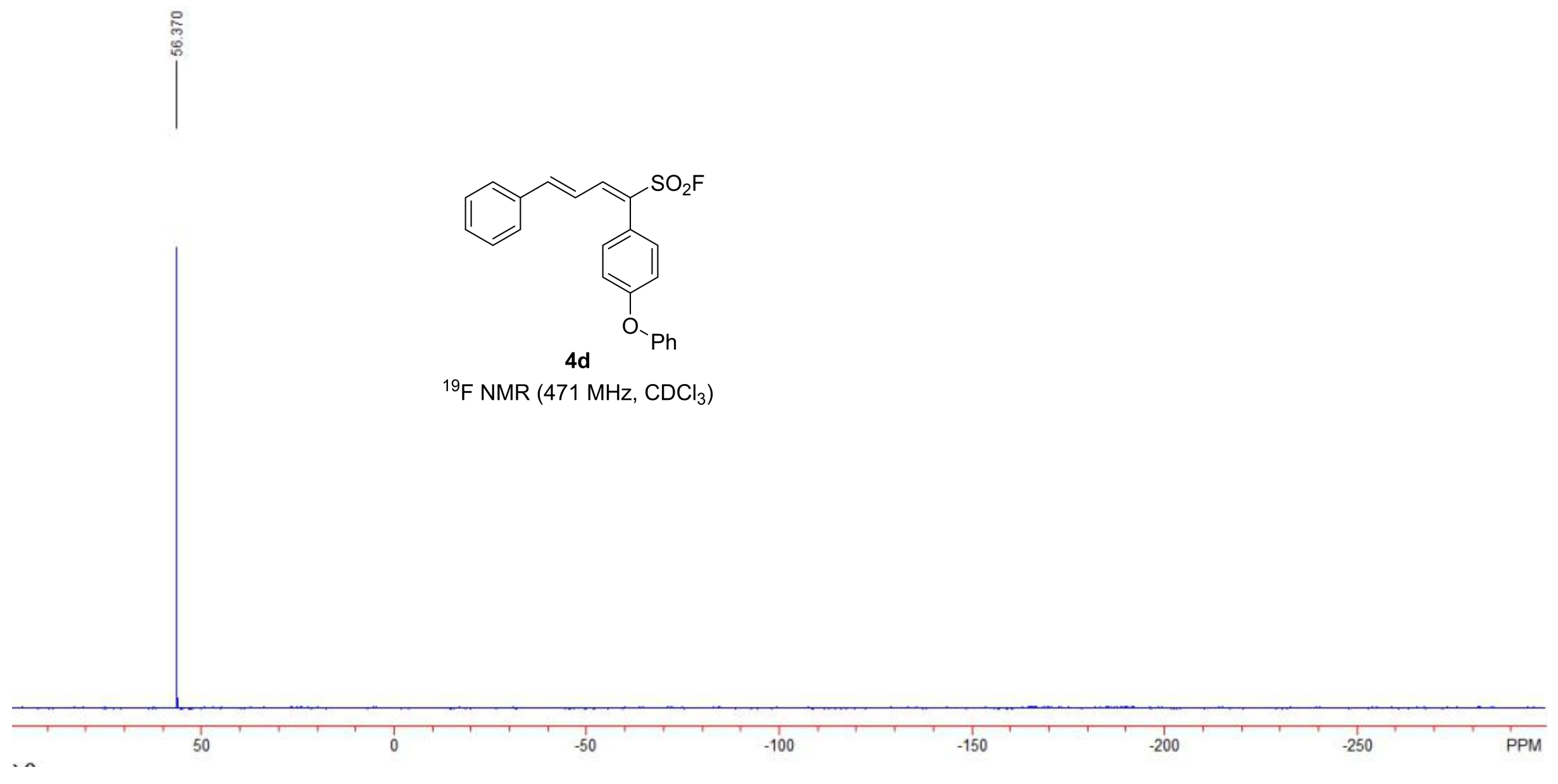



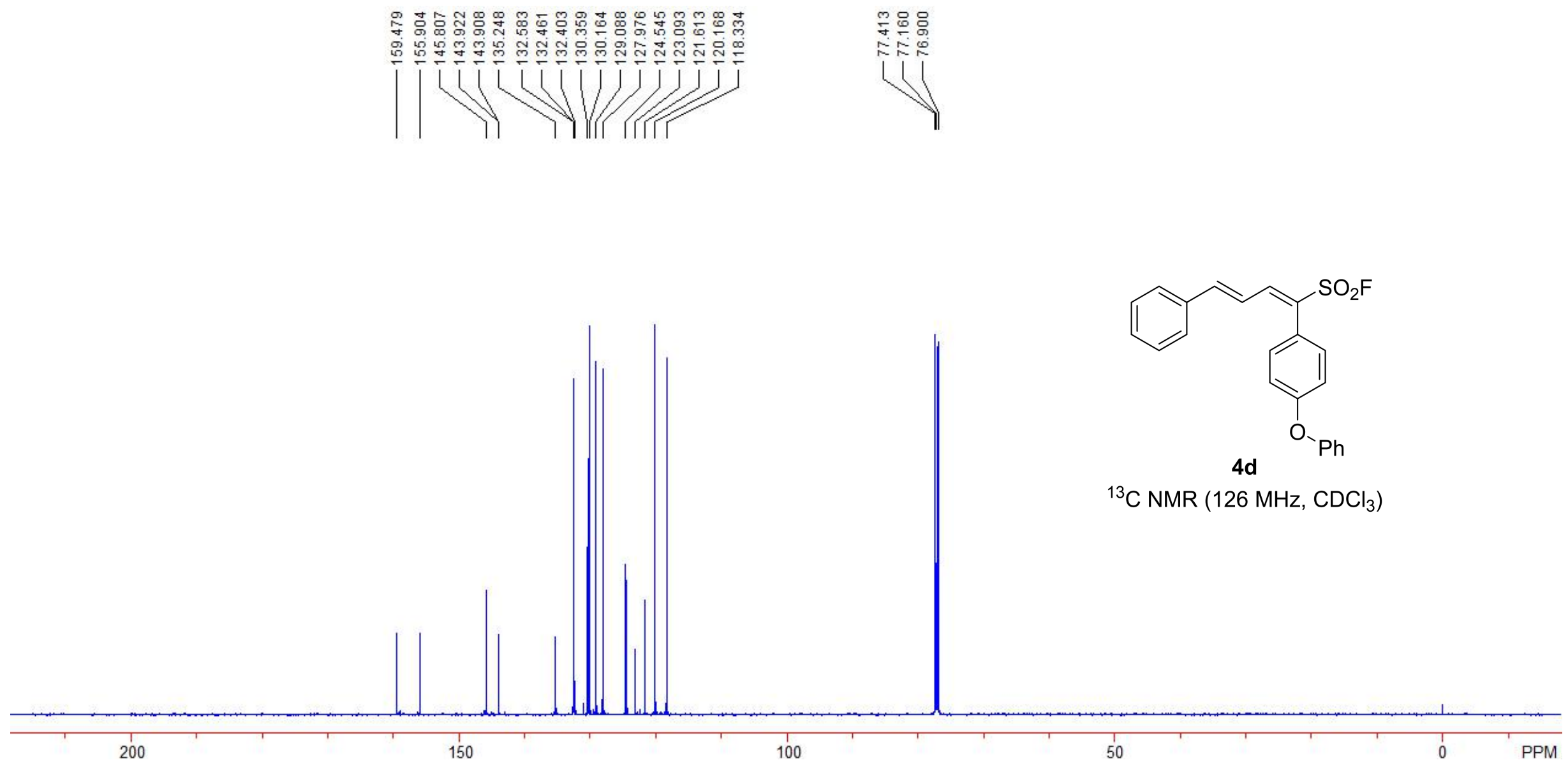


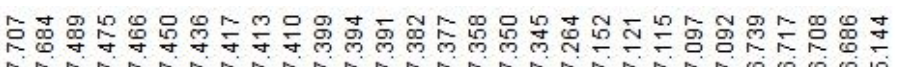

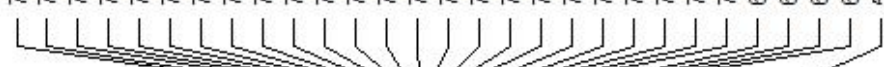

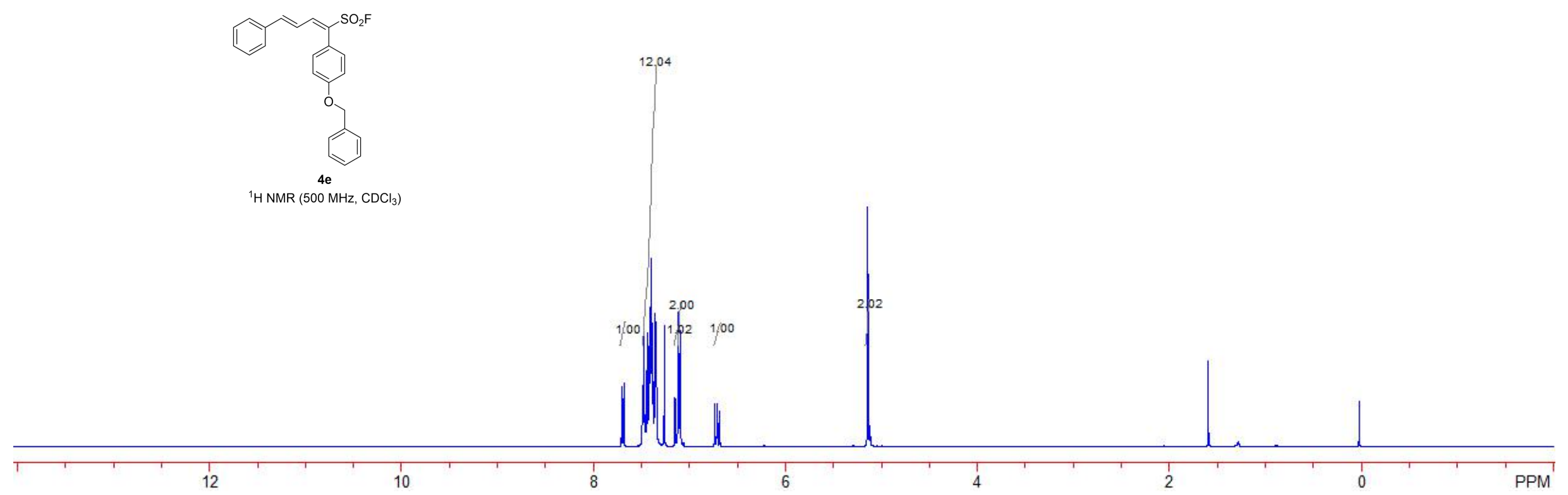




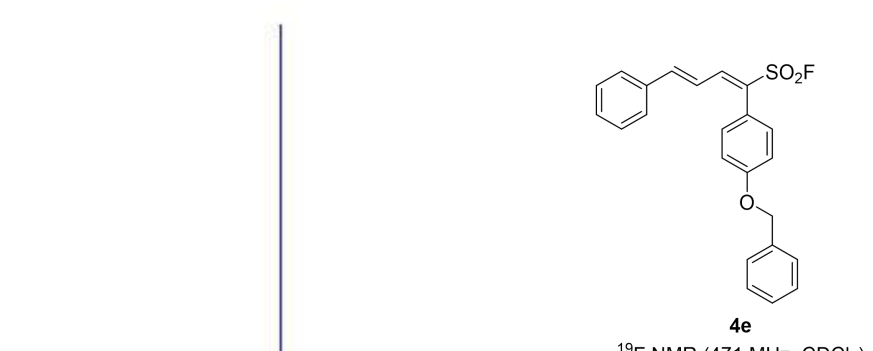

${ }^{19} \mathrm{~F} \mathrm{NMR}\left(471 \mathrm{MHz}, \mathrm{CDCl}_{3}\right)$

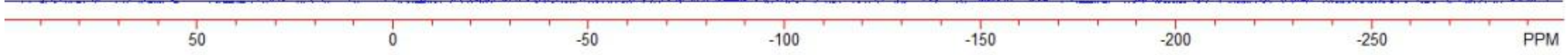




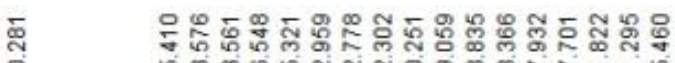

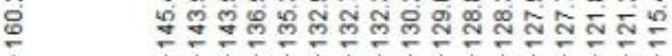

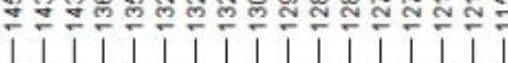

응

$\rightarrow \rightarrow 1 / l_{1}$
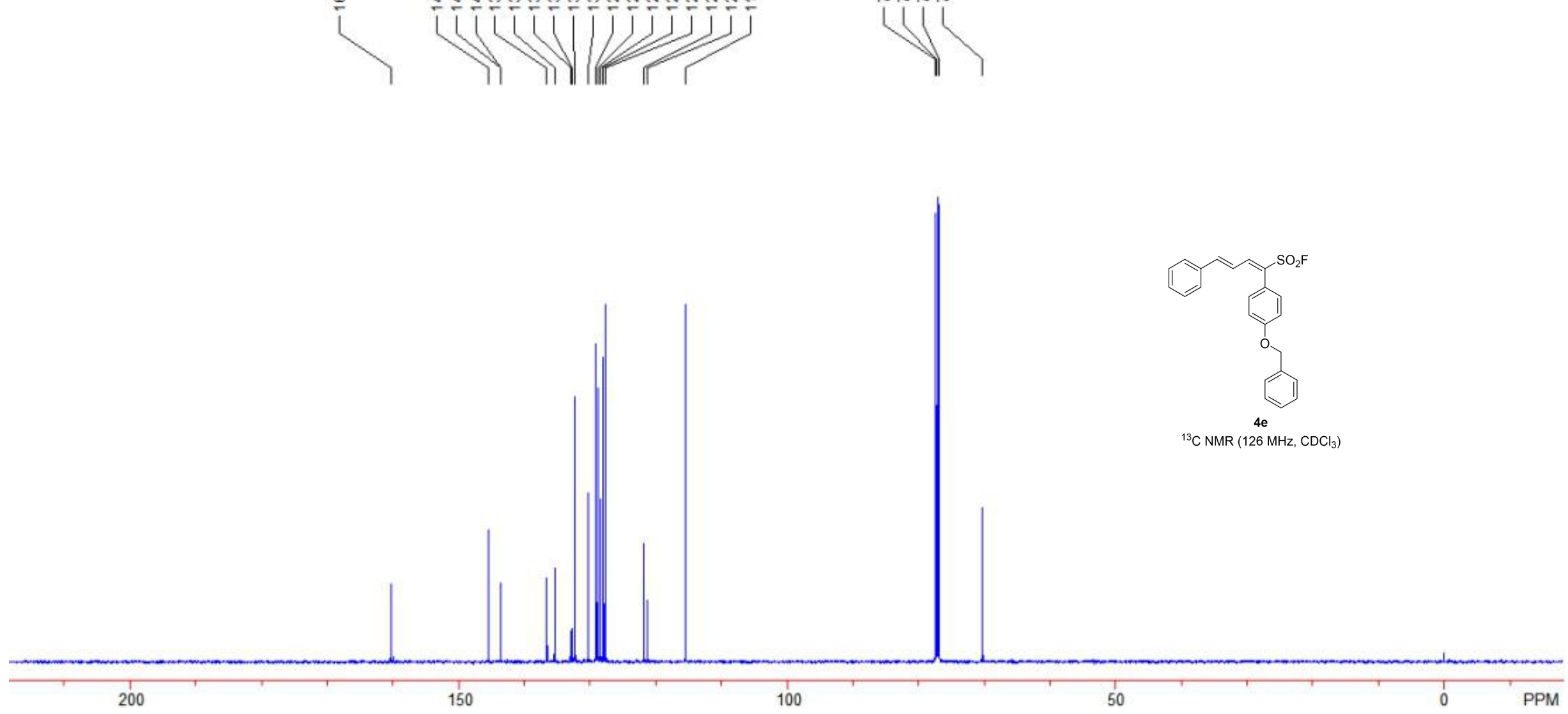


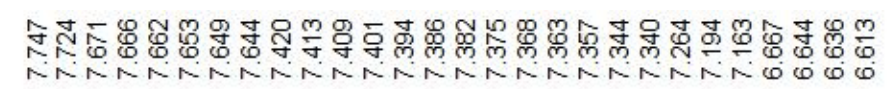

LLLLLLLLLLU|JjJJ」J」」」」」J

mit 1

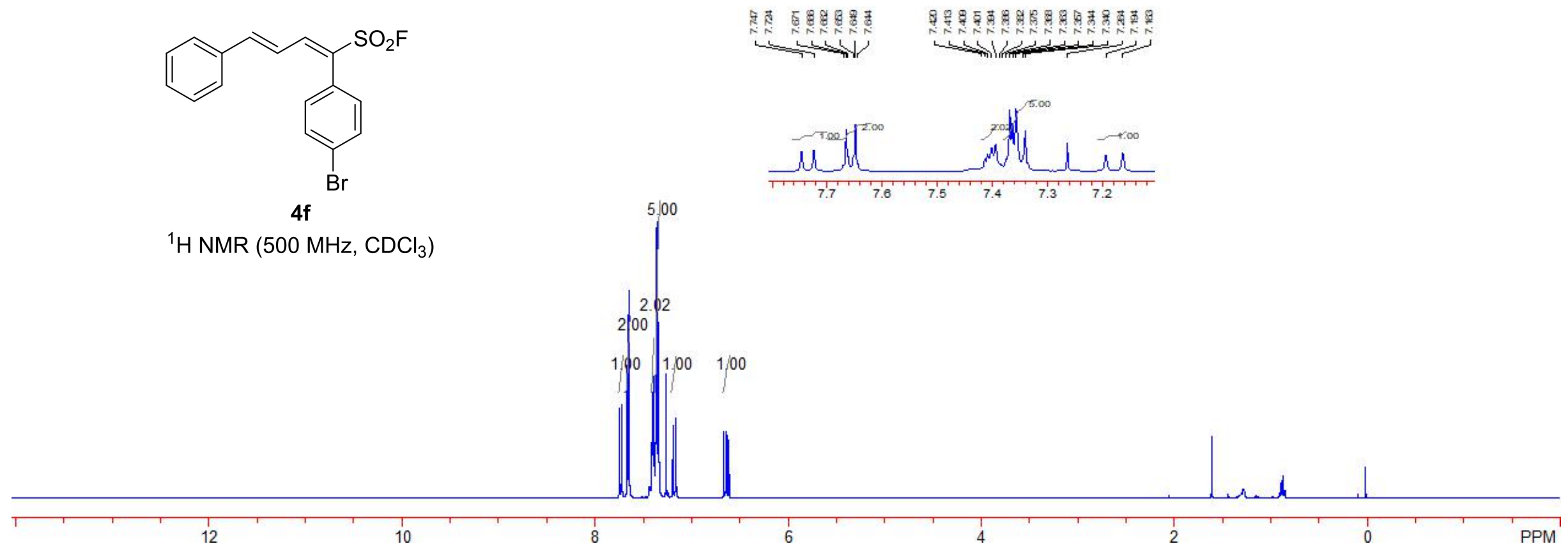



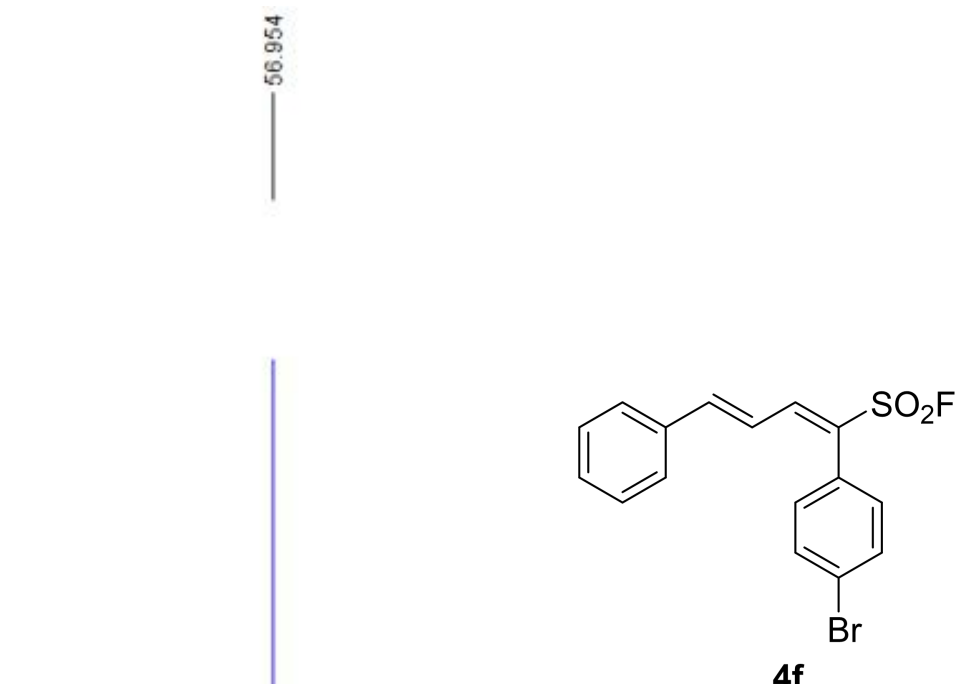

${ }^{19} \mathrm{~F} \mathrm{NMR}\left(471 \mathrm{MHz}, \mathrm{CDCl}_{3}\right)$ 


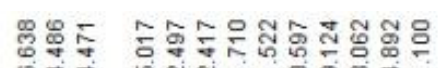

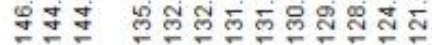

i广 i i ijjoj

1 mil

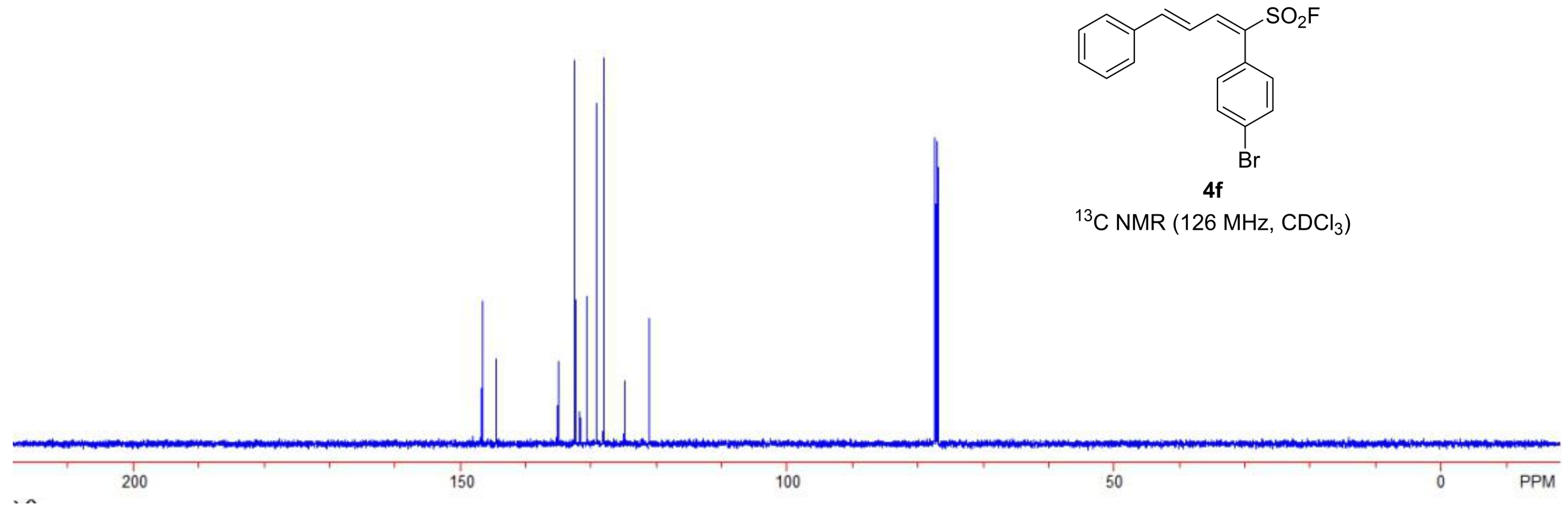




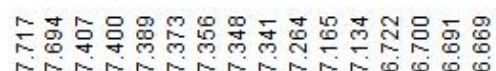

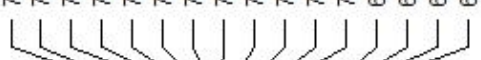

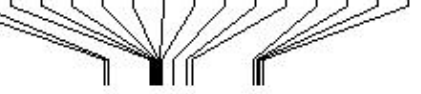
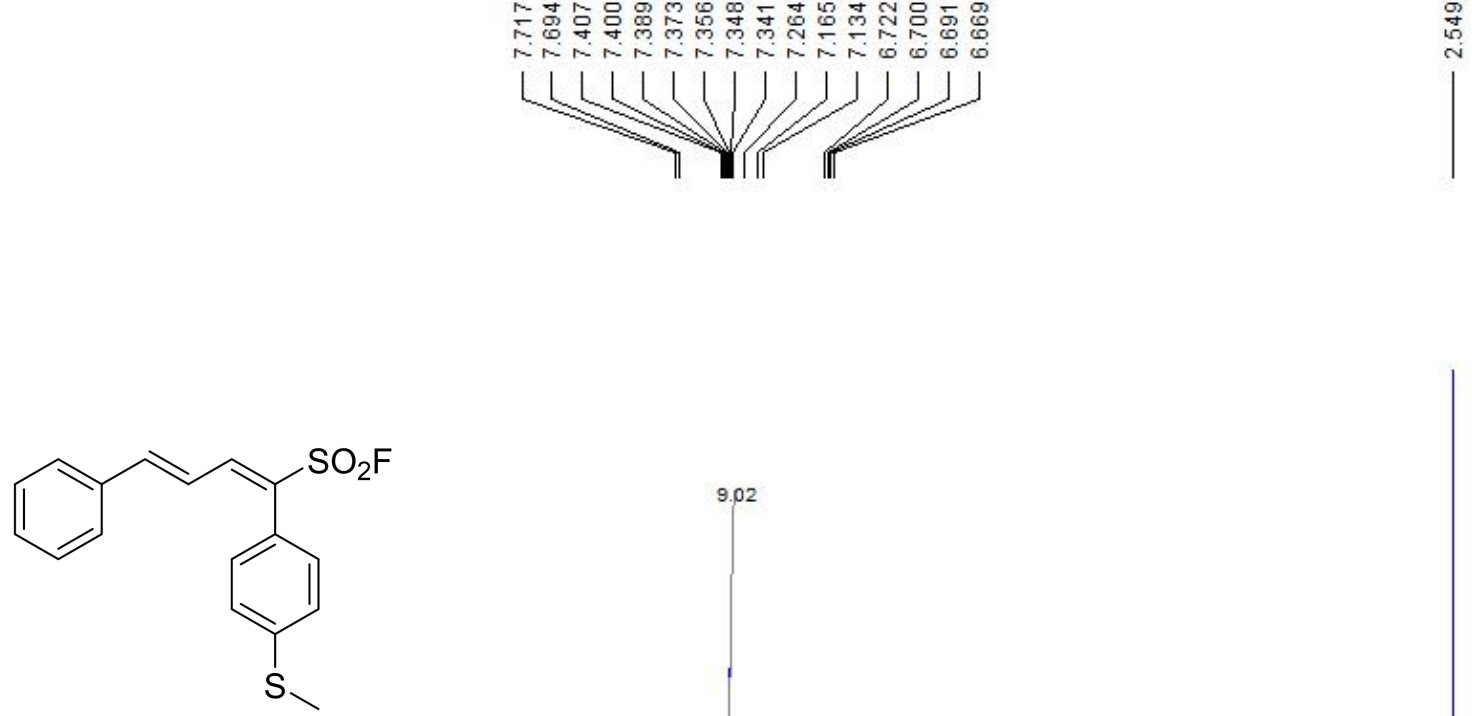

9.02

${ }^{1} \mathrm{H}$ NMR $\left(500 \mathrm{MHz}, \mathrm{CDCl}_{3}\right)$

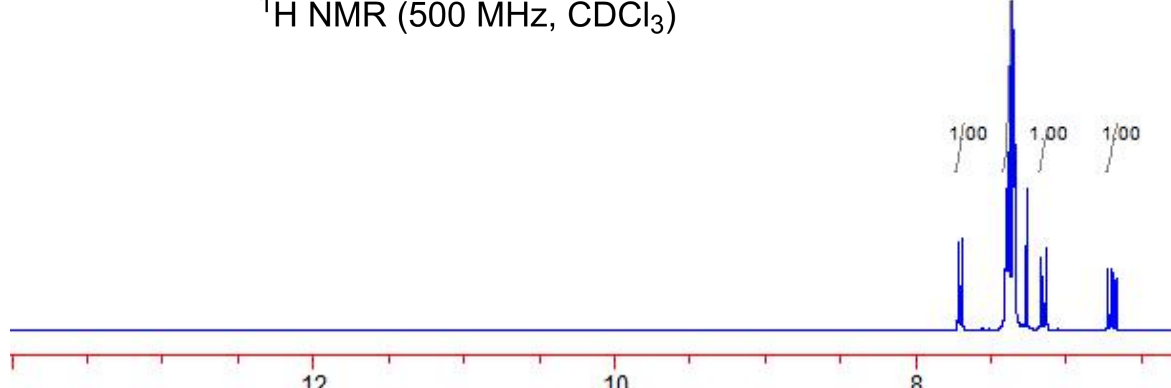

12

10

6

4

0 


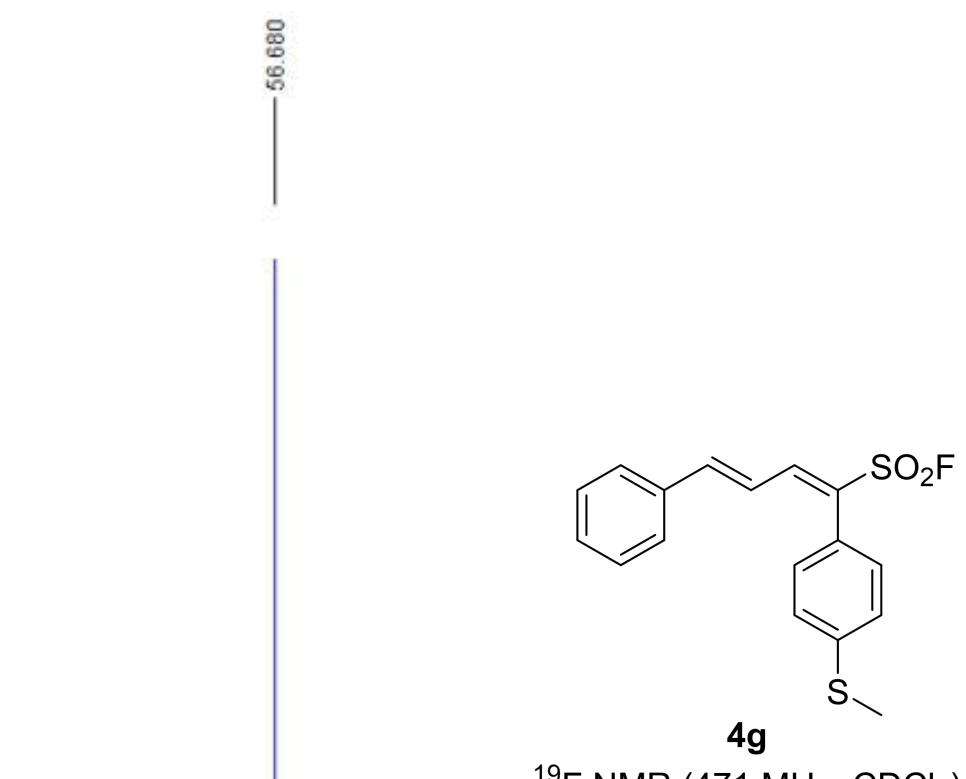

${ }^{19} \mathrm{~F} \mathrm{NMR}\left(471 \mathrm{MHz}, \mathrm{CDCl}_{3}\right)$ 

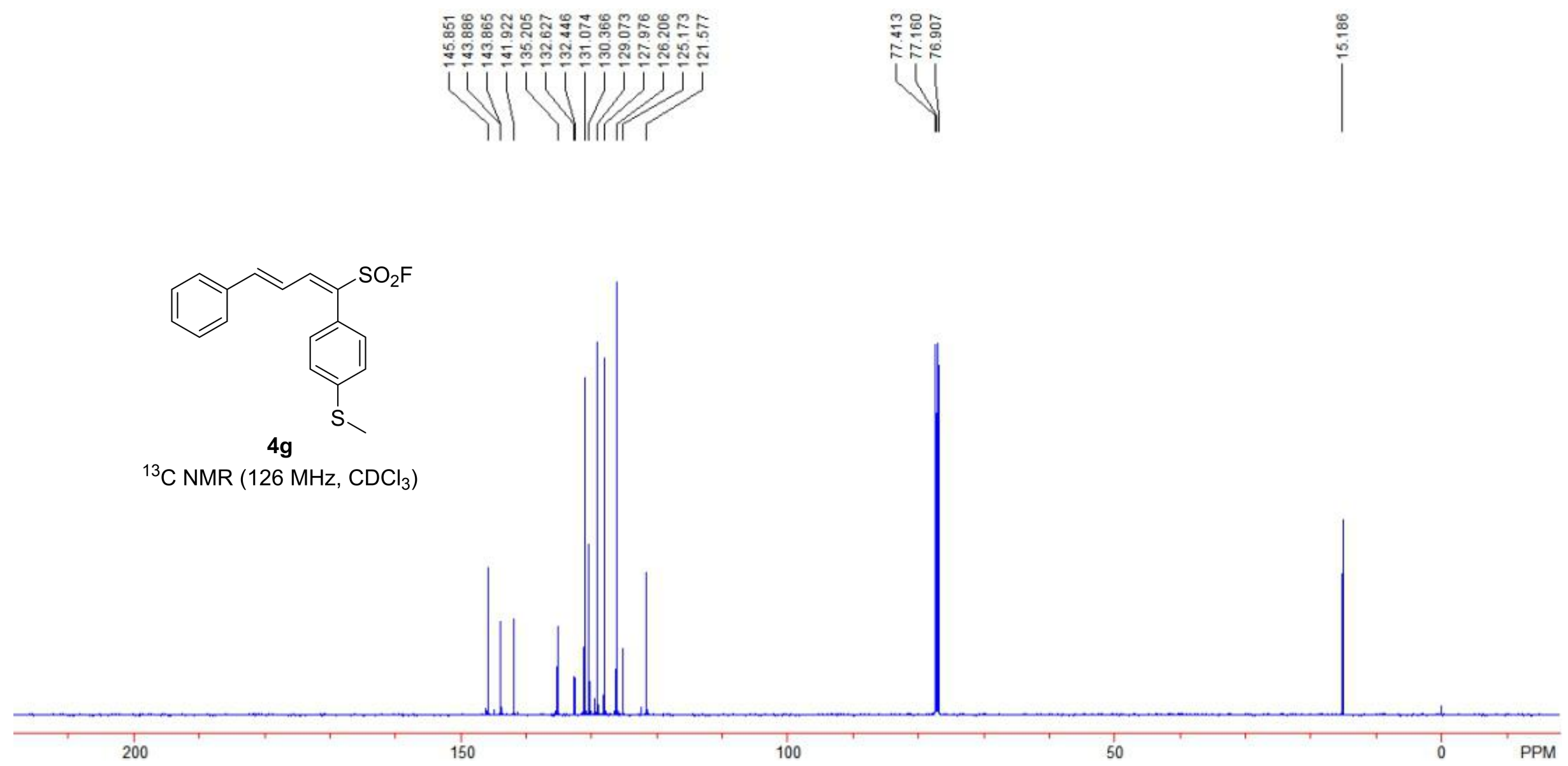


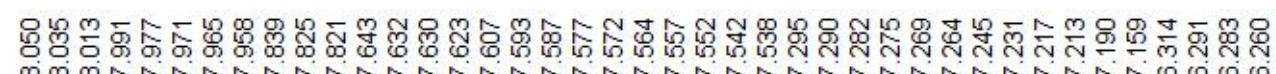

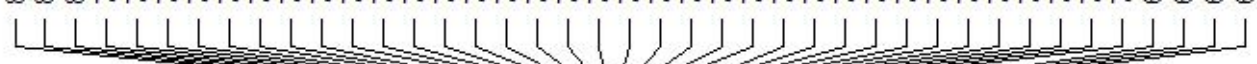

int III

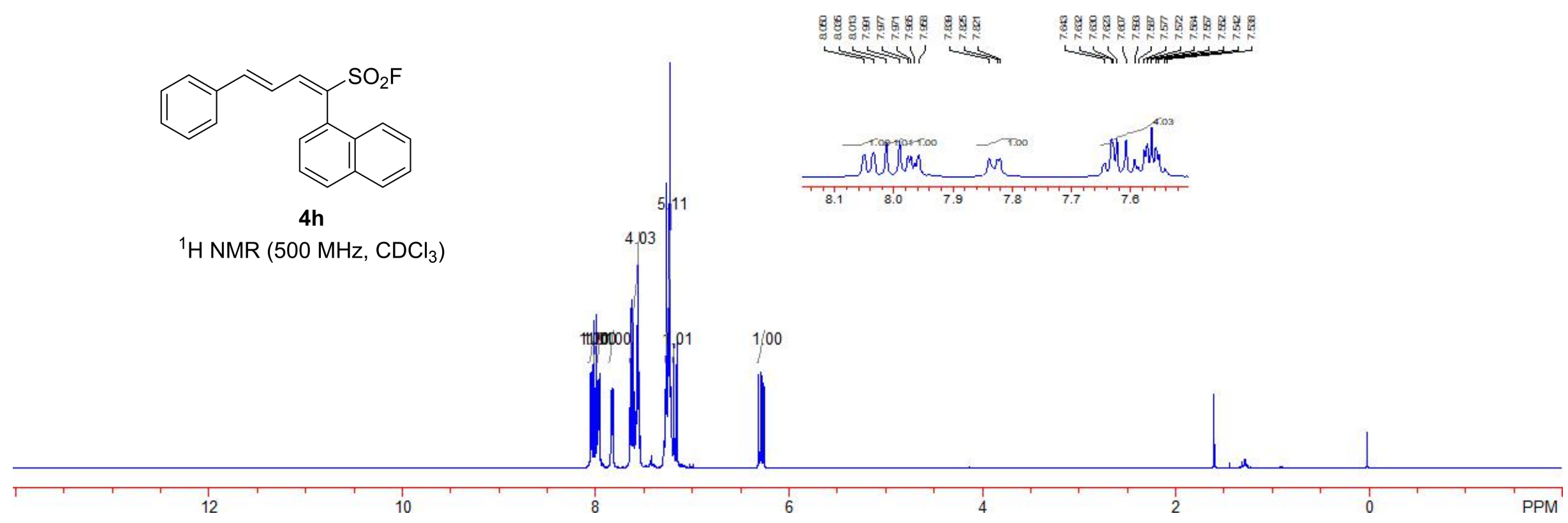




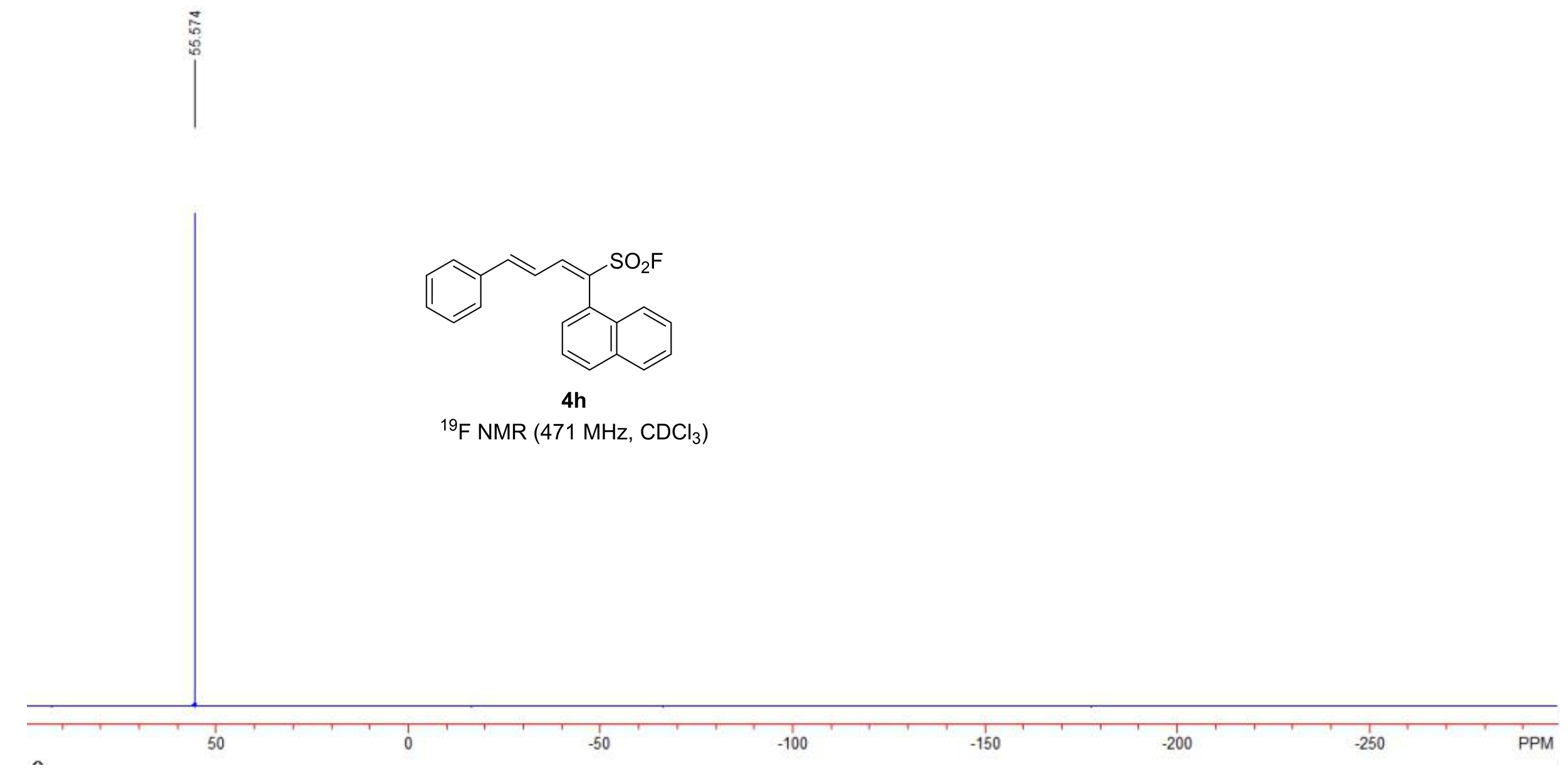



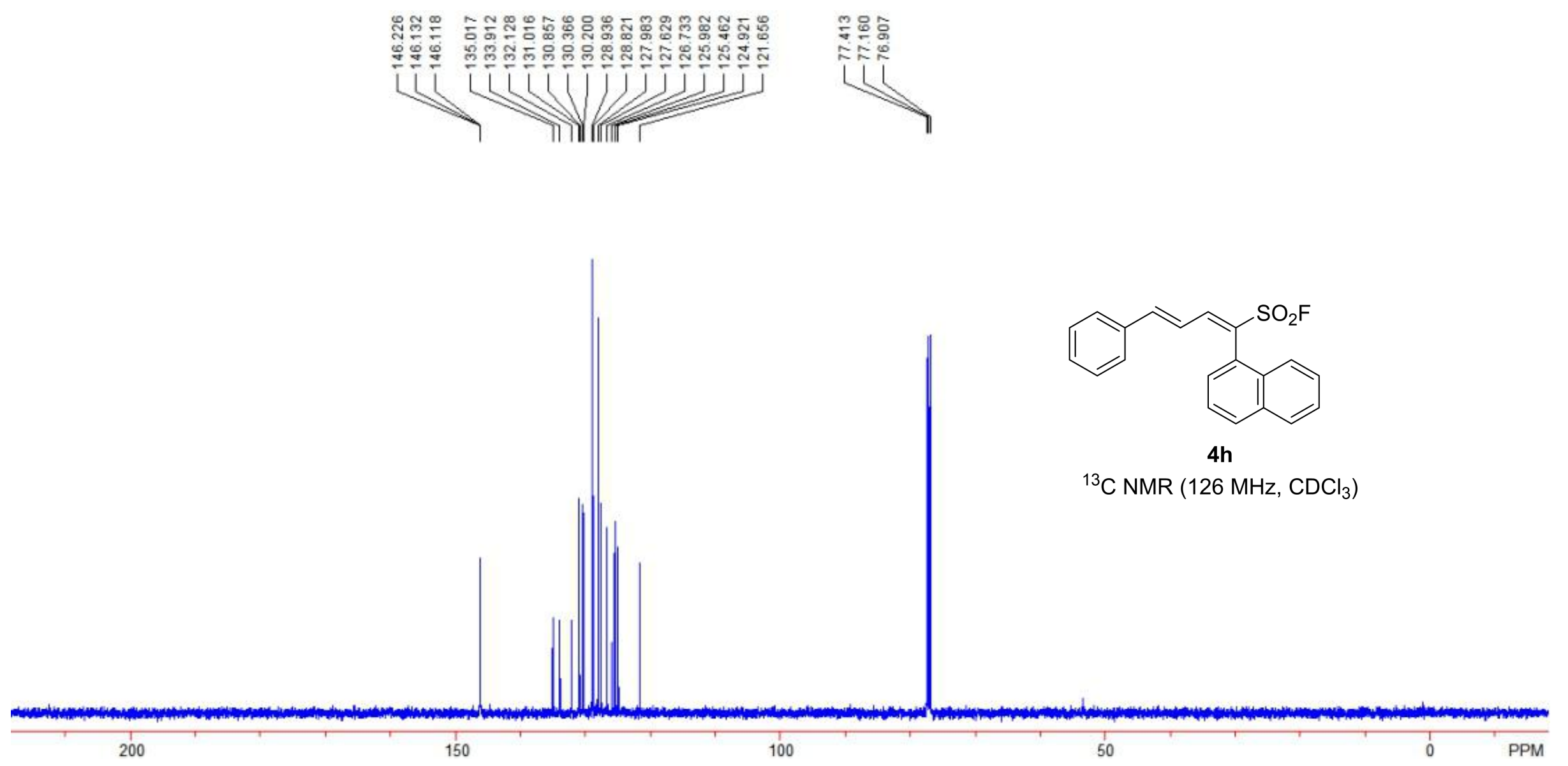


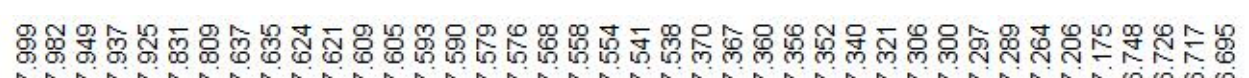

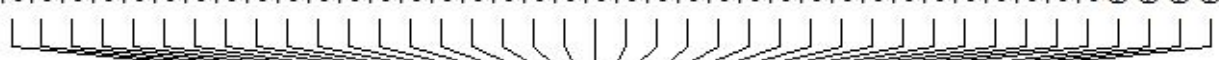

TाTा $\|$ III $\pi$
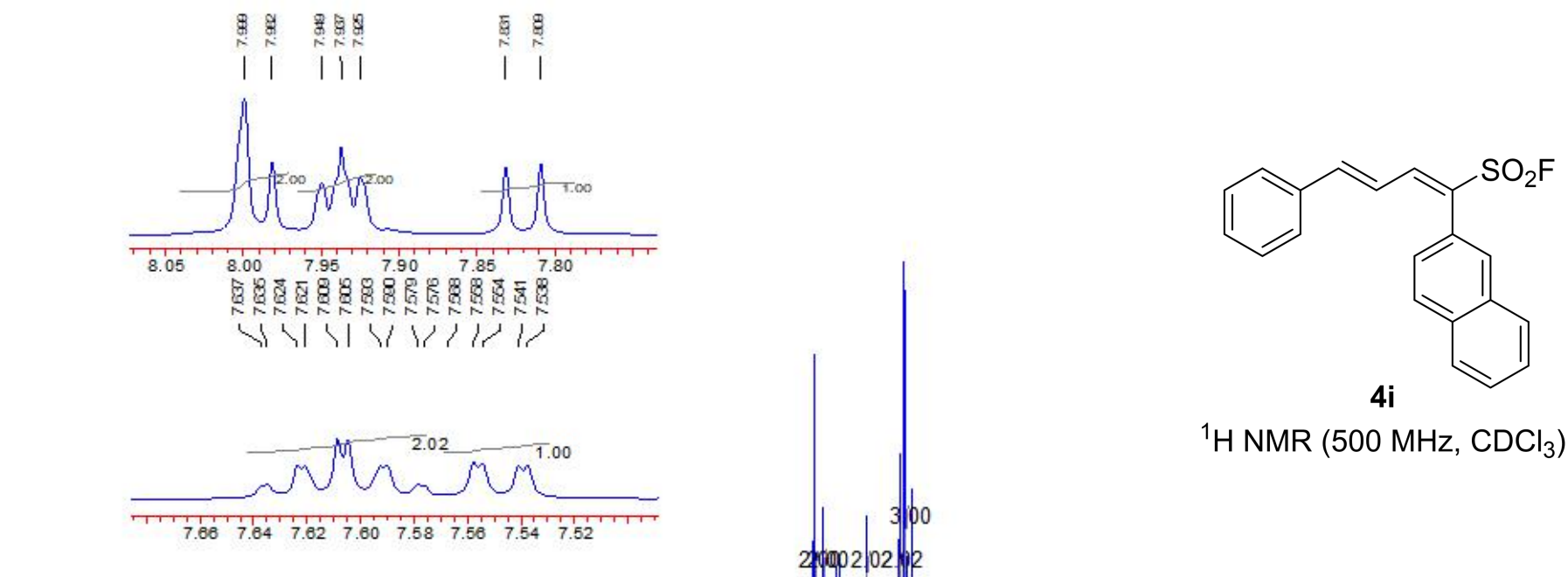

${ }^{1} \mathrm{H}$ NMR $\left(500 \mathrm{MHz}, \mathrm{CDCl}_{3}\right)$

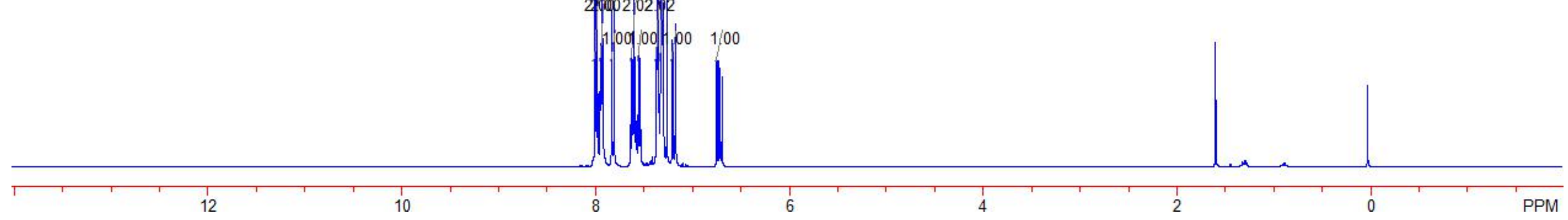




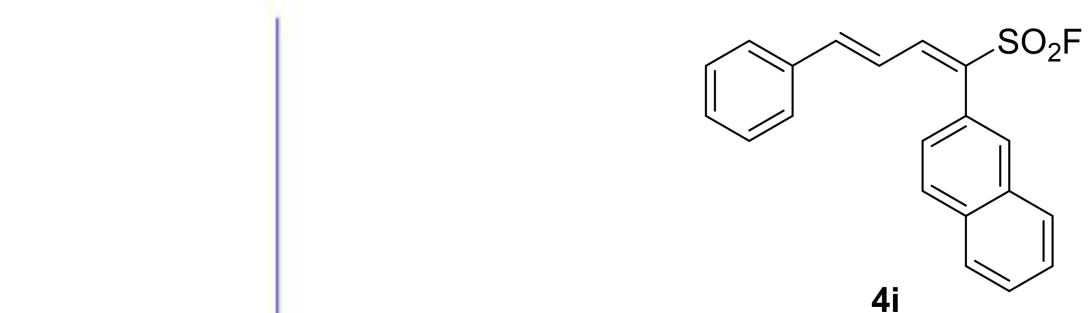

${ }^{19} \mathrm{~F} \mathrm{NMR}\left(471 \mathrm{MHz}, \mathrm{CDCl}_{3}\right)$

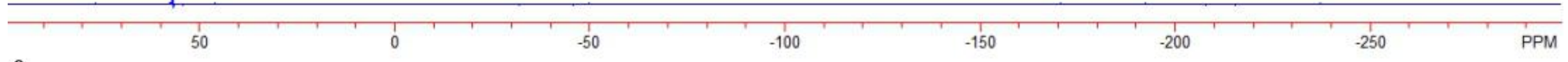




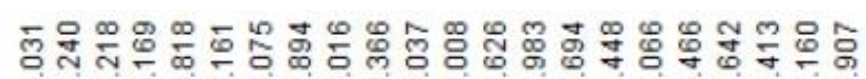

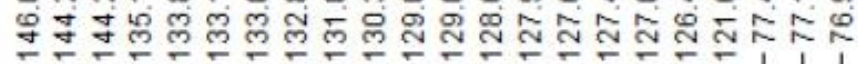

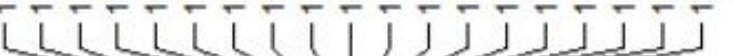

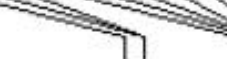
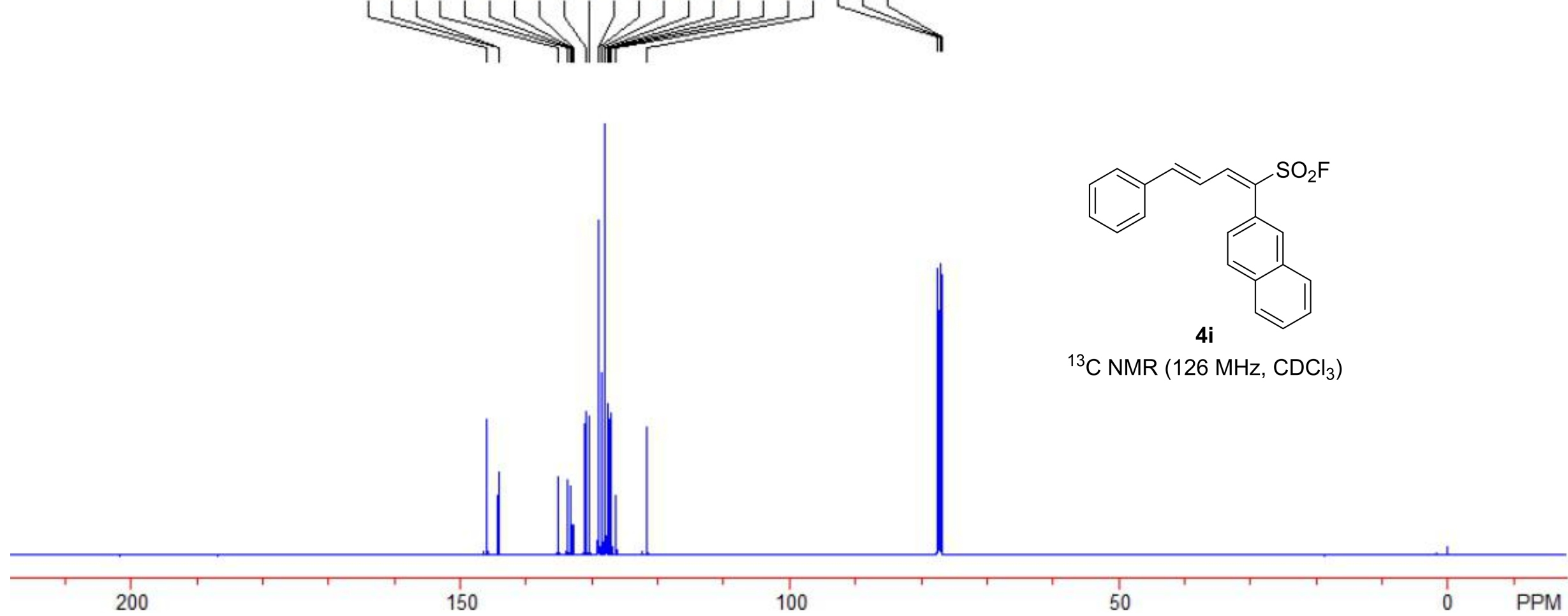


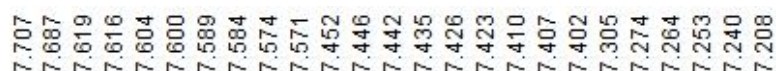

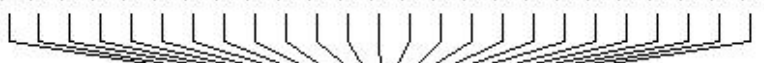

28. 711111

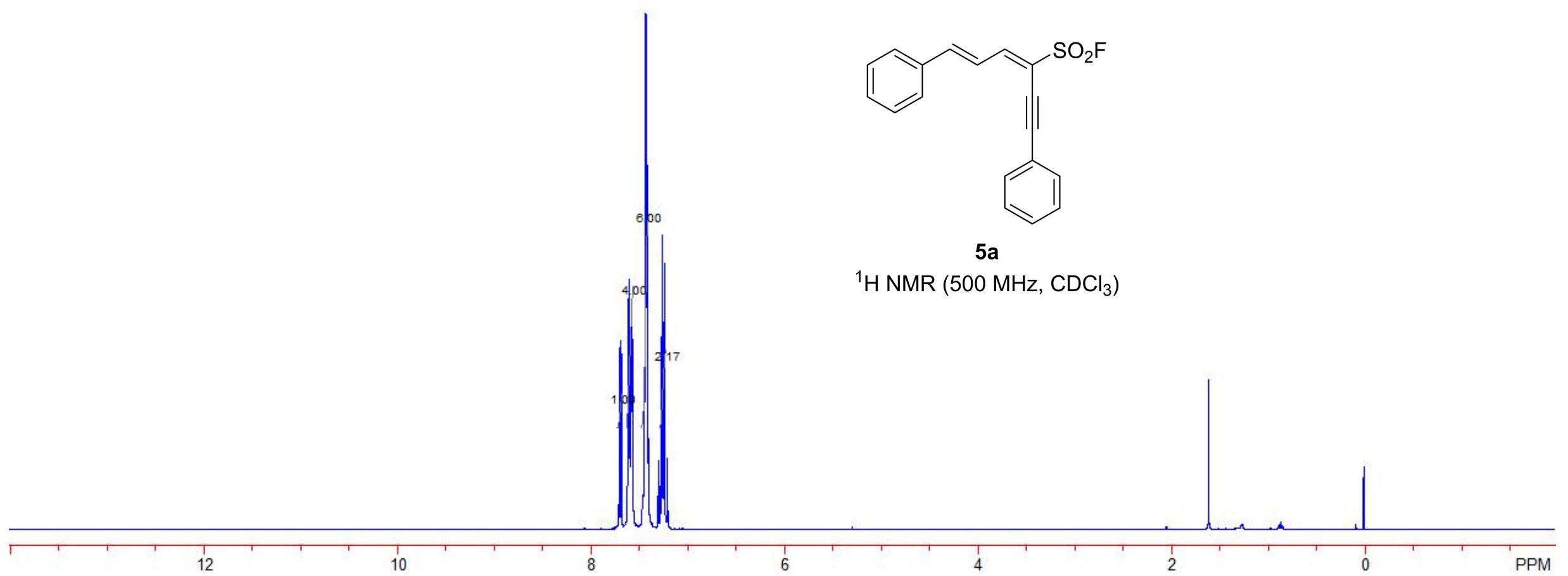




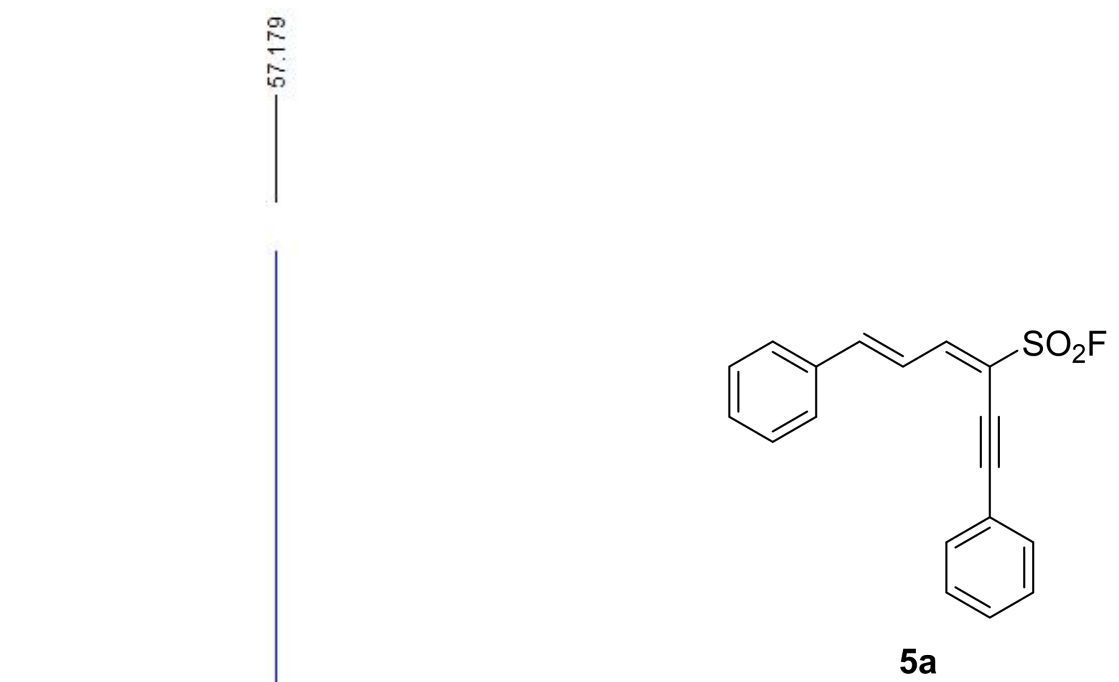

${ }^{19} \mathrm{~F} \mathrm{NMR}\left(471 \mathrm{MHz}, \mathrm{CDCl}_{3}\right)$ 

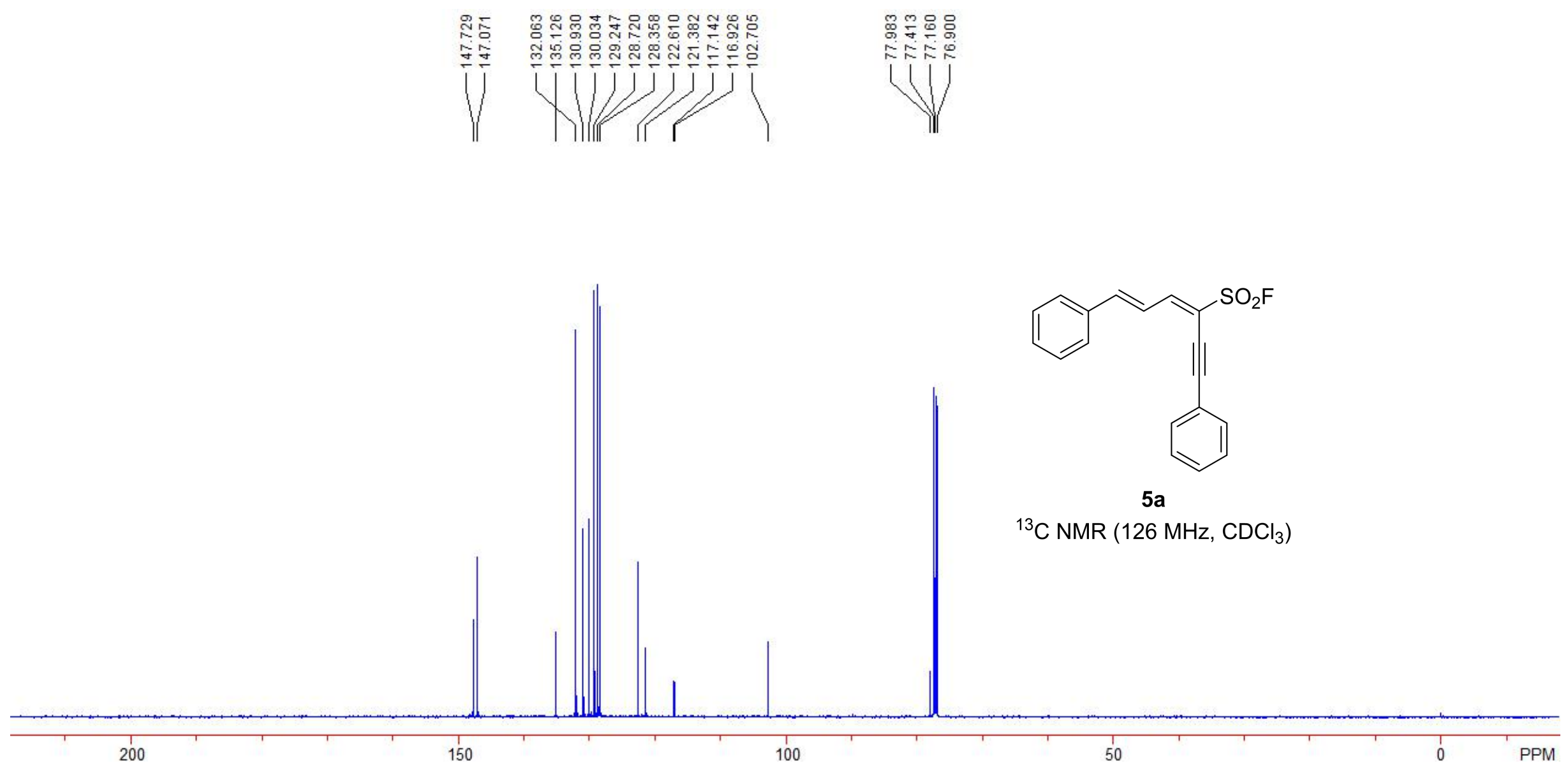


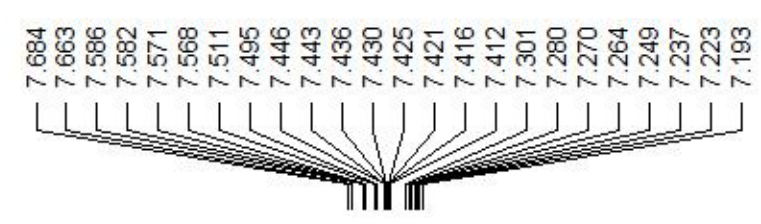

సั่
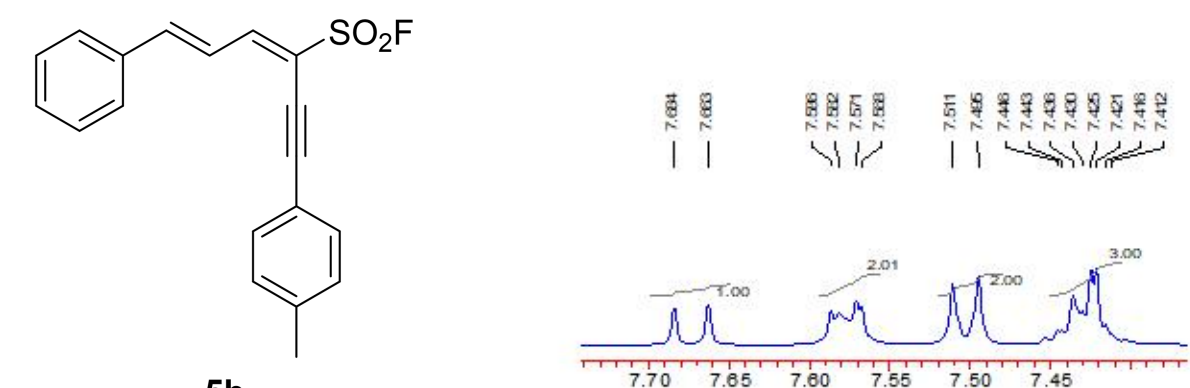

$5 b$

${ }^{1} \mathrm{H}$ NMR $\left(500 \mathrm{MHz}, \mathrm{CDCl}_{3}\right)$

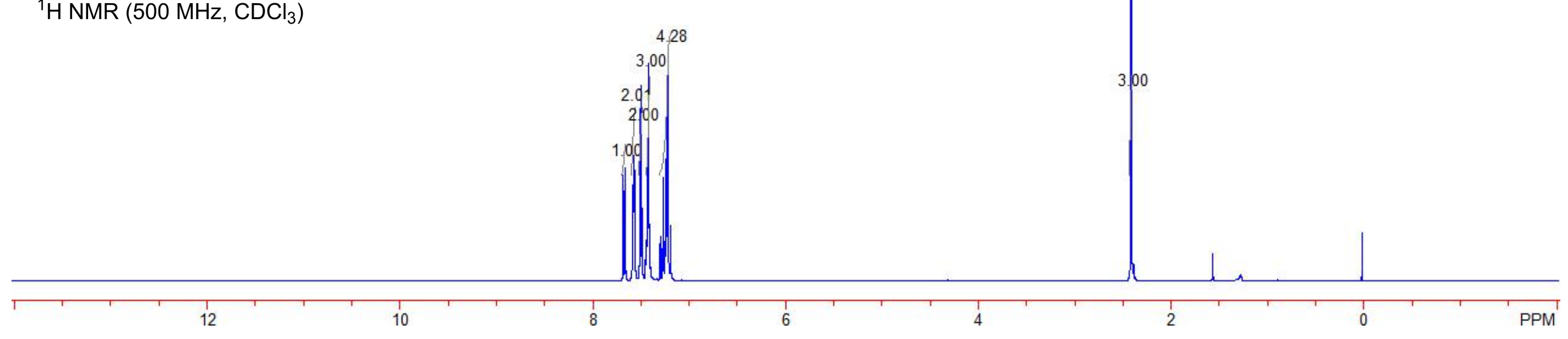




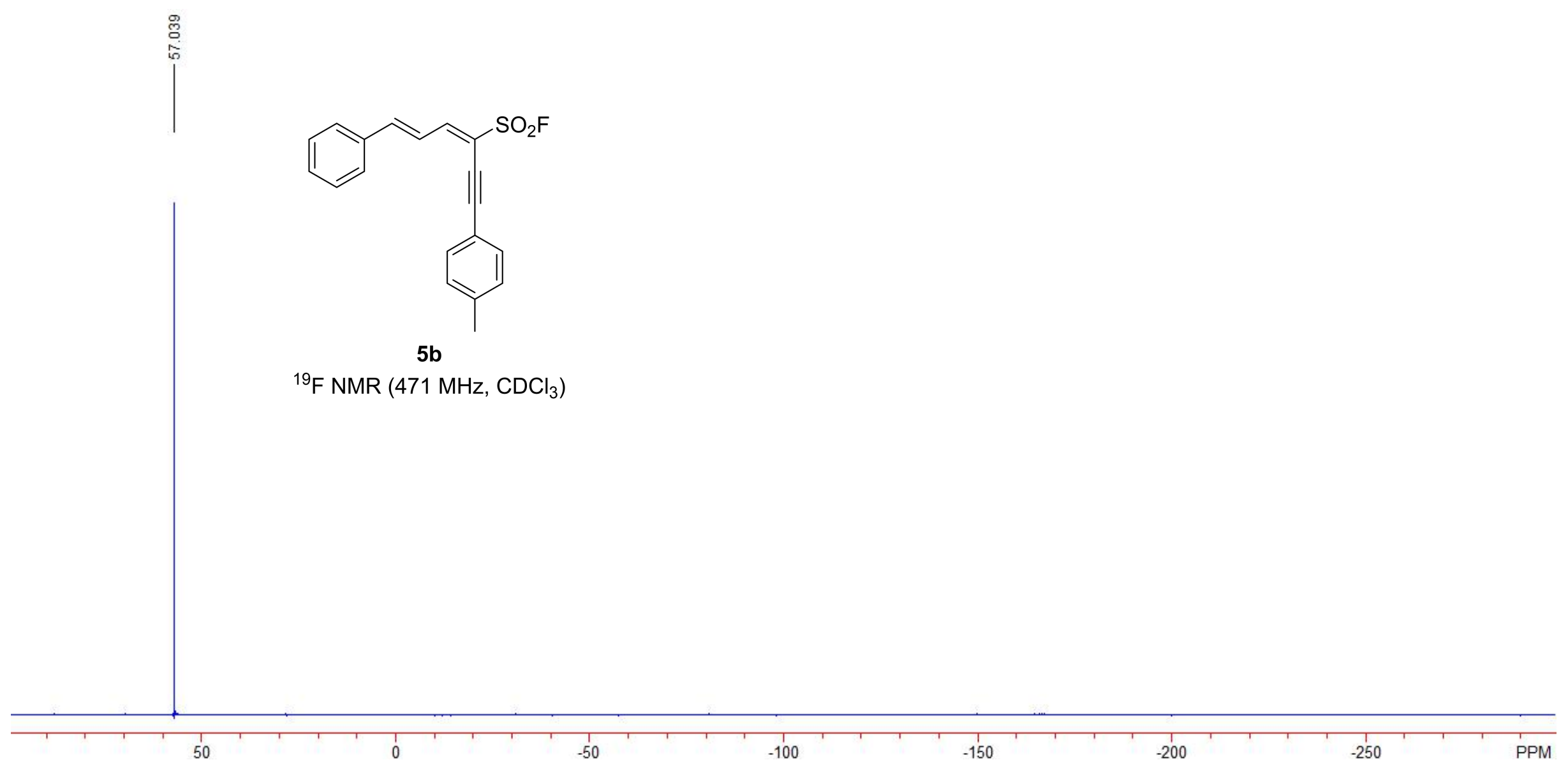



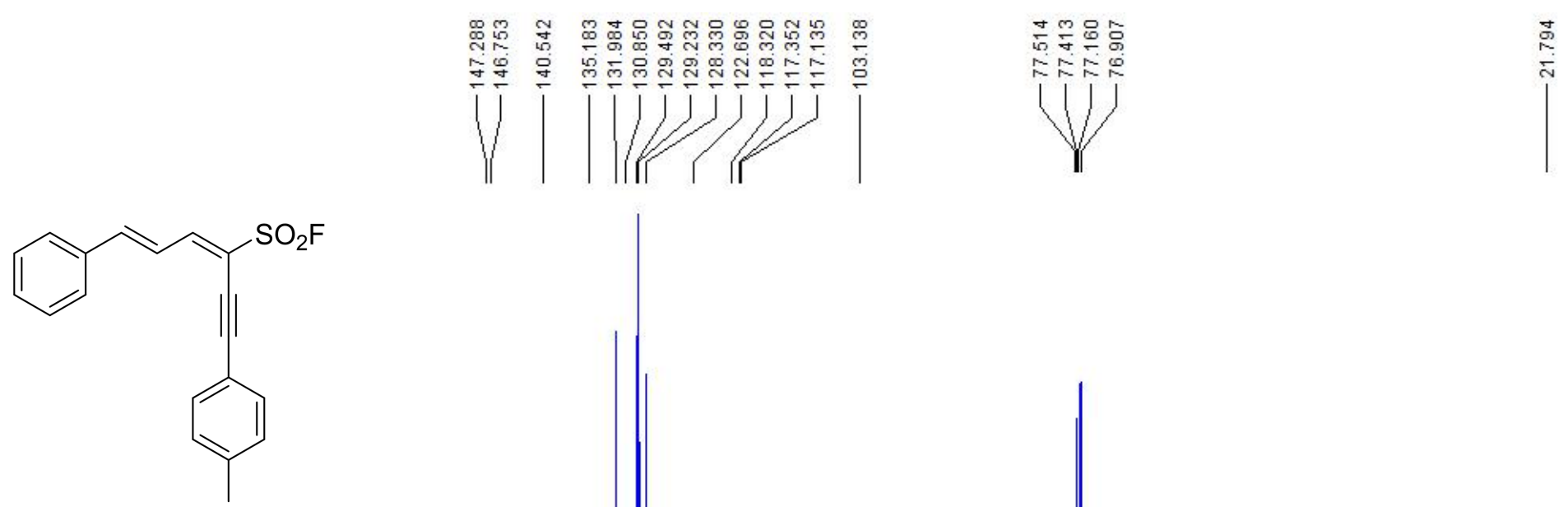

5b

${ }^{13} \mathrm{C}$ NMR $\left(126 \mathrm{MHz}, \mathrm{CDCl}_{3}\right)$

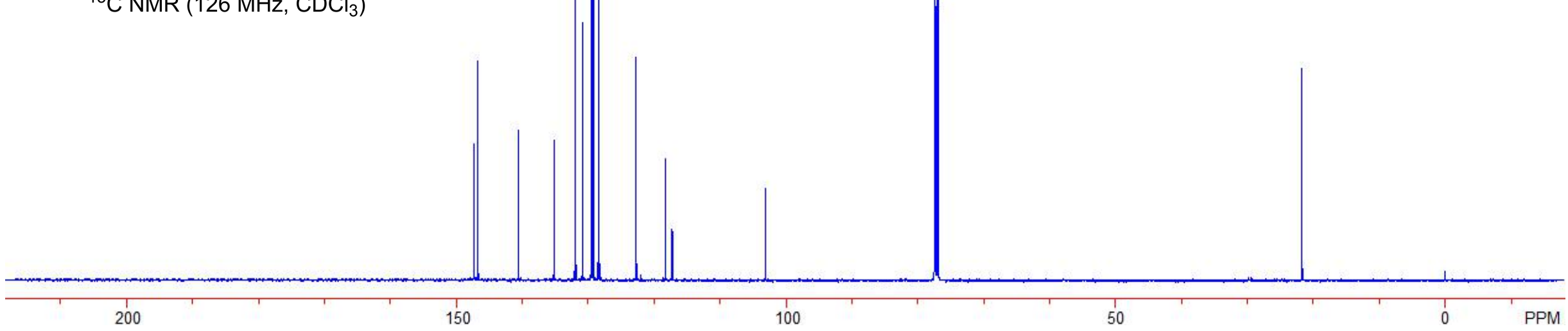




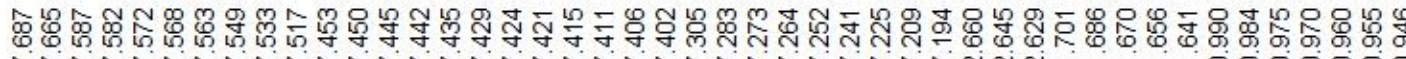

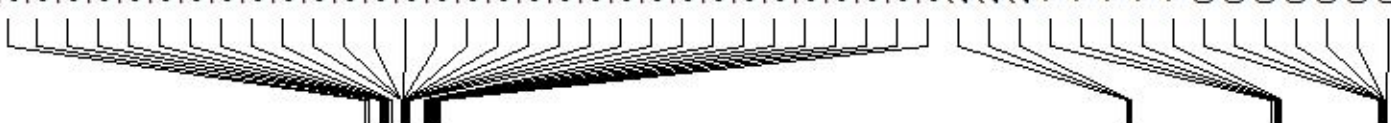

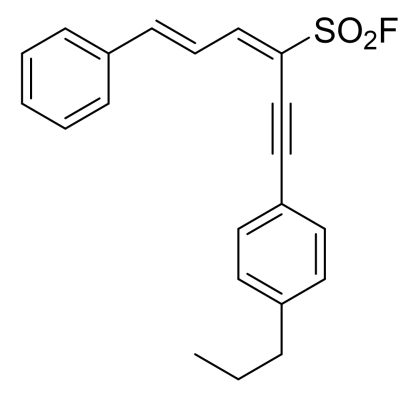

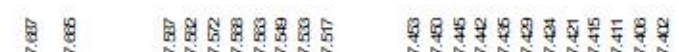

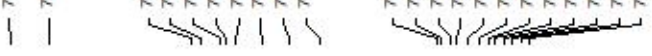

$5 \mathrm{c}$

${ }^{1} \mathrm{H} \mathrm{NMR}\left(500 \mathrm{MHz}, \mathrm{CDCl}_{3}\right)$
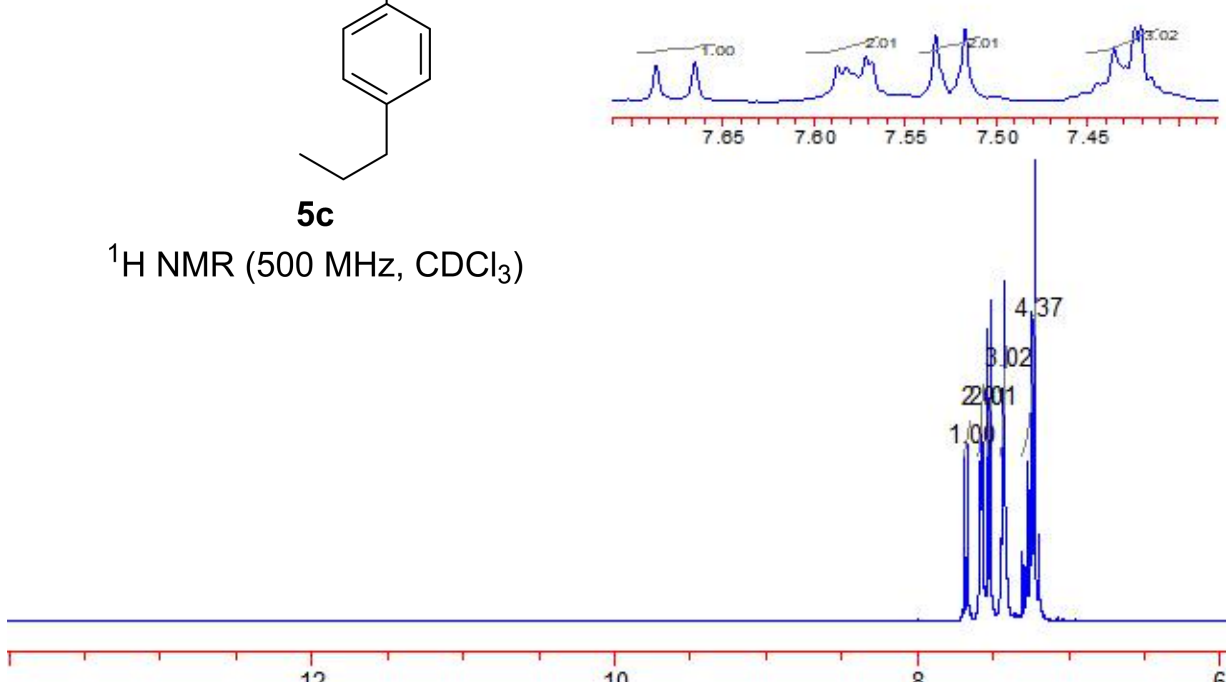

6 


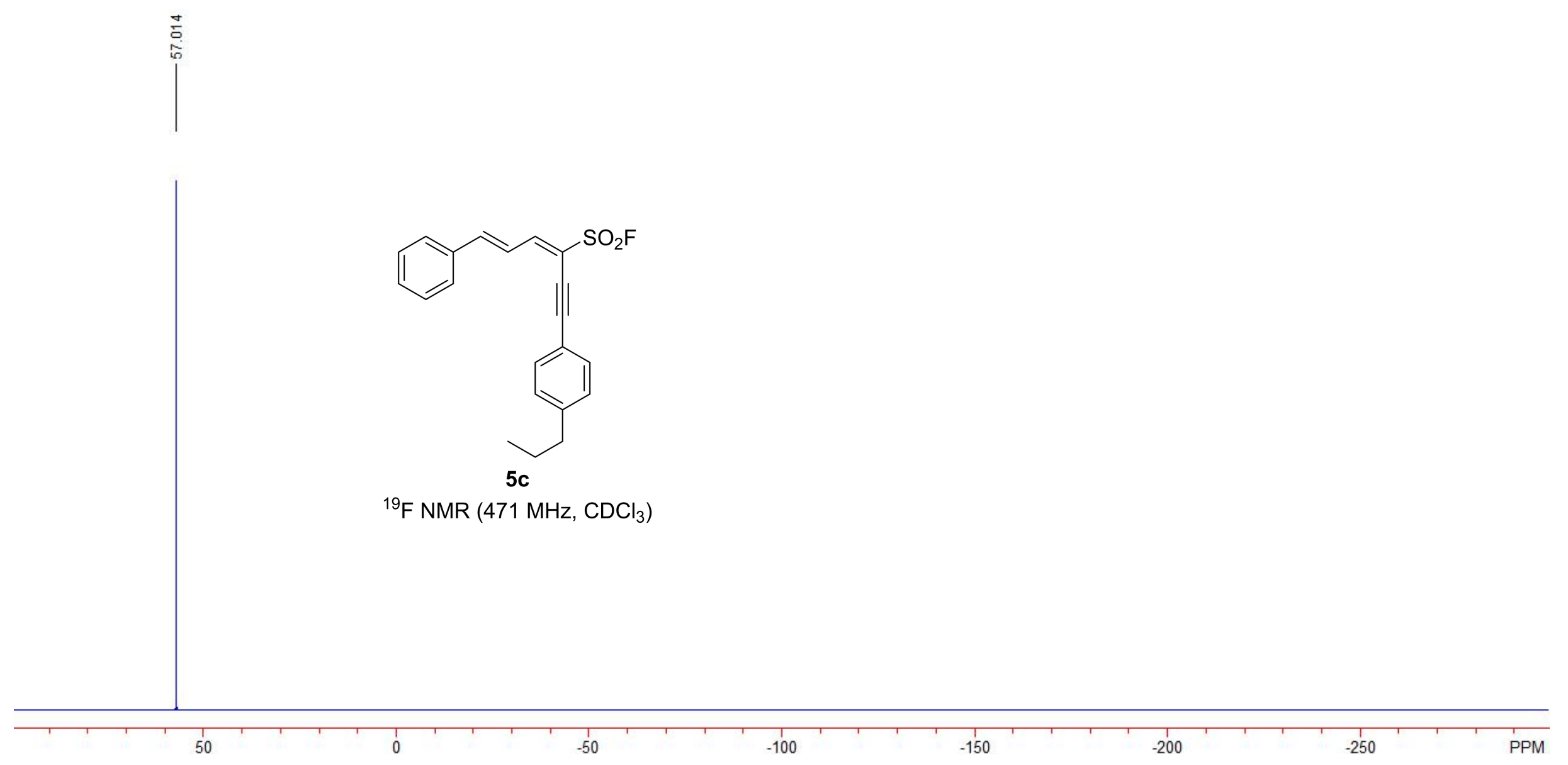



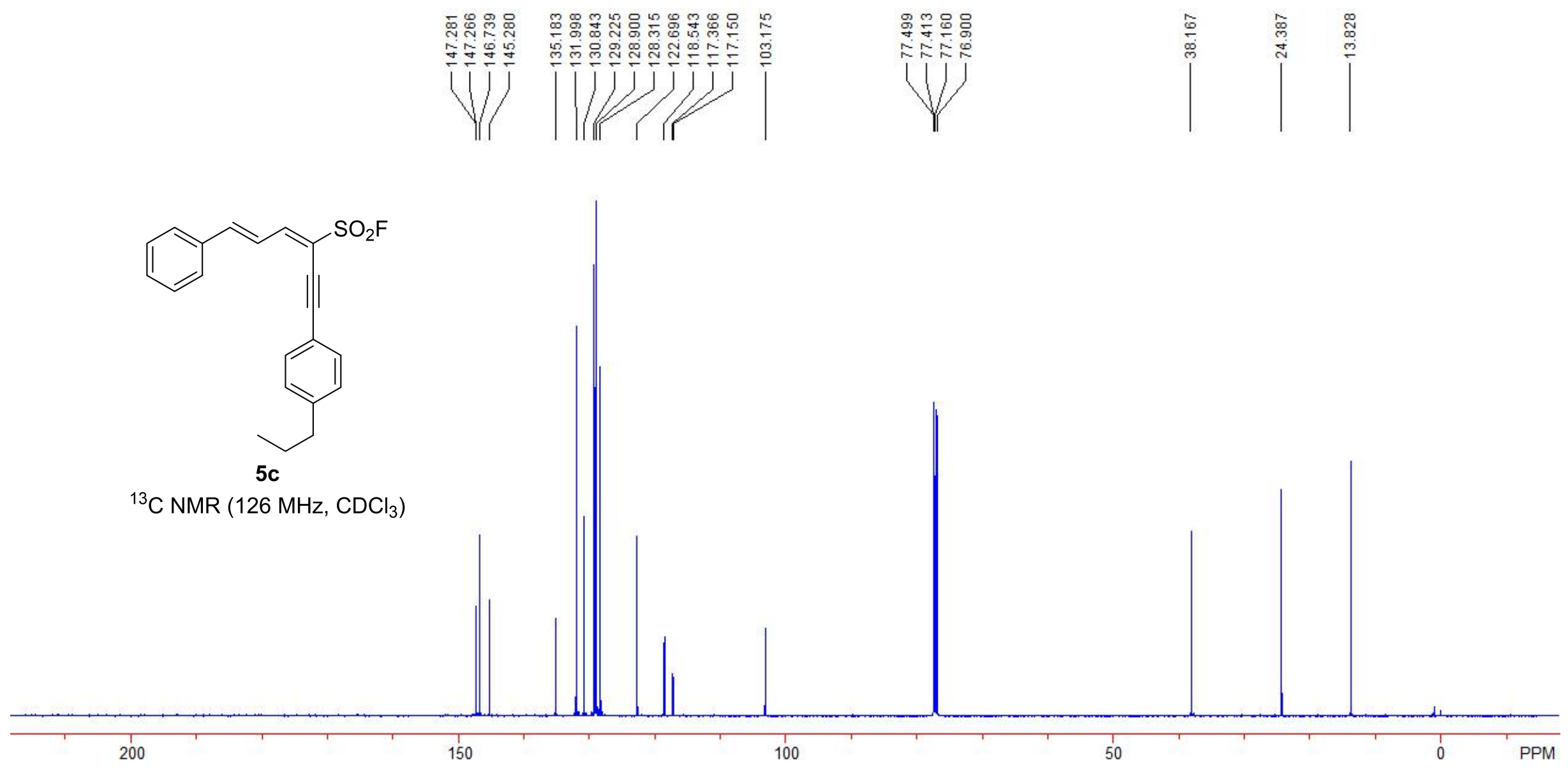


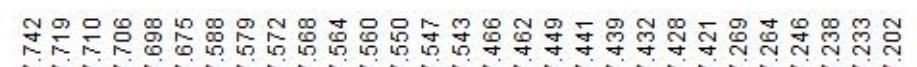

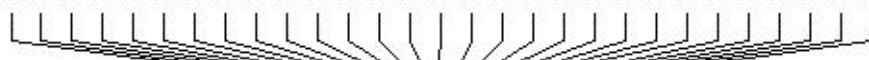

ता

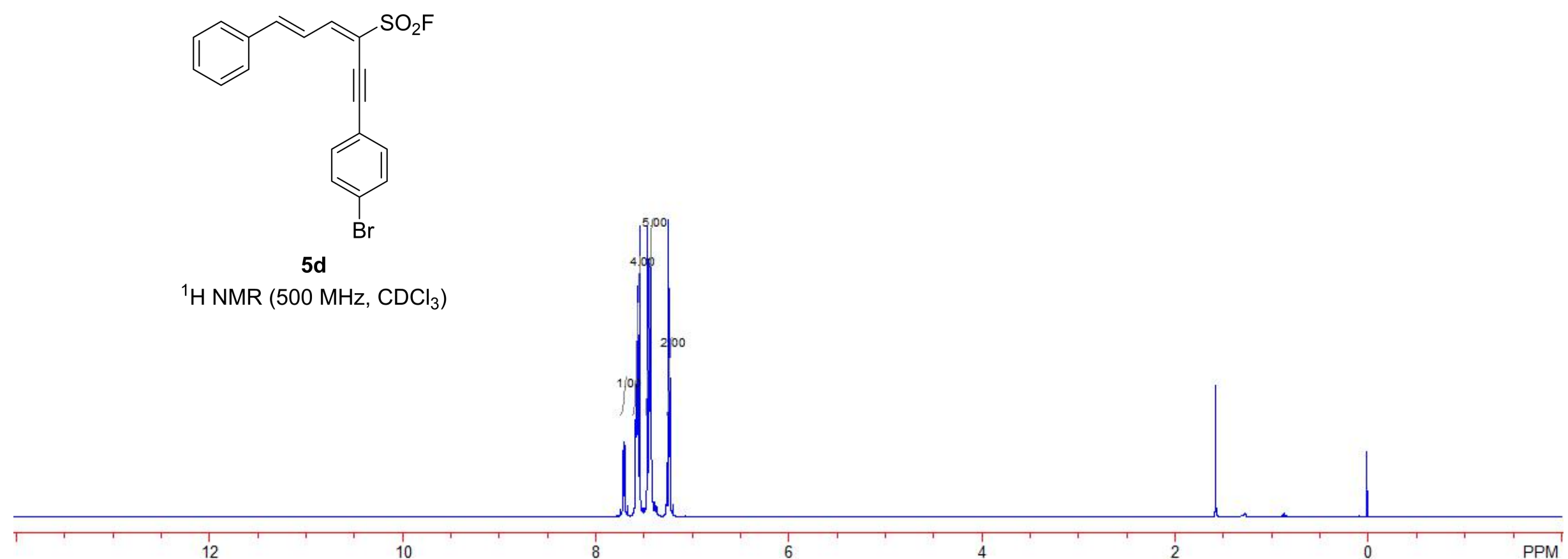




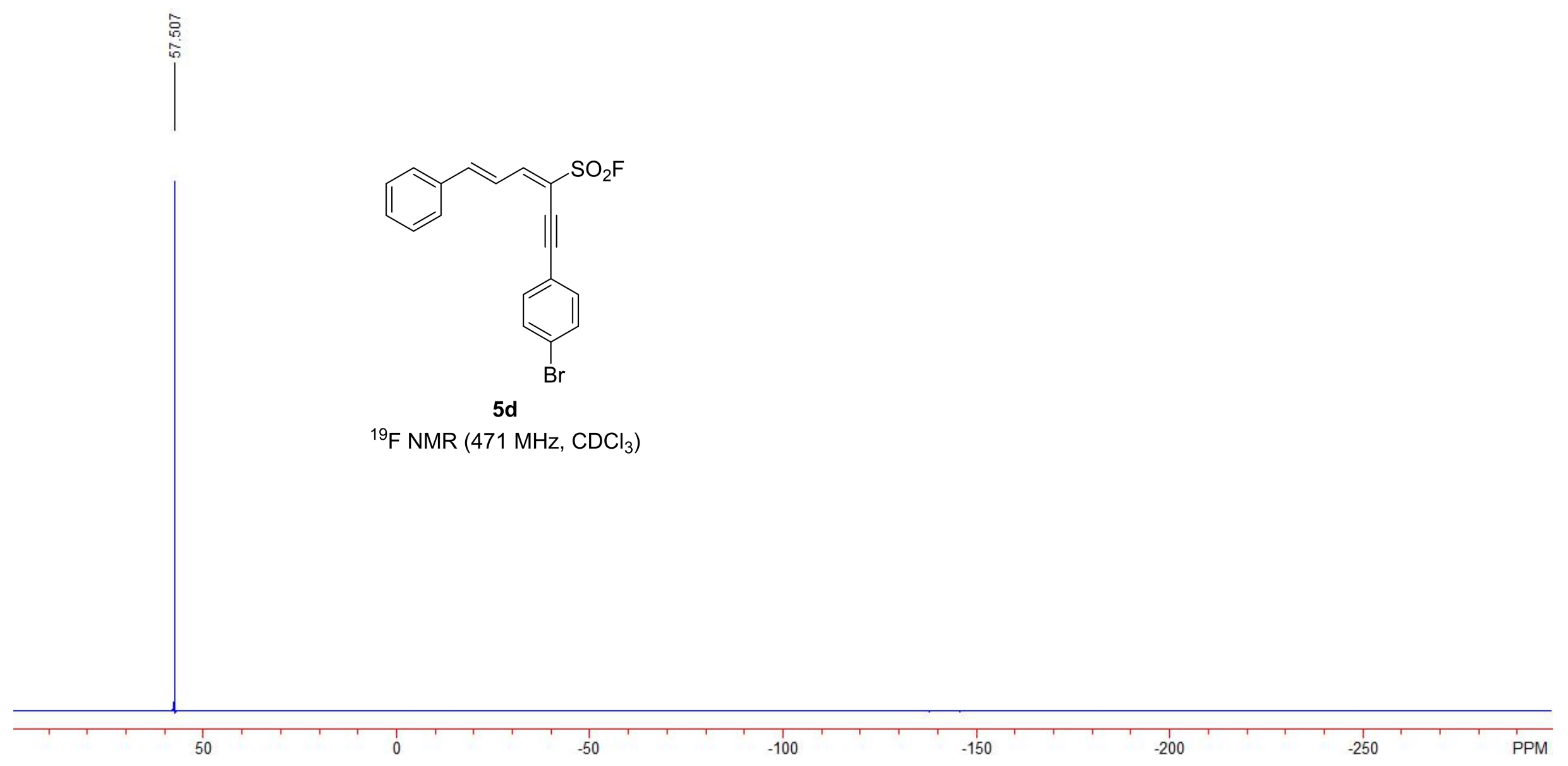




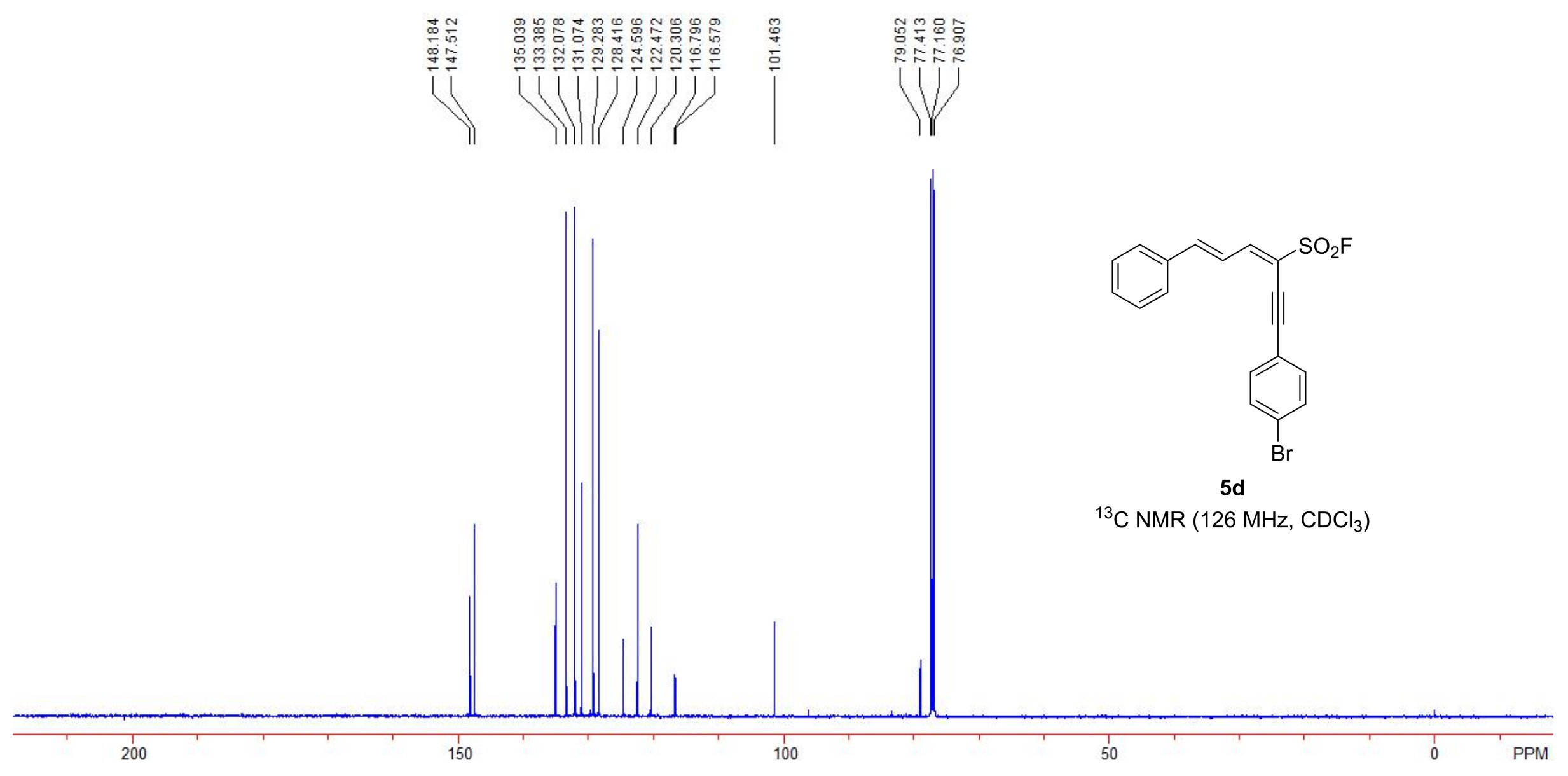




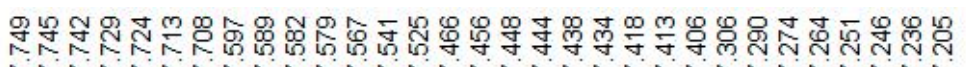

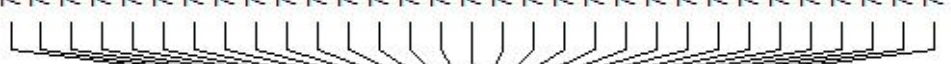

TIIIII

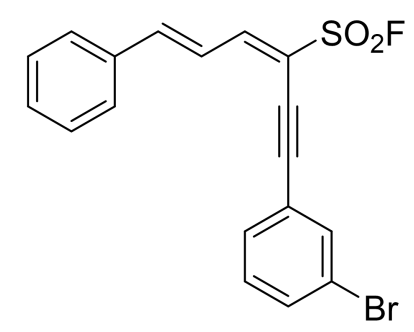

5 e

${ }^{1} \mathrm{H} \mathrm{NMR}\left(500 \mathrm{MHz}, \mathrm{CDCl}_{3}\right)$

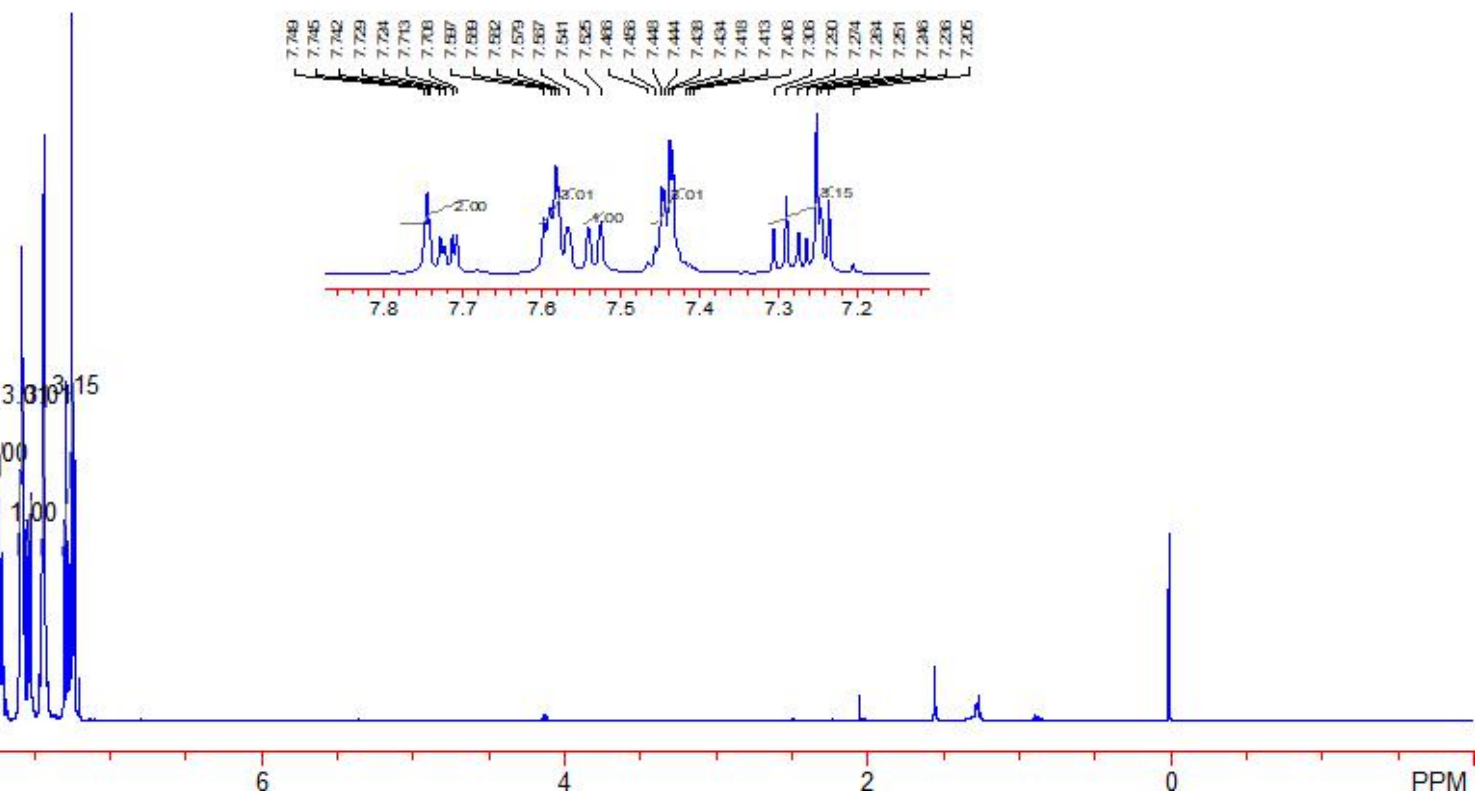




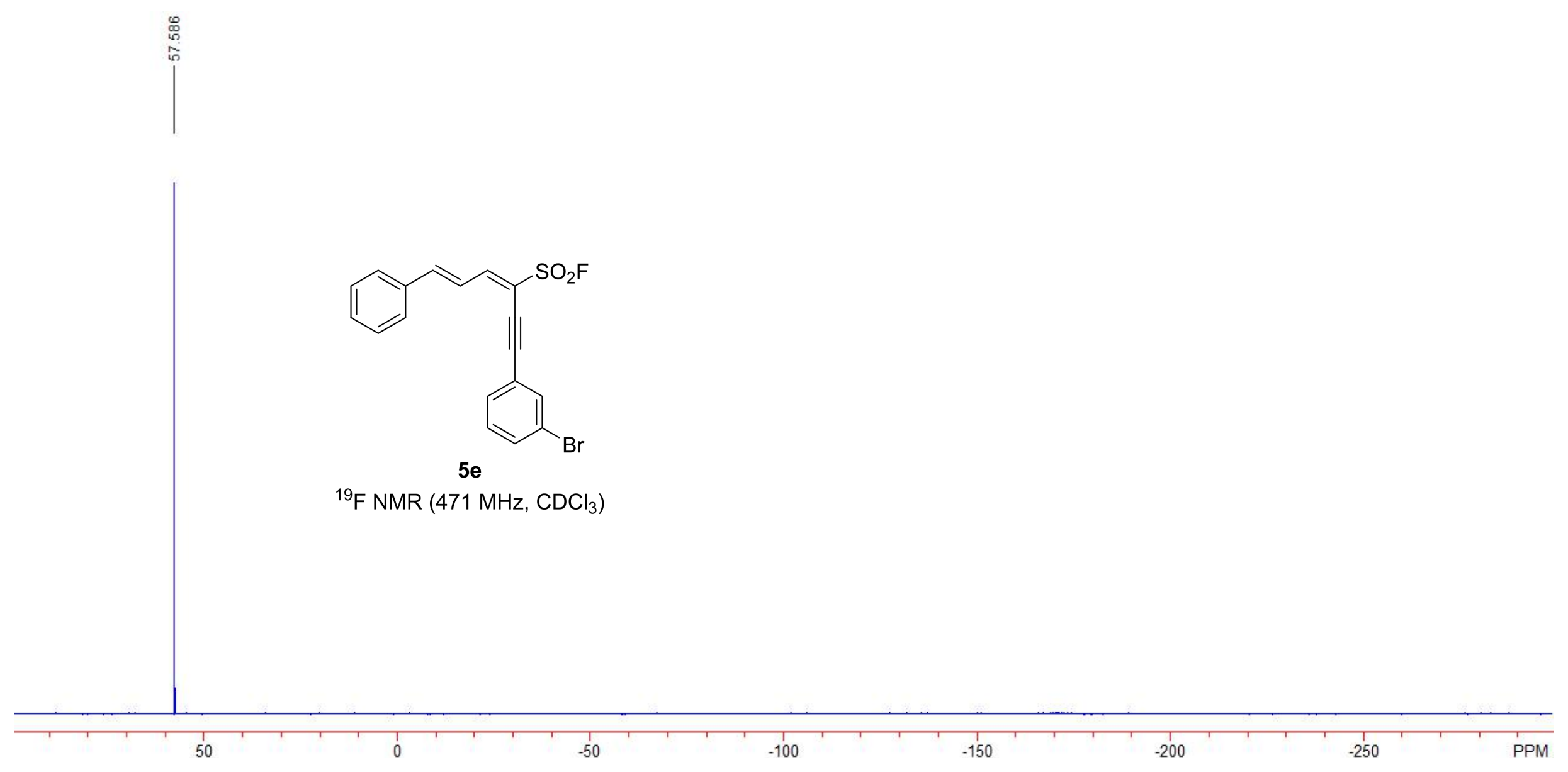




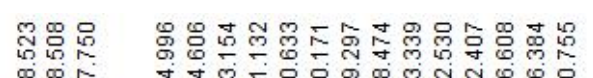

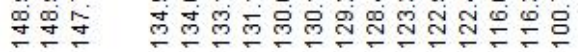

i

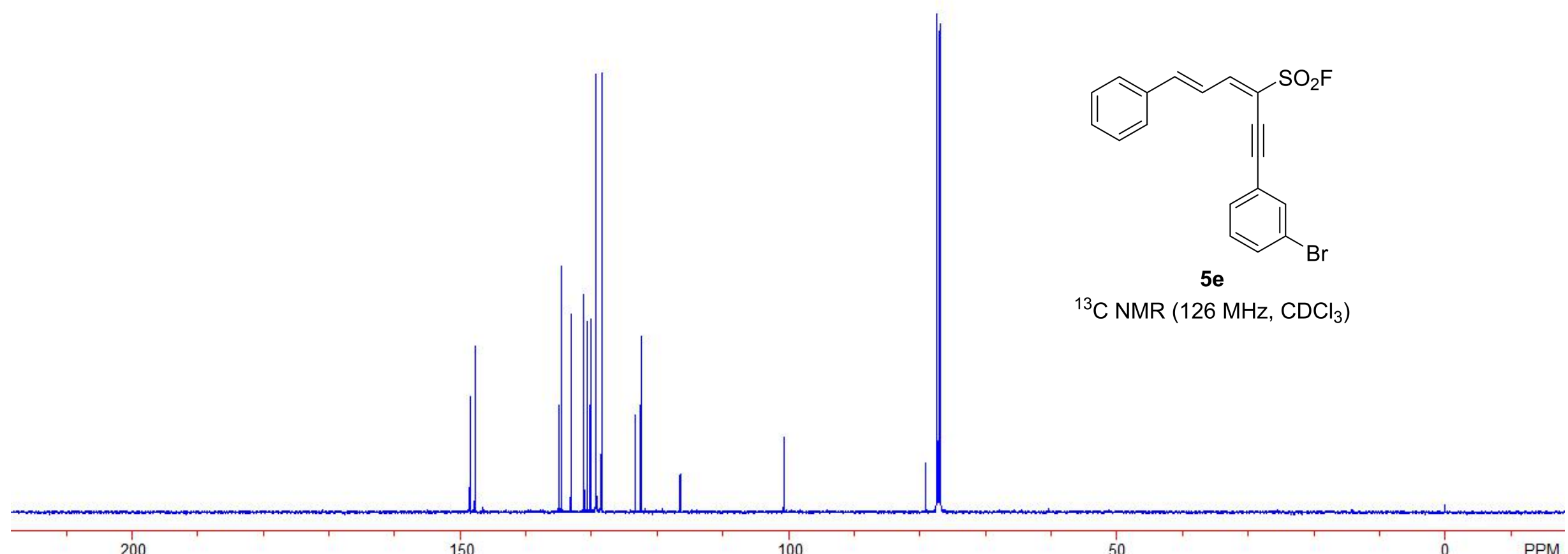




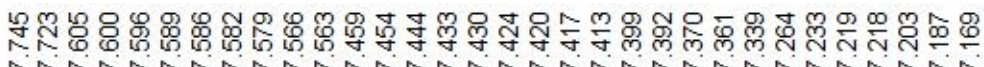

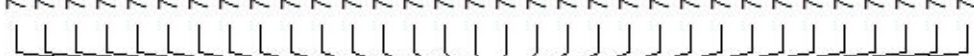

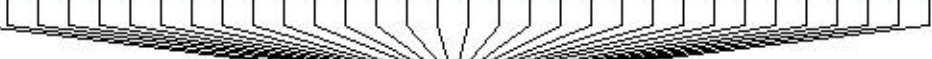

\section{Tा11iाi}

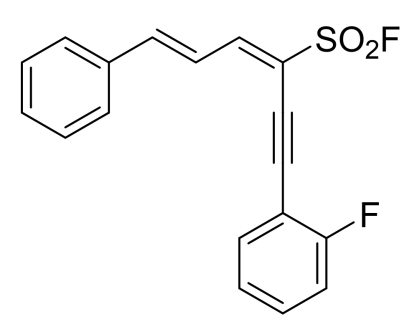

$5 f$

${ }^{1} \mathrm{H} \mathrm{NMR}\left(500 \mathrm{MHz}, \mathrm{CDCl}_{3}\right)$
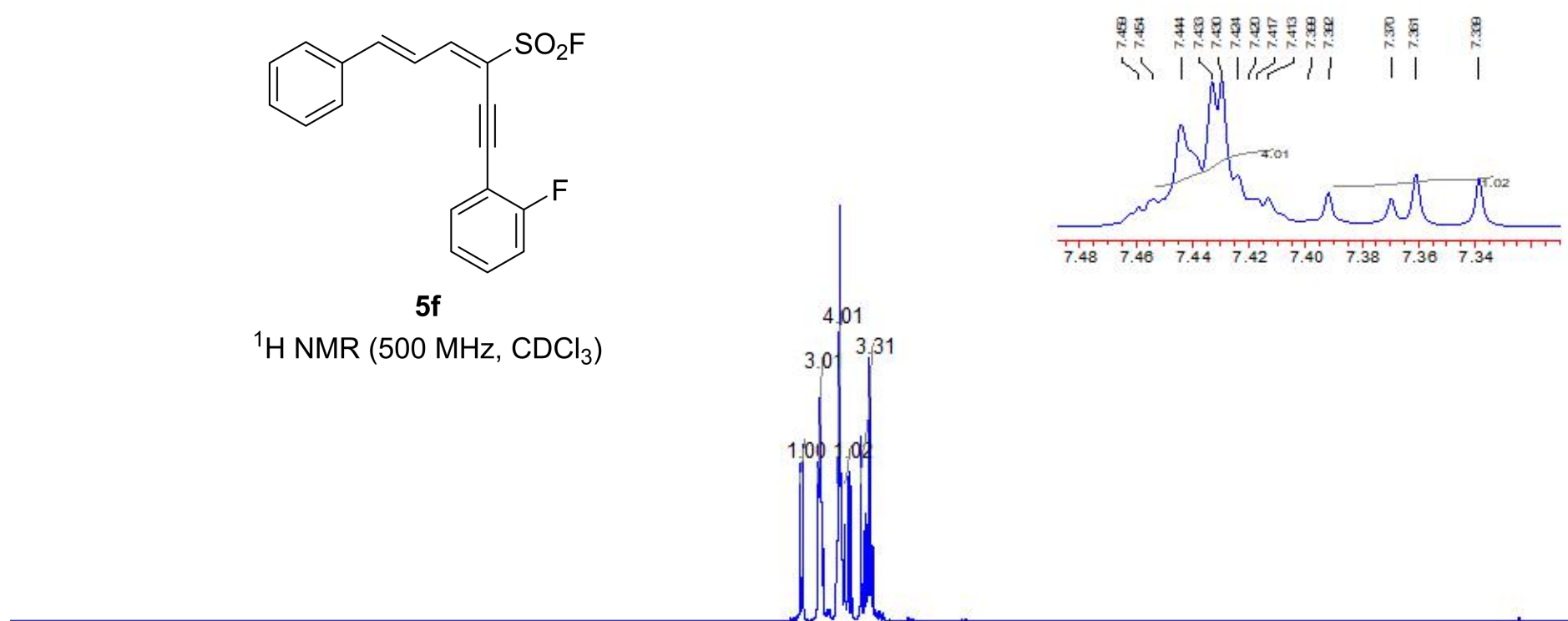

12

10

8

6

PPM 


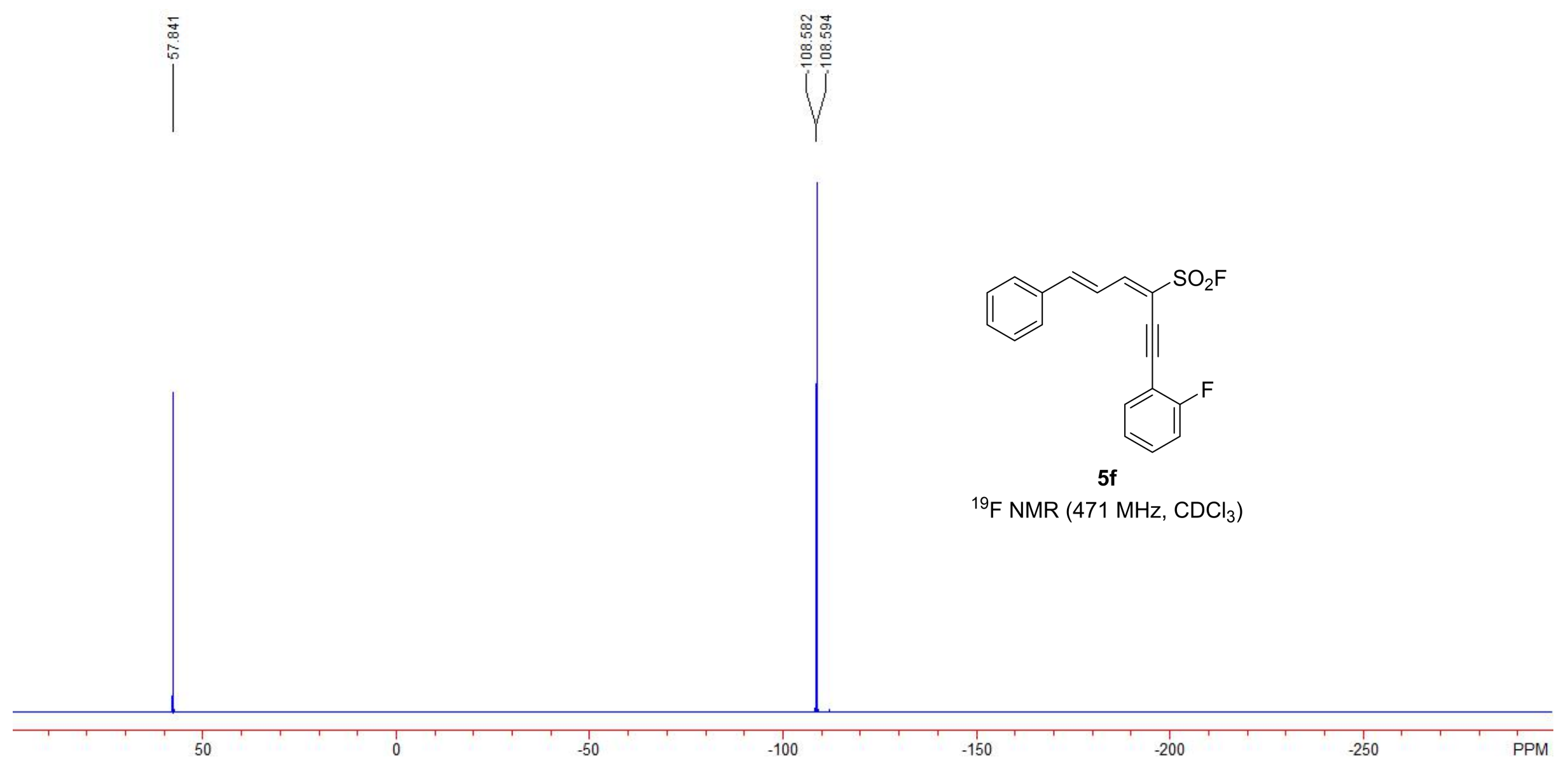



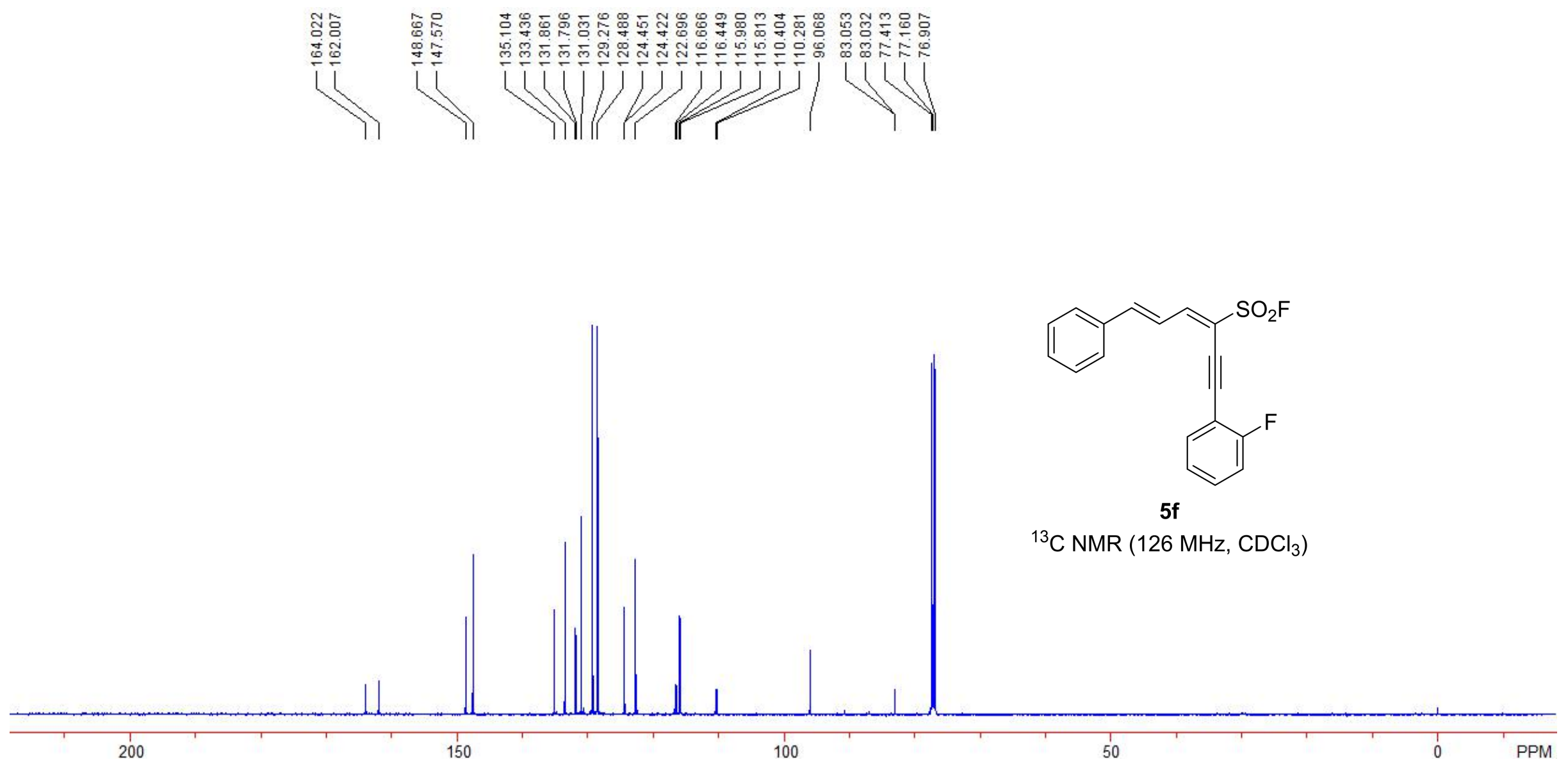


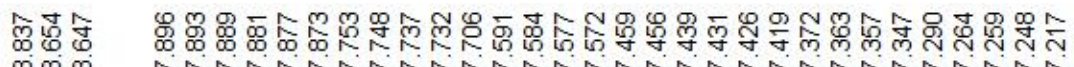

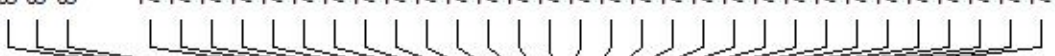 \\ 77 71 1 niा}

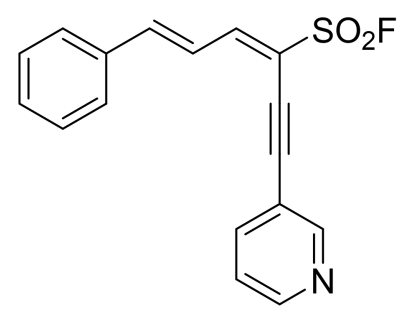

$5 g$

${ }^{1} \mathrm{H} \mathrm{NMR}\left(500 \mathrm{MHz}, \mathrm{CDCl}_{3}\right)$

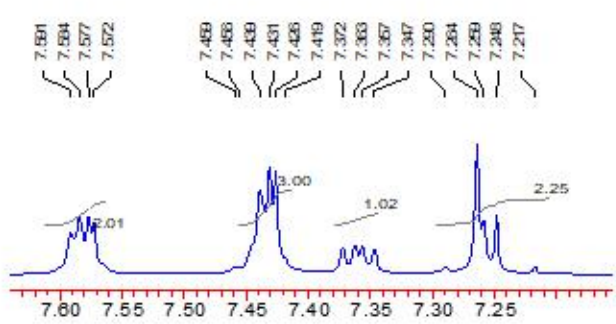

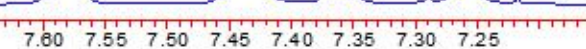

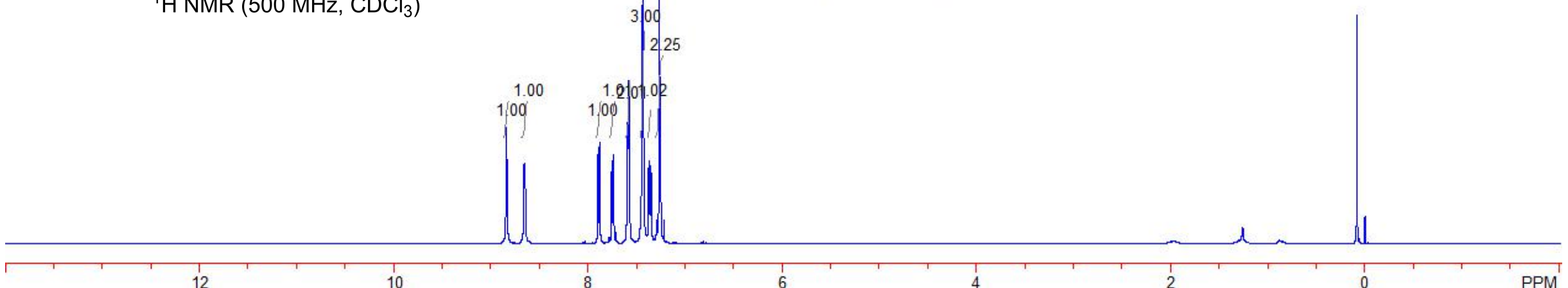




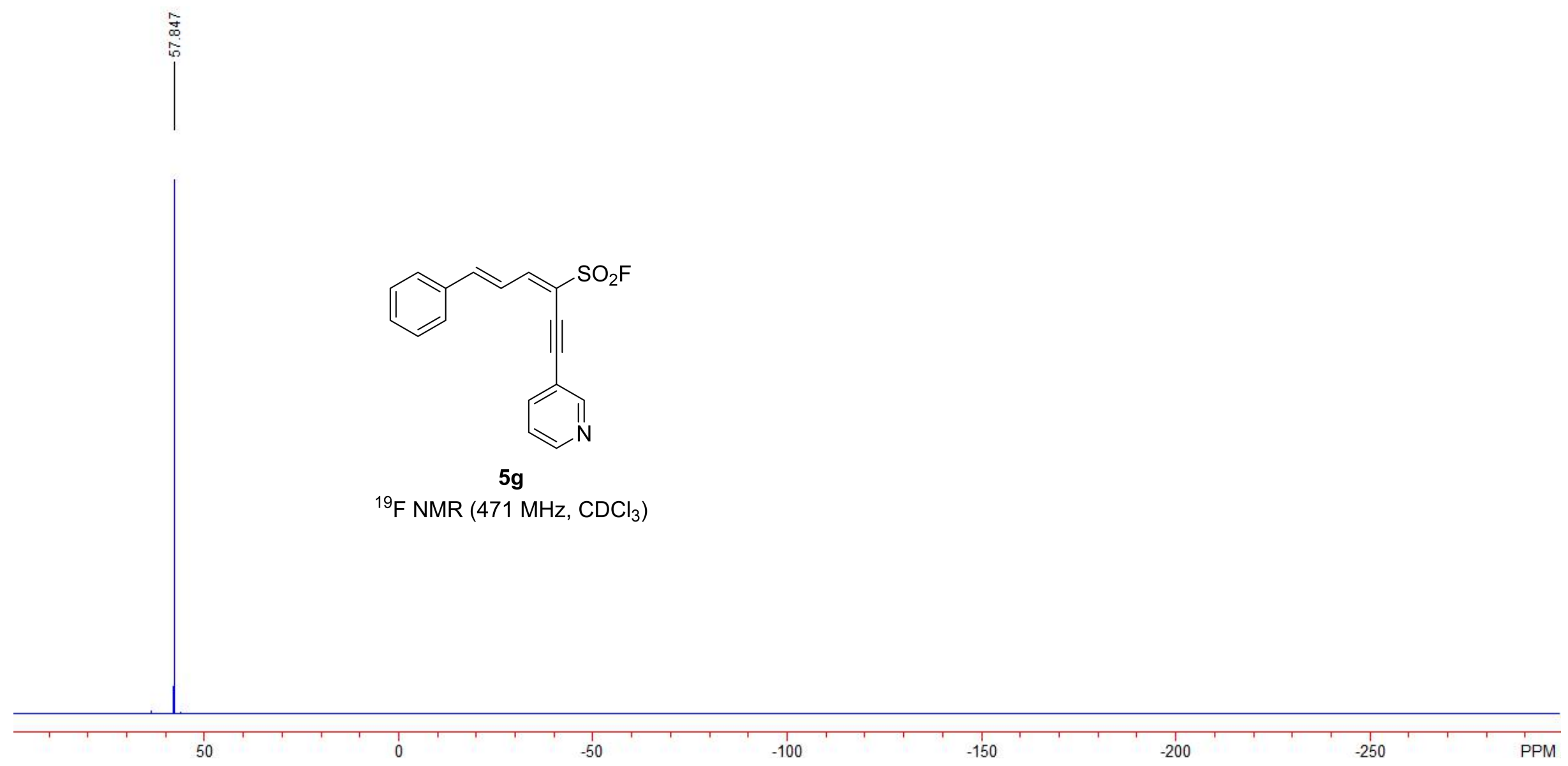




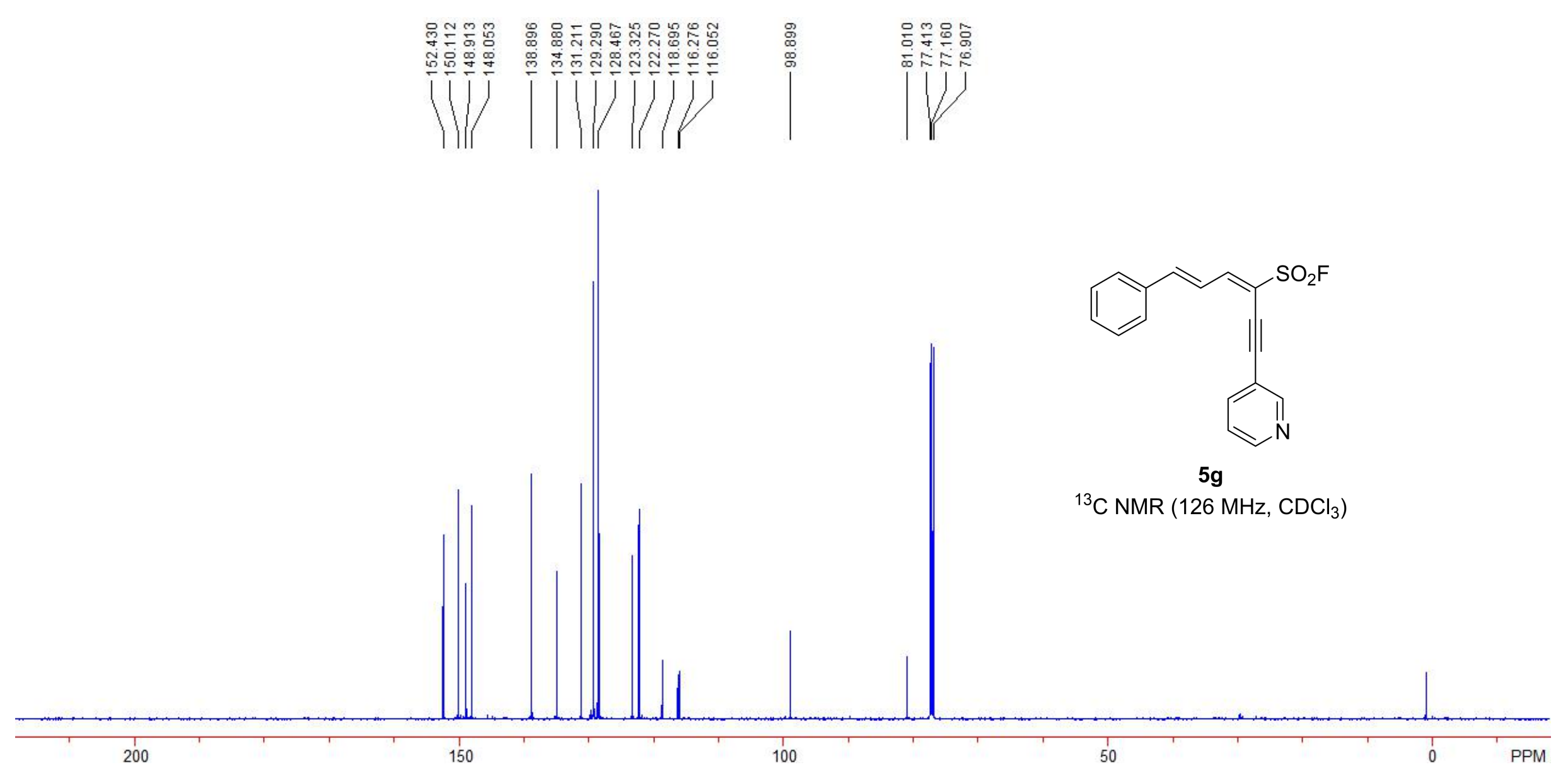




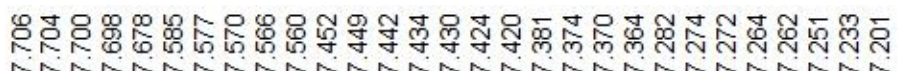

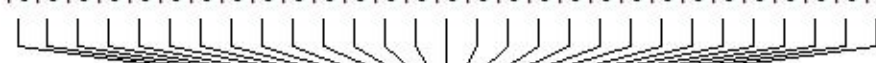

\section{TIII}

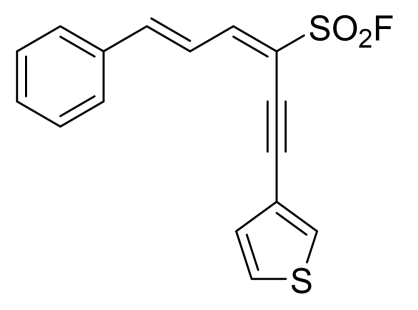

$5 \mathrm{~h}$

${ }^{1} \mathrm{H} \mathrm{NMR}\left(500 \mathrm{MHz}, \mathrm{CDCl}_{3}\right)$

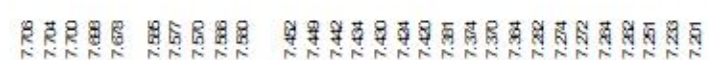

$\Leftrightarrow|m| \Leftrightarrow \mid$

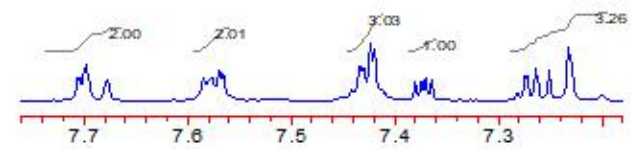

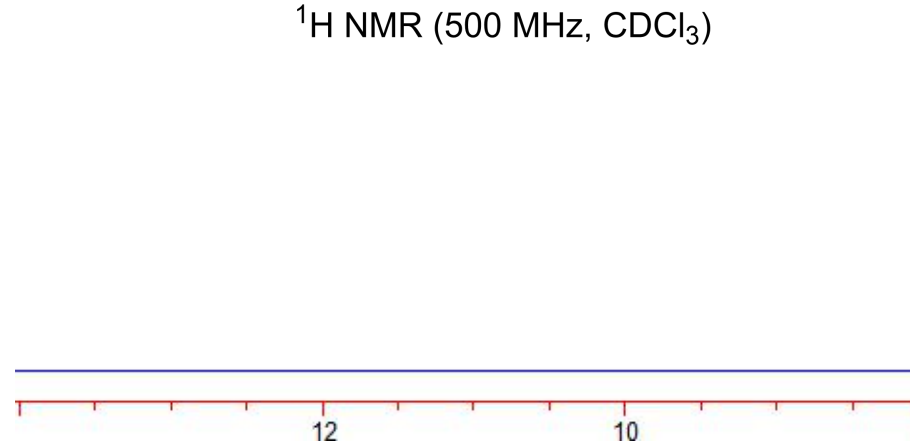




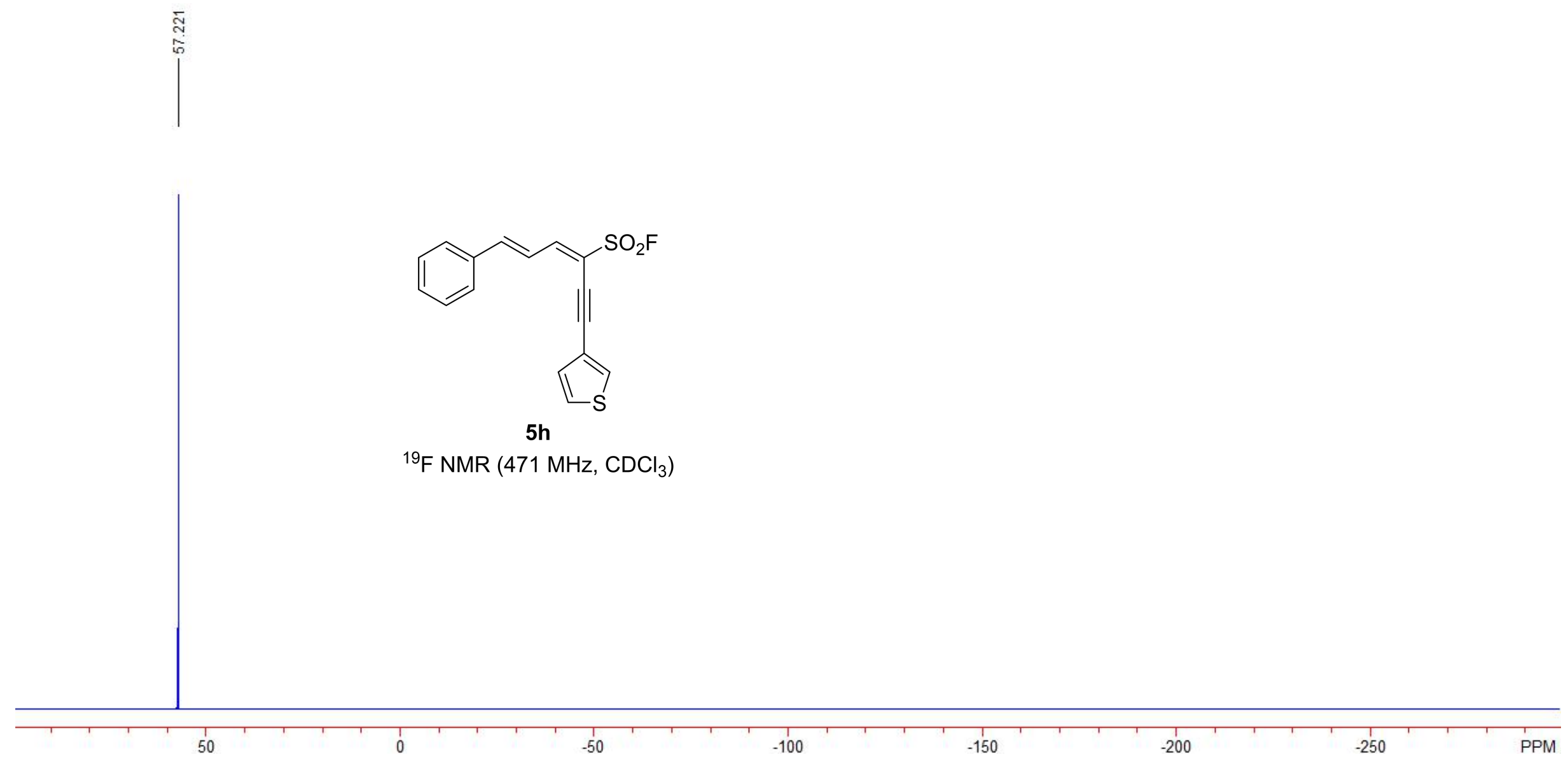



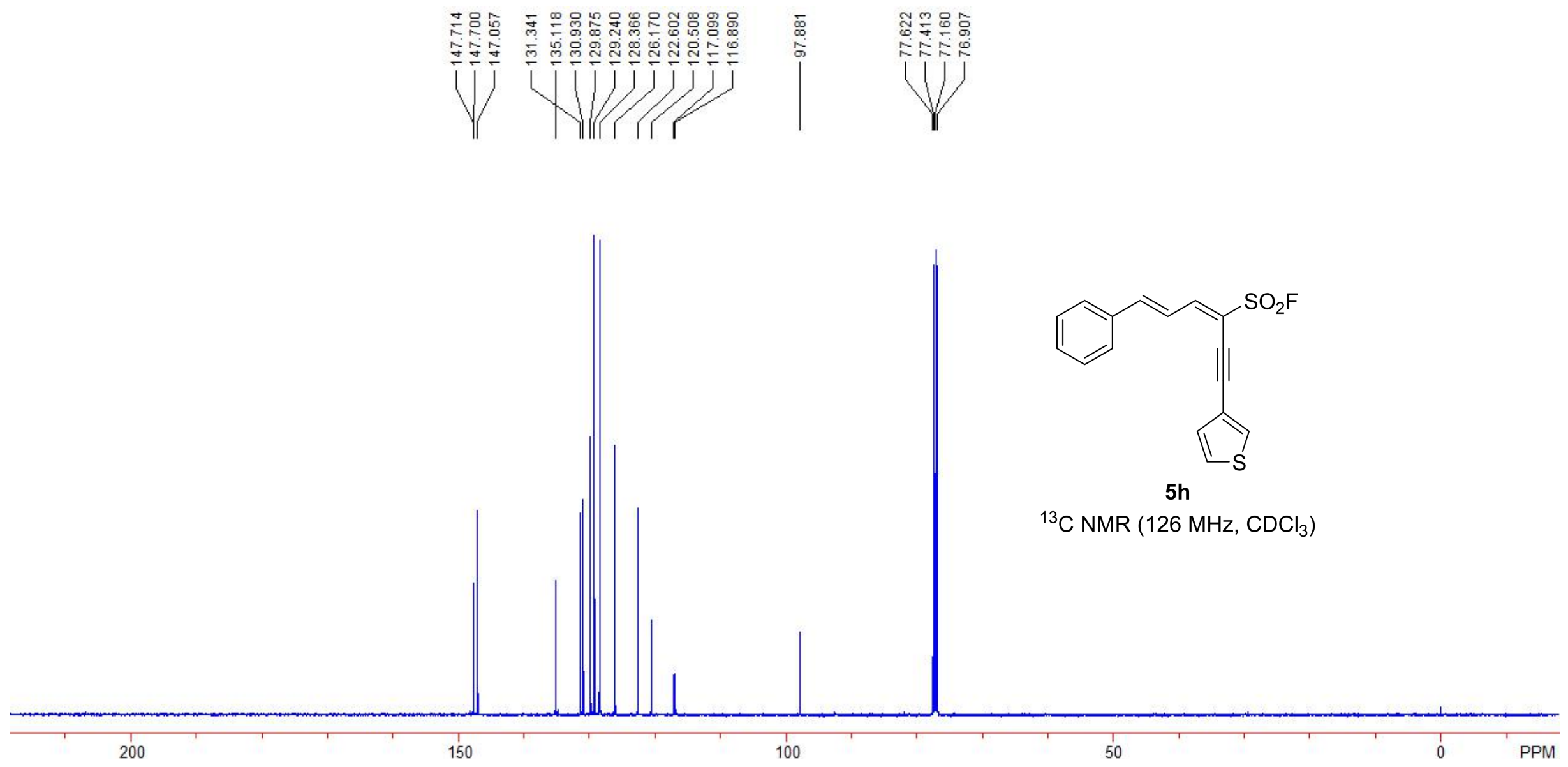


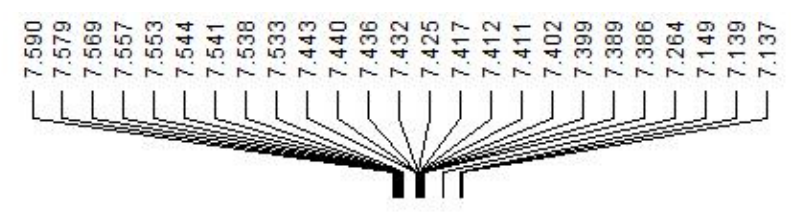

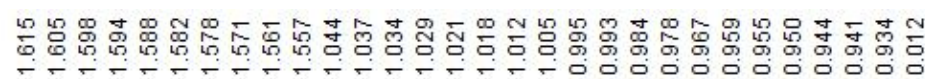

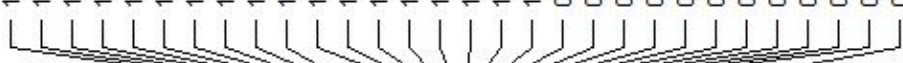
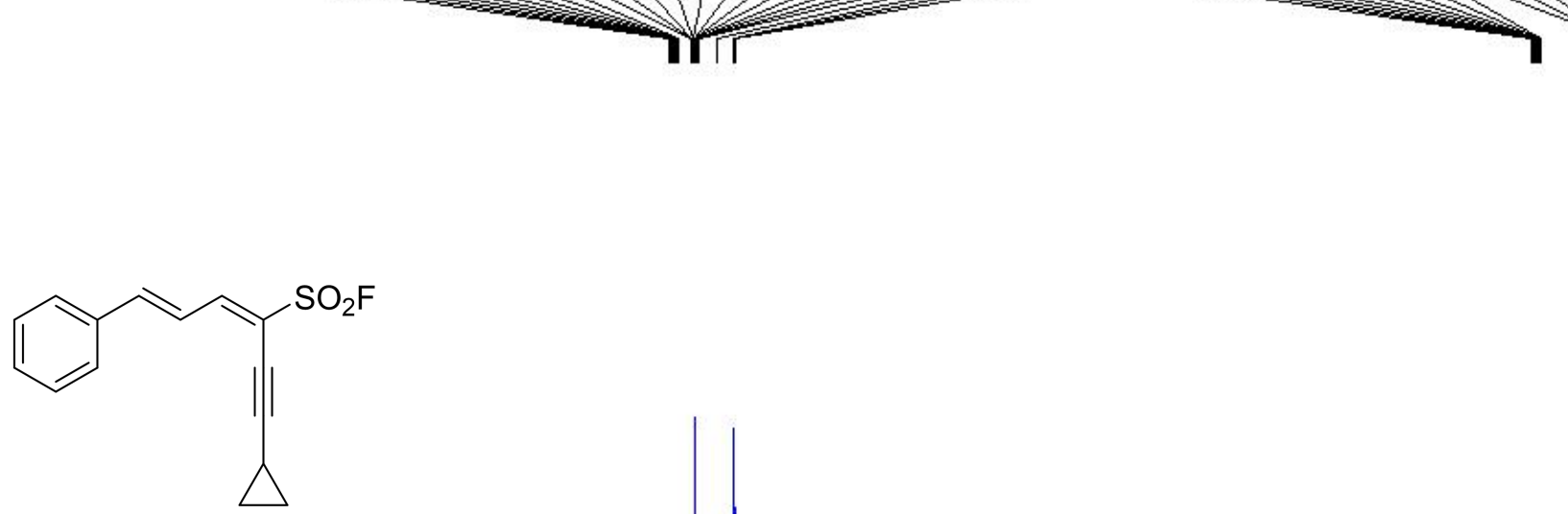

$5 i$

${ }^{1} \mathrm{H}$ NMR $\left(500 \mathrm{MHz}, \mathrm{CDCl}_{3}\right)$
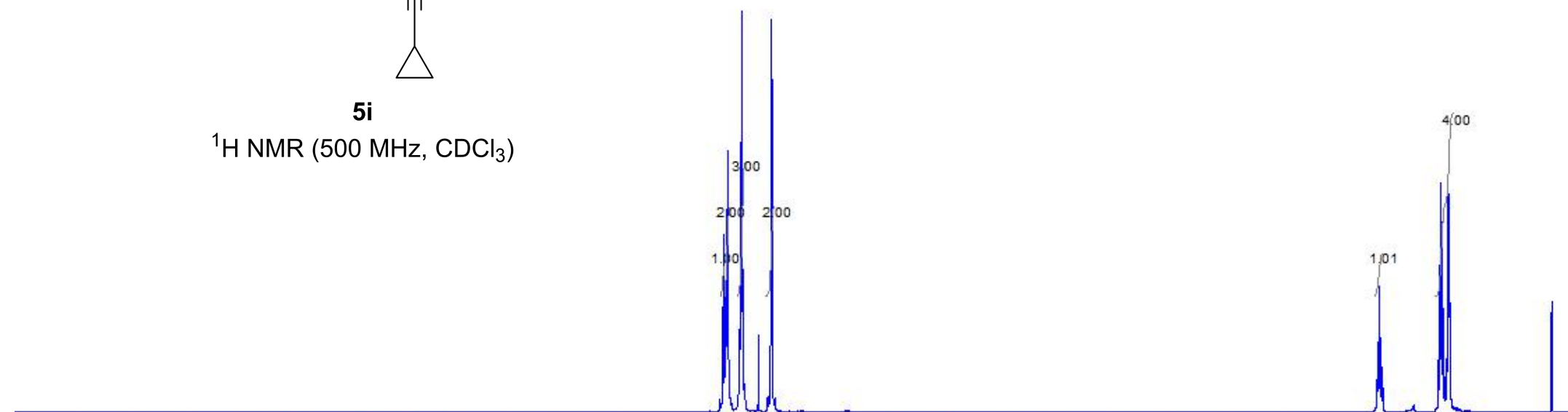

12

10

8

6

4

2

0

PPM 


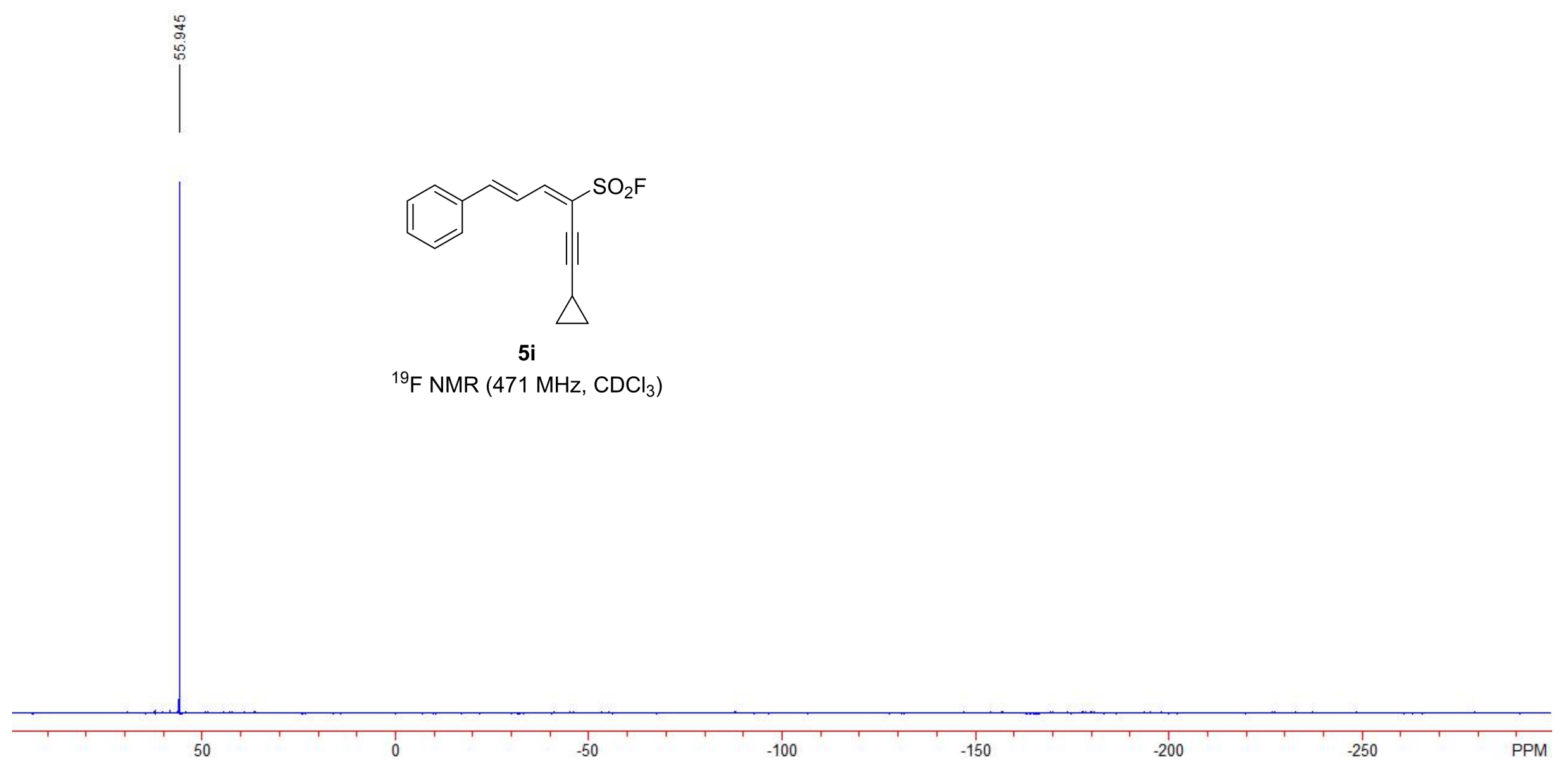




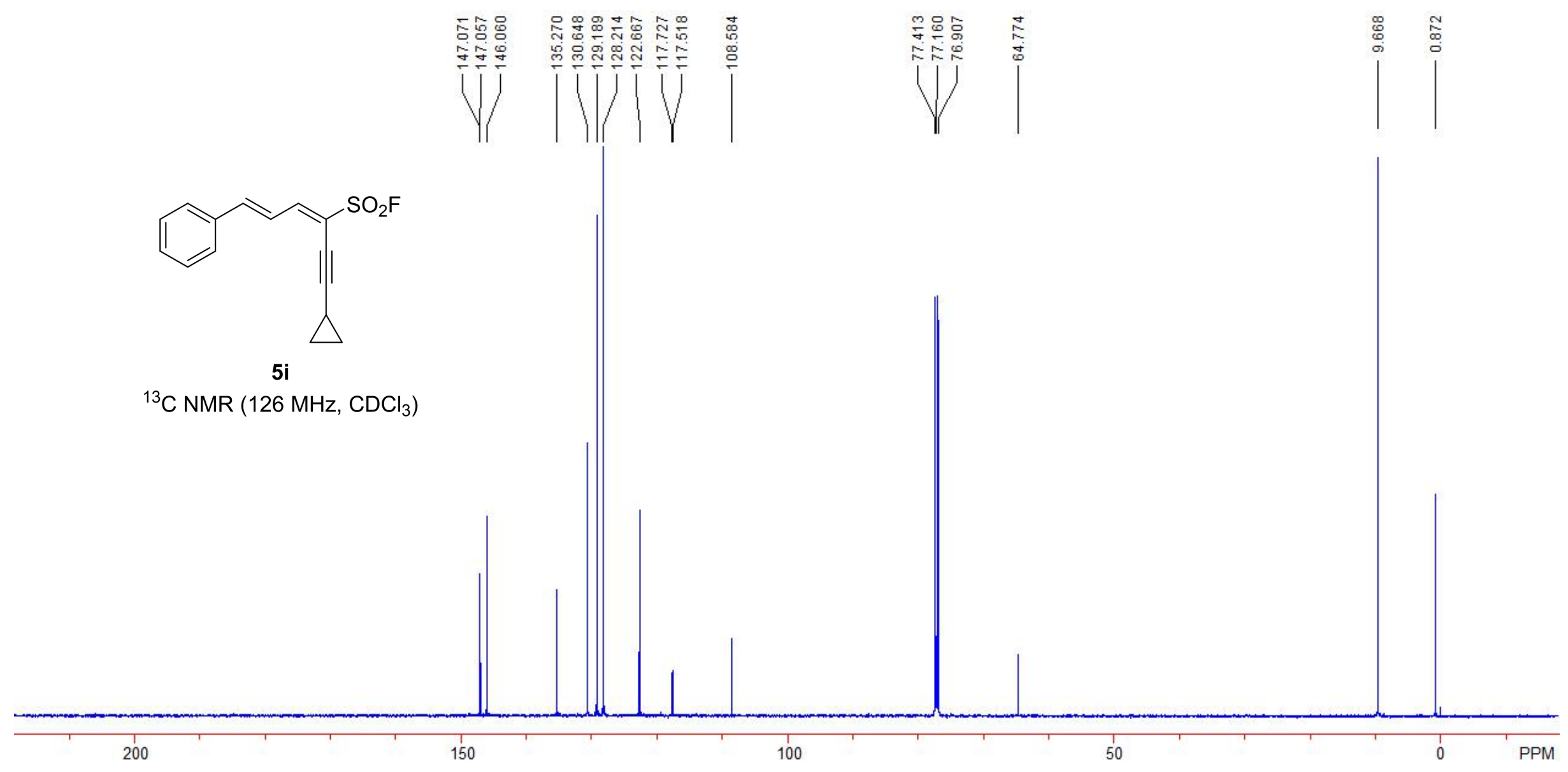



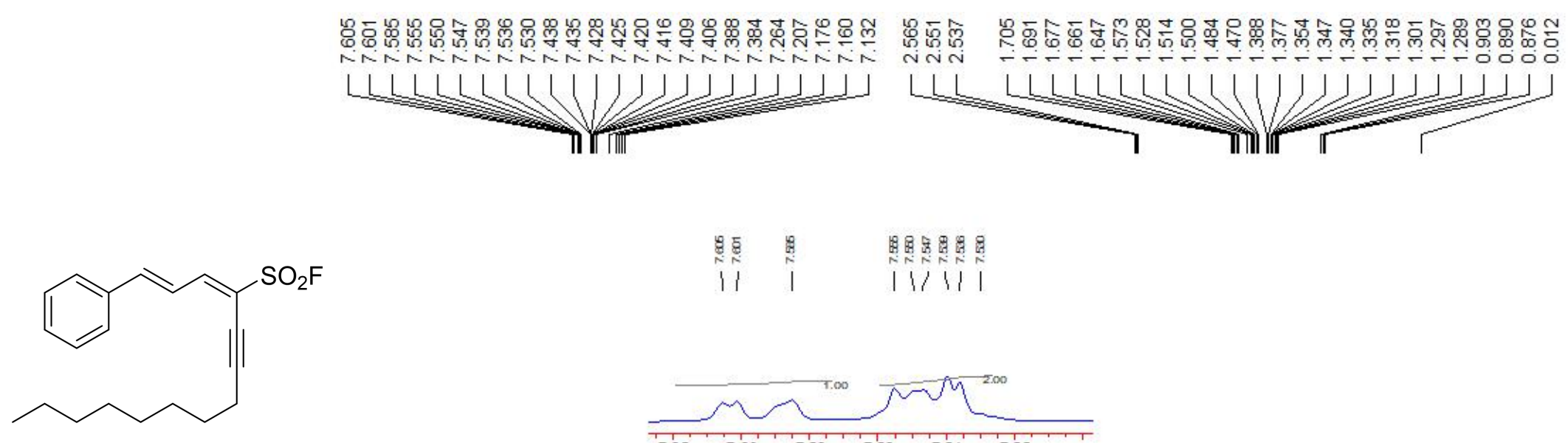

5j

${ }^{1} \mathrm{H}$ NMR $\left(500 \mathrm{MHz}, \mathrm{CDCl}_{3}\right)$
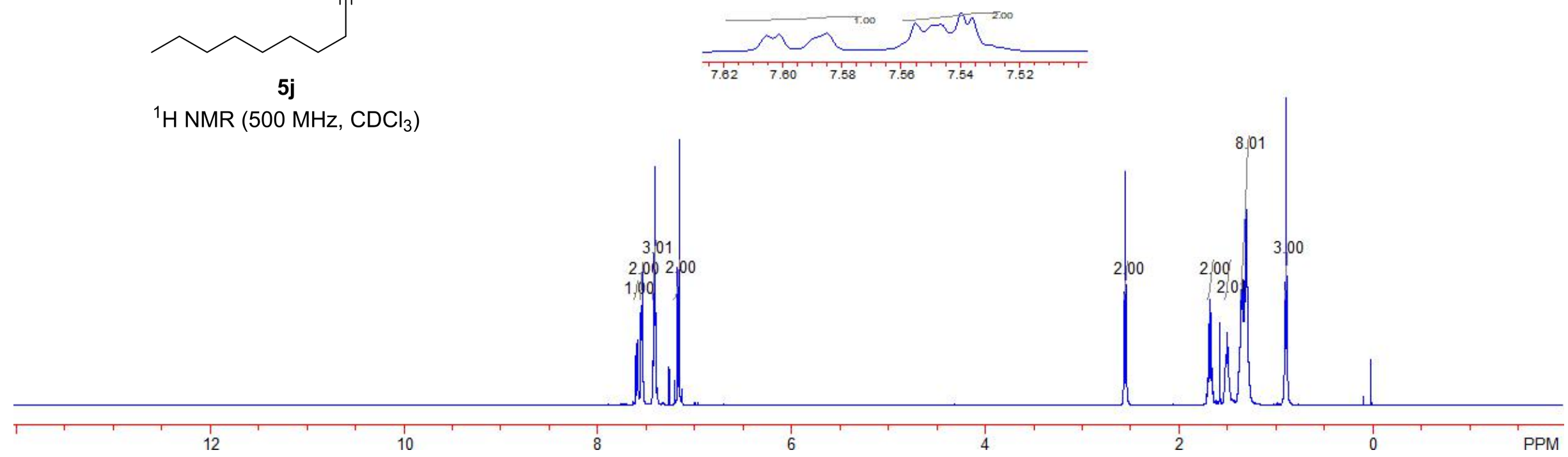

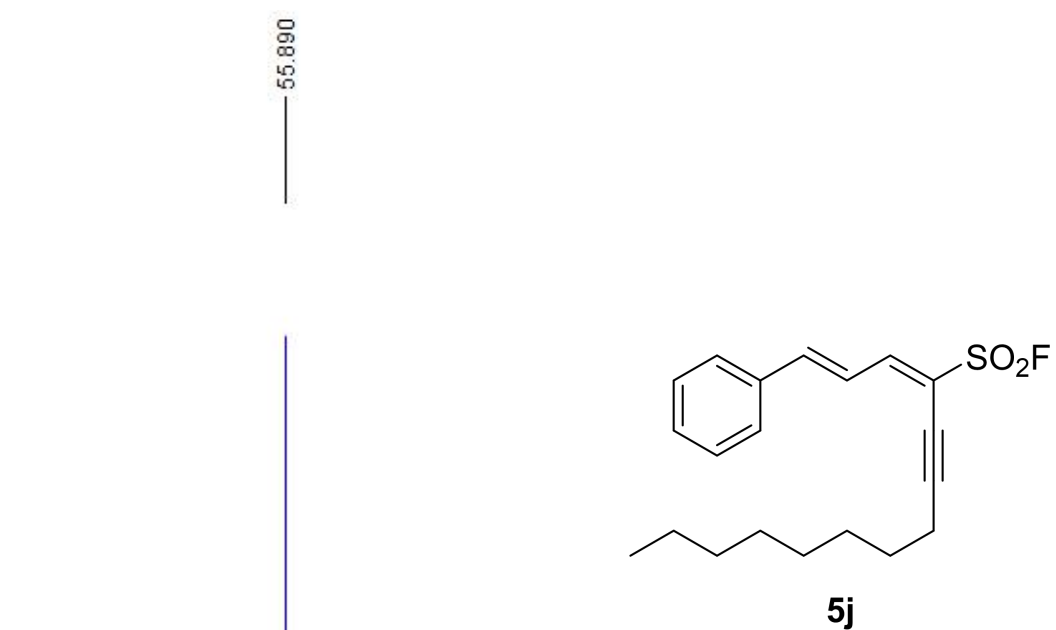

${ }^{19} \mathrm{~F} \mathrm{NMR}\left(471 \mathrm{MHz}, \mathrm{CDCl}_{3}\right)$ 


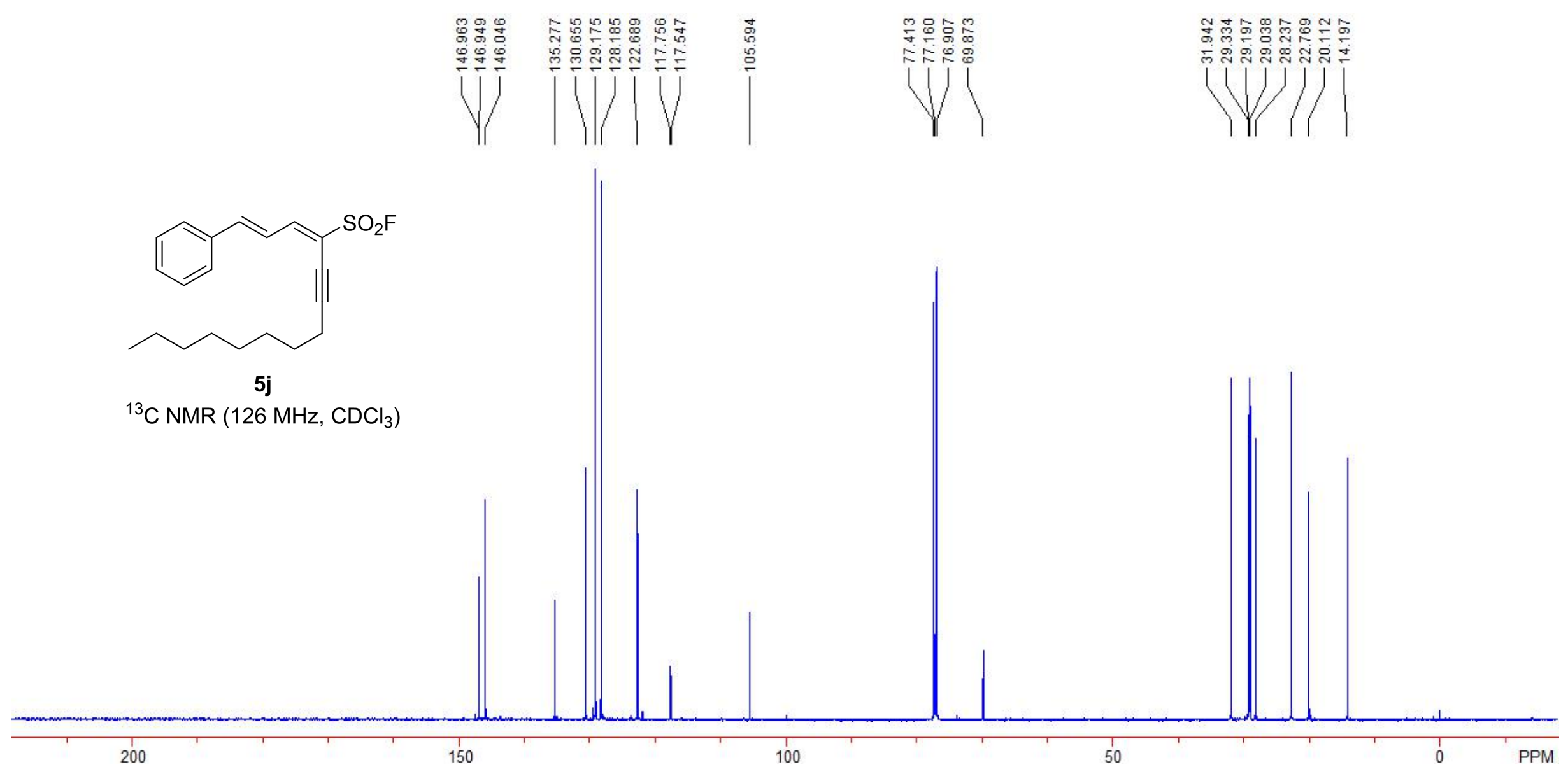




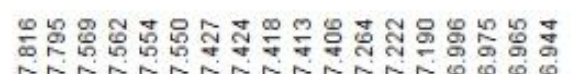

UMUUUJJUJ

과 1110

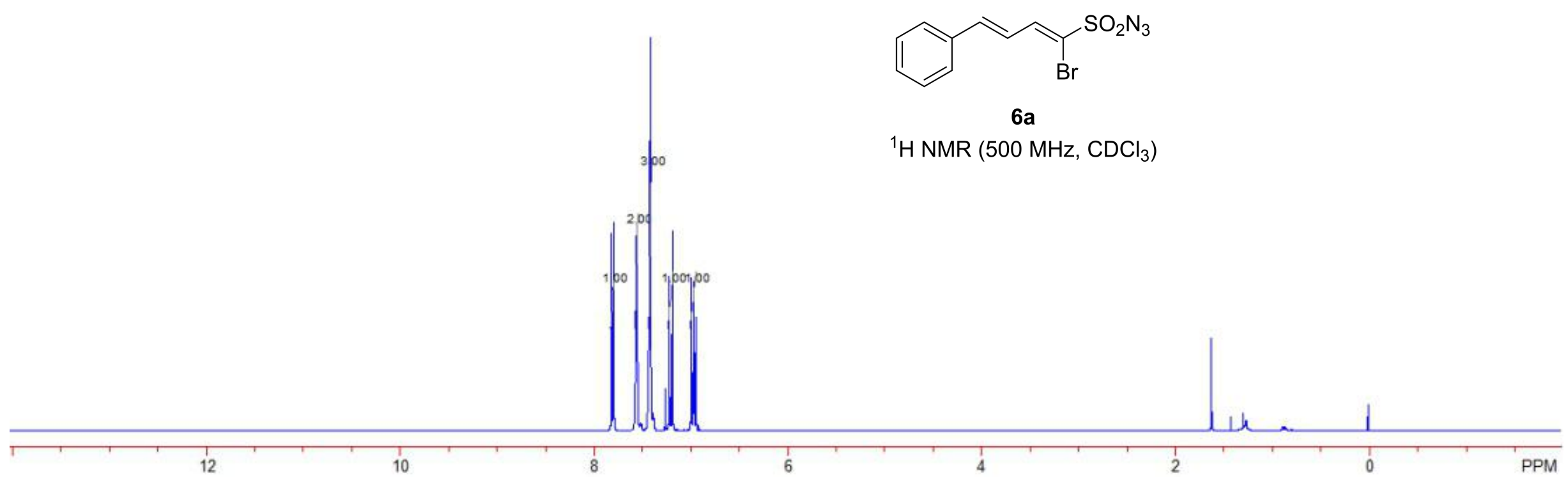



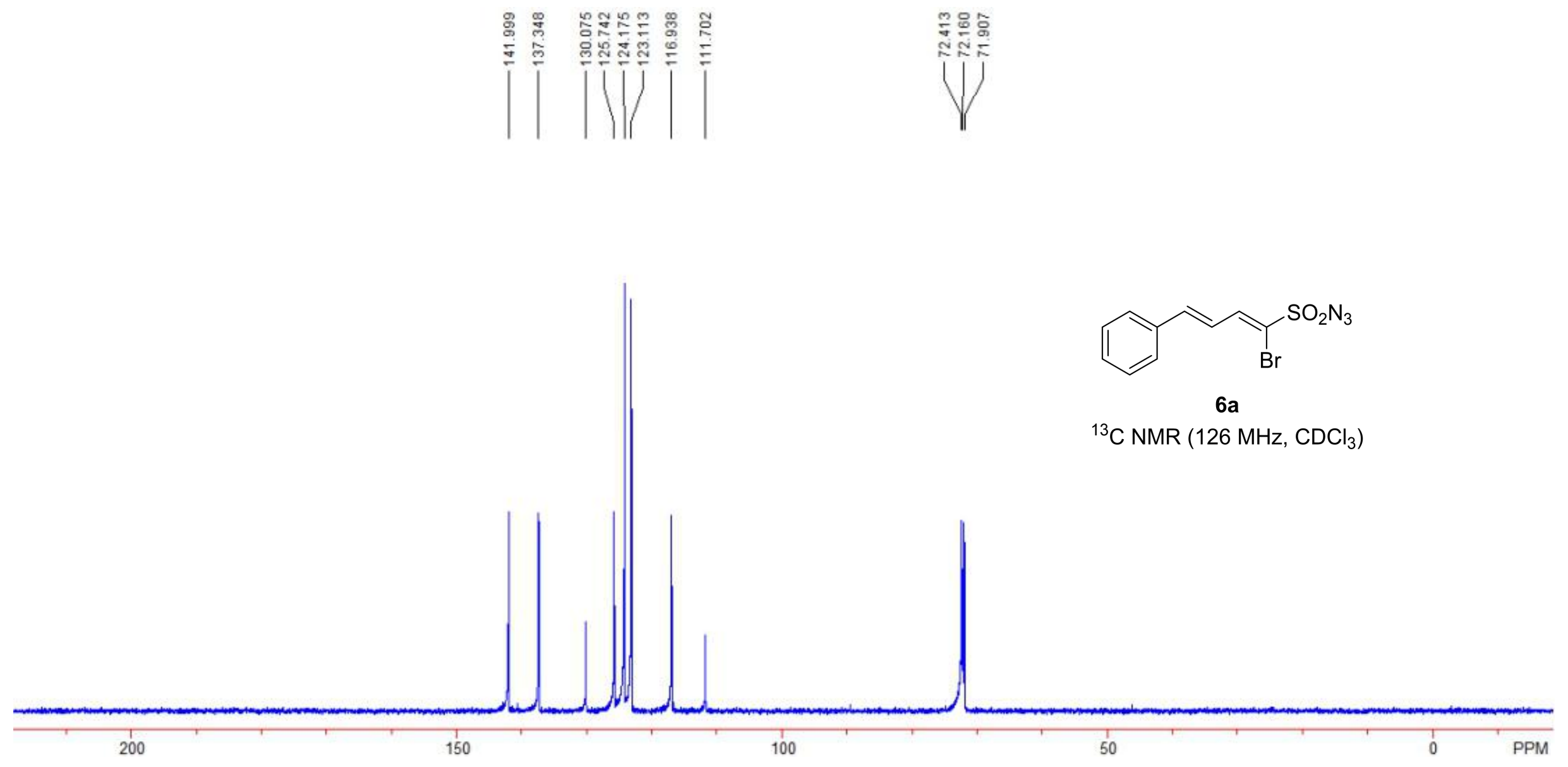

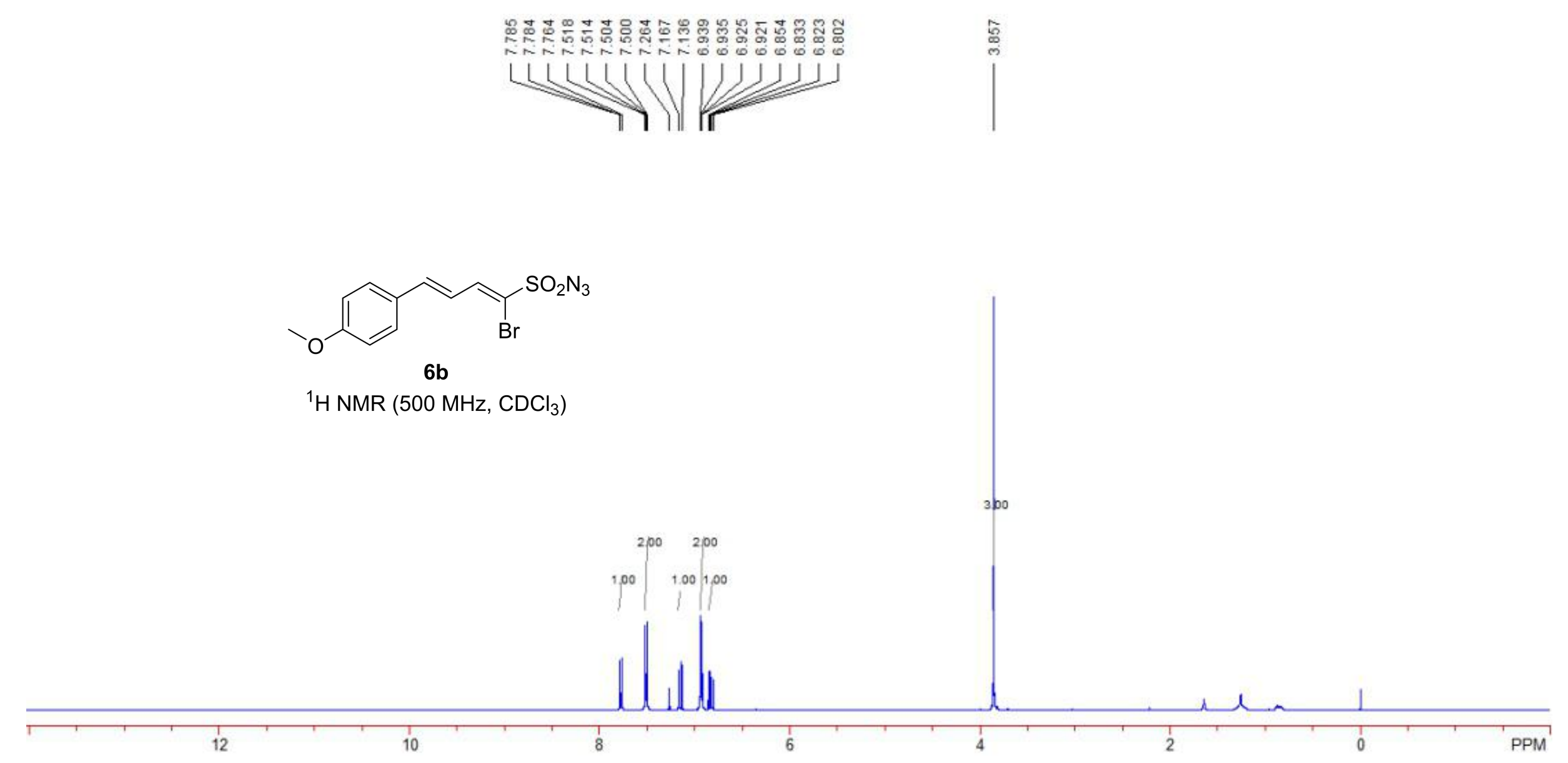


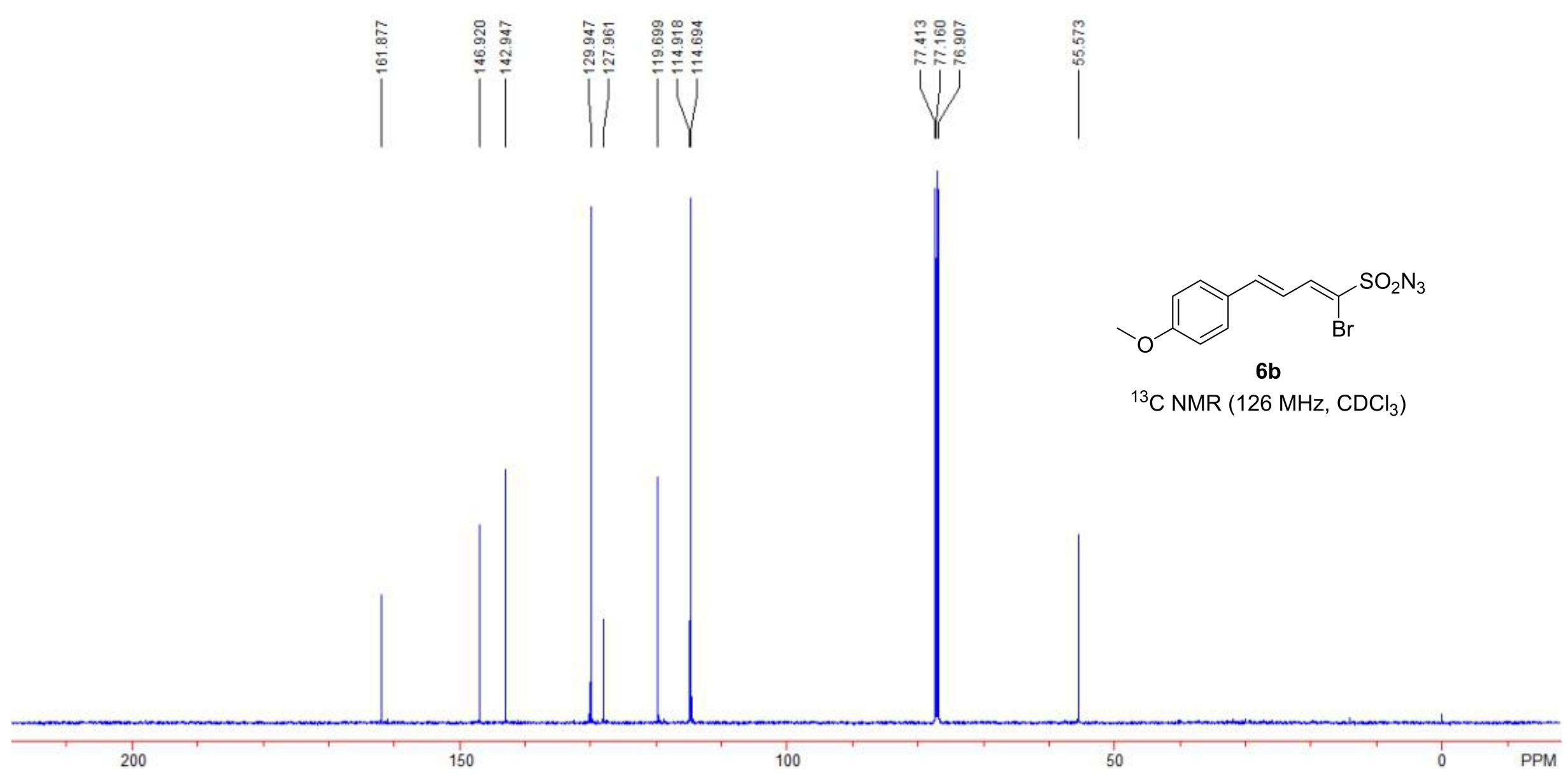




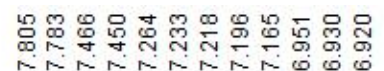

$[1(1) 1 j j)$

I N

品

$\mid$

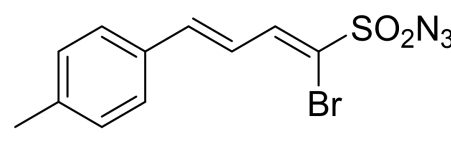

6c

${ }^{1} \mathrm{H}$ NMR (500 MHz, $\mathrm{CDCl}_{3}$ )

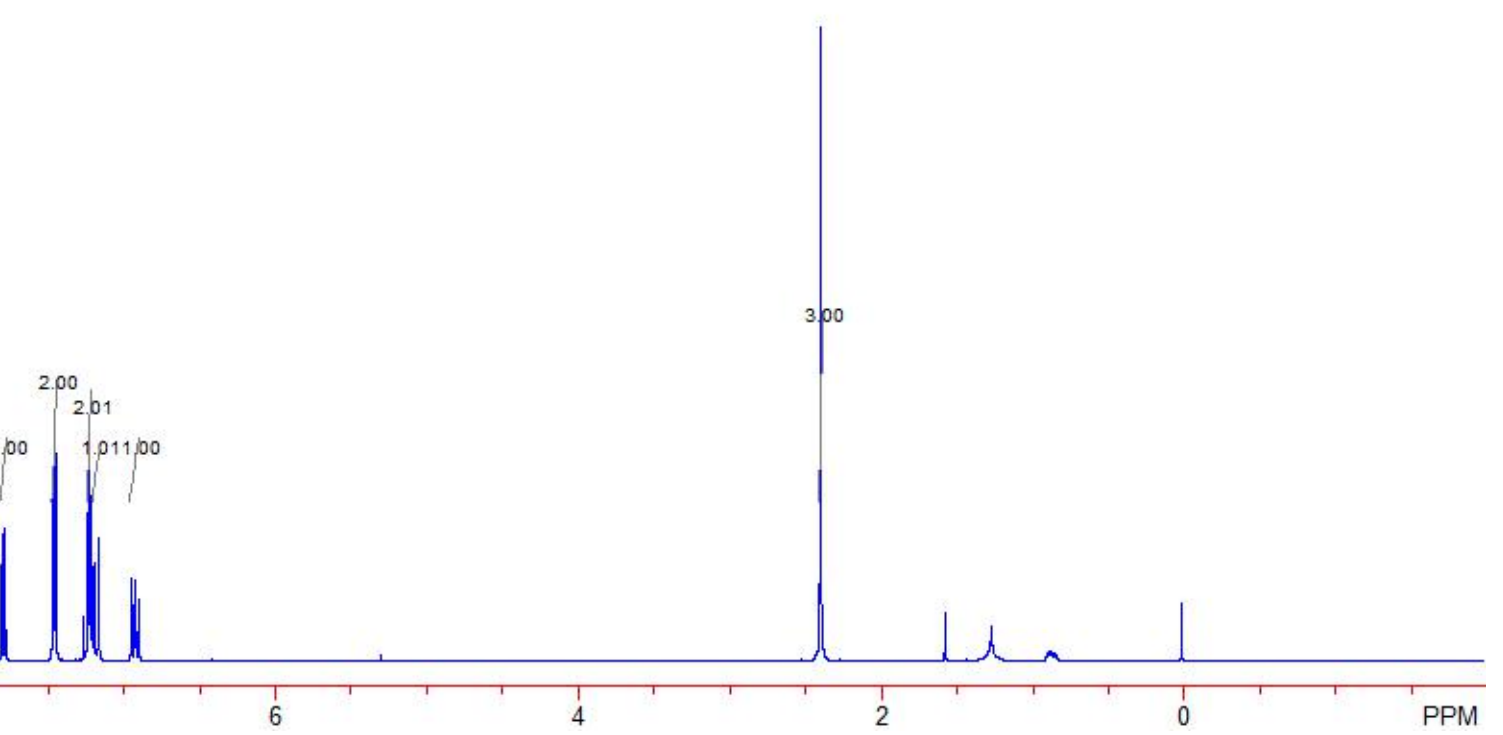



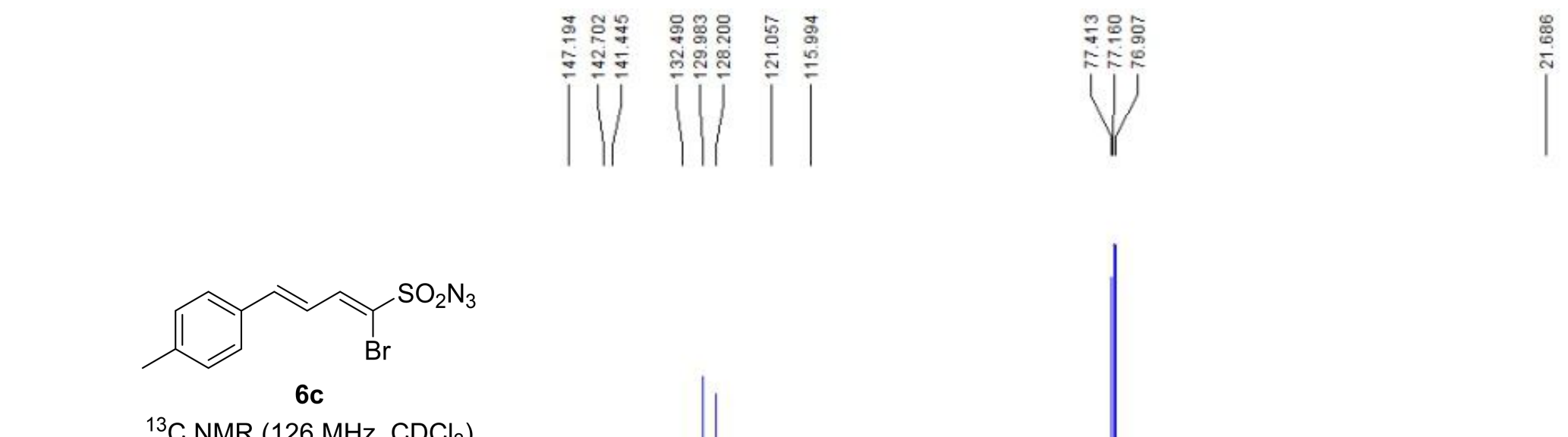

${ }^{13} \mathrm{C}$ NMR $\left(126 \mathrm{MHz}, \mathrm{CDCl}_{3}\right)$

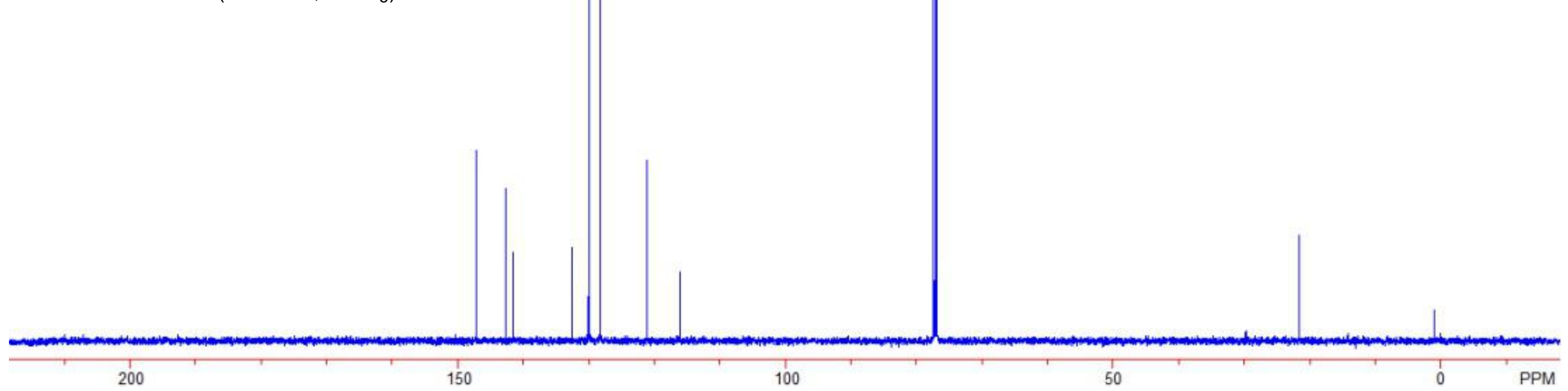




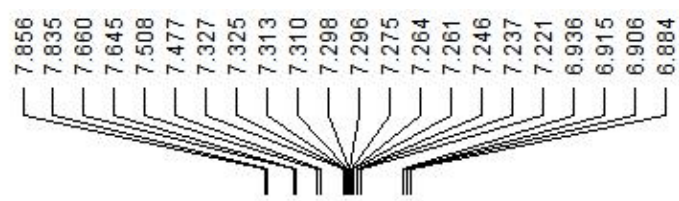

$\stackrel{\sqrt[3]{3}}{\pi}$

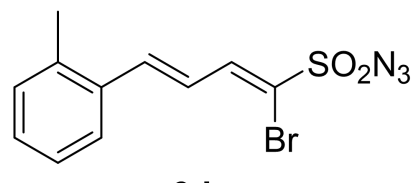

6d

${ }^{1} \mathrm{H}$ NMR $\left(500 \mathrm{MHz}, \mathrm{CDCl}_{3}\right)$

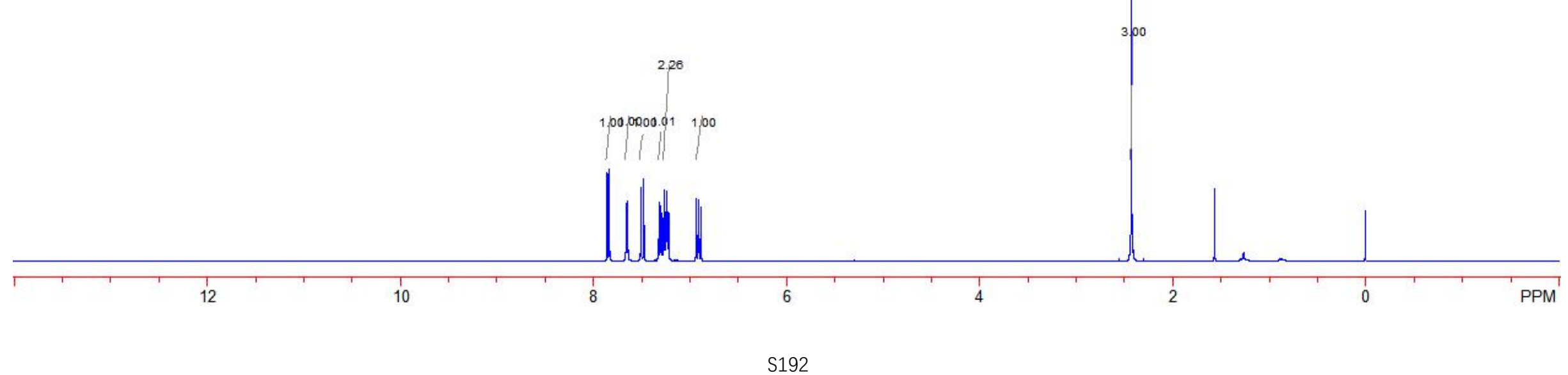



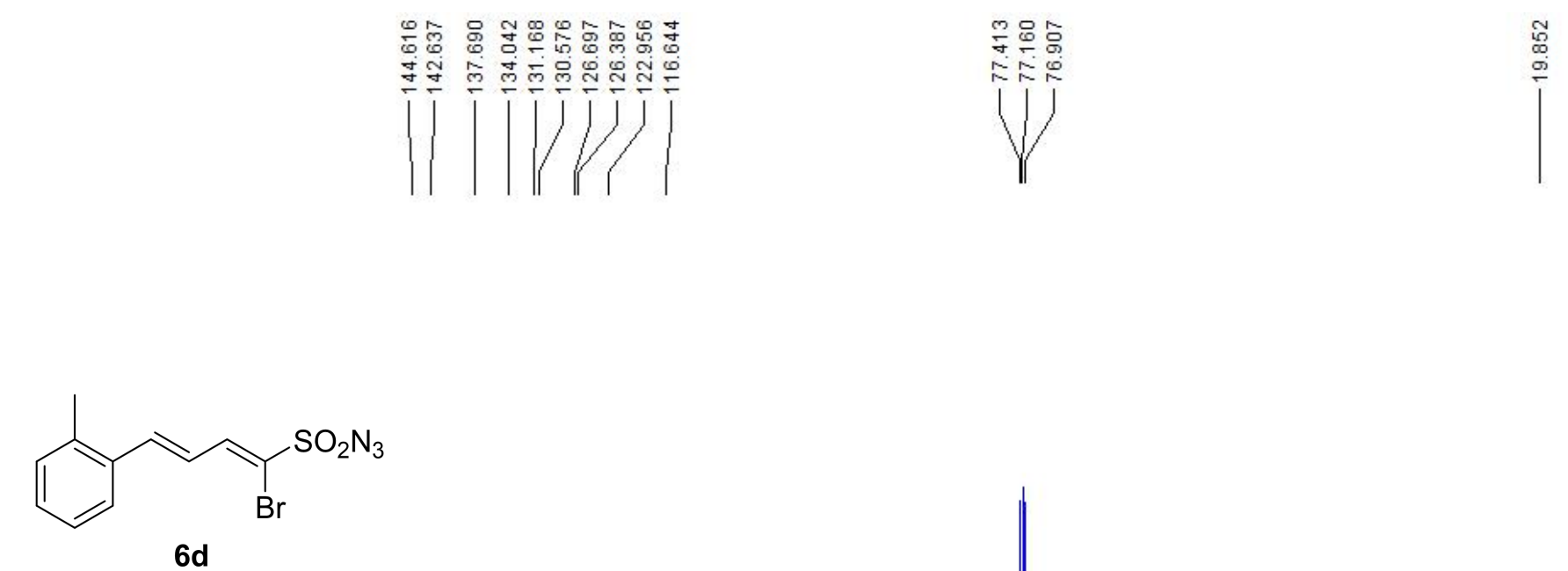

${ }^{13} \mathrm{C}$ NMR (126 MHz, $\mathrm{CDCl}_{3}$ )

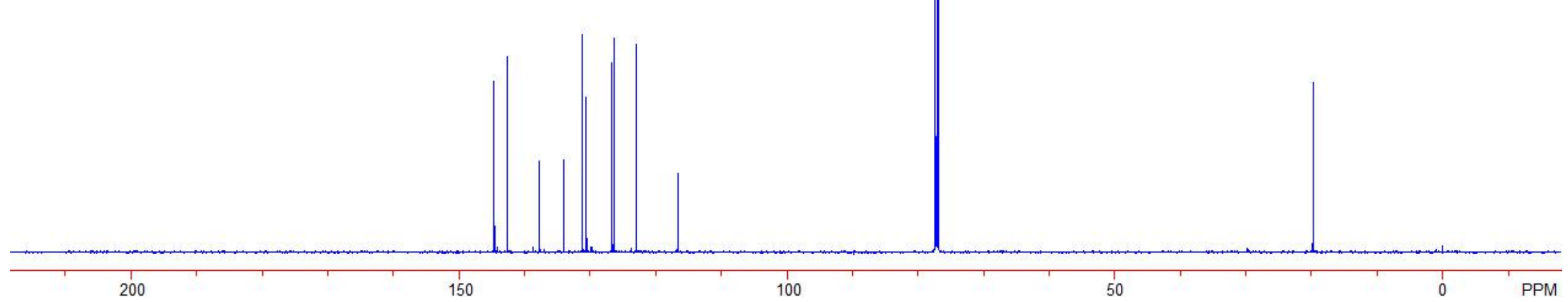




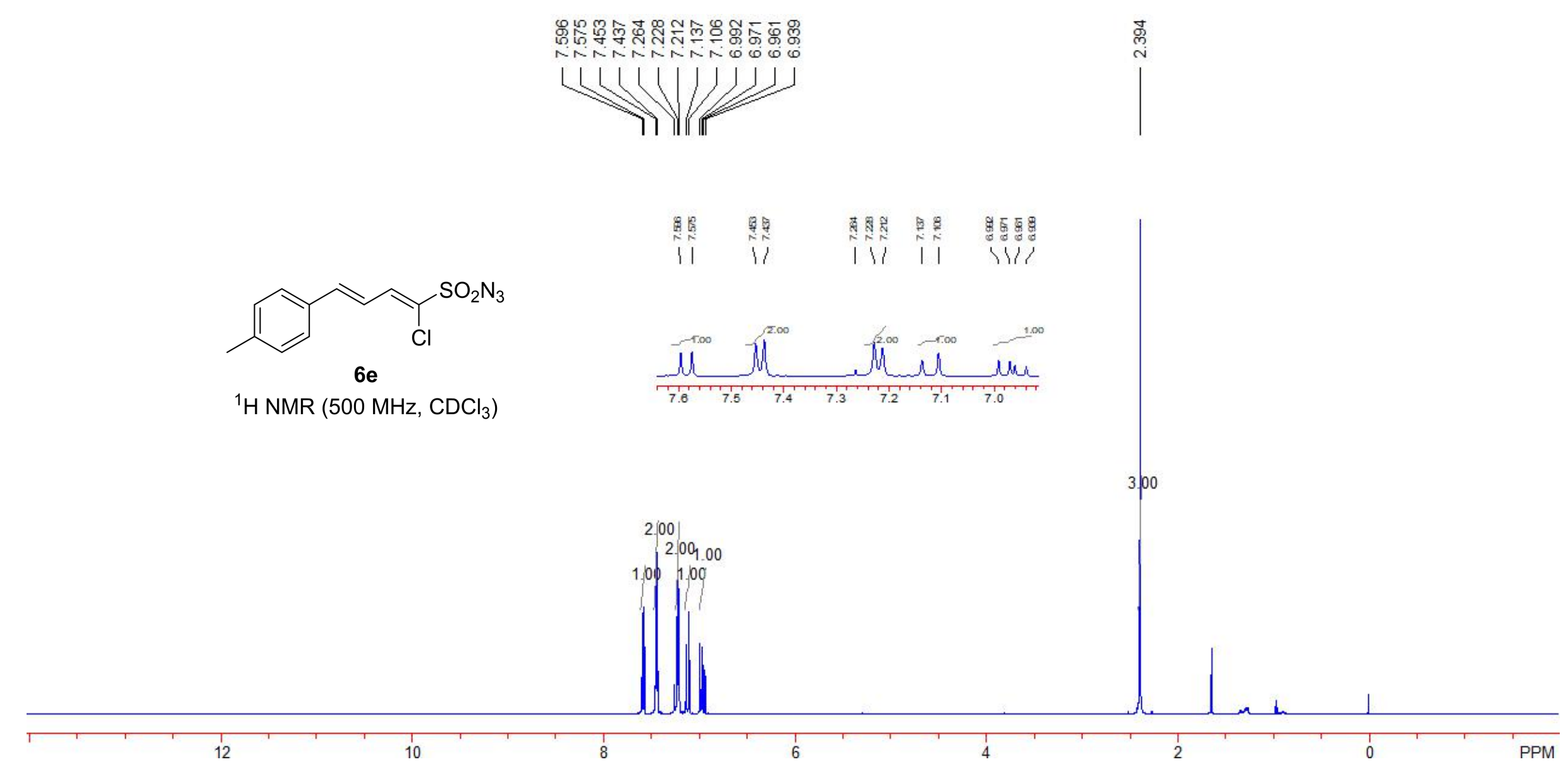



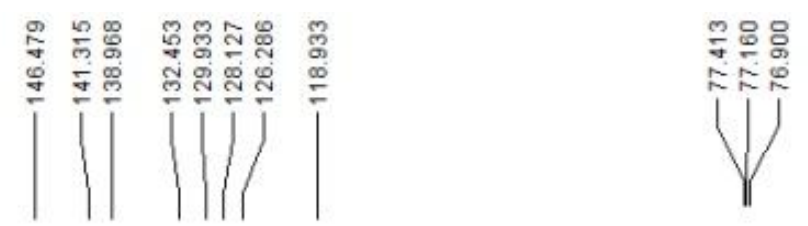

号

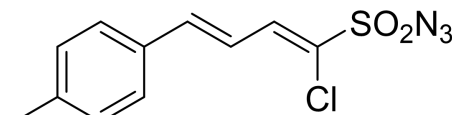

$6 e$

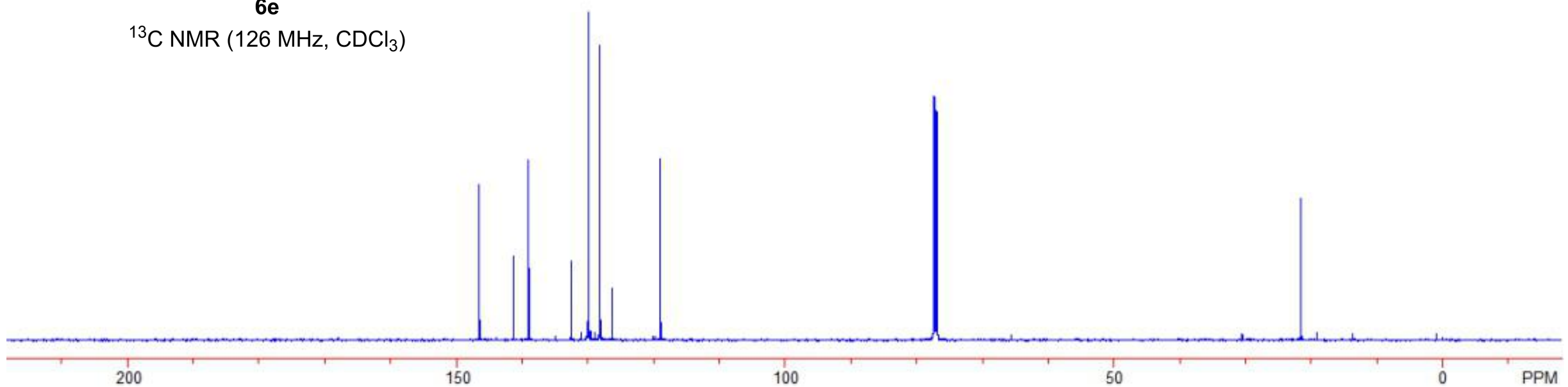




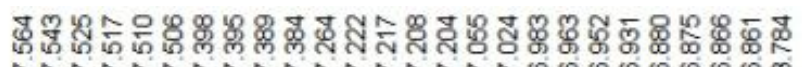

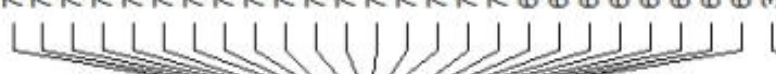
ㄱา 1 IIIT
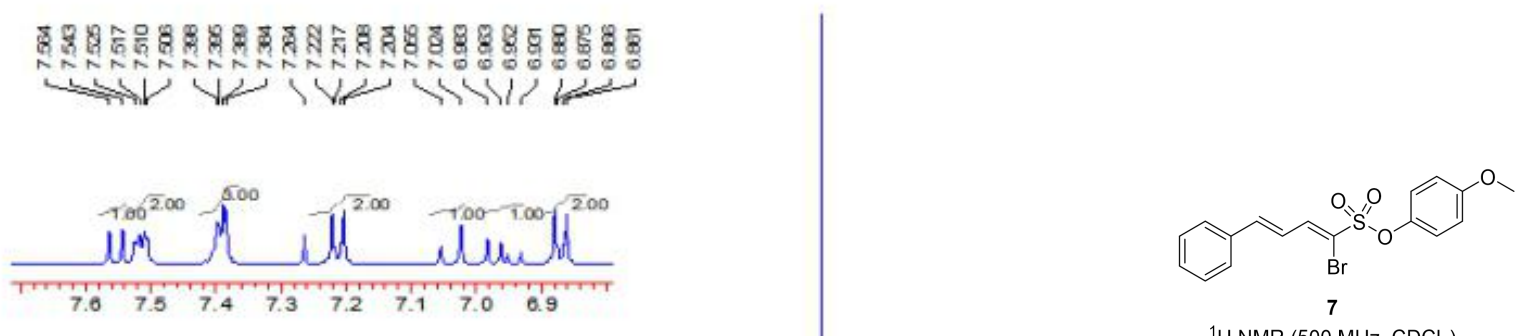

${ }^{1} \mathrm{H} \mathrm{NMR}\left(500 \mathrm{MHz}, \mathrm{CDCl}_{3}\right)$

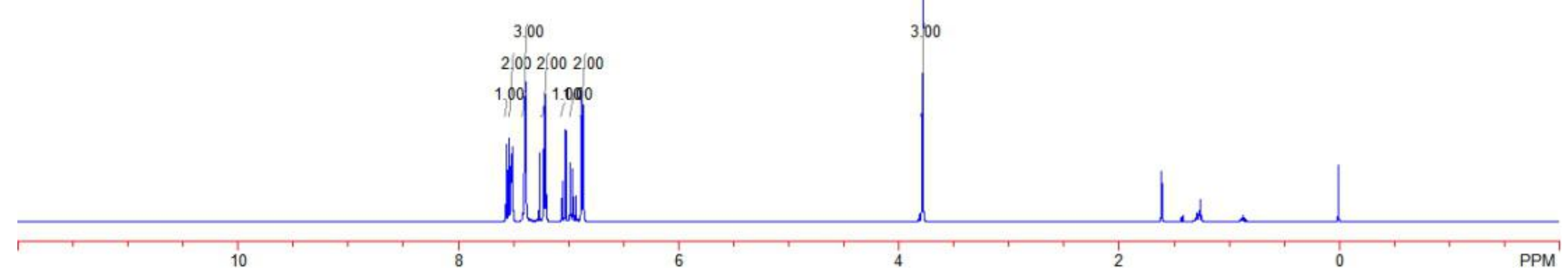



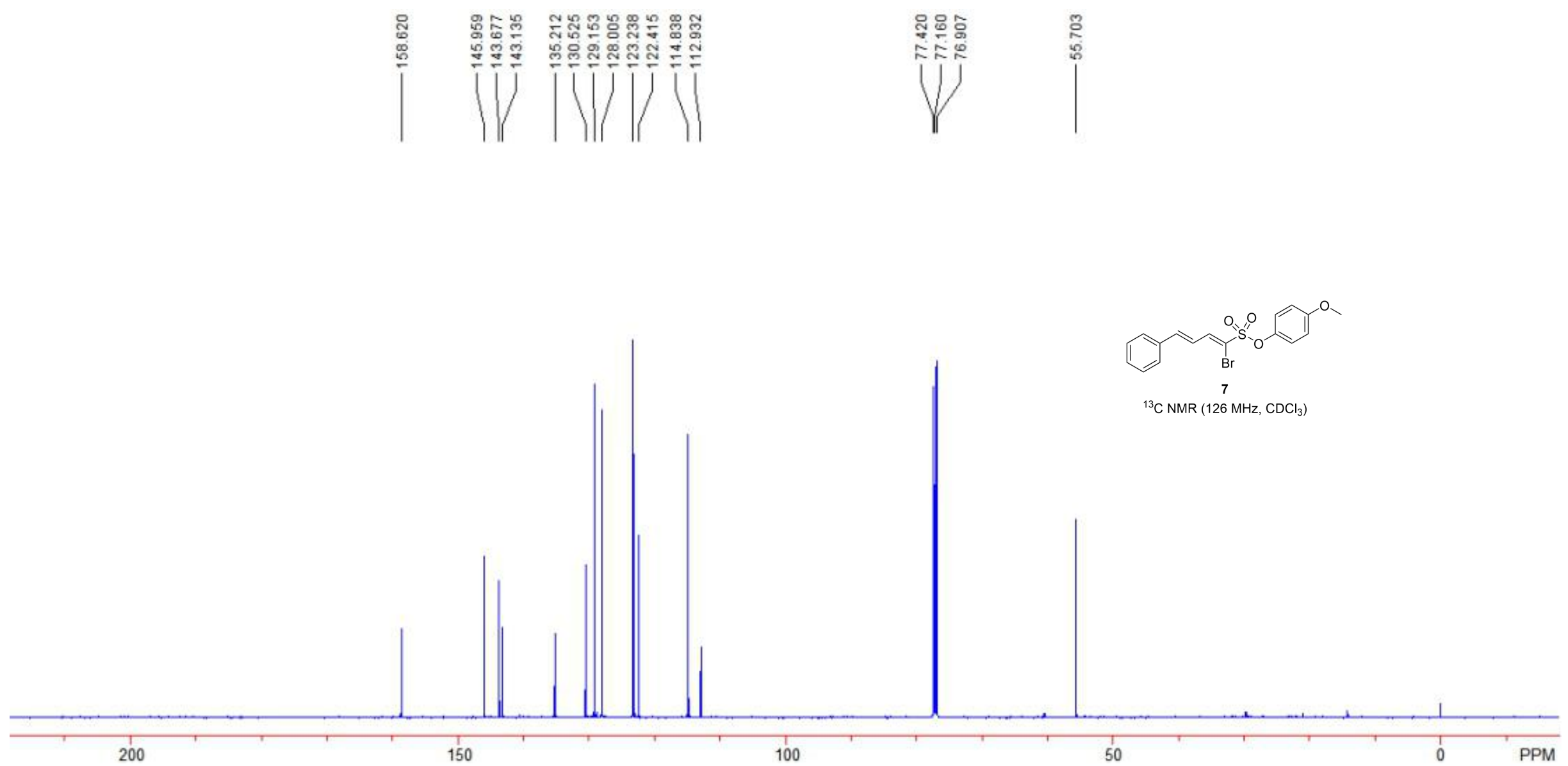

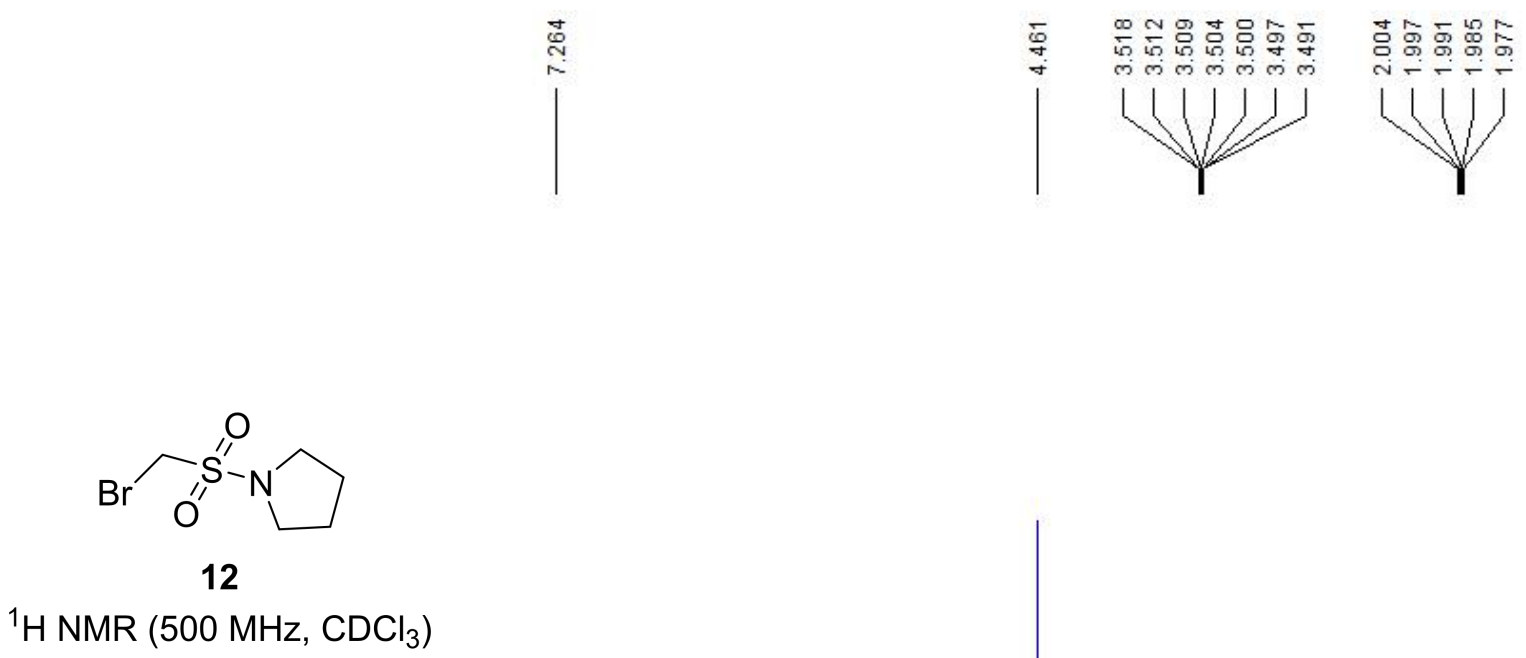

${ }^{1} \mathrm{H}$ NMR $\left(500 \mathrm{MHz}, \mathrm{CDCl}_{3}\right)$ 


\section{Data of Crystal Structure of $3 f a$.}

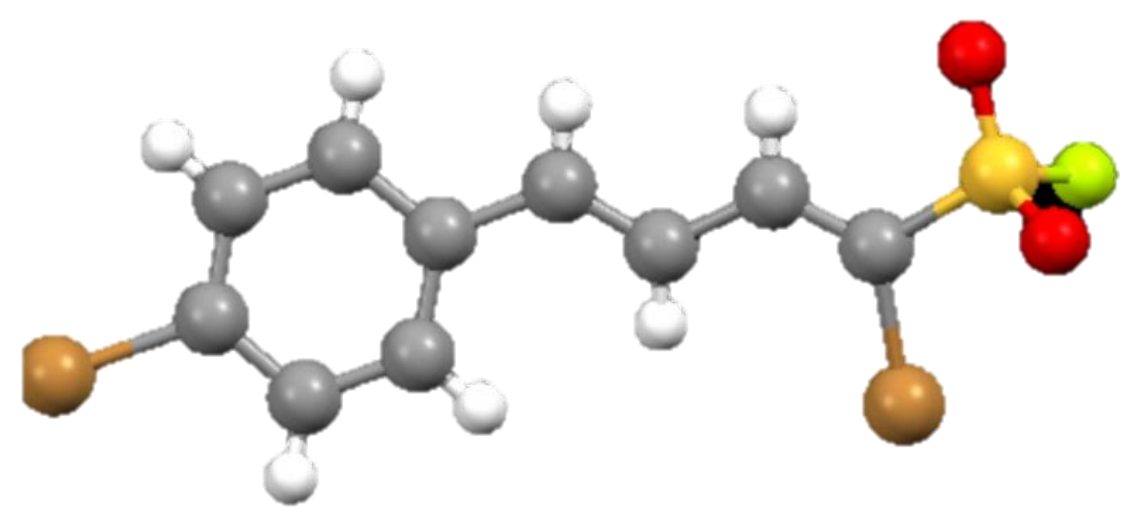

The purified compound $\mathbf{3 f a}$ about $100 \mathrm{mg}$ is dissolved in ethyl acetate and placed in a dark cabinet to slowly evaporate. After several days, a colorless bulk crystal is obtained. The X-ray crystal-structure determinations were obtained on a Bruker Smart-1000 CDCC diffractometer (graphite-monochromated Mo Ka radiation, $\lambda=0.71073 \mathrm{~nm}$ ) at $298(2) \mathrm{K}$. The crystal structure has been deposited at the Cambridge Crystallographic Data Centre (CCDC 1976921).

The ellipsoid contour probability level in the caption is $50 \%$.

Table S6. Crystal data and structure refinement for $190529 \mathrm{c}$.

Identification code

Empirical formula

Formula weight

Temperature

Wavelength

Crystal system, space group

Unit cell dimensions

Volume

Z, Calculated density

Absorption coefficient
$190529 \mathrm{c}$

$\mathrm{C} 10 \mathrm{H} 7 \mathrm{Br} 2 \mathrm{~F}$ O2 S

370.04

298(2) K

$0.71073 \mathrm{~A}$

Monoclinic, P2(1)/n

$$
\begin{gathered}
\mathrm{a}=7.1200(6) \mathrm{A} \quad \text { alpha }=90 \mathrm{deg} . \\
\mathrm{b}=7.8736(6) \text { A } \quad \text { beta }=93.4650(10) \mathrm{deg} . \\
\mathrm{c}=21.6656(19) \text { A } \quad \text { gamma }=90 \mathrm{deg} .
\end{gathered}
$$

$1212.35(17) \mathrm{A}^{\wedge} 3$

4, $2.027 \mathrm{Mg} / \mathrm{m}^{\wedge} 3$

$6.852 \mathrm{~mm}^{\wedge}-1$ 
$\mathrm{F}(000)$

Crystal size

Theta range for data collection

Limiting indices

Reflections collected / unique

Completeness to theta $=25.02$

Absorption correction

Max. and min. transmission

Refinement method

Data / restraints / parameters

Goodness-of-fit on $\mathrm{F}^{\wedge} 2$

Final $\mathrm{R}$ indices [I $>2 \operatorname{sigma}(\mathrm{I})]$

$\mathrm{R}$ indices (all data)

Largest diff. peak and hole
712

$0.30 \times 0.18 \times 0.15 \mathrm{~mm}$

2.75 to $25.02 \mathrm{deg}$.

$-8<=\mathrm{h}<=8,-9<=\mathrm{k}<=7,-22<=\mathrm{l}<=25$

$5699 / 2145[\mathrm{R}($ int $)=0.0246]$

$99.8 \%$

Semi-empirical from equivalents

0.4263 and 0.2330

Full-matrix least-squares on $\mathrm{F}^{\wedge} 2$

$2145 / 0 / 145$

1.090

$\mathrm{R} 1=0.0330, \mathrm{wR} 2=0.0789$

$\mathrm{R} 1=0.0458, \mathrm{wR} 2=0.0825$

0.329 and -0.628 e. $\mathrm{A}^{\wedge}-3$ 